University of Tennessee Health Science Center

UTHSC Digital Commons

\title{
Biodegradable Polymeric Biomaterials in Different Forms for Long-acting Contraception and Drug Delivery to the Eye and Brain
}

\author{
Dileep Reddy Janagam \\ University of Tennessee Health Science Center
}

Follow this and additional works at: https://dc.uthsc.edu/dissertations

Part of the Pharmaceutics and Drug Design Commons

\section{Recommended Citation}

Janagam, Dileep Reddy (http://orcid.org/0000-0002-7235-7709), "Biodegradable Polymeric Biomaterials in Different Forms for Long-acting Contraception and Drug Delivery to the Eye and Brain" (2017). Theses and Dissertations (ETD). Paper 425. http://dx.doi.org/10.21007/etd.cghs.2017.0429.

This Dissertation is brought to you for free and open access by the College of Graduate Health Sciences at UTHSC Digital Commons. It has been accepted for inclusion in Theses and Dissertations (ETD) by an authorized administrator of UTHSC Digital Commons. For more information, please contact jwelch30@uthsc.edu. 


\title{
Biodegradable Polymeric Biomaterials in Different Forms for Long-acting Contraception and Drug Delivery to the Eye and Brain
}

\author{
Abstract \\ Efficacy of many of the new and existing therapeutics is often hampered by the lack of an effective and \\ compliant method of delivery. Typically, drugs have poor water solubility, short half-lives, and low \\ permeability across the biological membranes. The result is low bioavailability of the drugs at the target \\ site and can cause toxicity and side effects at high doses. Often the conventional dosage forms fail to \\ overcome these limitations. In the recent decades, biodegradable polymeric drug delivery systems have \\ emerged as promising candidates to solve the challenges of poor solubility, low permeability and \\ sustained release owing to the advantages of biocompatibility, versatility, and tunable drug release. \\ Polyesters and polysaccharides are the most common polymers that were explored for drug delivery \\ applications because of their unique advantages including non-toxic nature, wide availability, relatively low \\ cost, and flexibility in chemistry. Although a major progress has been in the field of drug delivery, still there \\ are unmet medical needs which require new materials for delivering drugs such as, injectable systems \\ that can achieve long-term contraception (five months or longer) at low cost, and drug delivery systems \\ that can enhance the permeability of drugs across ocular/blood-brain barriers and sustain release as well \\ for treating chronic diseases such as diabetic retinopathy in the eye and Alzheimer's disease in the brain. \\ Therefore, this research has evaluated the potential of different biodegradable polymeric biomaterials \\ based on polyesters or polysaccharides for long-acting contraception and drug delivery to the eye and \\ brain to resolve the issues such as poor compliance and adherence to the existing contraceptive dosage \\ forms or poor solubility and permeability of the drugs across ocular/blood-brain barriers. The first system \\ includes polyester-based injectable in situ forming depot systems (ISD) for long-acting contraception. The \\ aim of this project was to develop injectable ISD system containing levonorgestrel (LNG) for \\ contraceptive effect for five months or longer after single shot that helps to reduce unintended \\ pregnancies with high patient compliance and low cost. A series of LNG-containing ISD formulations \\ were designed by employing unique strategies which include the use of poly(lactic acid-co-glycolic acid), \\ poly(lactic acid) with different biodegradable properties, and blends of these polyesters, use solvent \\ mixtures of N-methyl-2-pyrrolidone, triethyl citrate, benzyl benzoate, and vary the polymer/solvent ratios, \\ and various drug loadings. The formulations were evaluated for viscosity, initial burst, in vitro and in vivo \\ long-term release. In vivo investigation in rats showed the sustained-release pharmacokinetic profile of \\ LNG from the ISD formulations for at least five months and continued for more than seven months \\ depending on the composition, and the vaginal cytology studies have demonstrated that formulations \\ have successfully suppressed the rat estrous cycle. After the end of the treatment, a rapid and predictable \\ return of fertility was observed in rats. The optimized lead formulation has shown promising injectability \\ $(23 \mathrm{G})$ and low initial in vivo burst profiles. The results suggested that the developed LNG-ISD \\ formulations have a great potential for developing into future robust affordable long-acting contraceptive \\ products for improving patient compliance and adherence. Another type of polymeric biomaterial \\ systems that were evaluated in this study includes polysaccharide-based biodegradable nanoparticles for \\ drug delivery across ocular and blood-brain barriers. Depending on the need of the therapeutic \\ application, two types of polysaccharide-based nanoparticles were investigated for their drug delivery \\ feasibility which includes: (a) Poly( $\mathrm{N}$-isopropylacrylamide-co-Dextran-lactateHEMA) nanogels for the \\ potential delivery of hydrophilic peptide (insulin) across ocular barriers for the treatment of diabetic \\ retinopathy. The in vitro, and ex vivo studies showed that the developed nontoxic nanogels have great \\ potential to enhance the drug permeability across ocular barriers including the in vitro retinal pigment \\ epithelium, sclera and cornea barriers for treating diabetic retinopathy; and (b) $\beta$-cyclodextrin-poly $(\beta$ - \\ amino ester) nanoparticles as potential drug carriers to enhance the solubility and blood-brain barrier \\ (BBB) permeability of 17-N-allylamino-17-demethoxygeldanamycin (17-AAG) to treat Alzheimer's disease. \\ The nanoparticles sustained the release of 17-AAG for at least one week in vitro and showed increased
}


permeability (2-fold) of the 17-AAG across BBB in vivo in mice, and resulted in enhanced expression of the Hsp70 protein in the brain. In conclusion, the developed biodegradable polymeric biomaterials have shown potential to be used in long-acting contraception and drug delivery to the eye and brain.

\section{Document Type}

Dissertation

Degree Name

Doctor of Philosophy (PhD)

\section{Program}

Pharmaceutical Sciences

\section{Research Advisor}

Tao L. Lowe, Ph.D.

\section{Keywords}

blood-brain barrier, In situ depot, levonorgestrel, ocular, polyester, polysaccharide

\section{Subject Categories}

Medicine and Health Sciences | Pharmaceutics and Drug Design | Pharmacy and Pharmaceutical

Sciences

\section{Comments}

Two year embargo expires February 2019. 
Biodegradable Polymeric Biomaterials in Different Forms for Long-acting Contraception and Drug Delivery to the Eye and Brain

\author{
A Dissertation \\ Presented for \\ The Graduate Studies Council \\ The University of Tennessee \\ Health Science Center
}

\author{
In Partial Fulfillment \\ Of the Requirements for the Degree \\ Doctor of Philosophy \\ From The University of Tennessee
}

By

Dileep Reddy Janagam

May 2017 
Portions of Chapter 2 (C) 2015 by Springer and Elsevier.

Portions of Chapter 3 (C) 2013 by Springer.

Portions of the Appendix (C) 2015 by Elsevier.

All other material (C) 2017 by Dileep Reddy Janagam.

All rights reserved. 


\section{DEDICATION}

Dedicated to my loving family. 


\section{ACKNOWLEDGEMENTS}

I would like to take this opportunity to express my heartfelt thanks to all those people who have been involved either directly or indirectly, at various stages of my journey at University of Tennessee Health Science Center (UTHSC). I may not be able to list all the names here, but I sincerely appreciate all the unforgettable help, support, advice, and encouragement I have received at UTHSC.

First and foremost, I would like to express my sincerest thanks with a great gratitude to my advisor, Dr. Tao Lowe, for her continuous support, encouragement and guidance throughout the span of my graduate studies. She was always available to discuss my research plans, results, and manuscript; I truly appreciate her time, patience, and understanding. Her guidance helped me in all the time of my research, writing manuscripts, and dissertation, and also helped me to advance in my educational and personal growth. Apart from the academic and research skills, she has constantly encouraged and supported me to take up the leadership roles and improve my skills set in all other aspects that would help me in making a successful career. I am very grateful to her, and it has been an honor to work with her.

I express my deep gratitude to all the graduate committee members Dr. James R. Johnson, Dr. Bernd Meibohm, Dr. James R. Johnson, and Dr. Joel Bumgardner for their time, support, useful suggestions, and insightful comments. I am thankful for the kindness and academic support they have offered me.

I sincerely thank our collaborator Dr. James Johnson for his insight and expertise in injectable in situ depot formulations that helped guide my work as it progressed. I would like to thank Dr. Bernd Meibohm for allowing to use his lab facilities. I thank our collaborator Dr. Timothy Mandrell, and Ms. Stacey L Barnett for their help and support in animal studies. Thanks to Dr. Randy Buddington and his group (University of Memphis), Dr. Charles W. Leffler, Mr. Alexander Fedine, Dr. Dianna for providing the porcine eyeball samples for the permeability studies. I thank Dr. Lichong $\mathrm{Xu}$ for his support with the AFM studies of the nanogles. I thank our collaborator Dr. FrancescaFang Liao, and her group member Dr. Bin Wang for their assistance in in vivo studies with cyclodextrin-based nanoparticles for brain delivery. I also extend my sincere thanks to our collaborator Dr. Anthony Guiseppi-Elie (Texas A\&M University) and his group members (Dr. Kotanen and others) for their assistance related to the poly(HEMA)-based hydrogel characterization project.

My thanks to all my past and present lab members Dr. Linfeng Wu, Dr. Kamaljit Kaur, Dr. Seshulatha Jamalapuram, Dr. Suryatheja Ananthula, Dr. Lizhu Wang, Dr. Xiaoxun Li, Dr. Misra, Keegan Compton, Chandana Damera for their help and support at various stages of the work, and even more, for creating a friendly atmosphere. I am grateful to the assistance received from various lab personnel outside my research groups from our department. 
I would like to thank all the faculty members of the Department of Pharmaceutical Sciences for their invaluable academic support. I thank our staff members Brenda Thornton, Corliss Finlay, Shelley Cannioto, Caroll Taylor, Lisa Schaffer, and Mary Newell for their support. My gratitude also extends to University of Tennessee Health Science Center, College of Pharmacy, and College of Graduate Health Sciences for the facilities, equipment, and financial support. I thank all the funding agencies that supported my research.

Many thanks to all my friends at UT and in Memphis who has made my graduate study a memorable one. They all have been a valuable source of friendship and advice over the past years.

Last but not the least, I am very much grateful to my wife Sri Chandana Damera, my mom, my dad and my brother, this is the culmination of all of their love and effort, and without them I could not have made it this far. Finally, I need to thank God for giving all these opportunities to learn new things and meet great people. 


\begin{abstract}
Efficacy of many of the new and existing therapeutics is often hampered by the lack of an effective and compliant method of delivery. Typically, drugs have poor water solubility, short half-lives, and low permeability across the biological membranes. The result is low bioavailability of the drugs at the target site and can cause toxicity and side effects at high doses. Often the conventional dosage forms fail to overcome these limitations. In the recent decades, biodegradable polymeric drug delivery systems have emerged as promising candidates to solve the challenges of poor solubility, low permeability and sustained release owing to the advantages of biocompatibility, versatility, and tunable drug release. Polyesters and polysaccharides are the most common polymers that were explored for drug delivery applications because of their unique advantages including non-toxic nature, wide availability, relatively low cost, and flexibility in chemistry. Although a major progress has been in the field of drug delivery, still there are unmet medical needs which require new materials for delivering drugs such as, injectable systems that can achieve long-term contraception (five months or longer) at low cost, and drug delivery systems that can enhance the permeability of drugs across ocular/blood-brain barriers and sustain release as well for treating chronic diseases such as diabetic retinopathy in the eye and Alzheimer's disease in the brain. Therefore, this research has evaluated the potential of different biodegradable polymeric biomaterials based on polyesters or polysaccharides for long-acting contraception and drug delivery to the eye and brain to resolve the issues such as poor compliance and adherence to the existing contraceptive dosage forms or poor solubility and permeability of the drugs across ocular/blood-brain barriers.
\end{abstract}

The first system includes polyester-based injectable in situ forming depot systems (ISD) for long-acting contraception. The aim of this project was to develop injectable ISD system containing levonorgestrel (LNG) for contraceptive effect for five months or longer after single shot that helps to reduce unintended pregnancies with high patient compliance and low cost. A series of LNG-containing ISD formulations were designed by employing unique strategies which include the use of poly(lactic acid-co-glycolic acid), poly(lactic acid) with different biodegradable properties, and blends of these polyesters, use solvent mixtures of N-methyl-2-pyrrolidone, triethyl citrate, benzyl benzoate, and vary the polymer/solvent ratios, and various drug loadings. The formulations were evaluated for viscosity, initial burst, in vitro and in vivo long-term release. In vivo investigation in rats showed the sustained-release pharmacokinetic profile of LNG from the ISD formulations for at least five months and continued for more than seven months depending on the composition, and the vaginal cytology studies have demonstrated that formulations have successfully suppressed the rat estrous cycle. After the end of the treatment, a rapid and predictable return of fertility was observed in rats. The optimized lead formulation has shown promising injectability (23 G) and low initial in vivo burst profiles. The results suggested that the developed LNG-ISD formulations have a great potential for developing into future robust affordable long-acting contraceptive products for improving patient compliance and adherence. 
Another type of polymeric biomaterial systems that were evaluated in this study includes polysaccharide-based biodegradable nanoparticles for drug delivery across ocular and blood-brain barriers. Depending on the need of the therapeutic application, two types of polysaccharide-based nanoparticles were investigated for their drug delivery feasibility which includes: (a) Poly(N-isopropylacrylamide-co-Dextran-lactateHEMA) nanogels for the potential delivery of hydrophilic peptide (insulin) across ocular barriers for the treatment of diabetic retinopathy. The in vitro, and ex vivo studies showed that the developed nontoxic nanogels have great potential to enhance the drug permeability across ocular barriers including the in vitro retinal pigment epithelium, sclera and cornea barriers for treating diabetic retinopathy; and (b) $\beta$-cyclodextrin-poly ( $\beta$-amino ester) nanoparticles as potential drug carriers to enhance the solubility and blood-brain barrier (BBB) permeability of 17-N-allylamino-17-demethoxygeldanamycin (17-AAG) to treat Alzheimer's disease. The nanoparticles sustained the release of 17-AAG for at least one week in vitro and showed increased permeability (2-fold) of the 17-AAG across BBB in vivo in mice, and resulted in enhanced expression of the Hsp 70 protein in the brain.

In conclusion, the developed biodegradable polymeric biomaterials have shown potential to be used in long-acting contraception and drug delivery to the eye and brain. 


\section{TABLE OF CONTENTS}

\section{CHAPTER 1. BIODEGRADABLE POLYMERIC BIOMATERIALS FOR

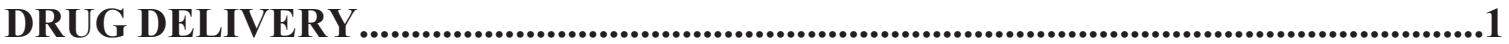





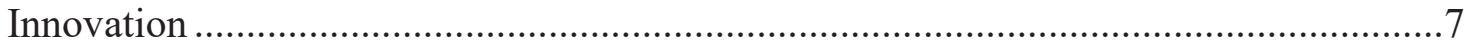

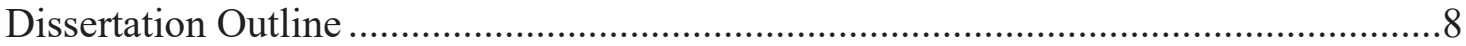

\section{CHAPTER 2. INJECTABLE IN SITU FORMING DEPOT SYSTEMS FOR} LONG-ACTING CONTRACEPTION ...................................................................10

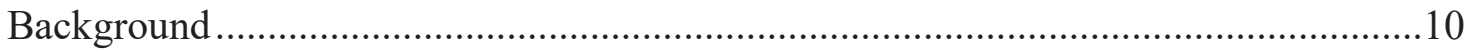

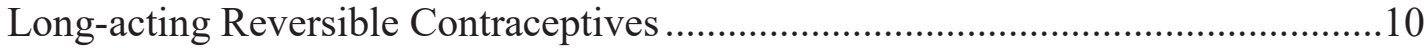

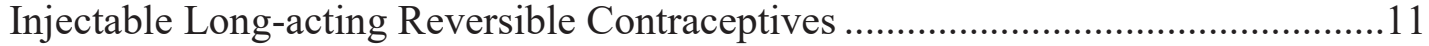

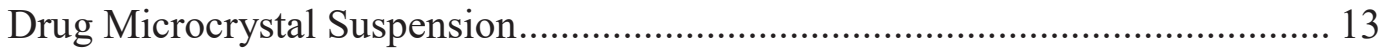

Polymer-based Drug Delivery Systems .......................................................... 13

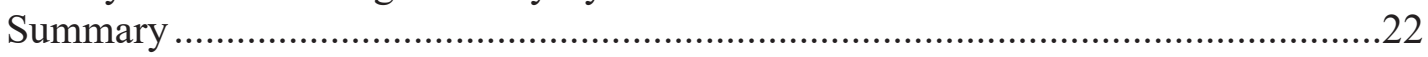

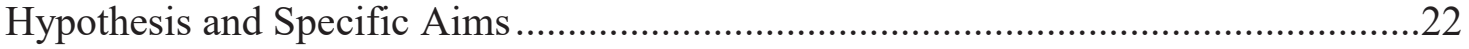

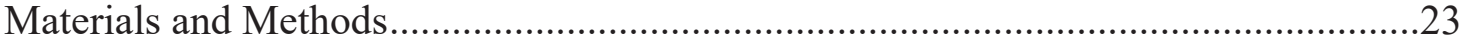

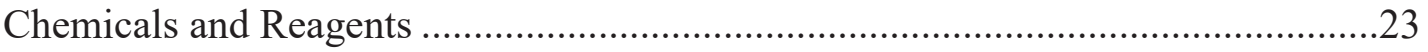

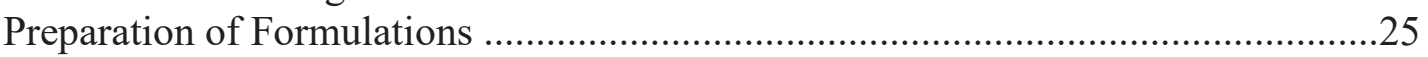

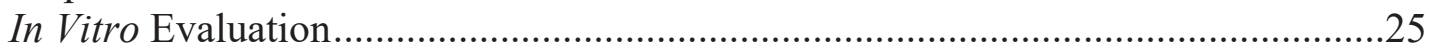

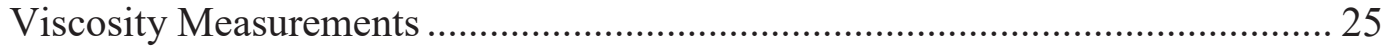

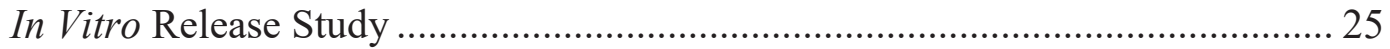

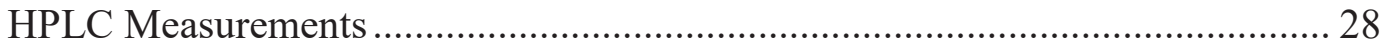

In Vivo Evaluation.............................................................................................28

LC-MS/MS Method Development and Validation for Analysis of LNG in Rat

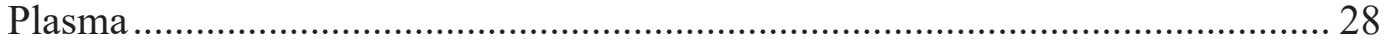

In Vivo Pharmacokinetic Study of Formulations ................................................. 33

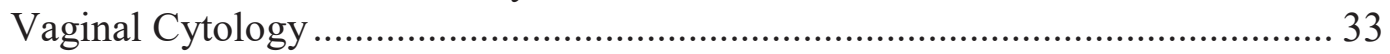



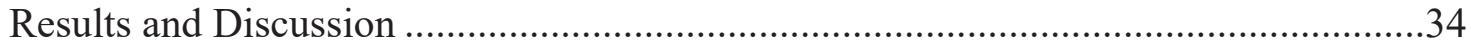

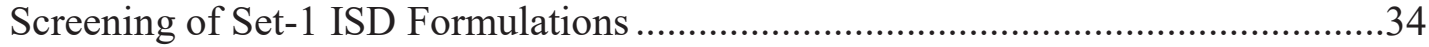

In Vitro Release of ISD Formulations Set-1 .................................................... 36

LC-MS/MS Method Development and Validation for Analysis of LNG in Rat

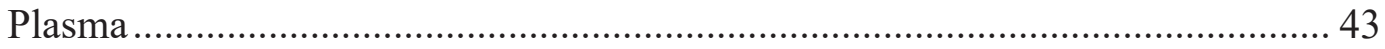

In Vivo Evaluation of the Selected ISD Formulations $(55,64$ and 96)............... 55

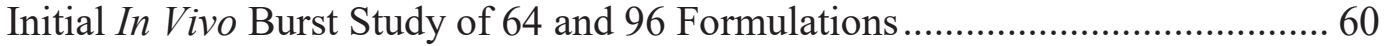

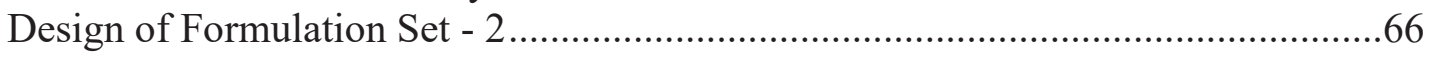

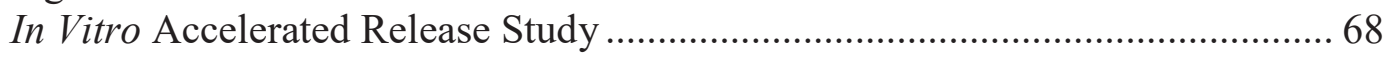





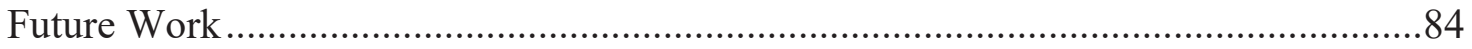




\section{CHAPTER 3. POLYSACCHARIDE-BASED NANOPARTICLES FOR DRUG DELIVERY ACROSS OCULAR/BLOOD-BRAIN BARRIERS ............................85}

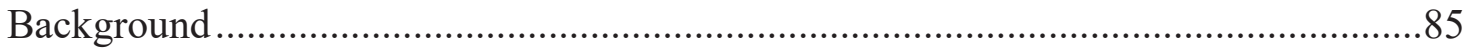

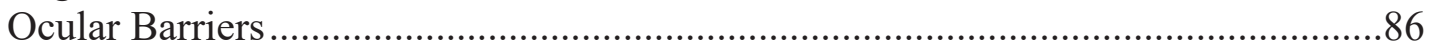

Routes of Administration and Ocular Barriers ............................................. 86

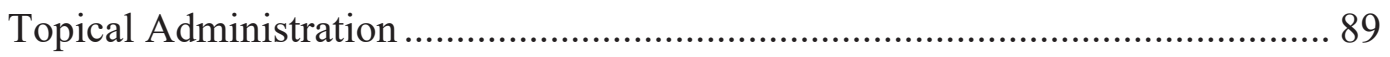

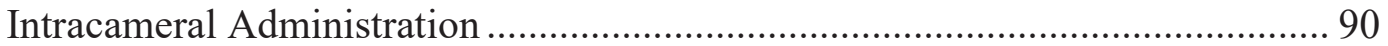

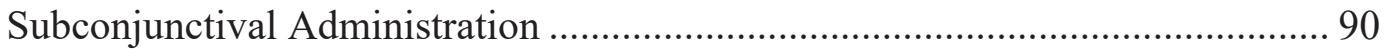

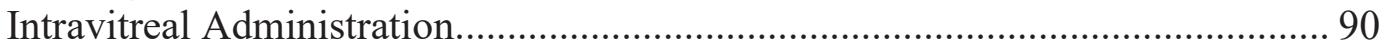

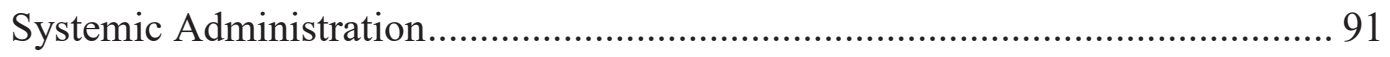

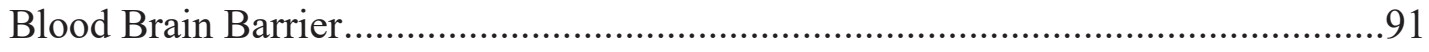

Strategies for Circumventing the BBB ........................................................ 93

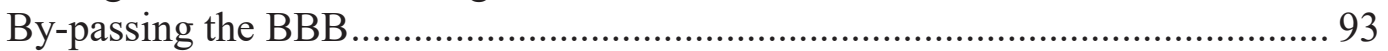

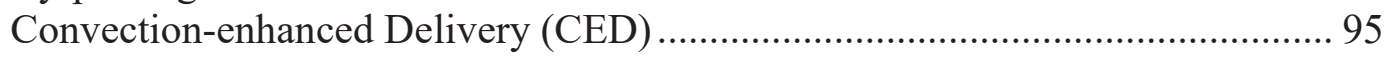

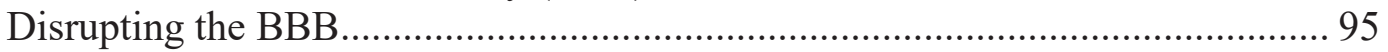





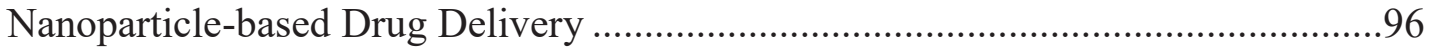

Polysaccharide-based Nanoparticles for Drug Delivery to the Eye and Brain ..........98



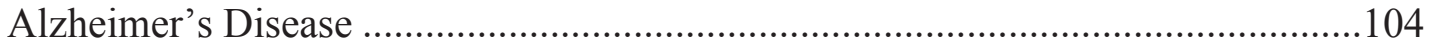

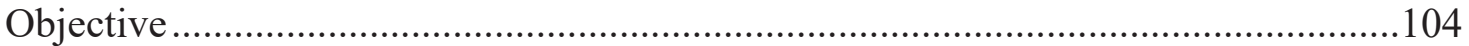

Biodegradable Poly(NIPAAm-co-DEXlactateHEMA) Nanogels for Ocular

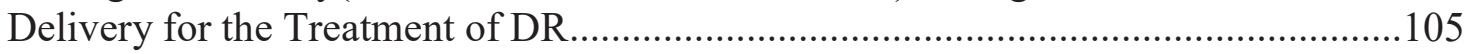

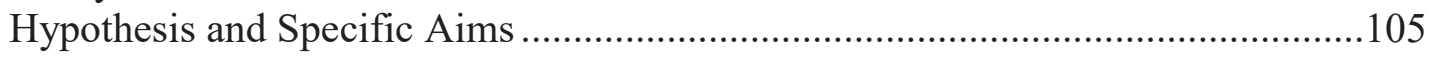

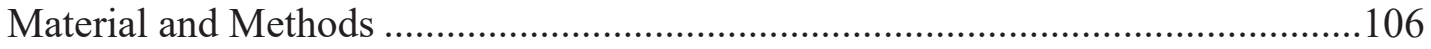



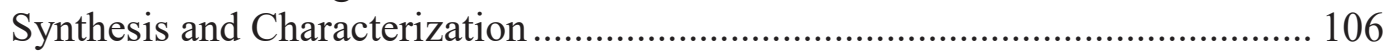

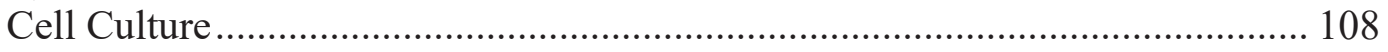

In Vitro Cytotoxicity of the Nanogels.......................................................... 108

In Vitro Permeability across ARPE-19 Monolayers ...................................... 110

AFM Measurements.................................................................................. 112

Ex Vivo Permeability across Porcine Ocular Tissues ..................................... 112

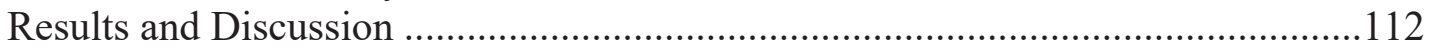

Synthesis and Characterization of Nanogels............................................... 112

In Vitro Permeability of Nanogels ................................................................. 114

Ex Vivo Permeability of Nanogels across Porcine Ocular Tissues .................... 117

Permeability of Porcine Ocular Tissues at Different Stages of Development.... 117

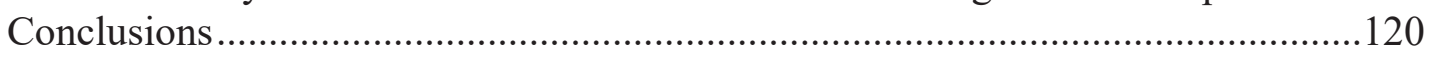

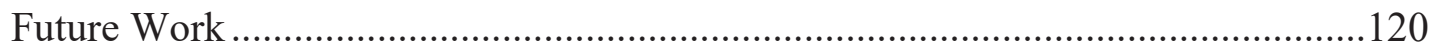

Beta-cyclodextrin-poly( $\beta$-amino ester) (CD-p-AE) Nanoparticles as Drug Carriers

to Enhance BBB Permeability of 17-AAG to Treat AD .........................................123



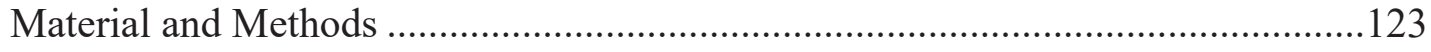

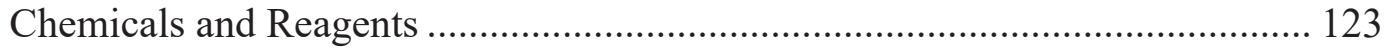

Preparation of 17-AAG-loaded CD-p-AE Nanoparticles ................................. 124 


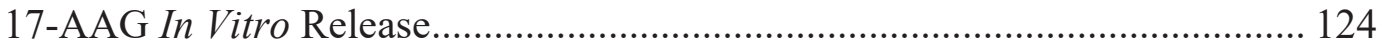

In Vivo Studies of 17-AAG-loaded CD-p-AE Nanoparticles .............................. 124

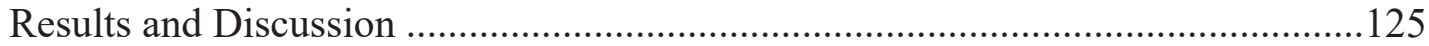

17-AAG Loading and Release from CD-p-AE Nanoparticles ............................ 125

In Vivo Studies of 17-AAG-loaded CD-p-AE Nanoparticles ............................... 127

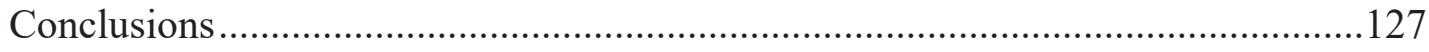

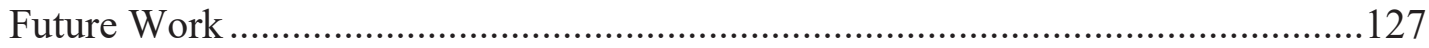

CHAPTER 4. CONCLUSIONS.................................................................................131

LIST OF REFERENCES .......................................................................................133



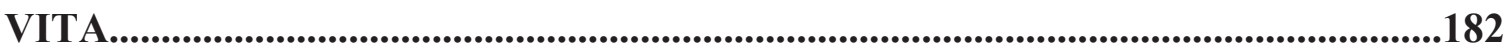




\section{LIST OF TABLES}

Table 1-1. List of polymeric biomaterials for drug delivery..........................................

Table 2-1. Common solvents (plasticizers) and their key features. ................................20

Table 2-2. The composition of set-1 ISD formulations.................................................26

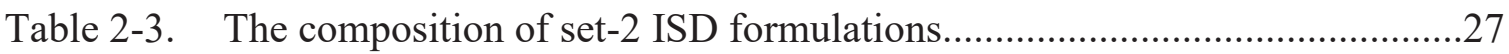

Table 2-4. Release testing conditions used for accelerated in vitro release method development. .........................................................................................29

Table 2-5. The composition and viscosity of the 55, 64 and 96 formulations...............39

Table 2-6. Fitting parameters determined by the linear regression of $\log \left(M_{t} / M_{\infty}\right)$ against $\log t$ in Power law for the LNG release from the depots made of 55, 64 and 96 formulations.

Table 2-7. Linearity and summary of calibration curves for LNG in rat plasma $(n=6)$ with back-calculated concentrations.

Table 2-8. Intra-day assay accuracy and precision determination of LNG standards in rat plasma for 3 consecutive days $(\mathrm{n}=6)$.

Table 2-9. Inter-day assay accuracy and precision determination of LNG standards in rat plasma $(\mathrm{n}=18)$.

Table 2-10. Accuracy and precision for LNG QC samples in rat plasma.

Table 2-11. Matrix effect and recovery efficiency results of LNG QC samples and LNG-D 6 .

Table 2-12. Stability of LNG QC samples at the various experimental condition.

Table 2-13. Pharmacokinetic parameters of $L N G$ in rat plasma $(n=6)$ after SubQ injection of LNG in NMP solution $\left(16 \mathrm{mg} \cdot \mathrm{kg}^{-1}\right)$.

Table 2-14. Pharmacokinetic parameters of LNG solution in NMP, 64 and 96 formulations in rat plasma after SubQ injection.

Table 2-15. Fitting parameters determined by the linear regression of $\log \left(M_{t} / M_{\infty}\right)$ against $\log t$ in Equation 2-5 for the LNG release from the depots made of formulations 96 and 64 under real time and four accelerated release conditions.

Table 2-16. Pharmacokinetic parameters of 96r and 96zz formulations in rat plasma after 24 hours post SubQ injection. 
Table 3-1. Administration routes for delivering drugs to the anterior segment of the eye.

Table 3-2. Chemical structures of various polysaccharides that were used for drug delivery.

Table 3-3. Composition, particle size and zeta potential of the nanogels.

Table A-1. Monomer cocktail formulations for the preparation of varying mol\% TEGDA crosslinked poly(HEMA)-based hydrogels prepared in triplicate. 166

Table A-2. The glass transition temperatures $\left(T_{g}\right)$ from the second heat cycle for the hydrogels with and without $5 \mathrm{~mol} \%$ DMAEMA under different drying processes

Table A-3. The gravimetrically determined degree of hydration ( $\mathrm{DoH} \%)$ of the $\mathrm{p}$ (HEMA)-based hydrogels with and without DMAEMA.

Table A-4. Distribution of water content in the hydrogels possessing 0 or $5 \mathrm{~mol} \%$ DMAEMA and equilibrated in DI water.

Table A-5. Distribution of water content of hydrogel possessing 0 or $5 \mathrm{~mol} \%$ DMAEMA or AEMA and equilibrated in DI water. 


\section{LIST OF FIGURES}

Figure 1-1. The classification and examples of biodegradable polymers used in the fabrication of biomaterials for drug delivery.

Figure 2-1. Flow chart of the advantages, disadvantages, and commercial availability of injectable LARCs.

Figure 2-2. Ring opening polymerization of selected cyclic lactones to give the corresponding polyester polymers.

Figure 2-3. Design and developmental plan of injectable ISD dosage forms. ................24

Figure 2-4. Teflon mold holding the depot for in vitro LNG release studies. 37

Figure 2-5. In vitro cumulative LNG release percentage from set-1 ISD formulations.

Figure 2-6. In vitro cumulative LNG release (A), and in vitro LNG release amount per day (B) from the ISD formulations.

Figure 2-7. In vitro depot pictures of ISD formulations 55, 64 and 96 in Teflon molds after two months release study.

Figure 2-8. The product ion mass spectrum of the $\mathrm{LNG}$ at $\mathrm{m} / \mathrm{z} 313.10$.

Figure 2-9. Representative chromatograms of LNG and internal standard (LNG-D 6 ) ...47

Figure 2-10. Representative calibration curve for LNG in rat plasma.

Figure 2-11. LNG concentration in rat plasma $(\mathrm{n}=6)$ as a function of time after SubQ injection of LNG solution in NMP $\left(16 \mathrm{mg} \cdot \mathrm{kg}^{-1}\right)$.

Figure 2-12. Plasma concentration of LNG (long-term levels) as a function of time after SubQ injections of ISD formulations...

Figure 2-13. Changes in body weights of rats subcutaneously injected with ISD

formulations.

Figure 2-14. Four stages of rat estrous cycle and characteristic features.

Figure 2-15. Representative image of the cycle progression in rats injected with formulation (F-96).

Figure 2-16. Plasma concentration of LNG (initial burst levels) in female SD rats as a function of time over $24 \mathrm{~h}$ after SubQ injection of ISD formulations and control LNG. 
Figure 2-17. Key results summary of 55, 64 and 96 ISD formulations.

Figure 2-18. Short-term (accelerated) in vitro LNG release from formulations 96 (I) and 64 (II) at four accelerated conditions.

Figure 2-19. Power law kinetics (plot of log percent release vs. log of time in days) of formulations.

Figure 2-20. Correlation of accelerated (short-term) release at condition D with realtime (long-term) release testing of formulations 96 (I; triangle) and 64(II; circle).

Figure 2-21. Shear viscosity (A) and LNG in vitro release in accelerated condition D (B) of different ISD formulations. .76

Figure 2-22. Shear viscosities of 64, 96, 96p, 96q, and 96r formulations. .78

Figure 2-23. Shear viscosity (A) and LNG in vitro release in accelerated condition D (B) of the formulations $64,96,96 \mathrm{r}$ and $96 \mathrm{j}$....

Figure 2-24. LNG Plasma concentration measured in rats as a function of time after SubQ injection of $64,96 \mathrm{r}, 96 \mathrm{zz}$ formulations.

Figure 3-1. The structure of the anterior segment of the eye and ocular barriers for drug delivery.

Figure 3-2. Anatomy of the blood-brain barrier (BBB).

Figure 3-3. Intrathecal administration (A); Convection-enhanced delivery (B).

Figure 3-4. Nanocarriers for brain drug delivery.

Figure 3-5. The structure of dextran macromer (DEX-LacateHEMA). 107

Figure 3-6. The synthesis scheme of poly(NIPAAm-co-DEXlactateHEMA) nanogels.

Figure 3-7. Experimental set-up for in vitro permeability.

Figure 3-8. Experimental set-up for ex vivo permeability.

Figure 3-9. Cell viability of the nanogels to hfRPE cells, to nanogels and degradants. 116

Figure 3-10. In vitro transport (A) and permeability (B) of nanogels (63A, 72A) and $70 \mathrm{kDa}$-dextran across ARPE-19 cell monolayer; AFM image of the nanoparticle from the receptor chamber $(\mathrm{C})$.

Figure 3-11. Comparison of nanogels permeability across ex vivo piglet sclera (A) and cornea $(\mathrm{B})$. 
Figure 3-12. Comparison of the thickness of porcine sclera (A) and cornea (B) at different stages of development.

Figure 3-13. Comparison of ex vivo permeability of porcine sclera (A) and cornea (B) at different stages of development.

Figure 3-14. In vitro release of 17-AAG from CD-p-AE nanoparticles...........................126

Figure 3-15. 17-AAG levels in the brain at $24 \mathrm{~h}$ following intravenous injection of 17-AAG alone/17-AAG loaded CD-p-AE nanoparticles.

Figure 3-16. Hsp70 levels in the brain at $24 \mathrm{~h}$ following intravenous injection of 17 AAG alone/17-AAG-loaded CD-p-AE nanoparticles

Figure A-1. Schematic illustration of the polymerized hydrogels

Figure A-2. Shear storage modulus $\left(\mathrm{G}^{\prime}\right)$ and shear loss modulus $\left(\mathrm{G}^{\prime \prime}\right)$ as a function of strain $\%$ (A) and frequency (B) for the hydrogels.

Figure A-3. Stress-strain curve for hydrogels under compression (A); Compression modulus of hydrogels (B); Scheme representing the hydrogen bonding ability of DMAEMA (C).

Figure A-4. Endothermic curves of swollen hydrogels synthesized with and without 5 mol\% DMAEMA.

Figure A-5. Scheme representing the possible hydrogen bonding between AEMA units with adjacent HMMA or HEMA units. 


\section{LIST OF ABBREVIATIONS}

\begin{tabular}{|c|c|}
\hline AD & Alzheimer's disease \\
\hline AEMA & Amino ethyl methacrylate \\
\hline AFM & Atomic force microscopy \\
\hline AMD & Age-related macular degeneration \\
\hline AMT & Adsorptive-mediated transcytosis \\
\hline API & Active pharmaceutical ingredient \\
\hline ARPE-19 & Adult retinal pigmented epithelium cells 19 \\
\hline ATEC & Acetyl triethyl citrate \\
\hline AUC & Area under curve \\
\hline$A \beta$ & $\beta$-amyloid \\
\hline BA & Benzyl Alcohol \\
\hline BAB & Blood-aqueous barrier \\
\hline BB & Benzyl benzoate \\
\hline BBB & Blood-brain barrier \\
\hline BRB & Blood-retinal barrier \\
\hline CDI & N,N'-carbonyl-diimidazole \\
\hline CD-p-AE & $\beta$-Cyclodextrin-poly $(\beta$-amino ester $)$ \\
\hline CED & Convection-enhanced delivery \\
\hline $\mathrm{CL} / \mathrm{F}$ & Apparent total body clearance \\
\hline $\mathrm{C}_{\max }$ & Maximum concentration \\
\hline CSF & Cerebrospinal fluid \\
\hline $\mathrm{CV}$ & Coefficient of variation \\
\hline $\mathrm{Da}$ & Dalton \\
\hline DI $\mathrm{H}_{2} \mathrm{O}$ & Deionized water \\
\hline DI water & Deionized water \\
\hline DI & Deionized \\
\hline DLS & Dynamic light scattering \\
\hline DMA & Dynamic mechanical analyzer \\
\hline DMAEM & Dimethyl amino ethyl methacrylate \\
\hline DMAP & 4-(N,N-diethylamino) pyridine \\
\hline DMEM & Dulbecco's modified eagle medium \\
\hline DMF & $\mathrm{N}, \mathrm{N}$-dimethylformamide \\
\hline DMSO & Dimethyl sulfoxide \\
\hline DMSO & Dimethyl sulfoxide \\
\hline DoH & Degree of Hydration \\
\hline DP & Degree of polymerization \\
\hline DS & Degree of substitution \\
\hline DSC & Differential scanning calorimeter \\
\hline DTAF & 5-(4,6-dichlorotriazinyl) aminofluorescein \\
\hline EA & Ethyl acetate \\
\hline FBS & Fetal bovine serum \\
\hline FITC & Fuorescein isothiocyanate \\
\hline $\mathrm{G}^{\prime \prime}$ & Loss modulus \\
\hline
\end{tabular}




\begin{tabular}{|c|c|}
\hline $\mathrm{G}^{\prime}$ & Storage modulus \\
\hline GA & Glycolic Acid \\
\hline GPC & Gel permeation chromatography \\
\hline HEMA & 2-hydroyethyl methacrylate \\
\hline HEPES & 4-(2-hydroxyethyl)-1-piperazineethanesulfonic acid) \\
\hline hfRPE & Human fetal retinal pigment epithelial cells \\
\hline HPLC & High-performance liquid chromatography \\
\hline Hsp & Heat shock protein \\
\hline IACUC & Institutional Animal Care and Use Committee \\
\hline iHsp & Inhibitor of heat shock protein \\
\hline ISD & In situ forming depot/implant \\
\hline IUD & Intrauterine device \\
\hline IUS & Intrauterine system \\
\hline iv & Inherent viscosity \\
\hline $\mathrm{kDa}$ & Kilo Dalton \\
\hline $\mathrm{kPa}$ & Kilo Pascal \\
\hline LA & Lactic Acid \\
\hline LARCs & Long-acting reversible contraceptives \\
\hline LCST & Lower critical solution temperature \\
\hline LLOQ & Lower limit of quantitation \\
\hline LNG & Levonorgestrel \\
\hline LNG-B & Levonorgestrel butanoate \\
\hline LOD & Limit of detection \\
\hline LOQ & Limit of quantitation \\
\hline LVE & Linear viscoelastic \\
\hline MCWO & Molecular weight cut off \\
\hline MTT & 3-(4,5-dimethylthiazol-2-yl)-2,5-diphenyltetrazolium bromide \\
\hline MW & Molecular weight \\
\hline NET & Norethisterone \\
\hline NIPAAm & $\mathrm{N}$-isopropyl acrylamide \\
\hline NMP & N-Methyl-2-pyrrolidone \\
\hline OTS & Octadecyltrichlorosilane \\
\hline PBS & Phosphate buffer saline \\
\hline PBS & Phosphate buffer saline \\
\hline PCL & Poly( $\varepsilon$-caprolactone) \\
\hline PLA & Poly(lactide) \\
\hline PLGA & Poly(lactide-co-glycolide) \\
\hline Poly(HEMA) & Poly(2-hydroxyethyl methacrylate) \\
\hline PTFE & Polytetrafluoroethylene \\
\hline $\mathrm{QC}$ & Quality control \\
\hline RE & Recovery efficiency \\
\hline RP-HPLC & Reverse phase high-performance liquid chromatography \\
\hline $\mathrm{S} / \mathrm{N}$ & Signal to noise ratio \\
\hline SD & Sprague-Dawley \\
\hline SD & Standard deviation \\
\hline SE & Standard error \\
\hline
\end{tabular}




$\begin{array}{ll}\text { SubQ } & \text { Subcutaneous } \\ \text { TEC } & \text { Triethyl citrate } \\ \text { TEER } & \text { Trans-epithelial electrical resistance } \\ \text { TEGDA } & \text { Tetra(ethylene glycol) diacrylate } \\ \mathrm{T}_{\mathrm{g}} & \text { Glass transition temperature }( \\ \mathrm{THF} & \text { Tetrahydrofuran } \\ \mathrm{T}_{\max } & \text { Time at maximum concentration } \\ \mathrm{UPLC} & \text { Ultra Performance Liquid Chromatography } \\ \mathrm{V}_{\mathrm{d}} / \mathrm{F} & \text { Apparent volume of distribution } \\ \mathrm{V}_{\mathrm{s}} & \text { Versus } \\ \mathrm{W}_{\mathrm{fb}} & \text { Freezable bound water } \\ \mathrm{W}_{\mathrm{ff}} & \text { Freezable free water } \\ \mathrm{W}_{\mathrm{nfb}} & \text { Non-freezable bound water } \\ \mathrm{Wt}_{\mathrm{t}} & \text { Weight percentage } \\ 17-\mathrm{AAG} & \text { 17-Allylamino-17-demethoxygeldanamycin }\end{array}$




\section{CHAPTER 1. BIODEGRADABLE POLYMERIC BIOMATERIALS FOR DRUG DELIVERY}

\section{Introduction}

New drug discovery and development is a multi-stage complex and laborintensive process requiring years of time and large amounts of money. According to the latest report by the Tufts Center for the Study of Drug Development, the average expenses involved in the new drug development from the discovery stage to market approval was estimated to be $\$ 2.558$ billion USD [1,2]. Consequently, enhancing the safety and efficacy of current therapeutics has been attempted using various strategies such as novel drug delivery systems, personalized medication, dose alteration, and therapeutic drug monitoring. Delivering drugs at a sustained and controlled rate, targeted delivery using drug delivery systems was the most attractive method that has been pursued extensively [3]. New delivery systems can be developed at relatively low cost and time than new drug discovery, and this allows to maximize the return on investment, giving a second life to existing therapeutics, with improved efficacy and patient compliance [4]. Further, in the recent years, the concept of the drug has changed from the traditional small molecules to the biologics (such as peptides, proteins, monoclonal antibodies, RNA, DNA), and this change required the exploration of new delivery systems because of the failure of the conventional systems. Moreover, with the progressive shrinkage of the pipelines from pharmaceutical organizations and the release of many of the drugs from the patent, drug delivery systems are expected to play an increasingly critical part in the present pharmaceutical landscape [5]. The market related to drug delivery technology was projected to reach $\$ 1,504.7$ billion USD by 2020 from $\$$ $1,048.1$ billion in 2015 [6].

"Drug delivery describes the method and approach to delivering drugs or pharmaceuticals and other xenobiotics to their site of action within an organism, with the goal of achieving a therapeutic outcome"[1]. Currently, it is widely acknowledged that the success of a therapeutic molecule depends strongly on the function and efficiency of the drug delivery system. One of the strategies toward this goal has been the use of biodegradable polymeric biomaterials for drug delivery [7]. Numerous literature reports support that polymers have been used as a primary tool to control the drug release rate from the formulations [7-10]. Biodegradable polymers represent an emerging field, and significant advances have been made with regard to the development of biodegradable polymeric materials for formulation and drug delivery applications because of several advantages such as biodegradability, biocompatibility, versatility, and tunable drug release [10]. Each drug delivery application is unique and demands materials with specific physiochemical, biological, mechanical and degradation properties to meet the requirements to provide effective therapy. As a result, a wide range of natural or synthetic biodegradable polymers which can undergo hydrolytic or enzymatic degradation are being investigated. The classification and examples of synthetic and natural biodegradable polymers that have been widely used as biodegradable polymeric biomaterials for drug delivery are given in Figure 1-1. These polymers undergo 


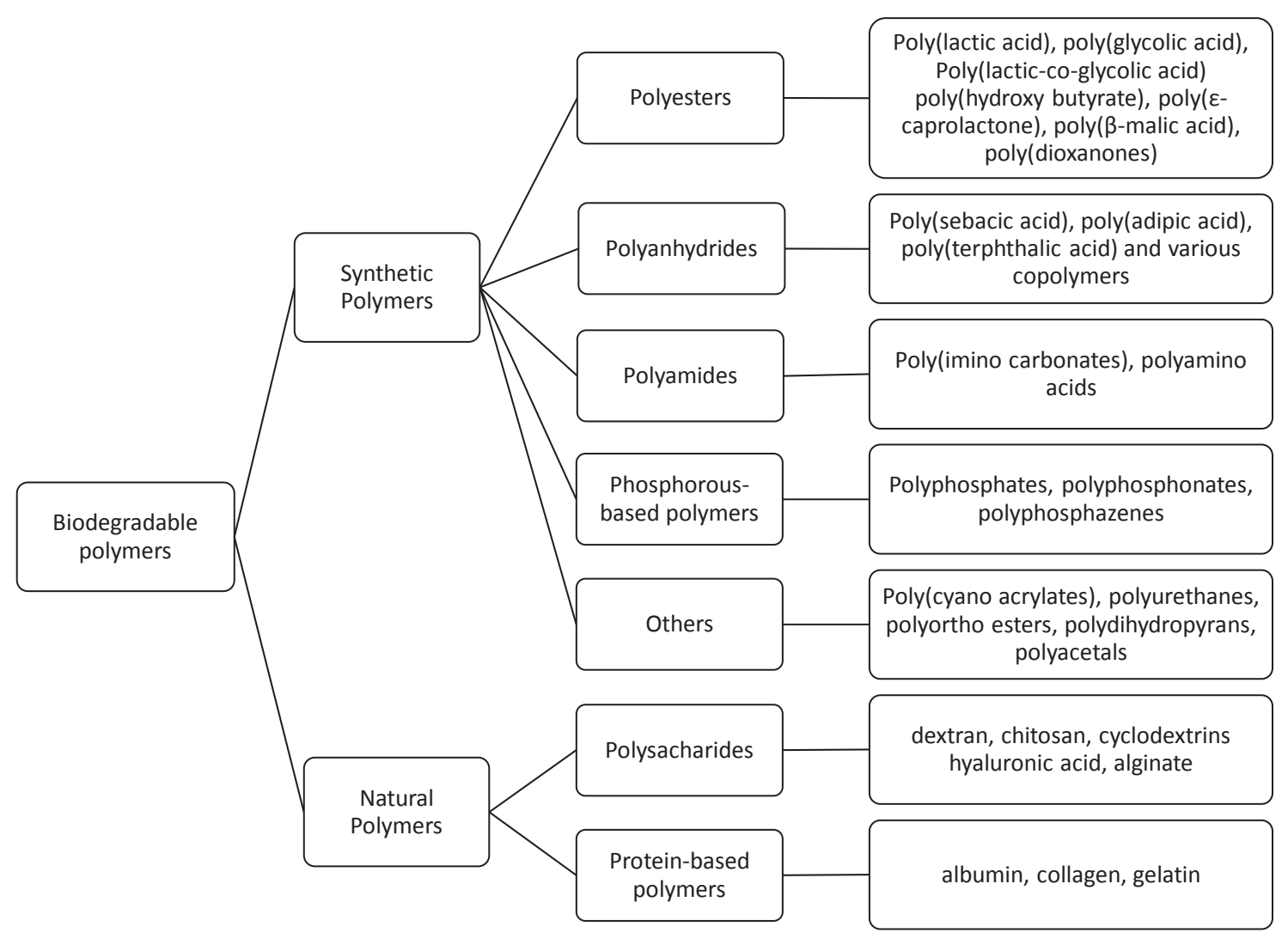

Figure 1-1. The classification and examples of biodegradable polymers used in the fabrication of biomaterials for drug delivery. 
biodegradation through cleavage of hydrolytically or enzymatically sensitive bonds in the polymer chain leading to polymer erosion [11]. Among the various polymers, polyesters and polysaccharides are the extensively investigated polymers for drug delivery applications [12-22].

A significant emphasis was made on innovation for improving the delivery of therapeutics by designing various drug delivery systems with various objectives which include: i) treat different conditions located at different physiological locations; ii) sustained drug release for reduction in dosage frequency and better patient compliance; iii) minimize the drug concentration fluctuations in vivo and maintain constant drug levels within therapeutic window; iv) localized drug delivery; v) enhanced delivery of poorly water-soluble drugs; vi) targeted delivery of therapeutics to a cell- or tissue in a specific manner; vii) transport of drugs across tight epithelial and endothelial barriers (transcytosis); viii) delivery of large-molecule therapeutics to intracellular sites of action; ix) co-delivery of multiple drugs or therapeutic modality for combination therapy; $\mathrm{x}$ ) visualization or diagnosis of diseased sites by drug delivery through combining therapeutic agents with imaging modalities (theranostics) for real-time monitoring on the in vivo efficacy of a drug; xi) reduced incidence of toxicity and side effects; xii) reducing drug clearance in the path, and improving drug stability and bioavailability at the targets [6-10, 23-27]. The other objective or advantage is that manufacturer's development of a new delivery system of a product helps in extending the patent life [28].

Depending on the drug, application, and therapeutic route, each approach will be unique and requires polymeric biomaterials with different physical, chemical and biological properties. As a result, we see drug systems that come in all shapes and sizes: macro-, micro-, and nano-dimensions. Table 1-1 shows a list of polymeric biomaterials that have been investigated for drug delivery applications. Some of the inherent properties or factors of polymeric biomaterials that can have an effect on their biocompatibility, biodegradability and applicability are: i) physical factors (size and shape), ii) chemical structure and composition of the material, iii) molecular weight distribution, iv) morphology (microstructure, amorphous/crystallinity) of the material, v) physicochemical conditions (ionic strength, $\mathrm{pH}$ ), vi) hydrophilicity/hydrophobicity, vii) water absorption, viii) solubility, ix) lubricity, x) surface energy, xi) degradation and erosion mechanism, and xii) route of administration and site of action [6-10, 23-27, 29].

Although numerous efforts have been made to develop biomaterials for various drug delivery applications, however, still there are unmet medical conditions which require novel materials for delivering drugs to enhance the therapeutic efficacy and patient compliance. The goal of this research was to develop biodegradable polymeric biomaterials in different forms for loading and release of drugs to meet the unmet medical needs.

One of such unmet needs is the efficient and affordable sustained release injectable system for long-acting contraception. Currently, available long-acting reversible contraceptives (LARCs) include intrauterine system (IUS), intrauterine device (IUD), preformed implants, and injectable contraceptives. IUS, IUD, and preformed 
Table 1-1. List of polymeric biomaterials for drug delivery.

\begin{tabular}{lll}
\hline Macro-systems & Micro-systems & Nano-systems \\
\hline Implants & Microspheres & Liposomes \\
Oral delivery systems & Microcapsules & Niosomes \\
(tablets/ capsules/ pumps) & Micelles & Nanospheres \\
In situ depot/implant & Microgels & Solid lipid nanoparticles \\
Hydrogels & Micro-osmotic pump & Nanostructured lipid carriers \\
Transdermal systems & & Polymeric nanoparticles \\
& & Nanogels \\
& & Dendrimers \\
\hline
\end{tabular}


implants require a trained health care provider for implantation and removal, while the injectable LARCs are simple to administer and do not need any surgical procedures, therefore improving patient compliance. Greater than 40 million women worldwide use injectable contraceptives, and their usage is high in developing nations suggesting their need[30]. However, in spite of this popularity, rates of discontinuation are also high for injectable LARCs due to non-adherence. The currently available injectable contraceptives can provide contraception for 1-3 months, thus requiring women to return to their health provider four to 12 times a year, which could be challenging in resource-limited settings of developing nations and may cause discontinuation and prolonged return to fertility due to users' difficulty in complying with the multiple-injection schedule. Despite their effectiveness, presently available LARCs do not address women's needs or specifications which may be due to either poor accessibility or high cost [31-34]. Hence there is a necessity for developing affordable sustained release injectable LARCs that can provide effective contraception for five months or longer after a single shot. This research was focused on developing polyester-based injectable in situ forming depot (ISD) system containing levonorgestrel (LNG) for contraceptive effect for five months or longer after single shot that helps to reduce unintended pregnancies with high patient compliance and low cost and thereby expand contraceptive access and options for women around the globe.

Another unmet drug delivery approach is to enhance the permeability of drugs across the ocular and blood-brain barriers and sustain the release as well. Although there are several potent therapeutics for treating diseases related to eye and brain, their efficacy is still hampered by the lack of an effective method of delivery [35, 36]. Typically, drugs have short half-lives, do not or hardly cross the ocular or blood-brain barriers, and can cause toxicity and side effects at high dose. Nanotechnology shows great promise for transporting therapeutics across biological barriers, reducing drug clearance in the path, and improving the bioavailability of drugs at the target sites [37-43]. Because of these advantages, nanoparticles have become increasingly attractive for delivering drugs to the eye and brain. Most widely investigated nanoparticles for drug delivery across biological barriers (ocular/BBB) were made of polyesters (poly(D,L-glycolide-co-lactide) [44], poly(lactic acid) [45-47], poly( $\varepsilon$-caprolactone)[48, 49]); poly(amino ester)[50], lipid [liposomes [51-54], niosomes [55, 56], solid lipid [57, 58]); polyamines [59-61], polyethyleneimine; polylysine and dendrimers [62-66]; polysaccharide (chitosan [67-70] and hyaluronic acid [70]); polyalkylcyanoacrylate [71-74]; albumin [75-77], and inorganic metals [78]. The polyester nanoparticles have shown to achieve sustained drug release, but not reported to cross the biological barriers themselves without the need to be conjugated or coated with some specific functional moieties [44-49]. Liposomes, solid lipid nanoparticles, dendrimers, surfactant-coated PBCA nanoparticles were reported to be able to cross the barrier; however, they cannot achieve sustained release and also can have toxicity issues $[57,73,79,80]$. The other nanoparticles have not been reported to be able to either sustain drug release or cross the barrier without the need to be conjugated or coated with some specific functional moieties. Therefore, to treat chronic eye and brain diseases such as diabetic retinopathy (DR) and Alzheimer's disease (AD), respectively, there is unmet need of nanoparticles that can carry drugs across the ocular/blood-brain barriers and show sustained release. This work was focused on developing biodegradable 
polysaccharide-based nanoparticles poly(N-isopropyacrylamide-co-dextran-lactate-2hydroxyethyl methacrylate) (Poly(NIPAAm-co-DEXlactateHEMA) nanogels and $\beta$ cyclodextrin-poly ( $\beta$-amino ester) (CD-p-AE) nanoparticles, for sustained release of drugs across ocular and BB barriers, respectively.

\section{Dissertation Hypothesis and Specific Aims}

The central hypothesis of this research was that different biodegradable polymeric biomaterials based on polyesters or polysaccharides can act as effective delivery systems for long-acting contraception for five months or longer, and drug delivery across ocular and blood-brain barriers to treat chronic diseases such as diabetic retinopathy and Alzheimer's disease in the eye and brain, respectively. The central hypothesis of the dissertation has been sub-divided into three sub-hypotheses that drive the formulation of the specific aims of the research:

Sub-hypothesis 1 was that injectable in situ depot system with balanced biodegradable polymers and solvents can be developed as an effective delivery system for sustained release of contraceptives such as levonorgestrel for five months or longer after single SubQ injection through a $23 \mathrm{G}$ needle. This hypothesis was tested in Chapter 2. The specific aims were:

1. Design and conduct in vitro optimization and characterization of LNG-containing ISD formulations.

2. Evaluate in vivo safety, pharmacokinetics and contraceptive effect of LNGcontaining ISD systems in rats.

Sub-hypothesis 2 was that subconjunctivally injectable nanogels composed of DEXlactateHEMA and NIPAAm can cross the ocular barriers for potential delivery of drugs such as insulin to treat diabetic retinopathy. This hypothesis was tested in Chapter 3. The specific aims were:

1. Prepare and characterize poly(NIPAAm-co-DEXlactateHEMA) nanogels.

2. Assess in vitro cytotoxicity, in vitro and ex vivo permeability of the poly(NIPAAm-co-DEXlactateHEMA) nanogels.

Sub-hypothesis 3 was that the nanoparticles composed of $\beta$-cyclodextrin and poly ( $\beta$-amino ester) can enhance the solubility and BBB permeability of Hsp90 inhibitors such as 17-AAG to treat Alzheimer's disease. This hypothesis was tested in Chapter 3. The specific aims were:

1. Prepare and evaluate in vitro drug release of 7-AAG-loaded $\beta$-cyclodextrinpoly ( $\beta$-amino ester) nanoparticles

2 . Investigate in vivo efficacy of $17-\mathrm{AAG}$ loaded $\beta$-cyclodextrin-poly( $\beta$-amino ester) nanoparticles. 


\section{Innovation}

Polyester-based ISD system for long-acting contraception: Although, polyesterbased ISD systems have been one of the successful dosage forms as evidenced by several market products (Eligard $(\mathrm{C}$, Atridox $(\mathrm{C})$ ). However, the major limitations associated with these long-acting ISD systems are injectability and initial burst. Most of these ISD systems are high viscous fluids and use thicker-gauge needle (such as 18G/19G) for injection and this limits the patient compliance. Attempts made to lower the viscosity of the fluids would usually result in an increase in the initial burst and may also lower the ability for long term release. In this research, unique strategies were employed to improve the injectability and initial burst profile, which include the use PLGA, PLA with different biodegradable properties, and blends of these polyesters; use co-solvents of NMP, TEC, benzyl benzoate (BB), and vary the polymer/solvent ratios. These strategies helped to improve the injectability while lowering the initial burst. This resulted in a unique injectable ISD system containing LNG for long-acting contraception, which can be injected through a thinner-gauge needle $(21-23 \mathrm{G})$ and has an advantage of low cost, the simplicity of manufacture, ease of application to the body, easy to hide, versatility and complete biodegradability. The use of approved materials, such as PLA, PLGA, and solvents used in this study can lessen the product approval time for future clinical trials. This system stands as an attractive substitute to conventional preformed implants and microparticles for parenteral sustained release applications.

Polysaccharide-based nanoparticles for drug delivery to the eye and brain i) Poly(NIPAAm-co-DEXlactateHEMA) nanogels for ocular drug delivery: Biodegradable and hydrophilic nature of the dextran polysaccharide were slightly modified by grafting with hydrophobic hydrolytic degradable PLA and then copolymerized with thermoresponsive poly-NIPAAm and acrylic acid using emulsion photopolymerization to make nanogels that possess both thermoresponsive, biodegradable and charge properties. The strategy behind designing poly(NIPAAm-coDEXlactateHEMA) based nanogel delivery system is to combine the merits of biodegradable nature and nano-sized particulate properties. The degradation property of the nanogels matrix can be used to modulate the release of encapsulated drugs for an extended period of time and helps to avoid the removal of the empty device after treatment. The nanogels will be thermoresponsive and exhibit a phase transition above lower critical solution temperature $\left(\mathrm{LCST} \sim 32^{\circ} \mathrm{C}\right)$ and becomes more hydrophobic because of the strong hydrophobic interactions between the Poly-NIPAAm chains. The thermoresponsive property of the nanogels makes them more hydrophobic at a physiological temperature and this slows down the water penetration into the system and thereby lowers the degradation rate and controls the drug release. Meanwhile, the nanosized dimensions impart the advantages of nanoparticulate systems such as transporting drugs across biological barriers, improving drug stability and bioavailability at the targets $[18,37,81-86]$. Additionally, incorporation of carboxylic functional groups through the addition of acrylic acid imparts charge onto the nanoparticles which can assist in the internalization of the nanoparticles and can be used for further conjugation with a ligand. Hence, in an attempt to minimize the constraints and disadvantages of conventional 
ocular drug delivery systems, a contemporary poly(NIPAAm-co-DEXlactateHEMA) nanogels based sustained drug delivery systems could be very promising.

ii) $\beta$-cyclodextrin-poly( $\beta$-amino ester) (CD-p-AE) nanoparticles for brain delivery: This polysaccharide-based biomaterial ( $\beta$-cyclodextrin $(\mathrm{CD})$-based nanoparticles) was formed by combining poly( $\beta$-amino ester), which contains ester bond and amine units with acrylated $\beta$-CD. CD-p-AE nanoparticles are cross-linked, hydrolytically degradable, and catatonically charged, and contains $\beta$-CD units. Incorporation of $\beta$-cyclodextrin $(\beta-C D)$ into the nanoparticle structure facilitates the loading of hydrophobic drugs and thereby expected to improve the solubility of the hydrophobic drugs. The biodegradable property of poly( $\beta$-amino ester) can be tuned to control the release rate. Introducing cationic charge onto the nanoparticles was considered as an effective approach to enhance the permeability across BBB $[87,88]$.

\section{Dissertation Outline}

Different types of biodegradable polymeric biomaterials based on polyesters or polysaccharides were developed for loading and release of drugs to meet the unmet medical needs. Basic preliminary preclinical proof of concept studies were also conducted to investigate the potential applicability of the developed biomaterials in their designed applications.

Chapter 2 tested the sub-hypothesis 1 that injectable in situ depot system with balanced biodegradable polymers and solvents can be developed as an effective delivery system for sustained release of contraceptives such as levonorgestrel for five months or longer after single SubQ injection through a $23 \mathrm{G}$ needle. The background section of this chapters covers a comprehensive literature review on injectable long-acting contraceptives. A series of ISD formulations were designed by employing unique strategies which include the use PLGA, PLA with different biodegradable properties, and blends of these polyesters, use solvent mixtures of NMP, TEC, BB, and vary the polymer/solvent ratios, and various drug loadings. The formulations are evaluated for viscosity, initial burst, in vitro and in vivo long-term release. The effect of different formulation parameters such as polymer concentration, polymer inherent viscosity, drug loading and solvent combination on the viscosity, injectability, in vitro release, in vivo initial burst of drug from ISD systems was evaluated. By tailoring the formulations parameters, ISD system with suitable injectability and initial burst was obtained. Future directions evolving from this work were suggested at the end of the section.

Chapter 3 tested the objective that the polysaccharide-based nanoparticles with the tailored balance of hydrophilicity/hydrophobicity, and charge content can be used to assist the drugs to cross biological barriers such as ocular and blood-brain barriers and sustain the release as well. Depending on the need of the therapeutic application, two types of polysaccharide-based nanoparticles were investigated for their drug delivery feasibility which includes: (a) poly(NIPAAm-co-DEXlactateHEMA) nanogels for the potential delivery of hydrophilic peptide (insulin) across ocular barriers for the treatment 
of Diabetic Retinopathy. (b) $\beta$-cyclodextrin-poly( $\beta$-amino ester) nanoparticles as potential drug carriers to enhance the solubility and BBB permeability of 17-AAG to treat Alzheimer's disease. Future directions pertaining to this work were recommended at the end of the chapter.

Chapter 4 describes the conclusions of the thesis, identifying the end results of this work in relation to the overall goal of the thesis.

In the Appendix (Tables A-1 through A-5, and Figures A-1 through A-5), complete details of the study testing the impact of ionizable modifiers 2(Dimethylamino)ethyl methacrylate (DMAEMA) on mechanical, thermal and hydration properties of the Poly(HEMA)-based hydrogels was given. The addition of ionic species such as DMAEMA at low mol\% (5 mol\%) into poly(HEMA) hydrogels has significantly altered the mechanical, thermal and hydration properties of the hydrogels. Hydration properties can be altered by the hydrophobicity/ hydrophilicity \& hydrogen bond forming ability of the added cationic modifiers. 


\section{CHAPTER 2. INJECTABLE IN SITU FORMING DEPOT SYSTEMS FOR LONG-ACTING CONTRACEPTION*}

\section{Background}

\section{Long-acting Reversible Contraceptives}

Birth control is still a major concern worldwide. The most common approach to controlling fertility to prevent unintended pregnancy is oral administration of birth control pills. However, this method suffers from limitations such as daily ingestion of pills, needs continuous user attention, and daily variations in blood concentrations of the drug leading to unwanted side effects [89]. Alternatively, a long-term, systemic, controlled delivery of contraceptive drugs is beneficial, and is gaining attention in preventing pregnancy, as well as in postmenopausal therapy because of the advantages such as: convenient and don't require daily action, provides continuity of drug supply, avoids the variability in absorption and metabolism associated with oral therapy, minimize the fluctuations in blood concentration thereby lowering the side effects, and cost effective on overall usage [90,91]. Further, there is less chance of over/under-dosing with the sustained release dosage form regimen, and patient compliance with a multi-day, -week, or - month sustained release regimen is superior to frequent oral dosing.

Long-acting reversible contraceptives (LARCs) are birth control methods that provide contraception over a prolonged period (months to years) without the need of user activity and are proven to be most effective in lowering the rates of unintended pregnancies [92]. There is a wide gap in terms of therapeutic duration among the currently available LARCs on the market. LARCs based on preformed implants that are available on the market such as Implanon ${ }^{\circledR}$, Nexplanaon ${ }^{\circledR}$, Norplant ${ }^{\circledR}$, Implanon ${ }^{\circledR}$ and Sino-Implant ${ }^{\circledR}$ offer extended and effective contraception for up to 3-7 years. However, such long duration is undesirable for some women, and these preformed implants are not

* Parts of this chapter were adapted or reprinted with permission from (i) "Pharmaceutical research. Long-acting injectable hormonal dosage forms for contraception. 32(7). 2015. p. 2180-2191. Wu, L., Janagam, D. R., Mandrell, T.D., Johnson, J.R., Lowe, T.L. (C) Springer Science + Business Media New York 2015. With permission of Springer." (ii) "Development and validation of sensitive LC/MS/MS method for quantitative bioanalysis of levonorgestrel in rat plasma and application to pharmacokinetics study. 1003. Ananthula, S., Janagam, D. R., Jamalapuram, S., Johnson, J. R., Mandrell, T. D., Lowe, T. L. Journal of Chromatography B. p. 47-53. (C) 2015 Elsevier B.V., with permission from Elsevier." (iii) "Janagam, D. R., Wang, L., Ananthula, S., Johnson, J. R., Lowe, T. L. (2016). An accelerated release study to evaluate long-acting contraceptive levonorgestrel-containing in situ forming depot systems. Pharmaceutics, 8(3), 28. C 2016 by the authors; licensee MDPI.” 
biodegradable, so they need to be removed by an experienced clinician at the end of the treatment [93]. On the other hand, the injectable LARCs $\left(\right.$ Cyclofem $^{\circledR}$, Lunelle $^{\circledR}$ and Depo-Provera ${ }^{\circledR}$ ), which are aqueous suspensions of drug microcrystals, can provide contraception for 1-3 months and this short term contraception can increase flexibility to women [94]. Injectable LARCs are increasingly used for preventing unintended pregnancies as they are reversible, can be used on-demand, easy to use and do not require surgical implantation and removal. They have shown 96\% effectiveness with a typical use [94]. More than 40 million women worldwide use injectable contraceptives [30]. However, because of the short reinjection intervals, the current choice of injectable contraceptives require women to return to their health-care provider for at least four to 12 times a year [31]. This frequent clinic visits could result in discontinuation due to users' difficulty in complying with the multiple injection schedules, especially in resourcelimited areas in developing nations. In spite of their high popularity, typical use brings about much lower adequacy. In substantial part, this difference reflects challenges in adherence to the preventive regimen and low rates for long-term continuation. Additionally, presently available long-acting contraceptives do not address women's needs or specifications which may be due to either low compliance, poor accessibility or high cost [31-34]. Therefore, there is still an unmet need for injectable contraceptives which can provide contraception for five months and up to one year after a single injection so that better adherence and continuation rates can be achieved $[31,34]$. Including this new alternative into the current contraceptive choices will offer great flexibility, expand contraceptive prevalence, and decrease the burden of patient load on small clinical facilities and community-based programs [31].

\section{Injectable Long-acting Reversible Contraceptives}

In recognition of the potential advantages, significant efforts have been made in the past decades to develop injectable LARC dosage forms. Among those, the most common injectable dosage forms that were explored to achieve control release of contraceptive steroids are drug microcrystal suspensions, drug-loaded microsphere suspensions, and in situ forming depot (ISD) systems [95-98]. Each dosage form has its advantages and limitations which are summarized in Figure 2-1. However, only drug microcrystal suspension based dosage forms (Depo-Provera ${ }^{\circledR}$ and Depo-subQ Provera $104^{\circledR}$ ) are commercially available on the market for 3-month contraceptive protection. So far no microsphere or ISD based product has been approved for contraception.

Drug microcrystals have advantages of low cost due to fewer process steps and materials involved, and using water as an administration vehicle, but needs reconstitution and are difficult to achieve sustained release of APIs for more than three months. Microspheres have the advantages of using water as a vehicle, and capability of achieving more than the three-month release of APIs due to the retarding effect of the polymeric matrix [99-109]. However, more often the microencapsulation processes involve the use of high temperature and organic solvents which may affect the stability of the therapeutics, and some toxic organic solvents might be trapped inside. Manufacturing can be difficult to produce on a large scale and is generally costly for well-defined 


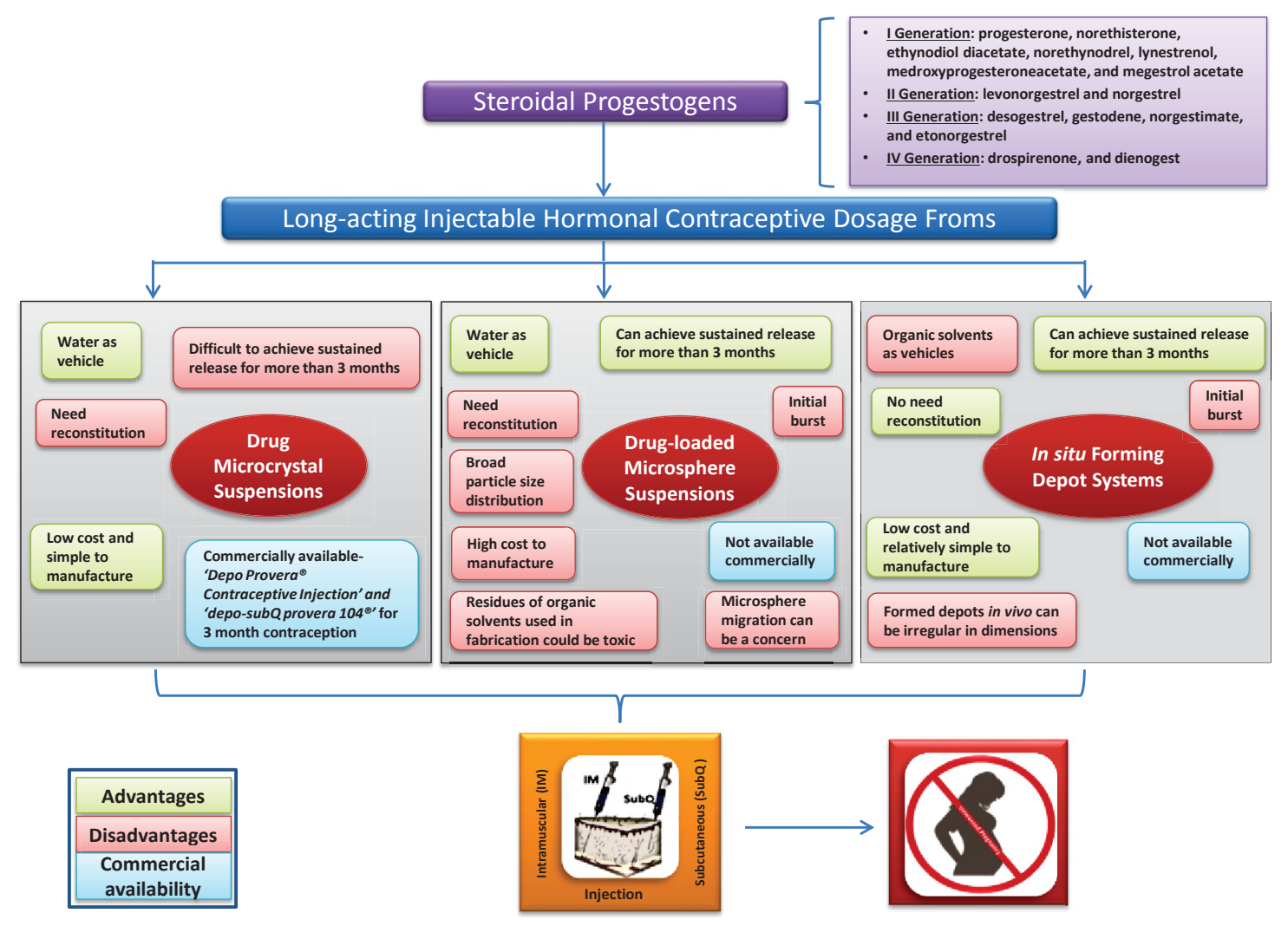

Figure 2-1. Flow chart of the advantages, disadvantages, and commercial availability of injectable LARCs.

Notes: Drug microcrystal suspensions, drug-loaded microsphere suspensions and in situ forming depot systems (ISD) are the widely studied injectable hormonal long-acting reversible contraceptive (LARC) dosage forms. 
microspheres. Their storage often presents problems, and also need to be reconstituted at the time of injection. Because of the particle nature, microspheres fail to form a preferred continuous and nearly homogeneous, monolithic drug depot matrix. The possibility of microsphere migration from the site of injection might also be a concern [96]. In situ forming depots have advantages of simplicity of manufacture, no need for reconstitution, and versatility. However, they have disadvantages of using organic solvents as vehicles, forming depots in vivo with non-precise shapes and sizes, and requiring drugs with broad therapeutic windows [97, 110].

\section{Drug Microcrystal Suspension}

The components in a drug microcrystal suspension dosage form are only API microcrystals and vehicle for injection (water). Thus, this dosage form is simple, easy and low cost to be manufactured. The microcrystal suspension dosage form can achieve longer therapeutic effects than the API alone (non-crystal form) due to the slow dissolution of the API from the drug crystals into the body fluid. When prodrugs are used to form the drug microcrystals, the combination of dissolution of the prodrugs from the crystals and subsequent hydrolysis/cleavage of the prodrugs into active entities controls the release of the API into the body fluid. Considerable efforts have been made to develop injectable suspensions of contraceptive steroidal progestogen microcrystals containing either the active drug or prodrug obtained via esterification of the hydroxyl groups of the active drug. Depending on the contraceptive API types and microcrystal sizes, up to three-month contraception can be achieved [111, 112]. For examples, medroxyprogesterone acetate, the most well-known steroid drug, has been formulated into injectable microcrystals for three-month contraceptive protection. It has been marketed as Depo-Provera ${ }^{\circledR}$ and Depo-subQ Provera $104^{\circledR}$ administrated by intramuscular and subcutaneous injections, respectively. Norethisterone enanthate, an ester of norethisterone (NET), has been developed as injectable microcrystals for twomonth contraception $[112,113]$. Levonorgestrel has been formulated into prodrugs levonorgestrel cyclobutylcarboxylate and levonorgestrel butanoate (LNG-B) which have lower water solubility than LNG and have been studied in rats, rhesus and cynomolgus monkeys, and women [98]. Both the prodrugs could suppress ovulation for 5-6 months in women at a single dose of $50 \mathrm{mg}$ in the form of microcrystal suspensions. LNG-B demonstrated better performance in terms of overall toxicity, pharmacokinetic profiles and pharmacodynamics effects in phase I clinical trials [98, 114]. Currently, several companies are developing LNG-B crystal suspensions for long-acting contraceptives $[115,116]$. For example, CONRAD is trying to formulate LNG-B for preclinical and clinical studies aiming at contraception for 4 months after a single injection [116].

\section{Polymer-based Drug Delivery Systems}

As discussed in the introduction (chapter 1), a significant emphasis was made in the recent years on innovation for improving the delivery of a drug by designing various polymer-based drug delivery systems. Polyester-based drug delivery systems have been extensively investigated for the long-term controlled delivery of contraceptive steroids [31]. Polyesters are the earliest and most widely investigated class of synthetic 
biodegradable polymers for drug delivery applications and comprehensively reviewed [12-17, 117]. These are thermoplastic polymers with hydrolytically sensitive aliphatic ester linkages in the backbone. Poly( $\varepsilon$-caprolactone)(PCL), poly(lactic acid)(PLA), poly(glycolic acid)(PGA) and their copolymers poly(lactic acid-co-glycolic acid)(PLGA) are some of the well-defined biomaterials with regards to design and performance [7]. Because of their adjustable physicochemical properties such as swelling and biodegradation kinetics, molecular interaction potential with embedded therapeutics, they offered several possibilities in the design of new drug delivery systems [118-121]. The common synthetic scheme to make poly(esters) is by ring-opening polymerization from the corresponding cyclic lactone monomers (shown in Figure 2-2). Polyesters predominantly undergo bulk erosion where the polymeric matrices degrade over the entire cross-section and show non-linear erosion kinetics [122]. Several reviews have been published on their degradation mechanisms [10, 122-127]. The major advantage of biodegradable polyester based drug delivery systems is that they provide a continuous delivery of the drug via diffusion or by polymer erosion to surrounding tissues, minimizing the variations in blood concentrations of the drug and offers maximum therapeutic efficiency at a minimum drug dose [128]. Most extensively studied injectable polymeric systems for long-term release of contraceptive include i) Polymer-based drug microspheres and ii) Injectable in situ forming depot (ISD) systems.

\section{Drug microsphere suspensions}

Microspheres made of polymers have the advantage of using water as a vehicle to form suspensions for long term (over months) sustained release of different drugs [129134]. Although no microsphere-based product was approved for contraception, longacting leuprolide injections are currently sold in the forms of both microspheres (LUPRON DEPOT ${ }^{\circledR}$ ). Starting in the late $70 \mathrm{~s}$, injectable microsphere contraceptive systems have been developed for long-term contraception, pioneered by Beck and coworkers using PLA (MW 90,000 Da), and/or PLGA (96:4, 92:8, 87:13 and 74:26; MW about $40,000 \mathrm{Da})$ to encapsulate NET via oil-in-water $(\mathrm{o} / \mathrm{w})$ emulsion/solvent evaporation process [135]. NET microcrystals were successfully encapsulated into PLA and PLGA microspheres by o/w emulsion and solvent evaporation technique. Afterwards, PLGA became the most popular polymer for fabricating microspheres to encapsulate contraceptive APIs including LNG, gestodene and ethinyl estradiol using o/w or w/o/w emulsion/solvent evaporation technique [99-102, 104, 106, 136, 137]. PCL is a second popular polymer for fabricating microspheres to encapsulate contraceptive APIs including LNG, ethinylestradiol, norgestrel, progesterone and $\beta$-estradiol using $\mathrm{o} / \mathrm{W}$ or $\mathrm{w} / \mathrm{o} / \mathrm{w}$ emulsion/solvent evaporation technique [138-143]. The quality of microspheres depended on emulsion types, solvents, temperature, and surfactants/stabilizers. Through extensive investigation over past several decades, it has been acknowledged that many factors of microspheres such as their chemical and physical structure (composition, chirality and crosslinking density), drug loading and distribution, physical property (size, shape, size distribution, and molecular weight), and fabrication and sterilization processes significantly affects drug release profiles including release rate, duration of action and initial burst, and microsphere overall performance in vitro, in animals, and in humans $[99,102,106,135,136,141,144-146]$. 


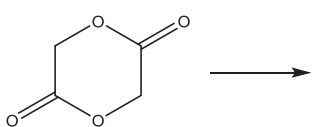

Glycolide



Lactide

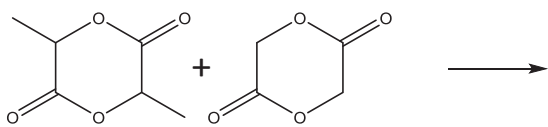

Glycolide
Lactide

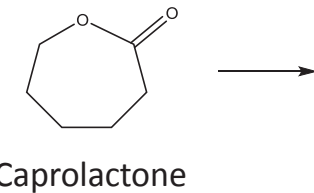

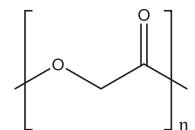

Poly(glycolic acid)

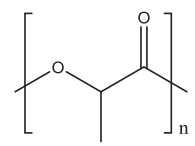

Poly(lactic acid)

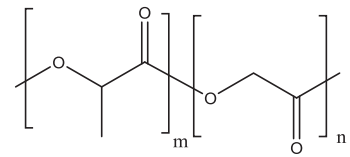

Poly(lactic-co-glycolic acid)

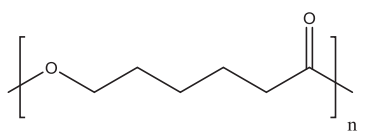

Poly(caprolactone)

Figure 2-2. Ring opening polymerization of selected cyclic lactones to give the corresponding polyester polymers. 
The composition of the microspheres controls the biodegradation rate of the microspheres, and in turn, affects the contraceptive API release profiles from the microspheres. For example, as the hydrolytic degradation rate of the polymers PGA, PLA and PCL increased in the order of PGA $>$ PLA $>>$ PCL, the microspheres made of the triblock polymers consisting these three polymer blocks released LNG at a rate increasing with increasing the amount of the three polymer blocks in the order of PGA $>$ PLA $>>$ PCL [147]. PCL possessed a good permeability for steroidal drugs but undergoes slow biodegradation, resulting in the release of LNG from the PCL matrix to be controlled by diffusion [147-149]. On the other hand, PLA and PGA had a lower permeability to steroidal drugs but undergo homogeneous erosion, resulting in the release of LNG from the PCL matrix to be controlled mainly by erosion $[147,150]$. The chirality of polymers, crosslinking density and drug loading of microspheres also play important roles in controlling contraceptive API release profiles. It was reported that microspheres composed of D, L-lactide caused more uniform release rate and less initial burst for progesterone and $\beta$-estradiol than those composed of L-lactide [141]. Microspheres with a higher amount of unencapsulated drug present on the surface would show higher initial burst $[99,136,138]$. When fabricated microspheres were not uniform, initial burst release was observed in the in vitro, in vivo and clinical evaluations of the microspheres for contraception $[100,101,106,140,143,151,152]$. Even though this initial burst release might be not very harmful to the users, it might cause API concentration beyond the therapeutic window and reduce the amount of API available for contraception and the duration of release [153-155]. Among the methods studied for minimizing the initial burst release, coating or core/shell structure is one of the most straightforward strategies $[156,157]$. This additional layer of the shell could be created by a dipping, mixing or emulsion process [132]. It was reported that simply modifying the prototype NET-loaded PLGA microspheres by adding a polymer/chloroform solution into the suspension of the prototype microspheres in alcohol aqueous solution significantly reduced the initial burst and extended the release duration of NET for 40-50 days longer than the prototype microspheres with a similar size range and NET loading [158]. The reduced initial burst and extended release duration of NET from the modified microspheres was because the polymer coating layer added an additional barrier for the drug to diffuse/penetrate through.

Microsphere size is effective means for modulating contraceptive API release kinetics $[99,135,146]$ and as well as microsphere injectability [99]. Primarily, the size of microspheres determines the surface area to volume ratio, and thus directs the extent of surface available for releasing the contraceptive APIs through diffusion. Smaller microspheres have higher surface area to volume ratio and less diffusion path length for the APIs, and subsequently, result in quicker API release rate and shorter release duration. This effect was demonstrated in a study where the release rate of NET and progesterone increased with decreased size of PLGA [106] and chitosan [159] microspheres, respectively. The size of microspheres inversely affects the efficiency of the injectability: the bigger the size is, the more difficult the microspheres can be injected through clinically used needles. The optimal size range for yield and efficiency of injection is between 20 and $90 \mu \mathrm{m}$ [99]. The mean size of microspheres can be controlled by the concentration of polymers used for fabricating the microspheres. The higher the 
polymer concentration is, the higher the viscosity of the organic phase in emulsion droplets is, and thus the bigger the mean size is and the more contraceptive APIs are encapsulated into the microspheres $[138,142]$. The molecular weight (MW) of the polymers used for fabricating microspheres is another important factor affecting contraceptive API release. The higher is the MW, the slower the polymers degrade and the longer the APIs are released [147]. For example, microspheres composed of PLGA with lower MW (12,000 Da) led to 35 day release of ethinyl estradiol, while PLGA with higher MW (70,000 Da) resulted in 105 day release of ethinyl estradiol [101, 102].

Female baboons, rats, rabbits and mice have been used as animal models to evaluate serum API levels and contraceptive effect of microspheres after single intramuscular injection. Beck and co-workers evaluated their NET-loaded microspheres on female baboons. Their PLA microspheres $(10-240 \mu \mathrm{m})$ could sustain the release of NET in female baboons for about 180 days, and the ovulation was inhibited through the 6-month treatment period for all three dose levels tested: 75, 50, $25 \mathrm{mg}$ of NET equivalent [135]. In vivo evaluation of their NET-loaded PLGA microspheres on baboons showed that the NET release duration was dependent on the particle size and the LA to GA ratios of PLGA polymers, and the release of NET presented a two-phase pattern [106]. Beck and co-workers also designed LNG-loaded PLGA(87:13) microspheres and found that the microspheres released LNG in baboons for up to 6 months with 3-6 month ovarian function suppression, depending on the dose level [99]. A biphasic release pattern was seen in LNG release from the microspheres in a similar way as the NET-loaded microsphere systems. In rat studies, LNG- or LNG and ethinylestradiol-loaded PLGA and PCL microspheres were found to maintain constant LNG blood level at $0.2-2 \mathrm{ng} / \mathrm{mL}$ for 15 weeks to 5 months $[100,101,143]$. In rabbit studies, it was found that progesterone was maintained a blood level of $1-2 \mathrm{ng} / \mathrm{mL}$ for about 5 months [159], 5 months [160] and 20 days [161] by microspheres made of chitosan, casein and serum albumin, respectively [159, 160]; and LNG was maintained a blood level of 0.1-0.5 $\mathrm{ng} / \mathrm{mL}$ for 28 days by microspheres made of casein [104]. In mouse study, LNG- or LNG and estradiol-loaded poly(ethylene glycol-b-poly(D,L-lactide)) [162], gelatin [163] or poly(3-hydroxy butyrate) [164] microspheres showed 6 month, 50 day, and 1-3 month contraceptive effect, respectively. Phases I, II and II clinical trials have been conducted for the NET-loaded PLA and PLGA microspheres [107-109, 152, 165]. The phase I clinical trials [165] of these NET-loaded PLA microspheres (size range: $60-240 \mu \mathrm{m}$, or 90-212 $\mu \mathrm{m}$ ) in 63 women at three centers showed that the release of NET lasted for 6 months after one intramuscular injection, while the serum levels of NET varied in proportion to the dose injected and the suppression of ovarian function was also highly dose dependent. Suppression of ovulation for 6 months could be achieved for doses ranging from 1.33 to $3.45 \mathrm{mg}$ of NET/ $\mathrm{kg}$ for NET-loaded PLA microspheres with size 60-240 $\mu \mathrm{m}$ [106]. In order to avoid the build-up of PLA at the injection sites due to its slow degradation, Beck and coworkers designed the second generation NET microspheres using PLGA [152]. NET-loaded PLGA microspheres (63-90 $\mu \mathrm{m}$ or 90-106 $\mu \mathrm{m}$ ) fabricated from PLGA with $86 \%-88 \%$ LA and 14\%-12\% GA were clinically tested in women. Microspheres with sizes of 63-90 $\mu \mathrm{m}$ could suppress ovarian function for 3-4 months at dose of $75 \mathrm{mg}$ or $100 \mathrm{mg}$ of NET, while the $90-106 \mu \mathrm{m}$ microspheres 
suppressed ovarian cyclicity for an average of 22 weeks with the shortest one of 14 weeks and longest one of 26 weeks at similar dose levels [166, 167].

It would be reasonable to assume that well-defined polyester microspheres with core/shell structures and narrow particle size distribution could be fabricated to encapsulate the contraceptive APIs for reduced initial burst release and extended contraception period, although no attempts have been made in this direction.

\section{Injectable in situ forming depot systems}

The ability to inject a combination of drug and a biodegradable polymer in liquid form to a localized site and have the biodegradable polymer form a depot matrix entrapping the drug has a number of advantages. Some of these advantages include $100 \%$ loading efficiency, easy administration, few processing steps and relatively simple manufacturing process, potential for low cost, no need for reconstitution, prolonged delivery, completely biodegradable, and no need for surgical removal at the end of the treatment [168-172]. Because of these advantages, ISD systems have emerged as an attractive alternative to the preformed implants and microparticles in the field of parenteral sustained release formulations [170]. Other common terms that are used in literature for defining these systems include in situ forming implants or in situ gelling systems.

ISD formulations are liquids before injection and transform in the body to a gel or solid depot at the site of injection. Various mechanisms can be used to trigger this solidification process which includes phase separation induced by $\mathrm{pH}$ [173-175], solvent exchange [176, 177] or temperature [178], and physical or chemical crosslinking [179], in situ solidifying organogels [180]. However, the phase separation based ISD systems involving solvent exchange have attracted considerable attention from the pharmaceutical industry because of its advantages compared to its counterparts, as they do not depend on critical temperature (for temperature-sensitive ISD systems), change in $\mathrm{pH}$ (for $\mathrm{pH}-$ sensitive ISD systems), presence of ions (for charge-sensitive ISD systems) for the phase separation to form a depot [181]. Moreover, commercially available raw materials can be used for the development of these formulations $(30,99,100)$.

Solvent exchange based ISD formulation is a solution or suspension containing drug and biodegradable polymers dissolved or dispersed in pharmaceutically acceptable water-miscible organic solvents, respectively [31, 170, 171, 182-184]. Upon injection into the tissue, this ISD system forms a solid depot of polymeric matrix at the injection site because of the phase separation (in situ polymer precipitation) by solvent exchange, as the water-miscible organic solvent diffuses into the surrounding aqueous medium while the aqueous body fluid penetrates into the organic phase $[170,181,184,185]$. The drug entrapped in the depot is then slowly released out into the surrounding body fluid due to the degradation of the polymers and diffusion of the drug, goes into the systemic circulation and then reaches target sites. The biodegradable polymers will eventually completely degrade at the injection site over a period, and be cleared out from the body. Because of the complete biodegradation, the ISD system does not need any surgical 
removal at the end of the treatment. This phenomenon of phase transition is explained by several other terms in the literature which include: "solvent induced phase inversion [181], in situ polymer precipitation [186, 187], solvent exchange [188], solvent/nonsolvent exchange [189], solvent removal [190], solvent-removal precipitation [186], nonsolvent induced phase separation [191], and liquid-liquid phase separation [192]”.

Table 2-1 lists out the common solvents/plasticizers used in ISD formulations. The ISD technology has been developed for safe and efficient delivery of drugs in vivo for a various period of weeks to months $[31,170,183,193]$, to treat cancer, infections, hormonal disorders, pain, immunomodulation, neurological disorders and metabolic disorders $[170,194]$. Several ISD systems are currently available as marketed products such as Eligard ${ }^{\circledR}$ and Atridox ${ }^{\circledR}[170]$. However, as mentioned earlier no ISD product is available for contraception.

Dunn et al. pioneered this concept in 1990 by employing biodegradable polyesters such as PLGA and PLA in water-miscible organic solvents such as NMP and DMSO $(95,99,101)$. The concept of in situ depot formation was soon adopted to develop longacting injectable contraceptives $(102,103)$. Dr. Shukla's and Dr. Johnson's group from the University of Tennessee Health Science Center (UTHSC) has been actively involved in the development of ISD formulations for sustained release of contraceptive APIs such as LNG and ethinylestradiol for long-acting contraception [195-197].

The studies were started with Precirol ATO 5 as a depot matrix, Labrafil 1944 CS as a solvent/plasticizer and LNG as an API for contraceptive [198]. The injectability of such systems was strongly dependent on the Precirol concentration and the size of the needle used. Lower maximum force for injection was observed for lower Precirol concentrations and larger gauge needles. The same trend was observed for the ISD systems developed from PLGA/PLA [199]. In vitro release kinetics demonstrated that the release rate of LNG from the resulting depots was affected by the Precirol concentrations used in the formulation and the LNG crystal sizes [198]. Moreover, zero order release of LNG was achieved up to 30 days in vitro for formulations containing $10 \mathrm{wt} \%$ Precirol, and $2 \mathrm{wt} \% \mathrm{LNG}$ with drug crystal size of $6 \mu \mathrm{m}$ [198]. In vivo investigation demonstrated that the durations of suppression on rat estrous cycles after single subcutaneous (SubQ) injection were significantly influenced by the LNG loading in the $10 \mathrm{wt} \%$ Precirol formulation with 20, 27 and 41 consecutive days for rats receiving $1 \mathrm{~mL}$ of the formulations containing $0.25,0.50$ and $2.00 \mathrm{wt} \%$ LNG loading, respectively [198]. These short contraception durations might be attributed to the fast degradation of the formed depots which disappeared in the injection sites around 35 to 45 days post-injection [198].

In order to achieve longer contraception protection after a single injection, polyesters such as PLGA (various ratios of LA to GA) and PLA with a different intrinsic viscosity (molecular weight) were evaluated as the depot matrix [199, 200]. The effects of varying drug loading, polymer concentration, polymer inherent viscosity and copolymer composition on in vitro release of LNG were studied. Increasing the LNG loading from 1 to $8 \% \mathrm{w} / \mathrm{w}$ increased the cumulative amount of LNG released in vitro on day 30 from 1.195 to $3.4045 \mathrm{mg}$ per gram of formulation made from PLGA50:50. The cumulative amount of LNG released on day 30 decreased from 2.06 to $0.628 \mathrm{mg}$ per 
Table 2-1. Common solvents (plasticizers) and their key features.

\begin{tabular}{|c|c|c|c|}
\hline Solvent & Structure & $\begin{array}{c}\text { Viscosity (cP @ } \\
\left.25^{\circ} \mathrm{C}\right)\end{array}$ & $\begin{array}{c}\text { Water miscibility } \\
(\mathrm{mg} / \mathrm{mL})\end{array}$ \\
\hline $\begin{array}{l}\text { N- methyl-2- } \\
\text { pyrrolidone (NMP) }\end{array}$ & & 1.65 [157] & 1000 [201] \\
\hline $\begin{array}{l}\text { Demthyl sulfoxide } \\
\text { (DMSO) }\end{array}$ & & $1.99[155]$ & miscible [202] \\
\hline $\begin{array}{l}\text { Benzyl benzoate } \\
\text { (BB) }\end{array}$ & & $8.292[203]$ & $\begin{array}{c}\text { practically } \\
\text { insoluble [201] }\end{array}$ \\
\hline $\begin{array}{l}\text { Triethylcitrate } \\
\text { (TEC) }\end{array}$ & & $35.2[156]$ & 57 [175] \\
\hline $\begin{array}{l}\text { Benzyl alcohol } \\
\text { (BA) }\end{array}$ & & $5.05[204]$ & $35[163]$ \\
\hline Glycofurol & & $8-18$ [160] & miscible [202] \\
\hline Triacetin & & $17[164]$ & 64 [201] \\
\hline Ethyl Acetate (EA) & & 0.45 [169] & 82.3 [205] \\
\hline
\end{tabular}


gram of formulation when PLGA50:50 concentration increased from 20 to $80 \% \mathrm{w} / \mathrm{w}$. Increasing the PLGA50:50 inherent viscosity from 0.15 to $1.07 \mathrm{dL} \cdot \mathrm{g}-1$ decreased the cumulative amount of drug released on day 30 from 14.17 to $0.935 \mathrm{mg}$ per gram of formulation. In vitro drug release rate was found to decrease with increasing proportion of lactide in lactide-glycolide copolymers. Drug release from the system was due to a combination of diffusion and polymer degradation. ISD systems prepared from PLGA50:50/NMP (33:67 w/w) with different LNG contents were further investigated in vivo on Sprague-Dawley albino female rats for contraceptive effect after SubQ injection. The duration of in vivo activity of the LNG released from the in situ formed depots was evaluated by monitoring the estrous cycles of the female rats. The sizes of the in situ formed depots decreased with time, and the depots disappeared completely from the injection site between day 45 and 70 after injection. Formulations containing 2 and $4 \%$ $\mathrm{w} / \mathrm{w}$ LNG suppressed the rat normal estrous cycles for 65.5 and $103.5 \pm 8.5$ days at a dose level of $1 \mathrm{ml} \cdot \mathrm{kg}^{-1}$, respectively $[199,200]$. The formulation containing $2 \mathrm{wt} \%$ of LNG in $32.67 \mathrm{wt} \%$ PLGA (inherent viscosity of 0.59 ) and $65.34 \mathrm{w} \% \mathrm{NMP}$ was further evaluated on Japanese quails with Depo-Provera ${ }^{\circledR}$ as a control [206]. The dose level was $40 \mathrm{mg}$ API equivalent per kg and the injection site was the left pectoral musculature. Quails treated with the LNG-containing ISD system stopped laying eggs for $67 \pm 4.1$ days, while resume ovarian recrudescence (evidenced by egg laying) on day 59 after treatment (one bird; range, 59-70 days). For the birds treated with Depo-Provera ${ }^{\circledR}$, two of seven birds stopped egg-laying for 19 and 49 days, respectively, while for the other five birds, the average duration of not egg-laying was $5.8 \pm 2.3$ days (range, $2-8$ days). The contraceptive duration observed on these quail was consistent with that observed in rats for the same formulation $[199,206]$. The formulation containing $4 \mathrm{wt} \%$ LNG was further evaluated at the dose level of $40 \mathrm{mg} / \mathrm{kg}$ body weight (equivalent to $1 \mathrm{~mL} \cdot \mathrm{kg}^{-1}$ body weight) on Feline Queens via subcutaneous injection [207]. Blood and fecal samples were collected weekly for the first 5 weeks to build the baselines. Initial treatment injections were given at the beginning of week 6 , and all queens received a second injection with the same dosage at the beginning of week 16 (68 days after initial treatment). The results demonstrated that the follicular activity and estrogen secretion in the domestic cats could be suppressed for at least 36 weeks after the two injections of the formulation [207].

Based on the above in vivo evaluations, cottontop tamarins, a small new world nonhuman primate, were used to further evaluate the contraceptive effect of the PLGAbased formulations [208]. The formulation was slightly modified to contain $24 \mathrm{wt} \%$ PLGA50:50 as the depot matrix and $72 \mathrm{wt} \% \mathrm{NMP} /$ triethyl citrate (TEC) (90:10, v:v) as the solvent, and denoted as F1. The F1 formulation was subcutaneously injected into cottontop tamarins at an LNG dose level of $45.53 \pm 2.52 \mathrm{mg} / \mathrm{kg}$. The normal ovarian cycle of the cottontop tamarin was suppressed for $102.0 \pm 20.9$ days indicated by the fecal $\mathrm{PdG}$ and E1C concentrations. In order to extend the suppression duration, the type and intrinsic viscosity of the polyester used in the formulation were adjusted to increase the viscosity of the formulation and slow the degradation of the in situ formed depots. Another two formulations were obtained: formulation F2 containing $4 \mathrm{wt} \% \mathrm{LNG}, 8 \mathrm{wt} \%$ PLGA50:50, 8wt \% PLGA85:15, 8wt\% PLA and $72 \mathrm{wt} \%$ NMP/TEC(90:10); and formulation F3 contained $4 \mathrm{wt} \% \mathrm{LNG}, 4.8 \mathrm{wt} \%$ PLGA50:50, $19.2 \mathrm{wt} \%$ PLA and $72 \mathrm{wt} \%$ 
NMP/TEC (9:1). After subcutaneously injected at LNG dose levels of $46.60 \pm 3.30$ (for $\mathrm{F} 2$ ) and $48.08 \pm 3.39$ (for F3) $\mathrm{mg} / \mathrm{kg}$, the F2 and F3 formulations suppressed the normal ovarian cycles of cottontop tamarins for $120.4 \pm 46.0$ days and $134.8 \pm 32.8$ days, respectively. With the further modification of the formulation F3 including increasing LNG content from $4 \mathrm{wt} \%$ to $6 \mathrm{wt} \%$, reducing the weight percentage of the mixture solvent NMP/TEC (9:1) from $72 \mathrm{wt} \%$ to $70 \mathrm{wt} \%$, a formulation F4 was obtained. After subcutaneously injected at the LNG dose levels of $71.89 \pm 8.90 \mathrm{mg} / \mathrm{kg}$, the F4 formulation extended the suppression of the normal ovarian cycles of cottontop tamarins to 198.8 \pm 70.3 days. However, both the F3 and F4 formulations did show some initial burst. The initial burst release could reduce the API available for the contraceptive, in other words, the release duration could be longer if the initial burst release could be eliminated. Additionally, the polymeric solution formulations had problems with relatively broad duration of the contraception. It has been documented that the initial burst release and/or duration of the API release could be adjusted by varying the polymer/solvent ratio, using a blend of polymers with different end groups, molecular weight, and degradation properties, and adjusting the hydrophobicity/hydrophilicity of the solvents [194, 209]. It has been reported that zero-order release of LNG was achieved in vitro from ISD systems containing PLA (MW 11,000) and a mixture of benzyl benzoate and benzyl alcohol for 3 months with almost no initial burst and less than $40 \%$ of loaded LNG released out after 3 months [210]. Moreover, in order to minimize the initial burst release, the API could also be encapsulated into microparticles and then dispersed/suspended in the ISD systems $[209,211]$. It has been demonstrated that by encapsulating naltrexone base in PLA microparticles prepared via melting/fusion/grounding process, the in situ forming PLGA/NMP depot systems dispersed with these naltrexone/PLA microparticles showed significantly reduced initial burst compared with the PLGA/NMP systems loaded with naltrexone base [209].

\section{Summary}

Overall, the previous efforts to develop polymer-based injectable LARCs have generated a lot of positive data supporting the feasibility of long-acting injectable contraceptives for more than 3 months' contraceptive protection after one intramuscular or subcutaneous injection. However, not many reached the market, and there are not many efforts to continue such investigations with a targeting goal of commercializing the products for contraception. Therefore, there is still an unmet need for affordable injectable LARCs which can provide contraception for more than three months to one year after a single shot so that better adherence and continuation rates can be achieved.

\section{Hypothesis and Specific Aims}

The long-term goal of the project in chapter 2 was to develop injectable dosage forms for sustained release of contraceptives for prevention of unintended pregnancies for six months or longer after a single injection. The immediate objective was to design and develop LNG containing injectable ISD system for contraceptive effect for five 
months after a single injection. The ISD formulation contains the drug LNG, biodegradable polyesters, and a vehicle for injection. LNG is a synthetic levorotatory hormone that is widely used as a contraceptive to prevent unwanted pregnancy in females [212-216], also used as an API for the development of long-acting contraceptives Sinoimplant ${ }^{\circledR}$ and Jadelle $[34,217]$. It has very limited solubility in water $\left(2.05 \mathrm{mg} \cdot \mathrm{L}^{-1}\right)$ which offers to ensure prolonged release. It is superior to medroxyprogesterone regarding bone loss $[1,2]$. The design and development plan for ISD systems is given in Figure 2-3.

The hypothesis of the project in chapter 1, which was the sub-hypothesis 1 of the overall hypothesis of the dissertation, was that injectable in situ depot system with balanced biodegradable polymers and solvents can be developed as an effective delivery system for sustained release of contraceptives such as levonorgestrel for five months or longer after single SubQ injection through a $23 \mathrm{G}$ needle. The specific aims of the project include:

Specific Aim 1: Design and conduct in vitro optimization and characterization of LNG-containing ISD formulations. The ISD formulations containing PLGA, PLA as a polymer matrix, LNG as an API, and NMP and its mixture with TEC, acetyl triethyl citrate (ATEC), benzyl benzoate (BB), benzyl alcohol (BA), ethyl acetate (EA) were prepared. The optimization parameters included polymer type/molecular weight/viscosity/degradation, polymer/solvent ratio, LNG content, injectability, and in vitro acyline release kinetics in PBS ( $\mathrm{pH} 7.4)$ and accelerated conditions.

Specific Aim 2: Evaluate in vivo safety, pharmacokinetics and contraceptive effect of LNG-containing ISD systems in rats. ISD formulations that were injectable through the $23 \mathrm{G}$ needle and shown stable in vitro depot integrity, and long-term in vitro LNG release ( $\geq 3$ months) were injected SubQ into female Sprague-Dawley (SD) rats. Their safety, one day short-term and 6-month long-term pharmacokinetics and contraceptive effects including suppression of estrous cycle were evaluated.

\section{Materials and Methods}

\section{Chemicals and Reagents}

Levonorgestrel (>99.99\%) was provided by Family Heath International (FHI360). Levonorgestrel-D 6 (LNG-D 6 ) was purchased from Hexonsynth (Middle Sex, UK). Esterterminated poly(D, L-lactic-co-glycolic acid) (50:50) with an inherent viscosity (iv) of 0.55-0.75 dL/g was purchased from Lactel Absorbable Polymers (Birmingham, AL). Ester-terminated poly(D, L-lactide) with iv 0.40-0.50 and 0.60-0.80 dL/g were purchased from Lakeshore Biomaterials (Birmingham, AL) and Evonik (Birmingham, AL), respectively. Blank rat plasma was ordered from Bioreclamation IVT, USA. The following chemicals and solvents were obtained from Fisher Scientific (Pittsburgh, PA): TEC, NMP, BB, BA, ATEC, EA, sodium chloride (crystalline/certified ACS), sodium hydrogen phosphate $(98+\%)$, potassium phosphate monobasic (extra pure $99+\%$ ), 


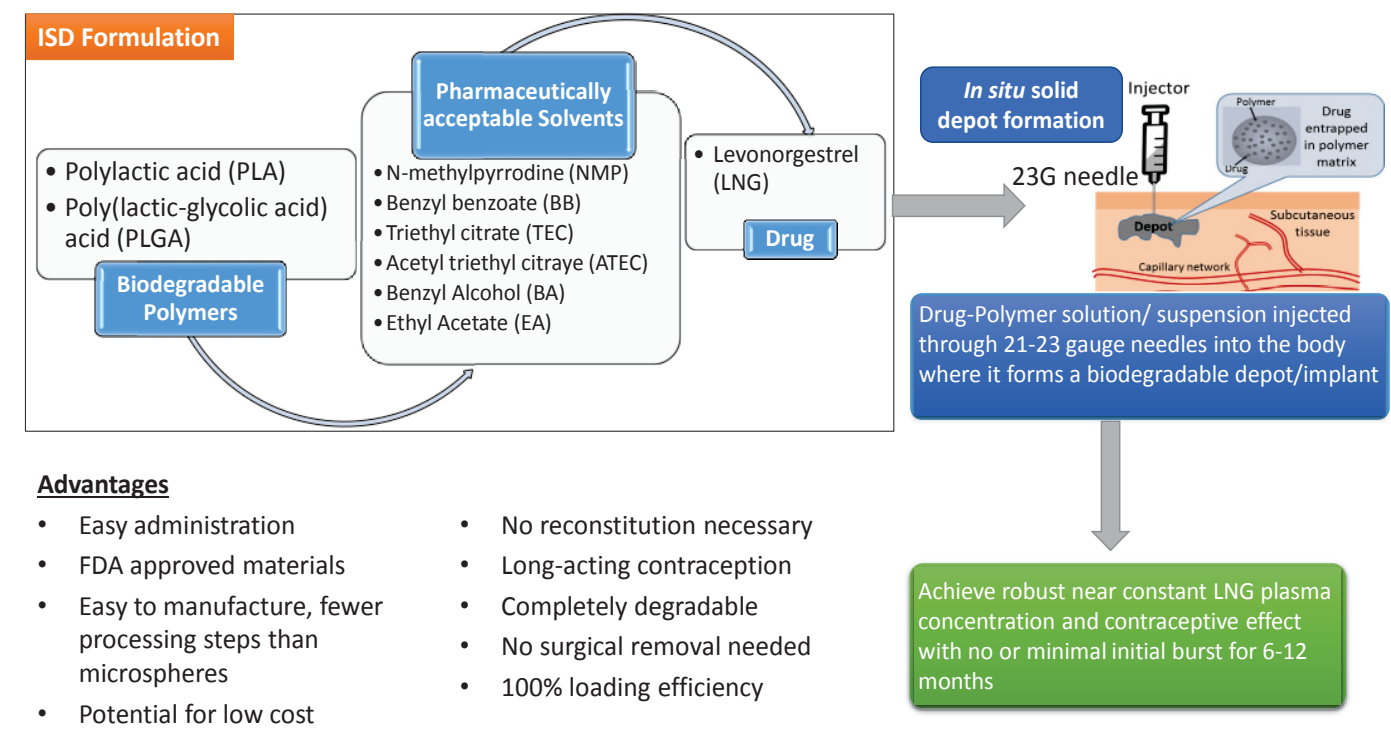

Figure 2-3. Design and developmental plan of injectable ISD dosage forms.

Notes: ISD formulation is a solution or suspension containing LNG and biodegradable polymers (PLA or PLGA) dissolved or dispersed in pharmaceutically acceptable watermiscible organic solvents (NMP, BB, TEC, ATEC, BA, EA), respectively. Upon injection into the tissue, this ISD system forms a solid depot of polymeric matrix at the injection site because of the phase separation (in situ polymer precipitation) by solvent exchange. The LNG entrapped in the depot is then slowly released out into the surrounding body fluid due to the degradation of the polymers and diffusion of the drug, goes into the systemic circulation and then reaches target sites 
potassium chloride, water (HPLC grade), acetonitrile (HPLC grade), methanol (HPLC grade), ethanol (HPLC grade), hexane, formic acid, and Tween 20.

\section{Preparation of Formulations}

The formulations with composition listed in Table 2-2 and Table 2-3 were prepared as follows: LNG was weighed and added to the mixture solvent of NMP/TEC or $\mathrm{NMP} / \mathrm{BB}(9: 1 \mathrm{v} / \mathrm{v})$ in a $20 \mathrm{~mL}$ vial. The vial was vortexed at $500 \mathrm{rpm}$ until LNG was dissolved. Weighed PLGA-50:50 and PLA polymers were then added and vortexed at $500 \mathrm{rpm}$ until a uniform solution was obtained.

\section{In Vitro Evaluation}

\section{Viscosity Measurements}

Shear viscosity of the formulations was measured by using the cone and plate geometry technique by flow procedure on an AR-G2 rheometer, TA Instruments, Waters Corporation, (New Castle, USA), and the results analyzed by TA universal analysis 2000 software. All measurements were performed at $25{ }^{\circ} \mathrm{C}$ with a $20 \mathrm{~mm}$ stainless steel cone with $1^{\circ}$ Cone angle, TA Instruments, Waters Corporation, (New Castle, USA) at a gap of $26 \mu \mathrm{m}$. The sample was allowed to equilibrate for $5 \mathrm{~min}$ at $25^{\circ} \mathrm{C}$ before measurements were conducted. Shear viscosities were measured in the range of shear rate from $0.001-$ $1000 \mathrm{~s}^{-1}$ and then in descending order. The slope of the curve shear stress vs. shear rate was calculated to get the average shear viscosity of the solutions that exhibited plastic flow. The experiments were repeated thrice, and the results were shown as mean $\pm \mathrm{SD}$.

\section{In Vitro Release Study}

\section{Real-time (long-term) in vitro release study}

The formulations were injected $(400 \mu \mathrm{L})$ through a syringe into a PTFE mold having a cylindrical cavity of $12.5 \mathrm{~mm}$ in diameter and $5 \mathrm{~mm}$ in depth. The mold was then transferred into a $500 \mathrm{~mL}$ jar, and $400 \mathrm{~mL}$ PBS (pH 7.4) release medium was carefully added to the jar. The jar was then placed into a $37^{\circ} \mathrm{C}$ shaking incubator (MaxQ800, Thermo Scientific, Waltham, MA) and constantly shaken at $50 \mathrm{rpm}$. At selected time points over a 6-month period, $1 \mathrm{~mL}$ of the release medium was collected for drug analysis using HPLC (see Section 2.3.4 for details), and then the entire medium was replaced with fresh PBS ( $\mathrm{pH}$ 7.4) to maintain the sink condition. Each formulation was run in triplicates for the studies.

\section{Accelerated release study}

The 64 and 96 formulations were selected for conducting a short-term in vitro release study the same way as for the long-term in vitro release study as described 
Table 2-2. The composition of set-1 ISD formulations.

\begin{tabular}{|c|c|c|c|c|c|c|c|}
\hline $\begin{array}{c}\text { Formulation } \\
\#\end{array}$ & $\begin{array}{c}\text { PLGA 50:50 } \\
\text { wt } \% \\
i v-0.63 \\
\end{array}$ & $\begin{array}{c}\text { PLG 85:15 } \\
\text { wt } \% \\
i v-0.63\end{array}$ & $\begin{array}{c}\text { PLA } \\
\text { wt } \% \\
i v-0.25\end{array}$ & $\begin{array}{c}\text { PLA } \\
\text { wt \% } \\
i v-0.47 \\
\end{array}$ & $\begin{array}{c}\text { PLA } \\
\text { wt } \% \\
\text { iv-0.63 }\end{array}$ & $\begin{array}{l}\text { LNG } \\
\text { wt } \%\end{array}$ & $\begin{array}{c}\text { Solvent }(9: 1) \\
\text { wt } \%\end{array}$ \\
\hline 64-Tamrin & 4.7 & & & & 18.8 & 6 & $70.5 \mathrm{NMP} / \mathrm{TEC}$ \\
\hline 55 & & & 12 & & 12 & 4 & $72 \mathrm{NMP} / \mathrm{BB}$ \\
\hline 56 & & & 12 & & 12 & 4 & 72 NMP/BA \\
\hline 57 & & & 12 & & 12 & 4 & 72 NMP/ATEC \\
\hline 61 & & & 12 & & 12 & 4 & 72 NMP/EA \\
\hline 62 & & & 12 & & 12 & 4 & $72 \mathrm{NMP} / \mathrm{TEC}$ \\
\hline 67 & & 12.2 & 12.2 & & & 2.5 & 73.1 NMP/TEC \\
\hline 68 & & 12.2 & 12.2 & & & 2.5 & $73.1 \mathrm{NMP} / \mathrm{BB}$ \\
\hline 96 & 4.1 & & & 20.3 & & 2.5 & $73.1 \mathrm{NMP} / \mathrm{BB}$ \\
\hline 97 & 4.1 & & & 20.3 & & & 73.1 NMP/BB (8:2) \\
\hline 98 & 4.1 & & & 20.3 & & & $73.1 \mathrm{NMP} / \mathrm{BB}(7: 3)$ \\
\hline 99 & 4.1 & & & 20.3 & & & 73.1 NMP/TEC (7:3) \\
\hline 100 & 4.1 & & & 20.3 & & & 73.1 NMP/ATEC (7:3) \\
\hline
\end{tabular}

Notes: Units of inherent viscosity $(i v)$ : $\mathrm{dL}^{-\mathrm{g}^{-1}}$; molecular weights (based on GPC) of polymers according to the certificate of analysis are PLA (iv-0.25): 27 kDa; PLA (iv-0.47): 67 kDa; PLA (iv-0.63): 102 kDa. 
Table 2-3. The composition of set-2 ISD formulations.

\begin{tabular}{|c|c|c|c|c|c|}
\hline Formulation & $\begin{array}{c}\text { PLGA 50:50 } \\
\text { iv-0.63 } \\
\text { wt } \%\end{array}$ & $\begin{array}{c}\text { PLA } \\
\text { iv-0.47 } \\
\text { wt } \%\end{array}$ & $\begin{array}{c}\text { PLA } \\
i v-0.63 \\
\text { wt } \%\end{array}$ & $\begin{array}{l}\text { LNG } \\
\text { wt\% }\end{array}$ & $\begin{array}{c}\text { Solvent }(9: 1) \\
w t \%\end{array}$ \\
\hline 64-Tamrin & 4.7 & & 18.8 & 6 & $70.5 \mathrm{NMP} / \mathrm{TEC}$ \\
\hline 96 & 4.1 & 20.3 & & 2.5 & $73.1 \mathrm{NMP} / \mathrm{BB}$ \\
\hline $96 \mathrm{j}$ & 4 & & 20 & 2.5 & $73.5 \mathrm{NMP} / \mathrm{TEC}$ \\
\hline $96 \mathrm{k}$ & 4 & & 16 & 2.5 & $77.5 \mathrm{NMP} / \mathrm{TEC}$ \\
\hline 961 & 4 & & 12 & 2.5 & $81.5 \mathrm{NMP} / \mathrm{TEC}$ \\
\hline $96 \mathrm{~m}$ & 4 & & 20 & 4 & 72 NMP/TEC \\
\hline $96 n$ & 4 & & 16 & 4 & 76 NMP/TEC \\
\hline 960 & 4 & & 12 & 4 & 80 NMP/TEC \\
\hline $96 p$ & 4 & 4 & 16 & 4 & 72 NMP/TEC \\
\hline $96 q$ & 4 & 8 & 12 & 4 & 72 NMP/TEC \\
\hline $96 r$ & 4 & 16 & 4 & 4 & 72 NMP/TEC \\
\hline $96 z z$ & 4 & 16 & 4 & 4 & 72 NMP/EA \\
\hline
\end{tabular}

Notes: Units of inherent viscosity $(i \mathrm{v}): \mathrm{dL} \cdot \mathrm{g}^{-1}$; molecular weights (based on GPC) of polymers according to the certificate of analysis are PLA (iv-0.25): $27 \mathrm{kDa}$; PLA (iv0.47): $67 \mathrm{kDa}$; PLA (iv-0.63): $102 \mathrm{kDa}$. 
previously in Section 2.3, except for that the release conditions were the four conditions listed in Table 2-4 instead of PBS (pH 7.4) at $37^{\circ} \mathrm{C}$ and the study was over a two week period instead of long-time period (conditions designed by Dr. Wang). The correlations between the release kinetics and mechanisms of LNG from ISD systems (64 and 96) under real-time and accelerated conditions were established. The best-correlated condition was used to conduct the short-term $(24 \mathrm{~h})$ accelerated release study during the screening of the set-2 formulations.

\section{HPLC Measurements}

The collected release medium samples were analyzed for LNG content using HPLC (Shimadzu Scientific Instruments, Inc. Columbia, MD) installed with a reverse phase column BDS Hypersil C- $18,3 \mu \mathrm{m}, 100 \times 4.6 \mathrm{~mm}$. Acetonitrile (ACN) $/ 0.01 \%$ formic acid in water $(65: 35 \mathrm{v} / \mathrm{v})$ was used as a mobile phase. The flow rate was set at 0.8 $\mathrm{mL} \cdot \mathrm{min}^{-1}$, and the detection wavelength was $245 \mathrm{~nm}$. The injection volume was $50 \mu \mathrm{L}$. The column temperature was kept at $25^{\circ} \mathrm{C}$. The standards for calibration were 0.070 , $0.100,0.250,0.500,0.750,1.00,2.50 \mu \mathrm{g} \cdot \mathrm{mL}^{-1}$ of LNG dissolved in PBS (pH 7.4 correlation coefficient was $R^{2}>0.99$ ). The cumulative release amount of LNG was calculated from the LNG concentrations determined in the samples.

\section{In Vivo Evaluation}

\section{LC-MS/MS Method Development and Validation for Analysis of LNG in Rat Plasma}

Liquid chromatography (LC) was performed on a UPLC system (Shimadzu Corporation) consisting of a binary pump, column oven, and degasser units. $10 \mu \mathrm{L}$ of sample extracts were injected into the reversed-phase Luna C18 (2) column (50 x $2.0 \mathrm{~mm}$ i.d., $3 \mu \mathrm{M}$; Phenomenex, Torrance, CA, USA) by using an autosampler (Eksigent, part of AB SCIEX). Acetonitrile/water mixture $(70 / 30, \mathrm{v} / \mathrm{v})$ containing $0.01 \%$ formic acid was used as a mobile phase and was pumped at a flow rate of $0.3 \mathrm{~mL} \cdot \mathrm{min}^{-1}$. Total run time was 3 min for each sample injection. The column temperature was kept at $25^{\circ} \mathrm{C}$. The LC elute was introduced into the Applied Biosystems Triple Quad ${ }^{\mathrm{TM}} 4500$, a triplequadrupole tandem mass spectrometer equipped with turbo spray ionization source, for quantification of the analytes in positive ion mode (ESI+). Detection of the analyte ions was performed in a multiple reaction monitoring mode and transitions of $\mathrm{m} / \mathrm{z}$ $313.10 \rightarrow 245.10$, and $319.10 \rightarrow 251.10$ were used for LNG and LNG-D 6 , respectively. Analyst ${ }^{\circledR}$ 1.6.2 software was used for processing the data. The detailed experimental conditions are: ion spray voltage $4500 \mathrm{v}$, collision gas 8 psi, temperature $500{ }^{\circ} \mathrm{C}$, collision energy $23 \mathrm{v}$, entrance potential $10 \mathrm{v}$, collision exit potential $12 \mathrm{v}$, declustering potential $81 \mathrm{v}$, curtain gas $40 \mathrm{psi}$, ion spray gas I 60 psi and ion spray gas II 60 psi 
Table 2-4. Release testing conditions used for accelerated in vitro release method development.

\begin{tabular}{cccccc}
\hline Condition & $\begin{array}{c}\text { PBS } \\
(\% \mathbf{\%} / \mathbf{v})\end{array}$ & $\begin{array}{c}\text { Ethanol } \\
(\% \mathbf{\%} / \mathbf{v})\end{array}$ & $\begin{array}{c}\text { Tween 20 } \\
(\% \mathbf{\%} / \mathbf{v})\end{array}$ & $\begin{array}{c}\mathbf{p H} \\
\text { Temperature } \\
\left({ }^{\circ} \mathbf{C}\right)\end{array}$ \\
\hline A & $100 \%$ & 0 & 0 & 7.4 & 50 \\
B & Adjusted to $100 \%$ & $25 \%$ & 0 & 7.4 & 50 \\
C & Adjusted to $100 \%$ & $25 \%$ & $0.5 \%$ & 7.4 & 50 \\
D & Adjusted to $100 \%$ & $25 \%$ & $0.5 \%$ & 9.0 & 50 \\
\hline
\end{tabular}




\section{Preparation of standard and quality control samples}

For the LNG calibration standard solution preparation, LNG was accurately weighed and dissolved in the mixture of methanol and water $(50 / 50, \mathrm{v} / \mathrm{v})$ to obtain a stock solution with a concentration of $1 \mathrm{mg} \cdot \mathrm{mL}^{-1}$. This stock solution was then diluted with methanol/water $(50: 50, \mathrm{v} / \mathrm{v})$ mixture to obtain working stock solutions at concentrations $5,10,25,50,100,250$ and $500 \mathrm{ng} \cdot \mathrm{mL}^{-1}$. Furthermore, these working stock solutions were diluted 10 times with blank rat plasma to obtain working standard solutions at concentrations $0.5,1,2.5,5,10,25$ and $50 \mathrm{ng} \cdot \mathrm{mL}^{-1}$.Quality control samples for $\mathrm{LNG}$ were also prepared from a separate set of working stock solutions as mentioned above at three concentrations 0.5 (low; LLOQ), 5 (medium; MLOQ) and 50 (high; HLOQ) $\mathrm{ng} \cdot \mathrm{mL}^{-}$ ${ }^{1}$. Being the structural analog of LNG, LNG-D 6 was used as an internal standard (IS). A stock solution of LNG-D 6 was prepared in a mixture of methanol and water $(50: 50, \mathrm{v} / \mathrm{v})$ to obtain the final concentration of $250 \mu \mathrm{g} \cdot \mathrm{mL}^{-1}$. All the stock solutions were stored at $20^{\circ} \mathrm{C}$ and were brought to room temperature before use.

\section{Extraction procedure}

$100 \mu \mathrm{L}$ of rat plasma containing $\mathrm{LNG}$ and $\mathrm{LNG}-\mathrm{D}_{6}$ was taken in a microcentrifuge tube, and $500 \mu \mathrm{L}$ of hexane/ethyl acetate $(80 / 20, \mathrm{v} / \mathrm{v})$ mixture was added followed by vortexing for $3 \mathrm{~min}$. The tubes were centrifuged at $10,000 \mathrm{x}$ g at $4{ }^{\circ} \mathrm{C}$ for 10 min. Supernatant organic phase containing the LNG and LNG-D 6 was collected into another microcentrifuge tube and subjected to vacuum vaporization. The resultant residue was dissolved in $50 \mu \mathrm{L}$ of reconstitution solvent methanol/water $(50 / 50, \mathrm{v} / \mathrm{v})$ mixture and vortexed for $2 \mathrm{~min}$ for proper mixing. In this process, $100 \mu \mathrm{L}$ of rat plasma was concentrated to $50 \mu \mathrm{L}$ resulting in increased sensitivity. From the extracted sample, 10 $\mu \mathrm{L}$ was injected into the $\mathrm{LC} / \mathrm{MS} / \mathrm{MS}$ system for analysis.

\section{Matrix effect}

Matrix effect is the effect of rat plasma constituents on the ionization of LNG and LNG- $\mathrm{D}_{6}$. Due to the endogenous compounds from rat plasma, the ionization of an analyte can be suppressed or increased. It is determined by comparing the analyte peaks obtained from samples spiked in the solution of the rat plasma extracted by hexane/ethyl acetate $(80 / 20, \mathrm{v} / \mathrm{v})$ mixture(post-extracted spiked samples) with those spiked in mobile phase acetonitrile/water mixture $(70 / 30, \mathrm{v} / \mathrm{v})$ containing $0.01 \%$ formic acid (neat standard samples). Matrix effect was calculated at LLOQ $\left(0.5 \mathrm{ng} \cdot \mathrm{mL}^{-1}\right)$, MLOQ $\left(5 \mathrm{ng} \cdot \mathrm{mL}^{-1}\right)$ and HLOQ $\left(50 \mathrm{ng} \cdot \mathrm{mL}^{-1}\right.$ ) concentrations $(\mathrm{n}=6)$ for $\mathrm{LNG}$ and at $25 \mathrm{ng} \cdot \mathrm{mL}^{-1}$ (working concentration) for LNG-D $_{6}$, using the Equation 2-1 listed below.

$$
\% \mathrm{ME}=\frac{\text { Peak Area of Post-extracted Standard Sample }}{\text { Peak Area of Neat Standard Sample }} * 100
$$

\section{Specificity and sensitivity}

Blank plasma, and analyte LNG (LLOQ) and internal standard LNG- $\mathrm{D}_{6}$ spiked plasma samples were treated according to the extraction procedure mentioned above prior 
to analysis by the LC/MS/MS. The chromatograms for the blank plasma and spiked plasma samples were compared to identify any peaks during the analysis. Specificity was evaluated by checking any significant interference in the chromatograms at the peak regions of LNG and LNG- $\mathrm{D}_{6}$. The LLOQ and Limit of detection (LOD) were defined at the lowest concentrations that resulted in a signal to noise $(\mathrm{S} / \mathrm{N})$ ratio of 10 and 3 , respectively.

\section{Recovery efficiency}

The recovery efficiency $(\% \mathrm{RE})$ for the analyte $\mathrm{LNG}$ and internal standard LNG$\mathrm{D}_{6}$ was calculated by comparing the peak areas of the LNG/LNG- $\mathrm{D}_{6}$ added into blank plasma and extracted using the extraction mixture solvent hexane/ethyl acetate (80/20, $\mathrm{v} / \mathrm{v}$ ) with those of LNG spiked directly into the extraction mixture solvent (post-extracted standard samples) at the three LNG QC $\left(0.5,5\right.$ and $\left.50 \mathrm{ng} \cdot \mathrm{mL}^{-1}\right)$ and one LNG- D6 working $\left(25 \mathrm{ng} \cdot \mathrm{mL}^{-1}\right)$ concentrations, using Equation 2-2 listed below:

$$
\% \mathrm{RE}=\frac{\text { Peak Area of Extracted Sample }}{\text { Peak Area of Post-extracted Standard Sample }} * 100
$$

\section{Linearity}

Seven calibration standard concentrations in the range of $0.5-50 \mathrm{ng} \cdot \mathrm{mL}^{-1}(0.5,1$, 2.5, 5, 10, 25 and $50 \mathrm{ng} \cdot \mathrm{mL}^{-1}$ ) were extracted and analyzed for drawing the calibration curve. The peak areas were calculated, and peak area ratios (Analyte/IS) were fitted to linear regression analysis using 1/x2 as a weighing factor. The concentrations of the samples were back calculated from the standard curve and were compared to deviation from the theoretical concentrations. The deviations should be within $\pm 20 \%$ and $\pm 15 \%$ for the LLOQ and other concentrations, respectively. The correlation coefficient ( $\mathrm{r} 2)$ for the calibration curve should be 0.99 or higher.

\section{Accuracy and precision}

Accuracy was determined as the percentage of the difference between the mean calculated concentration and the theoretical value, as shown in Equation 2-3 below:

$$
\% \text { Accuracy }=\left[1-\frac{(\text { Actual-Measured) Concentration }}{\text { ActualConcentration }}\right] * 100
$$

Precision indicates the reproducibility and measures the closeness among the replicates. It was determined as the percentage of the standard deviation from the arithmetic mean of the calculated sample concentrations and expressed as the percent of the coefficient of variation $(\% \mathrm{CV})$, as shown in Equation 2-4 below:

$$
\% \mathrm{CV}=\left(\frac{\text { Standard Deviation }}{\text { Mean Concentration }}\right) * 100
$$




\section{Intra-day assay accuracy and precision}

Seven calibration standard samples with concentrations in the range of $0.5-50$ $\mathrm{ng} \cdot \mathrm{mL}^{-1}\left(0.5,1,2.5,5,10,25\right.$ and $\left.50 \mathrm{ng} \cdot \mathrm{mL}^{-1}\right)$ and three QC samples, LLOQ $\left(0.5 \mathrm{ng} \cdot \mathrm{mL}^{-}\right.$ $\left.{ }^{1}\right)$, MLQC $\left(5 \mathrm{ng} \cdot \mathrm{mL}^{-1}\right)$ and ULOQ $\left(50 \mathrm{ng} \cdot \mathrm{mL}^{-1}\right)$ were prepared separately in 6 replicates, and analyzed for accuracy and precision measurements using chromatography for 3 consecutive days. The accuracy should be within $\pm 20 \%$ and $\pm 15 \%$ forthe LLOQ and all the other concentrations, respectively. The $\% \mathrm{CV}$ should not exceed $20 \%$ and $15 \%$ for the LLOQ and all the other concentrations, respectively.

\section{Inter-day assay accuracy and precision}

The data obtained above for the intra-day assay accuracy and precision were reanalyzed for inter-day assay accuracy and precision using all three days' data for each concentration (18 samples in total), and the results were reported. Both the accuracy and $\% \mathrm{CV}$ should be within $\pm 20 \%$ and $\pm 15 \%$ forthe LLOQ and all the other concentrations, respectively.

\section{Stability}

Stability of the LNG in the rat plasma and final reconstitution solvent under specific experimental conditions was evaluated. Three QC samples with concentrations in the rat plasma, 0.5 (low), 5 (medium) and $50 \mathrm{ng} \cdot \mathrm{mL}^{-1}$ (high) were prepared in 6 replicates for these studies. Long-term stability of LNG was investigated by measuring the accuracy after the QC samples were stored at $-80{ }^{\circ} \mathrm{C}$ (storage condition) for 15 days and under 5 cycles of the freeze-thaw process from $-80{ }^{\circ} \mathrm{C}$ to room temperature. In process stability was tested after the QC samples were kept in the rat plasma at room temperature for $4 \mathrm{~h}$. Post-operative stability was studied after the final processed samples were kept in the reconstitution solvent methanol/water $(50 / 50, \mathrm{v} / \mathrm{v})$ mixture at room temperature for $8 \mathrm{~h}$ at $10{ }^{\circ} \mathrm{C}$ (autosampler injector temperature) for $24 \mathrm{~h}$. LNG was considered to be stable if the percent change in concentration was within $\pm 15 \%$.

\section{LNG pharmacokinetics in rat}

To evaluate the applicability of the developed method, the SubQ pharmacokinetic profile of LNG in rats was determined. 2.5 month old female Sprague Dawley rats $(n=6)$ were ordered and housed in plastic cages at controlled temperature $\left(24 \pm 0.5^{\circ} \mathrm{C}\right)$ and light controlled environment (12h light/12h dark). Rats were provided with normal rat chow and water ad libitum. All the experiments were approved by the University of Tennessee Institutional Animal Care and Use Committee (IACUC). Before the study was conducted, rats were anesthetized by isoflurane inhalation and $4 \mathrm{mg}$ of LNG in $400 \mu \mathrm{LNMP}$ solution was injected subcutaneously into each rat. 200-250 $\mu \mathrm{L}$ of blood samples were collected from the experimental rats and put into Li-heparin coated blood collection tubes at 0.25 , $0.5,1,4,8,24$, and $48 \mathrm{~h}$ time points post the injection. The tubes were centrifuged at a speed of $12,000 \mathrm{~g}$ for $10 \mathrm{~min}$, and the obtained plasma samples were stored at $-80{ }^{\circ} \mathrm{C}$ for further analysis. LNG was extracted from the plasma samples using hexane and ethyl 
acetate solvent mixture as described in the above sections. The resulting solutions were diluted to the concentrations that fell in the standard curve concentration range. The pharmacokinetic parameters were calculated by non-compartmental analysis using Phoenix WinNonlin 6.3 (Certara, NJ).

\section{In Vivo Pharmacokinetic Study of Formulations}

All the rats were ordered from Harlan Laboratories (Indianapolis, IN, USA), and housed in metabolic cages under controlled temperature $\left(24 \pm 0.5^{\circ} \mathrm{C}\right)$ and light environment ( $12 \mathrm{~h}$ light $/ 12 \mathrm{~h}$ dark). They were permitted to move freely and provided with normal rat chow and water ad libitum. All the experiments were approved by the University of Tennessee Institutional Animal Care and Use Committee (IACUC). Female SD rats ( 2.5 month old) with a heparin-coated polyethylene catheter inserted into each rat's right jugular vein were used to determine the initial burst concentrations of LNG in the plasma ( $24 \mathrm{~h}$ study). Formulations were prepared and exposed to UV light in cell culture hood for 1 hour, and the desired amount of formulation was transferred into sterilized syringe with a $21 \mathrm{G} / 23 \mathrm{G}$ needle. Before the study was conducted the rats were anesthetized by isoflurane inhalation and the selected formulations were injected subcutaneously into each rat. About $0.05-0.1 \mathrm{~mL}$ of blood was withdrawn through the indwelling catheter to clear the heparin in the line. Afterwards, another $0.20-0.25 \mathrm{~mL}$ of blood sample was collected through the catheter and put into Li-heparin coated microcentrifuge tubes at $0.25,0.5,1,4,8$, and $24 \mathrm{~h}$ time points post the injection. After each blood sampling, the catheter was flushed with $0.25-0.5 \mathrm{~mL}$ of heparinized saline solution to prevent blood coagulation inside the catheter. Periodically the rats were supplemented saline to replace the fluid volume.

Long-term studies were carried out on normal female SD rats. The rats were measured for body weight and anesthetized by isoflurane inhalation, and the formulation was then subcutaneously injected into rats at the flank place at a dose of $10 \mathrm{mg}$ LNG. At pre-set time points, body weights of the rats were measured and about $0.2-0.3 \mathrm{ml}$ blood was withdrawn from the tail vein of the rats under anesthesia. All blood samples were placed in Li-heparin coated micro-centrifuge tubes. The plasma samples were obtained by centrifuging the blood-containing micro-centrifuge tubes at a speed of $12,000 \times \mathrm{g}$ for 10 min and then stored at $-80^{\circ} \mathrm{C}$ until analysis. All plasma LNG levels were analyzed according to the LC-MS/MS method described earlier.

\section{Vaginal Cytology}

Periodically the rats were examined for estrous cycle progression by vaginal cytology. At intermittent pre-set weeks, vaginal smears were taken from each rat in the groups in the morning at approximately the same time of the day to minimize the incidence of transitional and missed stages. Cells from the vaginal cell lining were collected by flushing the vagina by introducing $0.3-0.4 \mathrm{~mL}$ of normal saline into the opening of the rat vagina using eye droppers and gently aspirating the content for four times with the same drop of saline to collect more cells. The resulting cell suspension is placed on a clean slide fixed and stained with a Diff-Quick solution and observed 
microscopically. The reproductive cycle (estrous cycle) of female rats is characterized as proestrus, estrus, metestrus and diestrus. Three types of cells could be recognized: round and nucleated ones are epithelial cells; irregular ones without nucleus are the cornified cells, and the little round ones are the leukocytes. The proportion of them was used for the determination of the estrous cycle phases [218].

\section{Fertility Study}

Female rat fertility study was conducted after the end of the treatment (when the drug levels were stopped detecting in vivo). The rats were housed in standard cages grouped as two female rats and one male rat in each cage. Vaginal smears were collected and evaluated for the stage of estrous. At the time of insemination, females were separated from the male rats, and body weights were measured every alternative day (This study was conducted by our lab member Dr. Ananthula).

\section{Results and Discussion}

\section{Screening of Set-1 ISD Formulations}

Although long-acting ISD systems have proven to be effective, the major limitations associated with these systems are injectability and high initial burst [194]. Most of these ISD systems are high viscous fluids and need a lower (thicker) gauge needle (such as 18G/19G) for injection, which will be painful and limits the patient compliance [194]. Attempts made to lower the viscosity of the fluids would usually result in an increase in the initial burst and may also compromise the long-term drug release. A high initial burst would lead to a loss of a major percentage of the drug during initial few hours' post injection and result in a smaller proportion of the total drug load left in the system/depot for long-term release. This high initial release may also lead to toxic levels of the drug in the plasma.

Therefore, the three key attributes that were considered during the formulation optimization are:

- Low viscosity so that it can be injected through $23 \mathrm{G}$ needle

- Low initial burst

- Long-term release profile for at least 5 months

Previously an ISD formulation (\# 64) was used to evaluate the feasibility of using LNG as an effective contraception option for cottontop tamarins, and the results demonstrated that subcutaneous LNG depot injection (64 formulation) was an effective, reversible contraceptive option for the management of cotton-top tamarins [197]. 64 formulation has shown promising potential by demonstrating long-term in vivo suppression of ovarian cycle in cottontop tamarins for 6 months. However, the limitation associated with this formulation is its high viscosity which makes it difficult to inject 
through $23 \mathrm{G}$ needle. Therefore, formulation 64 was chosen as the base ISD formulation on which further design and optimization were carried out to achieve an ideal and robust lead formulation that would satisfy the requirements of injectability, initial burst, and long-term release profile. In the earlier study, LNG was loaded into 64 formulation and directly injected in vivo in cottontop tamarins, and the drug levels were detected only in the feces [197]. Thus, no in vitro characterization data was available for 64 formulation in terms of viscosity, injectability, in vitro release, and in vivo initial burst, and long-term profiles of the drug levels in the plasma. Therefore, 64 formulation was selected to characterize further in this study along with other formulations for the viscosity, injectability and in vivo profile in rats to get a better understanding of the formulation and guide the optimization and development process.

In this project, to addresses some of the limitations of the ISD systems such as injectability, and initial burst in a unique and promising way, an initial set of formulations (set-1) were designed using a rationale approach of incorporating PLA alone or combining a blend of PLGA/PLA with different inherent viscosities, using solvent combination NMP and other hydrophobic co-solvents, and varying the ratios of polymer, as well as the solvent for dissolving the LNG, and varying drug ratios (Table 2-2). The PLGA polymer concentration used in Atrigel ${ }^{\circledR}$ based marketed ISD formulations (Eligard ${ }^{\circledR}$ ) is in the range of 33-44 wt\% (in NMP), based on therapeutic duration (1-6 months), but such a high polymer percentage makes them difficult to be injected through a thinner needle (21-23 G). To maintain the viscosity low, the polymer concentration during the study was adjusted around $24 \mathrm{wt} \%$. However, lowering the polymer percentage in ISD formulations would usually result in high burst and reduced therapeutic duration. Therefore, to compensate this and achieve low initial burst and extended release profile, a hydrophobic polymer PLA with a high inherent viscosity (or MW) is incorporated in the preparations either alone or in combination with PLGA 50:50 or 85:15. Because of its hydrophobic nature, PLA degrades at a slower rate and absorbs less water than PLGA [15]. Among set-1 formulations (Table 2-2) that were designed: one subset of formulations $(55,56,57,61$ and 62) exclusively contained PLA as the polymer, while the polymer composition in another subset $(96,97,98,99,100)$ contained a blend of PLA and PLGA. Mixing two polymers of different MWs permits the control of the timing associated with degradation release [219]. NMP is used as the primary solvent (at least $70 \%$ of the solvent) in the formulations because of its low viscosity, established safety profile, and use in commercial products for human use [181, 190]. Hydrophobic solvents such as TEC, BB, BA, ATEC are used as co-solvent in the formulations, as it was reported in the literature that the low affinity (hydrophobic) solvents in the polymer solutions would slow down the solidification rate and increase the chance for a more uniform release over an extended time period, lower initial burst, and uniformly dense depot morphology with a smaller pore size $[181,194]$. However, the hydrophobic solvents such as TEC (35.2 cP) and BB ( $8.3 \mathrm{cP})$ are viscous solvents than NMP $(1.65 \mathrm{cP})$ and increasing the percentage of co-solvent (e.g., TEC, BB) would increase the viscosity of the formulations, therefore the levels were adjusted to $10-30 \mathrm{wt} \%$ of the solvent. The drug loading was lowered from $6 \%$ to $2.5-4 \%$ to avoid any potential drug solubility issues with long-term storage. The initial screening of the set-1 formulations for the manual injectability has shown that all formulations can be injected through $23 \mathrm{G}$ needle, 
while 64 formulation can only be injected through $21 \mathrm{G}$ needle. The formulations that contained higher proportions of co-solvent (20 and 30\%) showed slight difficulty compared to the formulations that contained $10 \%$ of the co-solvent.

\section{In Vitro Release of ISD Formulations Set-1}

The majority of in vitro drug release studies for the ISD systems have been conducted using variants of the sample-and-separate methodology. The studies were conducted by either injecting the formulation directly into a release medium or dialysis membrane which is then placed in a release medium, or holding the pre-formed gel or depot in a retainer including dialysis membrane [12]. However, the method of injecting the ISD formulation directly into the release medium usually generate depots of irregular shapes, which in turn results in unpredictable in vitro drug release. The dialysis method is dependent on the permeation of a drug across the dialysis membrane [32] and sometimes does not work for the release of certain drugs. Therefore, to avoid the potential issues mentioned above, in this study a simple and inexpensive in vitro release technique was developed for the screening of the formulations using a cylindrical mold made of polytetrafluoroethylene (PTFE) with defined geometry $(2.5 \mathrm{~mm}$ in diameter and $5 \mathrm{~mm}$ in depth) to study in vitro drug release kinetics from injectable ISD systems. The formulations were injected into the mold and then immersed in the release medium in a glass jar (Figure 2-4), and the drug would diffuse out from the top surface into the release medium in a controlled manner. Though this method might not mimic well the in vivo drug release profile, it was sufficient and effective for achieving the objective of this study which is to screen formulations at early development stage.

The cumulative percentage release of LNG from the set-1 ISD formulations for two months is given in Figure 2-5. Apart from 64 formulation, two formulations $(55,96)$ were selected (one from each subset) to continue the long-term release study. The rationale for choosing these two formulations was that they had shown slowest LNG release profile in vitro, and favorable injectability compared to the other formulations. As 55 formulation contained only PLA while 96 formulation has contained a blend of PLA and PLGA, selection of these formulations also help to investigate the effect of the polymer composition (i.e. using either PLA alone or a blend of PLA/PLGA) on long term release. The composition of the three formulations $(55,64$ and 96) along with their viscosity and injectability values is given in Table 2-5. Viscosity data has demonstrated that 55 and 96 formulations have shear viscosities $0.64 \pm 0.04 \mathrm{~Pa}$.s and $0.67 \pm 0.05 \mathrm{~Pa} . \mathrm{s}$, respectively, and can be injected through $23 \mathrm{G}$ needle, while the 64 formulation has a higher shear viscosity of $1.71 \pm 0.01 \mathrm{~Pa} . \mathrm{s}$ and shown injectability through $21 \mathrm{G}$ needle. Even though the overall polymer percentage is similar in all three formulations, the higher viscosity of 64 could be attributed to the differences in terms of drug concentration or loading ( $6 \%$ vs $4 \% / 2,5 \%)$, PLA polymer inherent viscosity ( 0.63 vs 0.47 ), and cosolvent (TEC vs BB). However, the primary factor that resulted in an increased viscosity of the 64 formulation could be the higher inherent viscosity (high MW) of the polymer (PLA - 0.63 i.v.) used in the formulation. Figure 2-6 shows the long-term percent cumulative in vitro LNG release and release rate from the ISD formulations 55, 64, and 96 at $37^{\circ} \mathrm{C}$ in PBS (pH 7.4). 


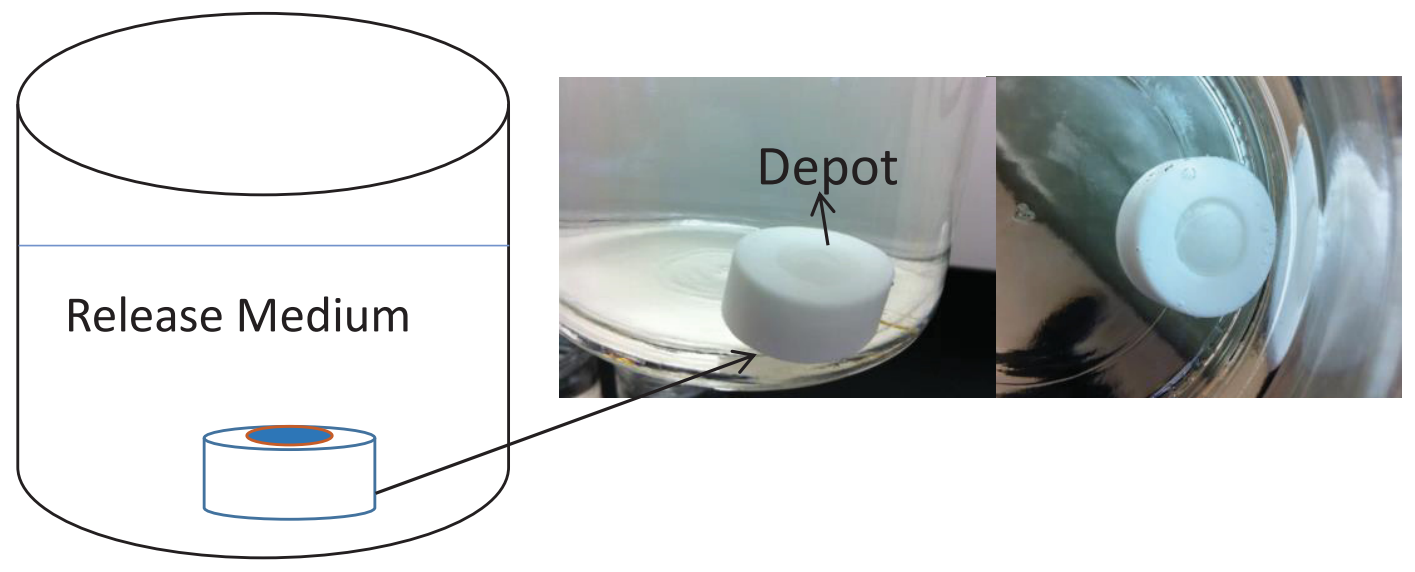

Figure 2-4. Teflon mold holding the depot for in vitro LNG release studies.

Notes: The ISD formulations were injected into the Teflon mold $(2.5 \mathrm{~mm}$ in diameter and $5 \mathrm{~mm}$ in depth) and then immersed in the jar containing release medium for in vitro release studies. At selected time points the release medium was sampled, and complete medium was replaced with fresh medium to maintain sink conditions 


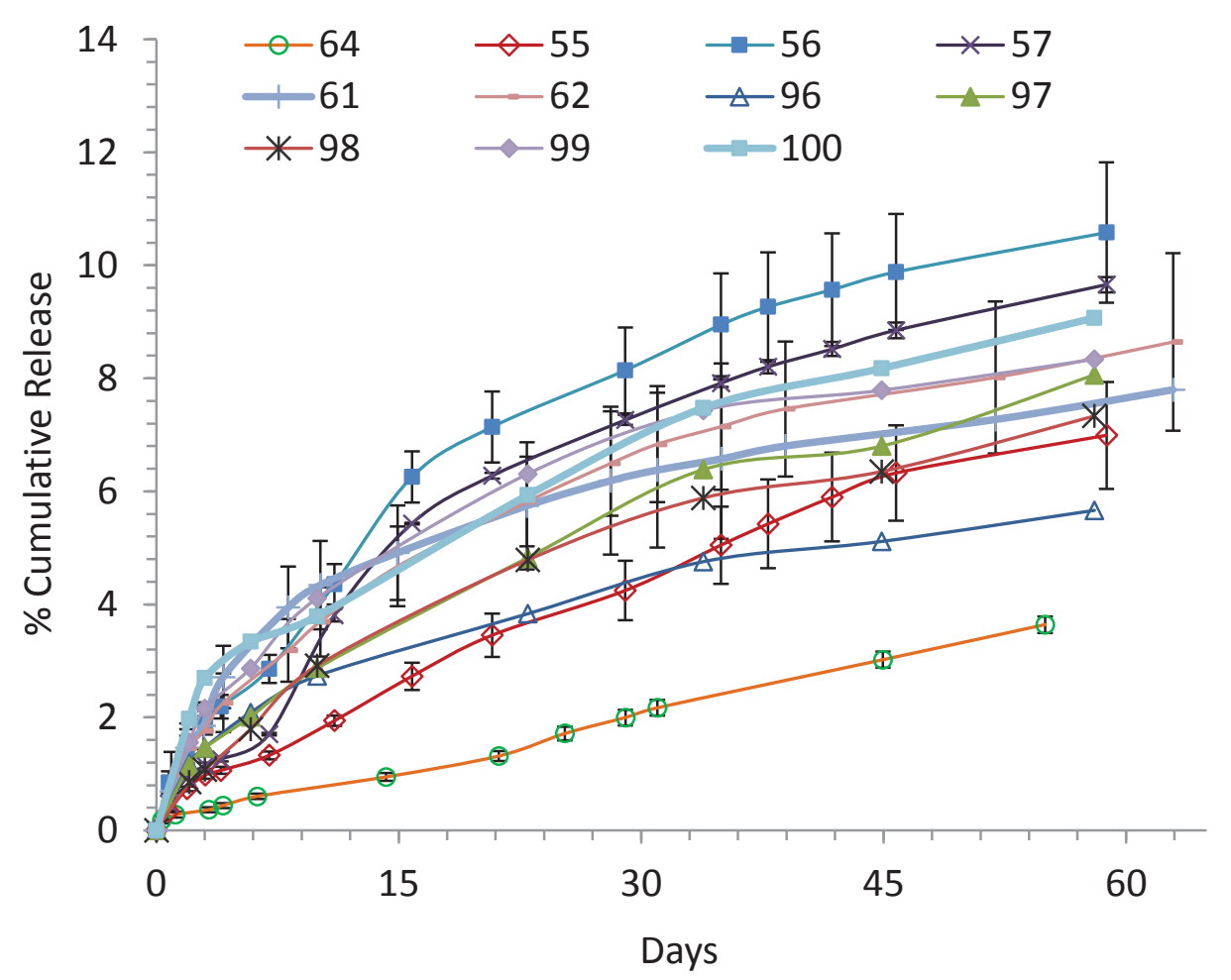

Figure 2-5. In vitro cumulative LNG release percentage from set-1 ISD formulations.

Notes: The set-1 formulations (Table 2-2) were tested for in vitro release for a period of about 2-months. 55 (open diamond), 64 (open circle), and 96 (open triangle) formulations showed slowest in vitro profile and were selected for long-term evaluation. 
Table 2-5. The composition and viscosity of the 55, 64 and 96 formulations.

\begin{tabular}{ccccccccc}
\hline F\# & $\begin{array}{c}\text { PLGA50:50 } \\
\text { (iv-0.63) }\end{array}$ & $\begin{array}{c}\text { PLA } \\
\text { (iv-0.25) } \\
\text { wt\% }\end{array}$ & $\begin{array}{c}\text { PL\% } \\
\text { (iv-0.47) }\end{array}$ & $\begin{array}{c}\text { PLA } \\
\text { wt\% }\end{array}$ & $\begin{array}{c}\text { LNG } \\
\text { (iv-0.63) } \\
\mathbf{w t} \%\end{array}$ & $\begin{array}{c}\text { Solvent (9:1) } \\
\text { wt\% }\end{array}$ & $\begin{array}{c}\text { Shear } \\
\text { viscosity } \\
\text { Pa.s }\end{array}$ & $\begin{array}{c}\text { Injectability } \\
\text { gauge }\end{array}$ \\
\hline 55 & & 12 & 12 & & 4 & $73.1 \mathrm{NMP} / \mathrm{BB}$ & $0.64 \pm 0.04$ & 23 \\
64 & 4.7 & & & 18.8 & 6 & $70.5 \mathrm{NMP} / \mathrm{TEC}$ & $1.71 \pm 0.01$ & 21 \\
96 & 4.1 & & 20.3 & & 2.5 & $73.1 \mathrm{NMP} / \mathrm{BB}$ & $0.67 \pm 0.05$ & 23 \\
\hline
\end{tabular}

Notes: Units of inherent viscosity $(i v): \mathrm{dL} \cdot \mathrm{g}^{-1}$; molecular weights (based on GPC) of polymers according to the certificate of analysis are PLA (iv-0.25): 27 kDa; PLA (iv-0.47): 67 kDa; PLA (iv-0.63): 102 kDa. 

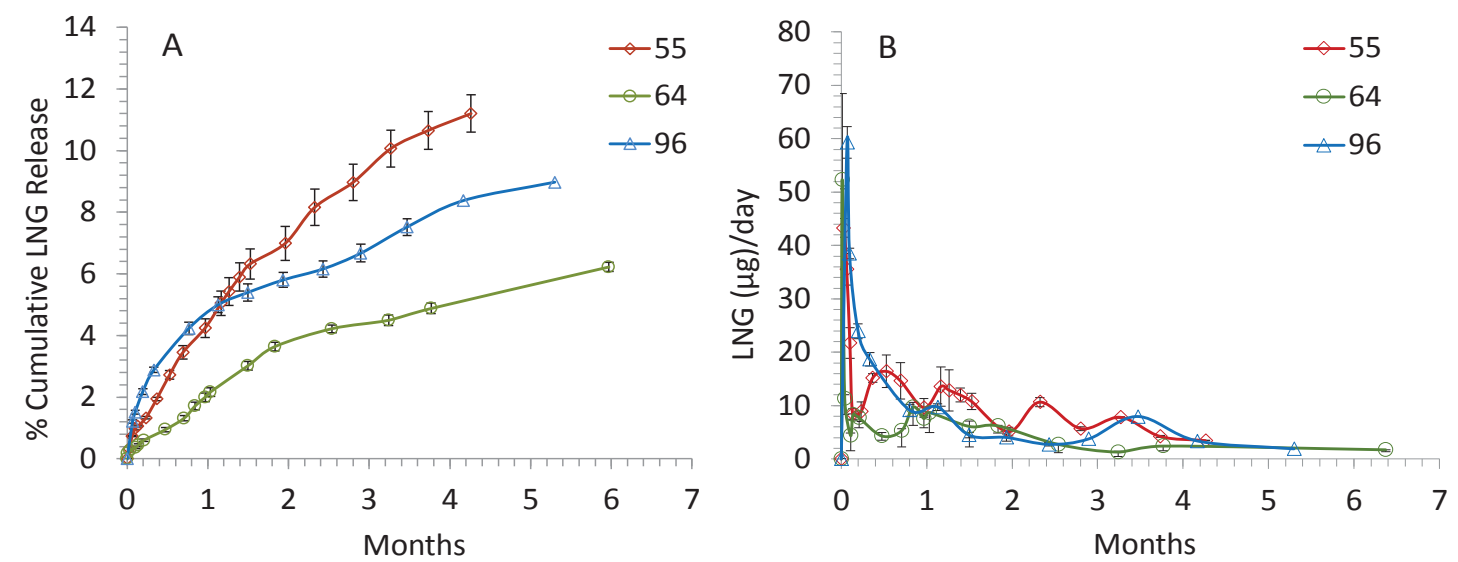

Figure 2-6. In vitro cumulative LNG release (A), and in vitro LNG release amount per day (B) from the ISD formulations.

Notes: The release of LNG from 55 (diamond), 64 (circle) and 96 (triangle) ISD formulations in PBS medium $\left(\mathrm{pH} \mathrm{7.4)}\right.$ at $37^{\circ} \mathrm{C}$. The complete composition of the formulations listed in Table 2-2. Each point represents the mean $\pm \mathrm{SE}, \mathrm{n}=3$. 
The in vitro release of LNG from ISD formulations showed a slight initial burst phase followed by near zero order release. The burst effect in the initial phase of the release is commonly observed with ISD formulations and is dependent on the phase inversion dynamics during the solidification of the matrix, as well as the drug-polymer interaction [184, 220]. Following this initial phase, the release of LNG was slow, and less than $10 \%$ of LNG release from the depot formulations was observed over a 6-month time period. The observed initial burst and the overall percent cumulative LNG release at the end of five months in vitro release were low, and this can be attributed to the release model used in this study where the drug release from the Teflon mold is only limited to the single surface. When the formulations are in the near zero order release stage, 55, 64 and 96 released about 4-18 $\mu \mathrm{g}, 2-9 \mu \mathrm{g}$, and 3-18 $\mu \mathrm{g}$ LNG per day, respectively, after dose normalization. The differences in the release profile could be attributed to the differences in the composition of the formulations regarding the polymer type, inherent viscosity of the PLA polymer, drug loading, and co-solvent. 55 and 96 formulations, which have a greater percentage of smaller inherent viscosity PLA polymers, showed faster release profile compared to the 64 formulation which has higher inherent viscosity PLA. In the literature, it was explained that a polymer with lower inherent viscosity could get hydrated and swell better, and thus could release drug faster [221]. The in vitro depots of formulations 55 and 96 were significantly porous compared to the depot of 64 formulations. Figure 2-7 shows the pictures of in vitro depots of the formulations at two months. The order of porosity was $55>96>>64$. The in vitro depots of 55 and 96 formulations have shown signs of disintegration starting at 3 and 4 months, respectively. The depot of 55 showed quick disintegration and resulted in complete loss at about 4 months, while the depot of 96 was disintegrated by 5 months. Even through the depot of 55 constitute 100\% PLA (more hydrophobic than PLGA), but the higher porosity and faster disintegration of the depot of 55 over 96 could be attributed to the presence of lower inherent viscosity PLA polymer. 50\% of the polymer concentration used in 55 formulation was of lower inherent viscosity PLA $(0.25 \mathrm{iv})$, while the polymers used in 96 formulation were of higher inherent viscosity (PLGA-0.63/PLA-0.47 iv). On the other hand, the depot of the 64 formulation remained intact until the end of the study $(\sim 6.5$ months). The high amount of PLA with a higher inherent viscosity (0.63) used in 64 formulation might have shown slow degradation and extended the release for a longer time.

To understand the release mechanisms of LNG from the depots made of 55, 64 and 96 formulations, all the release data in Figure 2-6 were fitted using empirical power law (Korsmeyer-Peppas Model, Equation 2-5) [40] as shown below:

$$
\frac{M_{t}}{M_{\infty}}=k t^{n}
$$

Where $M_{t}$ and $M_{\infty}$ are the amounts of drug released at time $t$ and infinite (in this study the initial drug amount was used as $M_{\infty}$ ), respectively, $M_{t} / M_{\infty}$ is the fraction of drug released at time $t, k$ is the rate constant related to diffusion coefficient, and $n$ is the release exponent related to release mechanism. The correlations between $\mathrm{n}$ value and release mechanisms are: $n<0.5$, non-Fickian diffusion due to increasing hydrophobicity with time; $n=0.5$, Fickian diffusion; $0.5<n<0.1$, anomalous (combination of Fickian and 

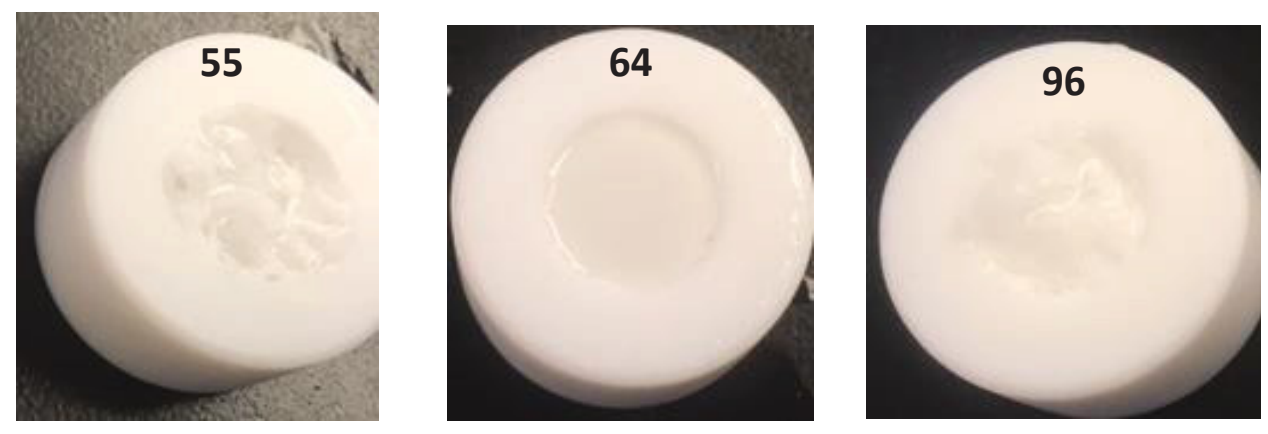

Figure 2-7. In vitro depot pictures of ISD formulations 55, 64 and 96 in Teflon molds after two months release study.

Notes: The porosity of the in vitro depots formed by the 55, 64, and 96 formulations was in the order of $55>96>>64$, and this order was correlated to the molecular weights of the polymers used in the formulations. 
Case II diffusion) diffusion; $n=1$, Case II diffusion due to polymer chain relaxation; and $n>1$, Super Case II diffusion due to polymer chain relaxation [40,41]. The Equation 2-5 has frequently been used to analyze the drug release from several modified polymeric drug delivery systems including both swellable and non-swellable systems $[41,42]$. The fitting parameters determined by the linear regression of log (percent cumulative release) against $\log t$ in for the LNG release from the depots made of 55, 64 and 96 formulations are given in Table 2-6. The release exponent $n$ values ranged from 0.46 to 0.69 suggesting different release mechanisms for the formulations 55, 64 and 96 . The evaluation of these three formulations was further continued to preclinical studies in rats to study the long-term in vivo LNG release.

\section{Plasma}

\section{LC-MS/MS Method Development and Validation for Analysis of LNG in Rat}

To conduct the preclinical pharmacokinetic studies of the LNG-loaded ISD formulations in rats, a sensitive and reliable bioanalytical method is needed to detect the in vivo drug concentrations. LC/MS/MS technique is a rapid, selective and sensitive analysis method that has been widely used for quantifying the concentration of a broad range of drugs in the plasma. There are several reports in the literature about using LC/MS/MS for detecting LNG from human plasma [222-225], environmental water, and sewage effluent [226-229]. However, there is no report about using LC/MS/MS for detecting LNG from small animal plasma. The method for detecting LNG from human plasma used the relatively large amount of plasma about $0.5-1 \mathrm{~mL}$. This volume of plasma is difficult to be obtained from small animals such as mice and rats. Hence, a highly sensitive LC/MS/MS analytical method with a lower limit of quantification (LLOQ) in the nanomolar or $\mathrm{ng} / \mathrm{mL}$ range needs to be developed. Therefore, a sensitive $\mathrm{LC} / \mathrm{MS} / \mathrm{MS}$ method was developed for bioanalysis of LNG over a concentration range of $0.5-50 \mathrm{ng} \cdot \mathrm{mL}^{-1}$ using limited volume $(100 \mu \mathrm{L})$ of rat plasma. The liquid-liquid extraction method was employed to extract LNG from rat plasma as it is a fast and convenient way for the extraction especially when a large number of samples are needed to be analyzed in short period of time. The liquid-liquid used in this study was the mixture of hexane and ethyl acetate which is relatively inexpensive and can be easily applied in both academic and industry settings. For reasonable accuracy and precision, the deuterated analog of

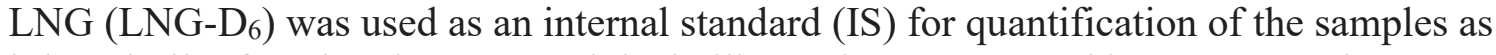
it has similar functional groups and the boiling point as LNG and is not a natural component of rat plasma. LNG was rapidly eluted and detected in the chromatogram in less than one minute. The developed method was validated for matrix effect, specificity, recovery efficiency, accuracy, precision, linearity and stability of the analyte LNG and published [230]. The validated method was further used to study the pharmacokinetics of LNG after SubQ injection in rats.

\section{LC/MS/MS method development}

Various sample pre-treatment or extraction methods were investigated to remove interfering compounds from rat plasma. Organic solvents such as methanol, hexane, ethyl acetate, chloroform, ether with different hydrophobicity and ratios were used to develop 
Table 2-6. Fitting parameters determined by the linear regression of $\log \left(M_{t} / M_{\infty}\right)$ against $\log t$ in Power law for the LNG release from the depots made of 55, 64 and 96 formulations.

\begin{tabular}{ccc}
\hline Formulation & Korsmeyer-Peppas $\left(\boldsymbol{M}_{\boldsymbol{t}} / \boldsymbol{M}_{\infty}=\boldsymbol{k} . \boldsymbol{t}^{\boldsymbol{n}}\right)$ \\
\cline { 2 - 3 }$\#$ & $\boldsymbol{R}^{\mathbf{2}}$ & $\boldsymbol{n}$ \\
\hline 55 & 0.99 & 0.69 \\
64 & 0.97 & 0.63 \\
96 & 0.99 & 0.46 \\
\hline
\end{tabular}


the liquid-liquid extraction procedure along with the evaluations for matrix effect, recovery efficiency, and reproducibility. Hexane/ethyl acetate $(80 / 20, \mathrm{v} / \mathrm{v})$ mixture was found to be efficient in terms of low matrix effect, high recovery percentile, and good peak reproducibility. Hexane/ether $(70 / 30, \mathrm{v} / \mathrm{v})$ mixture was also found to have good peak resolution and extraction efficiency. However, it had an issue with peak reproducibility due to the volatile nature of ether under the experimental conditions.

For better peak resolution and shorter run time, solvents such as methanol, acetonitrile and water were tested as mobile solvents in different combinations and concentrations. Better resolution of peaks was achieved with acetonitrile/water (70/30, $\mathrm{v} / \mathrm{v}$ ) mixture at a flow rate of $0.3 \mathrm{~mL} \cdot \mathrm{min}^{-1}$. Moreover, the addition of $0.01 \%$ formic acid in the mobile phase helped to obtain the smallest peak width and better peak asymmetry. To optimize ESI conditions for LNG and LNG- D6, scans were conducted in positive ion detection mode. The full scan mass spectrum of LNG was shown in Figure 2-8, revealing a peak at $\mathrm{m} / z 313.10$ correlated to protonated $\mathrm{LNG}$ ion $[\mathrm{M}+\mathrm{H}]+$, and other peaks correlated to fragmented ions which were obtained after the collision of the protonated ion. The fragmented ions 109.10 and 245.10 were used for specificity and sensitivity testing. The fragmentation $\mathrm{m} / \mathrm{z} 313.10 \rightarrow 245.10$ was used for identification of LNG and further quantification.

\section{Specificity}

The specificity was fully evaluated by screening the chromatograms of the blank plasma samples collected, plasma samples spiked with LNG and IS LNG- D6, and plasma samples collected from rats subcutaneously injected with LNG. Figure 2-9 illustrated representative LC/MS/MS chromatograms and demonstrated the absence of any chromatographic or mass spectrometric interference in the collected blank rat plasma samples. The retention time for the analyte LNG and IS LNG- D 6 were between 1.9 to $2.0 \mathrm{~min}$. No interference peaks were observed in any of the samples at the LNG and LNG- $\mathrm{D}_{6}$ retention times due to endogenous matrix components, indicating the efficient specificity of the conditions.

\section{Limits of detection and quantification and linearity}

In this work, the LOD and LLOQ for $\mathrm{LNG}$ extracted from $100 \mu \mathrm{L}$ of rat plasma were quantified to be $\sim 0.2$ and $0.5 \mathrm{ng} \cdot \mathrm{mL}^{-1}$ respectively. In the literature, there is no $\mathrm{LC} / \mathrm{MS} / \mathrm{MS}$ method reported for detecting LNG from small animal plasma although there are some papers on LC/MS/MS methods for detecting LNG from human plasma. Among the papers for detecting LNG from human plasma, LLOQ values of 0.265 [224], 0.25 [225], 0.1[222] and $0.05 \mathrm{ng} \cdot \mathrm{mL}^{-1}$ [223] were reported. Even though these LLOQ values for human plasma were lower than the LLOQ obtained in this work for rat plasma, they required five to ten times amount of plasma $(500-1000 \mu \mathrm{L}$ vs. $100 \mu \mathrm{L})$ which would be difficult to be obtained from small animals. Furthermore, the lowest LLOQ value 0.05 $\mathrm{ng} \cdot \mathrm{mL}^{-1}$ for human plasma was obtained by using solid phase extraction procedure [223] which was much more labor intensive than the simple liquid-liquid extraction procedure used in this work. Therefore, the existing LC/MS/MS methods for detecting LNG from 




Figure 2-8. The product ion mass spectrum of the LNG at $\mathbf{m} / \mathbf{z} 313.10$. 

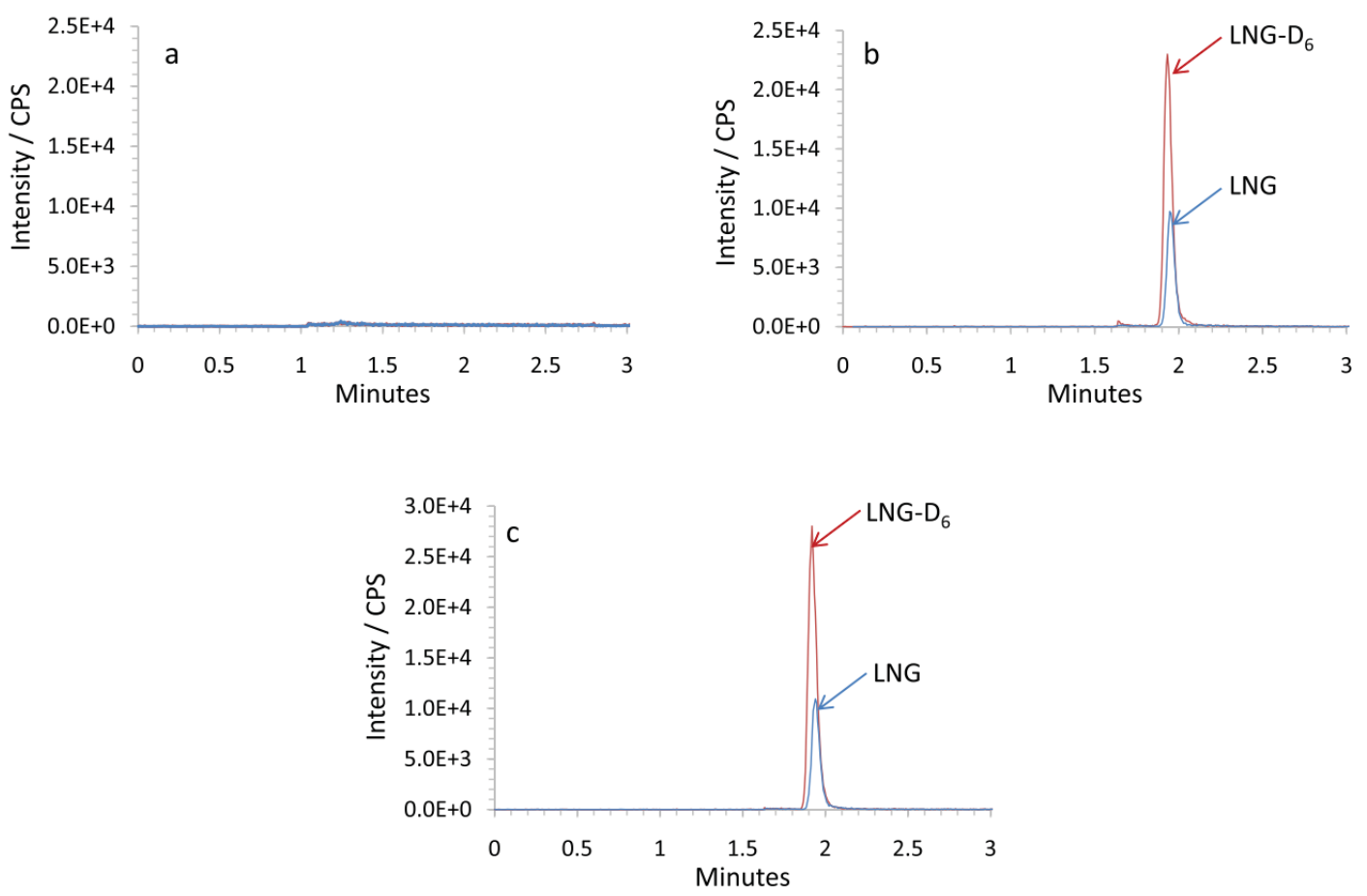

Figure 2-9. Representative chromatograms of LNG and internal standard (LNGD6).

Notes: (a) Blank plasma sample collected from a rat, (b) purchased plasma sample spiked with LNG and IS- LNG-D 6 , and (c) plasma sample collected from a rat after subcutaneously injected with LNG (spiked with IS). 
human plasma were not suitable for detecting LNG from small animal plasma. The $\mathrm{LC} / \mathrm{MS} / \mathrm{MS}$ method developed in this work is a new and highly sensitive method for detecting LNG from small animal plasma. The calibration lines showed good linearity (with $r^{2}=0.9996 \pm 0.003$ ) over the concentration range $0.5-50 \mathrm{ng} \cdot \mathrm{mL}^{-1}$ within six regression curves with high reliability and reproducibility. A typical regression equation $(n=6)$ was $y=(0.0515 \pm 0.0025) x+(0.0807 \pm 0.0079)$ and a characteristic calibration curve for LNG was shown in Figure 2-10. From the graph, the concentrations of the standard plasma samples were back-calculated for estimating the level of accuracy. Based on the calculations, it was found that the accuracy percentage for the back-calculated concertation of each calibration standard was within the acceptable $\pm 20 \%$ and $\pm 15 \%$ limits for the LLOQ and all the other plasma standard samples, respectively. Table 2-7 lists the characteristic calibration data for LNG.

\section{Accuracy and precision}

Seven standard samples $0.5,1,2.5,5,10,25$ and $50 \mathrm{ng} \cdot \mathrm{mL}^{-1}$ (Table 2-8 and Table 2-9) along with three QC samples LQC $\left(0.5 \mathrm{ng} \cdot \mathrm{mL}^{-1}\right)$, MQC $\left(5 \mathrm{ng} \cdot \mathrm{mL}^{-1}\right)$ and HQC $\left(50 \mathrm{ng} \cdot \mathrm{mL}^{-1}\right)$ (Table 2-9) were tested for intra-day and inter-day accuracy and precision. All the standard samples showed $90.88-110.33 \%, 88.67-107.09 \%$ and $86.09-104.16 \%$ intra-day accuracy and $0.38-5.59 \%, 0.66-3.09 \%$ and $0.36-3.62 \%$ intra-day CV on Day 1 , 2 and 3, respectively (Table 2-8). They also showed 92.97-107.03\% inter-day accuracy and $0.91-8.82 \%$ inter-day CV for 3 consecutive days (Table 2-8). The QC samples LQC, MQC and HQC had $112.60 \%, 96.31 \%$ and $107.00 \%$ intra-day accuracy and $2.52 \%$, $0.96 \%$ and $0.71 \%$ intra-day precision, respectively; and $105.27 \%, 86.75 \%$ and $100.32 \%$ inter-day accuracy and $2.20 \%, 1.64 \%$ and $0.67 \%$ inter-day precision, respectively (Table 2-10). The QC sample intra-day and inter-day accuracy and precision (CV) were within $\pm 20 \%$ for LLOQ and $\pm 15 \%$ for all the other concentrations, indicating satisfactory quality assurance for the accuracy and precision.

\section{Matrix effect}

The matrix effect was calculated for the three LNG QC samples LQC $\left(0.5 \mathrm{ng} \cdot \mathrm{mL}^{-}\right.$ $\left.{ }^{1}\right)$, MQC $\left(5 \mathrm{ng} \cdot \mathrm{mL}^{-1}\right)$ and HQC $\left(50 \mathrm{ng} \cdot \mathrm{mL}^{-1}\right)$ and internal standard LNG- D $6\left(25 \mathrm{ng} \cdot \mathrm{mL}^{-1}\right.$, given in Table 2-11). The matrix factors were $0.95,0.96$, and 0.96 for the LQC, MQC, and HQC samples, respectively, indicating that there was $4 \sim 5 \%$ chance of ion suppression due to the matrix. At the same time, matrix factor for the internal standard was 0.97 , indicating a $3 \%$ ion suppression effect.

\section{Recovery efficiency}

Based on the pre-extracted and post-extracted plasma standards, the recovery efficiency was found to be $90.80-92.65 \%$ for the three LNG QC samples LQC $(0.5$ $\left.\mathrm{ng} \cdot \mathrm{mL}^{-1}\right)$, MQC $\left(5 \mathrm{ng} \cdot \mathrm{mL}^{-1}\right)$ and HQC $\left(50 \mathrm{ng} \cdot \mathrm{mL}^{-1}\right)$ and $96.76 \%$ for the internal standard LNG- $\mathrm{D}_{6}$ (Table 2-11). Being non-polar in nature, hexane/ethyl acetate mixture (80/20, $\mathrm{v} / \mathrm{v}$ ) was efficient to extract the hydrophobic LNG and LNG- D6 with little interference of the matrix components. 


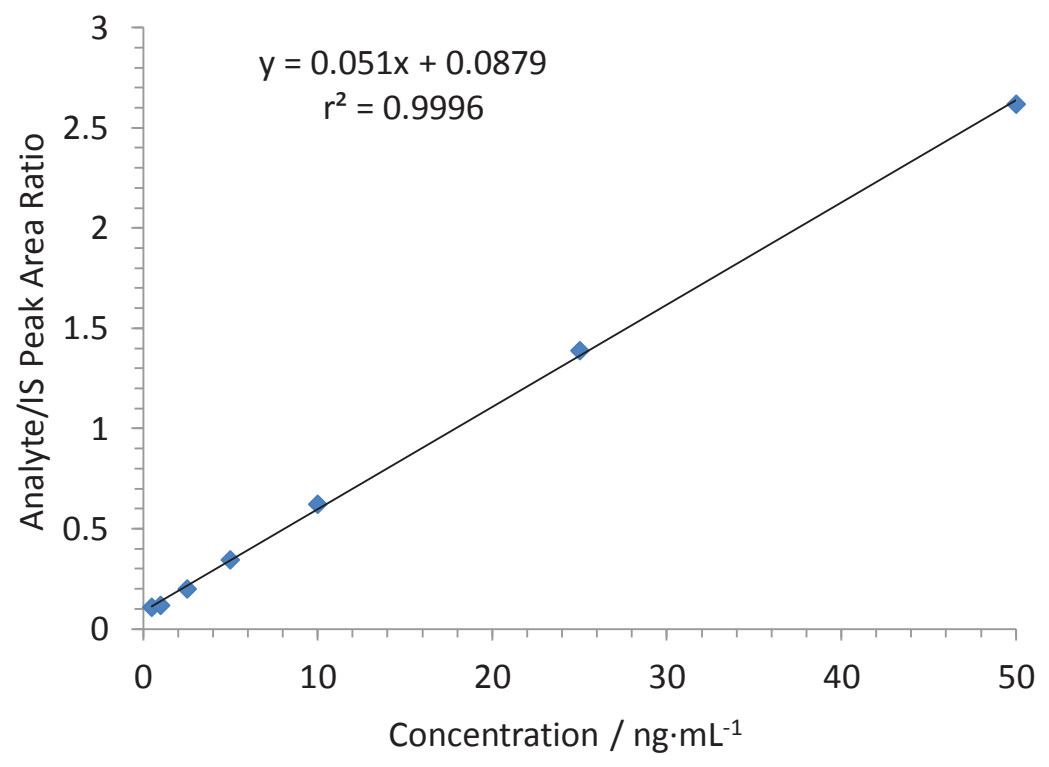

Figure 2-10. Representative calibration curve for LNG in rat plasma. 
Table 2-7. Linearity and summary of calibration curves for LNG in rat plasma $(n=6)$ with back-calculated concentrations.

\begin{tabular}{cccccc}
\hline $\begin{array}{c}\text { Concentration } \\
\mathbf{n g} \cdot \mathbf{m L}^{-\mathbf{1}}\end{array}$ & $\begin{array}{c}\text { Mean calculated } \\
\text { concentration } \\
\mathbf{n g} \cdot \mathbf{m L}^{-\mathbf{1}}\end{array}$ & SD & $\begin{array}{c}\text { Mean } \\
\mathbf{a c c u r a c y} \\
\mathbf{\%}\end{array}$ & SD & $\begin{array}{c}\mathbf{C V} \\
\mathbf{\%}\end{array}$ \\
\hline 0.5 & 0.466 & 0.022 & 93.2 & 4.43 & 4.75 \\
1 & 1.02 & 0.049 & 102.2 & 4.91 & 4.80 \\
2.5 & 2.46 & 0.093 & 98.2 & 3.71 & 3.78 \\
5 & 5.20 & 0.250 & 104.0 & 5.01 & 4.81 \\
10 & 10.3 & 0.358 & 103.4 & 3.58 & 3.47 \\
25 & 24.9 & 0.613 & 99.6 & 2.45 & 2.46 \\
50 & 49.5 & 0.952 & 99.0 & 1.90 & 1.92 \\
\hline
\end{tabular}


Table 2-8. Intra-day assay accuracy and precision determination of LNG standards in rat plasma for 3 consecutive days $(n=6)$.

\begin{tabular}{|c|c|c|c|c|c|}
\hline Day & $\begin{array}{c}\text { Concentration } \\
\mathbf{n g} \cdot \mathbf{m L}^{-1}\end{array}$ & $\begin{array}{c}\text { Mean calculated } \\
\text { concentration } \\
\mathrm{ng} \cdot \mathrm{mL}^{-1}\end{array}$ & SD & $\begin{array}{c}\text { Mean } \\
\text { accuracy } \\
\%\end{array}$ & $\begin{array}{l}\mathrm{CV} \\
\%\end{array}$ \\
\hline 1 & 0.5 & 0.465 & 0.026 & 93.00 & 5.59 \\
\hline 1 & 1 & 1.103 & 0.011 & 110.33 & 1.02 \\
\hline 1 & 2.5 & 2.272 & 0.036 & 90.88 & 1.60 \\
\hline 1 & 5 & 4.865 & 0.067 & 97.31 & 1.39 \\
\hline 1 & 10 & 10.983 & 0.078 & 109.83 & 0.71 \\
\hline 1 & 25 & 25.033 & 0.379 & 100.13 & 1.51 \\
\hline 1 & 50 & 50.648 & 0.191 & 101.30 & 0.38 \\
\hline 2 & 0.5 & 0.472 & 0.015 & 94.47 & 3.09 \\
\hline 2 & 1 & 0.968 & 0.020 & 96.80 & 2.09 \\
\hline 2 & 2.5 & 2.217 & 0.015 & 88.67 & 0.66 \\
\hline 2 & 5 & 4.776 & 0.058 & 95.51 & 1.22 \\
\hline 2 & 10 & 10.709 & 0.076 & 107.09 & 0.71 \\
\hline 2 & 25 & 24.679 & 0.213 & 98.72 & 0.86 \\
\hline 2 & 50 & 49.782 & 0.441 & 99.56 & 0.89 \\
\hline 3 & 0.5 & 0.499 & 0.018 & 99.80 & 3.62 \\
\hline 3 & 1 & 0.937 & 0.025 & 93.70 & 2.67 \\
\hline 3 & 2.5 & 2.495 & 0.047 & 99.79 & 1.89 \\
\hline 3 & 5 & 4.304 & 0.016 & 86.09 & 0.36 \\
\hline 3 & 10 & 10.416 & 0.103 & 104.16 & 0.99 \\
\hline 3 & 25 & 24.517 & 0.259 & 98.07 & 1.06 \\
\hline 3 & 50 & 49.974 & 0.232 & 99.95 & 0.46 \\
\hline
\end{tabular}


Table 2-9. Inter-day assay accuracy and precision determination of LNG standards in rat plasma $(n=18)$.

\begin{tabular}{ccccc}
\hline $\begin{array}{c}\text { Concentration } \\
\mathbf{n g} \cdot \mathbf{m L}^{-\mathbf{1}}\end{array}$ & $\begin{array}{c}\text { Mean calculated } \\
\text { concentration } \\
\mathbf{n g} \cdot \mathbf{m L}^{-\mathbf{1}}\end{array}$ & SD & $\begin{array}{c}\text { Mean } \\
\mathbf{a c c u r a c y} \\
\mathbf{\%}\end{array}$ & $\begin{array}{c}\mathbf{C V} \\
\mathbf{\%}\end{array}$ \\
\hline 0.5 & 0.479 & 0.018 & 95.76 & 3.74 \\
1 & 1.003 & 0.088 & 100.28 & 8.82 \\
2.5 & 2.328 & 0.147 & 93.11 & 6.32 \\
5 & 4.648 & 0.301 & 92.97 & 6.48 \\
10 & 10.703 & 0.284 & 107.03 & 2.65 \\
25 & 24.743 & 0.264 & 98.97 & 1.07 \\
50 & 50.135 & 0.455 & 100.27 & 0.91 \\
\hline
\end{tabular}


Table 2-10. Accuracy and precision for LNG QC samples in rat plasma.

\begin{tabular}{lccccc}
\hline Day & $\begin{array}{c}\text { Concentration } \\
\mathbf{n g} \cdot \mathbf{m L}^{-1}\end{array}$ & $\begin{array}{c}\text { Mean calculated } \\
\text { concentration } \\
\mathbf{n g} \cdot \mathbf{m L}^{-1}\end{array}$ & SD & $\begin{array}{c}\text { Mean } \\
\text { accuracy } \\
\text { \% }\end{array}$ & $\begin{array}{c}\mathbf{C V} \\
\%\end{array}$ \\
\hline Intra-day $(\mathrm{n}=6)$ & 0.5 & 0.563 & 0.014 & 112.60 & 2.52 \\
Intra-day $(\mathrm{n}=6)$ & 5 & 4.816 & 0.046 & 96.31 & 0.96 \\
Intra-day (n=6) & 50 & 53.498 & 0.382 & 107.00 & 0.71 \\
Inter-day (n=18) & 0.5 & 0.526 & 0.012 & 105.27 & 2.20 \\
Inter-day (n=18) & 5 & 4.338 & 0.071 & 86.75 & 1.64 \\
Inter-day (n=18) & 50 & 50.160 & 0.338 & 100.32 & 0.67 \\
\hline
\end{tabular}


Table 2-11. Matrix effect and recovery efficiency results of LNG QC samples and LNG-D6.

\begin{tabular}{lcccccc}
\hline Analyte & $\begin{array}{c}\text { Concentration } \\
\mathbf{n g} \cdot \mathbf{m L}^{-1}\end{array}$ & $\begin{array}{c}\text { Spiked in } \\
\text { methanol }\end{array}$ & $\begin{array}{c}\text { Spiked } \\
\text { before } \\
\text { extraction }\end{array}$ & $\begin{array}{c}\text { Spiked } \\
\text { after } \\
\text { extraction }\end{array}$ & $\begin{array}{c}\text { Matrix } \\
\text { factor }\end{array}$ & $\begin{array}{c}\text { Recovery } \\
\text { efficiency } \\
\text { \%o }\end{array}$ \\
\hline LNG & $0.5(\mathrm{LQC})$ & $1.47 \mathrm{E}+04$ & $1.27 \mathrm{E}+04$ & $1.40 \mathrm{E}+04$ & 0.95 & 90.80 \\
LNG & $5(\mathrm{MQC})$ & $8.91 \mathrm{E}+04$ & $7.98 \mathrm{E}+04$ & $8.62 \mathrm{E}+04$ & 0.96 & 92.65 \\
LNG & $50(\mathrm{HQC})$ & $6.13 \mathrm{E}+05$ & $5.50 \mathrm{E}+05$ & $5.94 \mathrm{E}+05$ & 0.96 & 92.64 \\
LNG-D & 25 & $6.42 \mathrm{E}+04$ & $6.05 \mathrm{E}+04$ & $6.26 \mathrm{E}+04$ & 0.97 & 96.76 \\
\hline
\end{tabular}




\section{Stability}

Lack of stability results in non-reproducible outputs during the analysis. Hence, the stability of the analyte during the experimental conditions, from the stage of storage to chromatography analysis is necessary. In this stability study, five experimental conditions were used for three LNG QC samples LQC $\left(0.5 \mathrm{ng} \cdot \mathrm{mL}^{-1}\right)$, MQC $\left(5 \mathrm{ng} \cdot \mathrm{mL}^{-1}\right)$ and HQC (50 ng $\left.\mathrm{mL}^{-1}\right)$ and the results were shown in Table 2-12. Long term stability mean accuracy of LNG stored at $-80{ }^{\circ} \mathrm{C}$ (storage condition) for 15 days and under 12 cycles of freeze-thaw process from $-80{ }^{\circ} \mathrm{C}$ to room temperature was $111.07 \%, 95.65 \%$, and $98.60 \%$; and $108.53 \%, 97.45 \%$ and $100.17 \%$ for LQC, MQC, and HQC samples, respectively. In-process stability mean accuracy of LNG stored in the reconstitution solvent methanol/water $(50 / 50, \mathrm{v} / \mathrm{v})$ mixture at room temperature for $4 \mathrm{~h}$ was $113 \%$, $96.72 \%$ and $104.25 \%$ for LQC, MQC, and HQC samples, respectively. Postoperative stability mean accuracy of LNG after keeping the final processed samples at room temperature for $8 \mathrm{~h}$ at $10{ }^{\circ} \mathrm{C}$ (autosampler injector temperature) for $24 \mathrm{~h}$ was $102.93 \%$, 91.57\%, and $98.15 \%$; and $114.4 \%, 102.72 \%$ and $105.76 \%$ for LQC, MQC, and HQC samples, respectively. All the QC samples showed $91.57-114.4 \%$ stability mean accuracy values in the acceptable quality limit under the five experimental conditions.

\section{Application to pharmacokinetic study in rats}

The developed LC/MS/MS method was successfully applied to quantify LNG concentration in the rat plasma after LNG in $400 \mu \mathrm{L}$ NMP solution $(4 \mathrm{mg} / \mathrm{rat})$ was subcutaneously injected into female SD rats $(\mathrm{n}=6)$. The calculated $\mathrm{LNG}$ concentrations in rat plasma were graphed as a function of time for two days of pharmacokinetics study, as shown in Figure 2-11. The pharmacokinetic parameters of LNG were calculated by noncompartmental analysis and listed in Table 2-13, which showed that $C_{\max }, A U C_{(0-48 h r s)}$, $t 1 / 2, V d / F$ and $C L / F$ were $229.17 \pm 49.23 \mathrm{ng} \cdot \mathrm{mL}^{-1}, 888.85 \pm 229.17 \mathrm{ng} \cdot \mathrm{h} \cdot \mathrm{mL}^{-1}, 22.07 \pm$ $4.97 \mathrm{~h}, 424.94 \pm 130.29 \mathrm{~L} \cdot \mathrm{kg}^{-1}$, and $16.36 \pm 3.12 \mathrm{~L} \cdot \mathrm{h}^{-1} \cdot \mathrm{kg}^{-1}$, respectively. The $t_{1 / 2}$ obtained here was well in agreement with the literature reported $t_{1 / 2}\left(23 \mathrm{~h}^{-1}\right)$ obtained by radioimmunoassay [231].

\section{In Vivo Evaluation of the Selected ISD Formulations (55, 64 and 96)}

Following the in vitro assessment of the ISD formulations, the 55, 64 and 96 formulations have been chosen for SubQ administration into the right flank of rats at 10 $\mathrm{mg}$ per rat (roughly $40 \mathrm{mg} / \mathrm{kg}$ ) for in vivo evaluation. Figure 2-12 illustrates the longterm plasma LNG profiles of the formulations 55, 64 and 96 starting from day 1 to day 210. 55 and 96 formulations released LNG constantly resulting in rat plasma concentration of $1-3 \mathrm{ng} \cdot \mathrm{mL}^{-1}(\mathrm{n}=8)$ up to 3 and 5 months, respectively and even extended to 7 months in two rats. On the other hand, 64 formulation released LNG continuously resulting in rat plasma concentration $0.5-3 \mathrm{ng} \cdot \mathrm{mL}^{-1}(\mathrm{n}=8)$ for 7 months or longer. Similar to the in vitro release results, 64 formulation showed sustained release in vivo, which could be due to the denser depot and slower degradation of the higher molecular weight $(103 \mathrm{kDa})$ PLA polymer in 64 formulation. The in vivo study showed that the 
Table 2-12. Stability of LNG QC samples at the various experimental condition.

\begin{tabular}{|c|c|c|c|c|c|c|}
\hline \multirow[b]{2}{*}{ Condition } & \multicolumn{2}{|c|}{ LQC } & \multicolumn{2}{|c|}{ MQC } & \multicolumn{2}{|c|}{ HQC } \\
\hline & $\begin{array}{c}\text { Calculated } \\
\text { concentration } \\
\text { mean } \pm \text { SD } \\
\mathbf{n g} \cdot \mathbf{m L}^{-1}\end{array}$ & $\begin{array}{c}\text { Mean } \\
\text { accuracy } \\
\%\end{array}$ & $\begin{array}{c}\text { Calculated } \\
\text { concentration } \\
\text { mean } \pm \text { SD } \\
\mathbf{n g} \cdot \mathbf{m L}^{-1}\end{array}$ & $\begin{array}{c}\text { Mean } \\
\text { accuracy } \\
\%\end{array}$ & $\begin{array}{c}\text { Calculated } \\
\text { concentration } \\
\text { mean } \pm \text { SD } \\
\mathbf{n g} \cdot \mathbf{m L} \mathbf{L}^{-1}\end{array}$ & $\begin{array}{c}\text { Mean } \\
\text { accuracy } \\
\%\end{array}$ \\
\hline Freezer $\left(-80^{\circ} \mathrm{C}\right)$ & $0.555 \pm 0.032$ & 111.07 & $4.783 \pm 0.139$ & 95.65 & $49.300 \pm 0.110$ & 98.60 \\
\hline Freeze \& Thaw ( 5 cycles $)$ & $0.543 \pm 0.021$ & 108.53 & $4.873 \pm 0.136$ & 97.45 & $50.087 \pm 1.280$ & 100.17 \\
\hline $\begin{array}{l}\text { In the rat plasma at room } \\
\text { temperature for } 4 \mathrm{~h}\end{array}$ & $0.565 \pm 0.026$ & 113 & $4.836 \pm 0.074$ & 96.72 & $52.127 \pm 1.300$ & 104.25 \\
\hline $\begin{array}{l}\text { In the reconstitution solvent } \\
\text { stored at room temperature for } 8 \mathrm{~h}\end{array}$ & $0.515 \pm 0.024$ & 102.93 & $4.578 \pm 0.235$ & 91.57 & $49.077 \pm 1.612$ & 98.15 \\
\hline $\begin{array}{l}\text { In the reconstitution solvent } \\
\text { stored in injector at } 10^{\circ} \mathrm{C} \text { for } 24 \mathrm{~h}\end{array}$ & $0.572 \pm 0.031$ & 114.4 & $5.136 \pm 0.100$ & 102.72 & $52.879 \pm 0.578$ & 105.76 \\
\hline
\end{tabular}




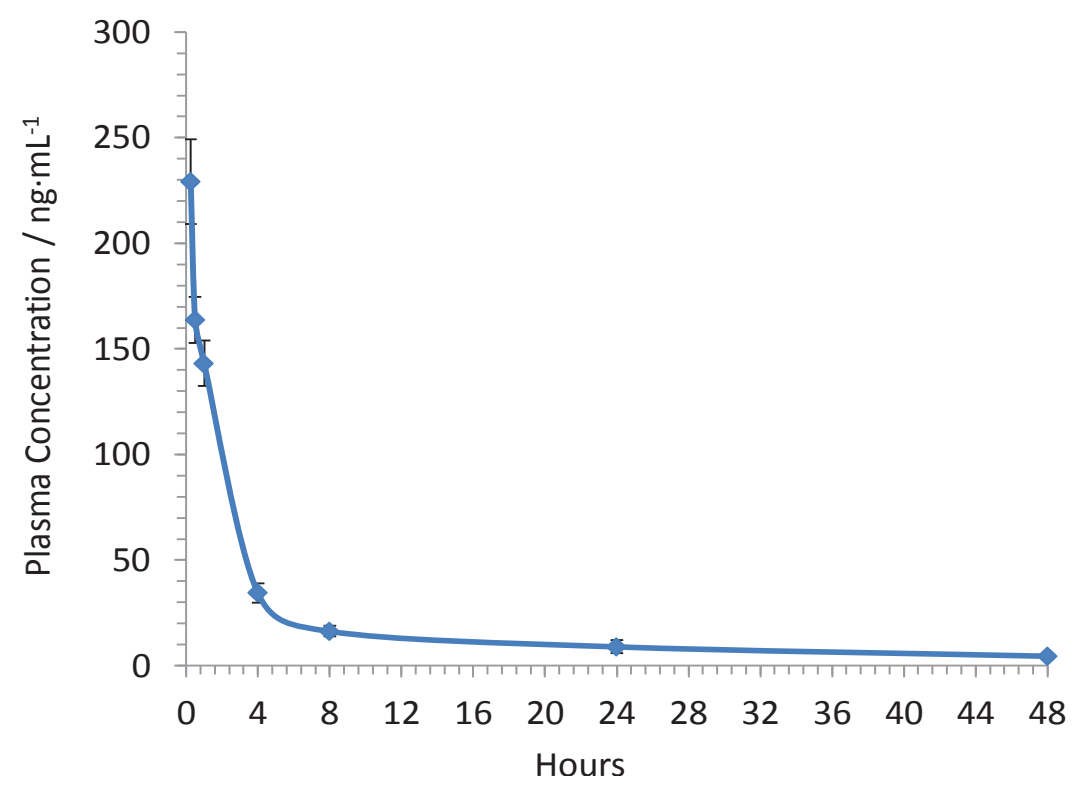

Figure 2-11. LNG concentration in rat plasma $(n=6)$ as a function of time after SubQ injection of LNG solution in NMP $\left(16 \mathrm{mg} \cdot \mathrm{kg}^{-1}\right)$. 
Table 2-13. Pharmacokinetic parameters of $L N G$ in rat plasma $(n=6)$ after SubQ injection of LNG in NMP solution $\left(16 \mathrm{mg} \cdot \mathrm{kg}^{-1}\right)$.

\begin{tabular}{|c|c|c|c|c|c|c|}
\hline Parameters & $\begin{array}{l}A U C_{(0-48 h r s)} \\
\mathbf{n g} \cdot h \cdot \mathbf{m L}^{-1}\end{array}$ & $\begin{array}{c}C_{\max } \\
\mathrm{ng} \cdot \mathrm{mL}^{-1}\end{array}$ & $\begin{array}{c}T_{\max } \\
\mathrm{h}\end{array}$ & $\begin{array}{c}t_{1 / 2} \\
h\end{array}$ & $\begin{array}{c}C L / F \\
\mathrm{~L} \cdot \mathbf{h}^{-1} \cdot \mathrm{kg}^{-1}\end{array}$ & $\begin{array}{c}V d / F \\
\mathbf{L} \cdot \mathbf{k g}^{-1}\end{array}$ \\
\hline Average & 888.85 & 229.17 & 0.25 & 22.07 & 16.36 & 424.94 \\
\hline SD & 200.54 & 49.23 & & 4.97 & 3.12 & 130.29 \\
\hline
\end{tabular}




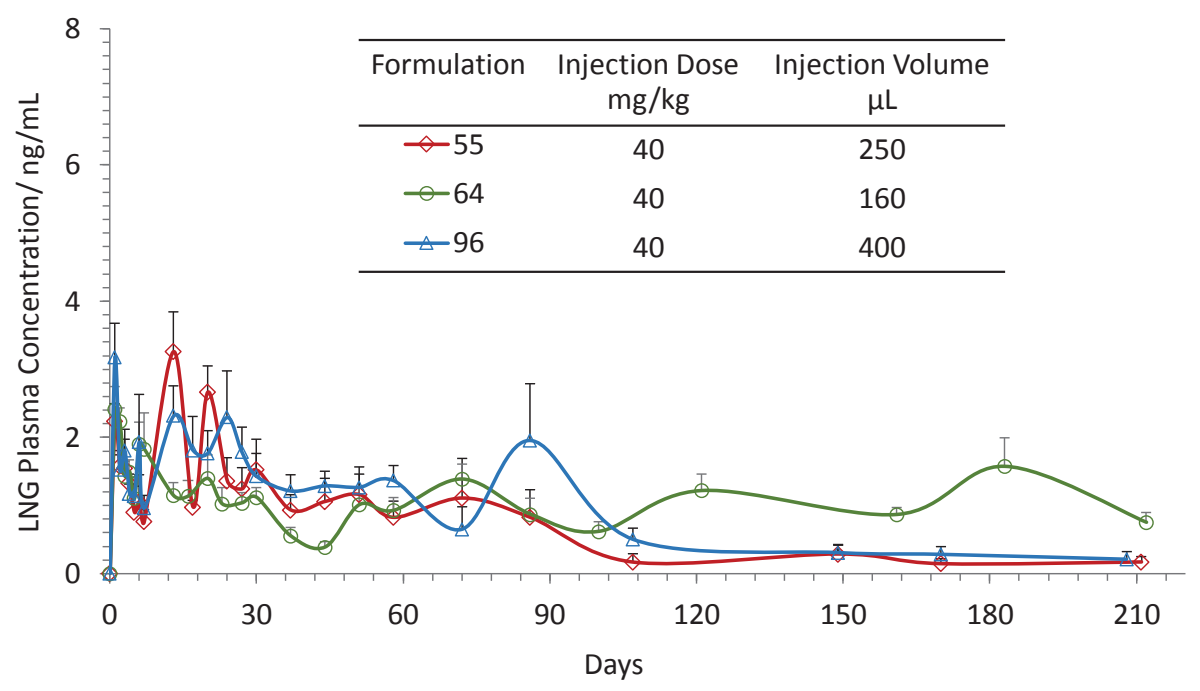

Figure 2-12. Plasma concentration of LNG (long-term levels) as a function of time after SubQ injections of ISD formulations.

Notes: The LNG containing ISD formulations 55 (open diamond), 64 (open circle), and 96 (open triangle) were injected subcutaneously into female SD rats at dose of $10 \mathrm{mg}$ per rat ( $40 \mathrm{mg} . \mathrm{kg}$ ), and the plasma samples were analyzed for LNG levels over a period of $\sim 7$ months. Each point represents the mean \pm SE $(n=8) .55$ and 96 formulations showed the robust release of LNG for 3 and 5 months, respectively while 64 formulation released LNG continuously for 7 months or longer. 
formulations maintained drug levels above the threshold $\left(\sim 0.2 \mathrm{ng} \cdot \mathrm{mL}^{-1}\right)$ to maintain the contraception in rats for prolonged time [232]. No significant swelling or abscessation was observed at the injection site; further, histology study of the injection site at the end of the study did not reveal any significant interactions. Monitoring the rat weights during the treatment period showed that the rats injected with LNG gained more weight than the control rats (Figure 2-13), and this behavior was consistent with the LNG-induced weight gain data in humans [233]. Both 96 (5 months) and 64 ( $>7$ months) formulations have successfully outperformed the current existing injectable contraceptives (3 months) in terms of therapeutic duration. Further, LNG is superior to medroxyprogesterone (API in Depo-Provera) regarding the bone loss $[1,2]$. Therefore, injectable contraceptives of LNG stands as a promising alternative to the existing injectable LARCs.

Apart from the pharmacokinetic evaluation, the efficacy of the formulations was also examined by studying the vaginal secretions of the rats injected with LNG. Unmated lab female rats show cyclic changes in the vaginal cytology through the span of the estrous cycle, and these progressions are biological markers of variances in the release of the ovarian hormones. In most non-pregnant lab rats, a complete cycle of such changes happens each 4 or 5 days [234]. Figure 2-14 shows the cyclic changes in the estrous cycle represented by the vaginal cytology images. Normal estrous cycle progression in rats is 4 day (M D P E M) or 5 day (D D P E D or M D D E M). Periodical examination of the vaginal secretions of the rats after injecting with the formulations revealed that the rats were acyclic indicating that formulations successfully suppressed the normal estrous cycles in the rats. Figure 2-15 shows representative images of vaginal cytology after injecting the formulation (96) for consecutive days showing acyclic progression. The group of rats injected with 96 formulation was selected, and the vaginal examination was continued after five months to demonstrate if the LNG-loaded ISD formulation effects are reversible in the rats after the end of the treatment. The results revealed that the rats resumed estrous cycles after clearance of the LNG indicating that the effects of the formulations are reversible. Further, when these female rats were co-caged with male rats for fertility study, the results demonstrated that 6 out of 8 rats become pregnant (\#96) after clearance of the LNG at about 8 months after initial injection suggesting the return of fertility after the treatment.

\section{Initial In Vivo Burst Study of 64 and 96 Formulations}

One of the primary limitations of ISD formulations is the initial burst, so it is essential to analyze the initial burst drug levels in plasma for ISD formulations. As 55 formulation showed poor long-term in vivo performance, only 64 and 96 formulations were selected to conduct the in vivo initial burst study. A short-term pharmacokinetic study was conducted where the drug levels were detected immediately during the early time intervals within $24 \mathrm{~h}$ after injecting the formulations (64 and 96). The initial burst levels of the formulations were detected and compared to the data obtained by injecting LNG solution alone (in NMP), (Figure 2-16). The $C_{\max }, T_{\max }$ and $A U C_{(0-24 h)}$ of the drug solution and formulations is given in Table 2-14. LNG solution in NMP without the polymers has resulted in a $C_{\max }$ of $231.7 \pm 17.8 \mathrm{ng} \cdot \mathrm{mL}^{-1}$ and $A U C_{(0-24 h)}$ of $725.4 \pm 45.5$ $\mathrm{ng} \cdot \mathrm{hr} \cdot \mathrm{mL}^{-1}(\mathrm{n}=6)$ at a dose of $4 \mathrm{mg}$ per rat (roughly $16 \mathrm{mg} / \mathrm{kg}$ ), while the presence of 


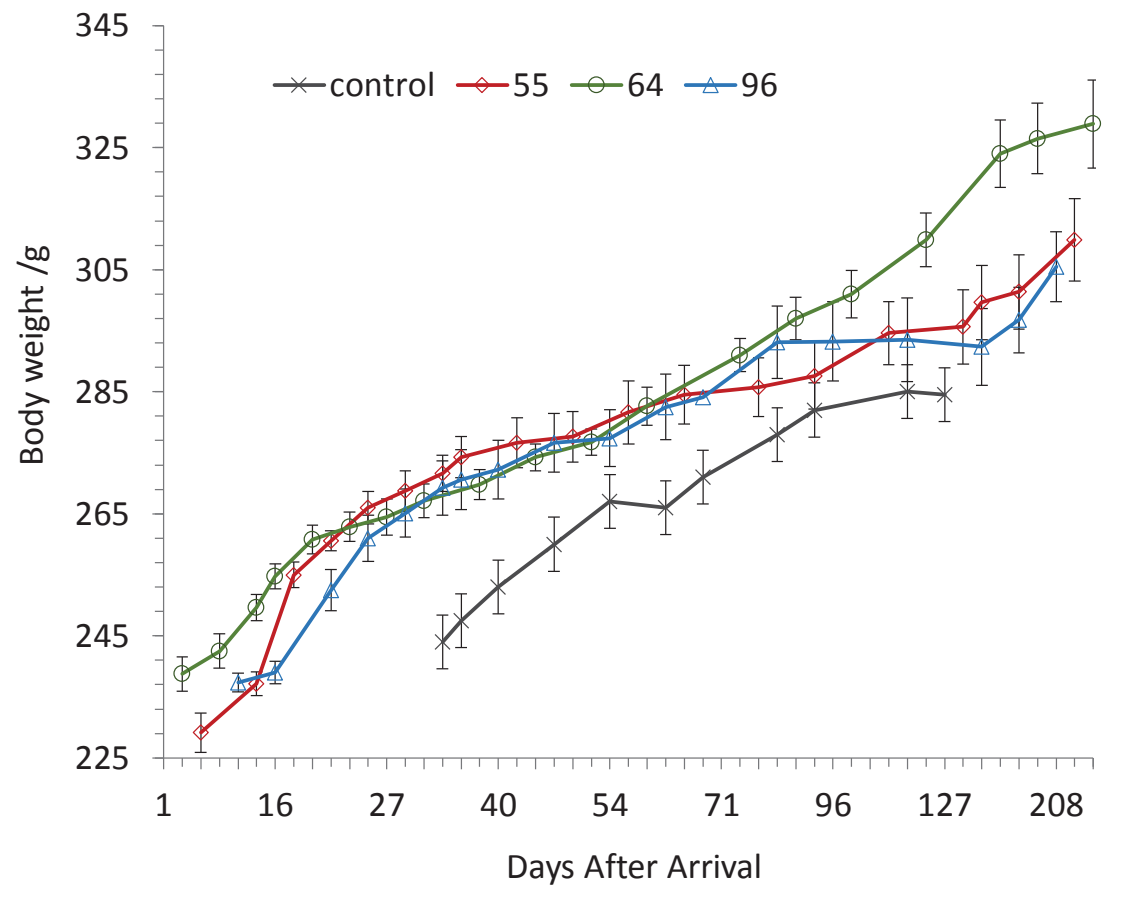

Figure 2-13. Changes in body weights of rats subcutaneously injected with ISD formulations.

Notes: The LNG containing ISD formulations ( $\mathrm{n}=8) 55$ (open diamond), 64 (open circle), and 96 (open triangle) were injected subcutaneously into female SD rats at a dose of 10 $\mathrm{mg}$ per rat $(\sim 40 \mathrm{mg} . \mathrm{kg})$ and the body weights monitored over a period $\sim 7$ months. The control rat weights are represented by cross mark $(n=2)$. Each point represents the mean \pm SE. The rats injected with LNG showed increased weight gain compared to the control. 


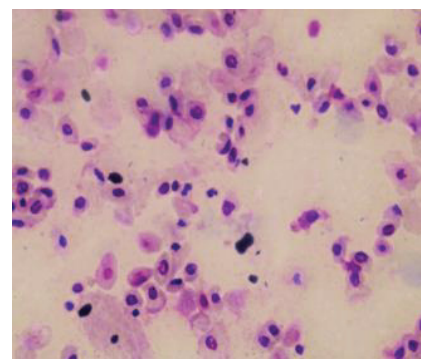

Proestrus

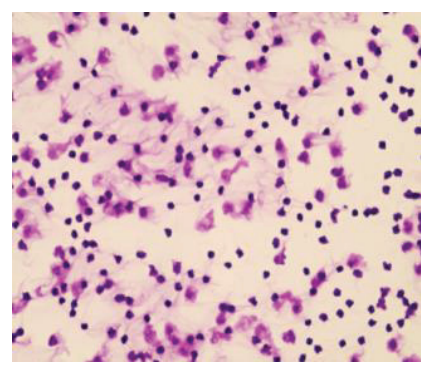

Metestrus



Estrus

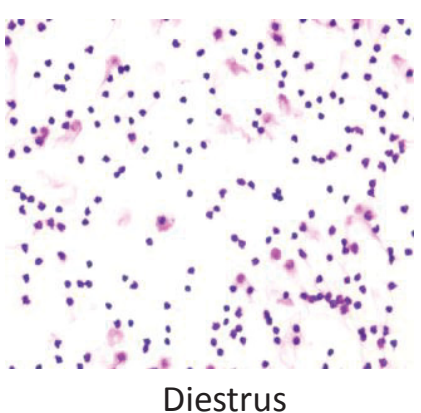

Figure 2-14. Four stages of rat estrous cycle and characteristic features.

Notes: Proestrus (P) - nucleated epithelial cells; Estrus (E) - anucleated cornified cells; Metestrus (M) -leukocytes, cornified, and nucleated epithelial cells; Diestrus (D) leukocytes 


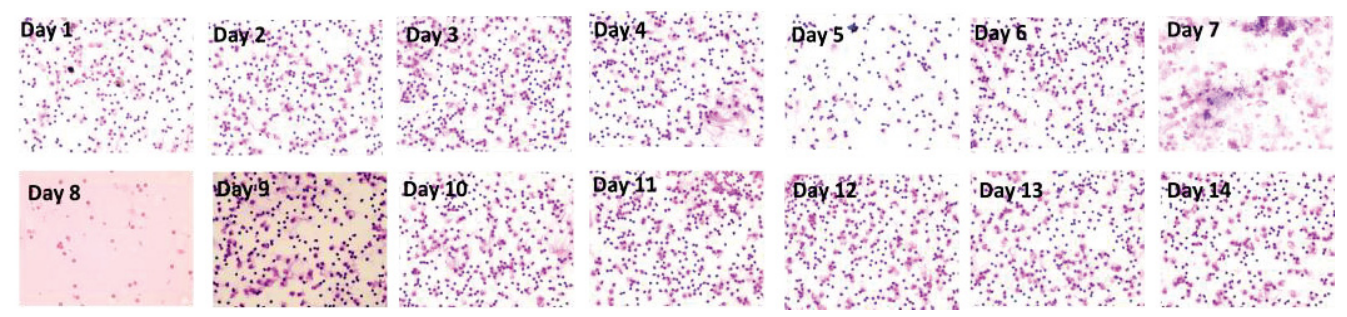

Figure 2-15. Representative image of the cycle progression in rats injected with formulation (F-96).

Notes: Cycle Progression: DDDDDDPEMDDDDDD, representing acyclic. 


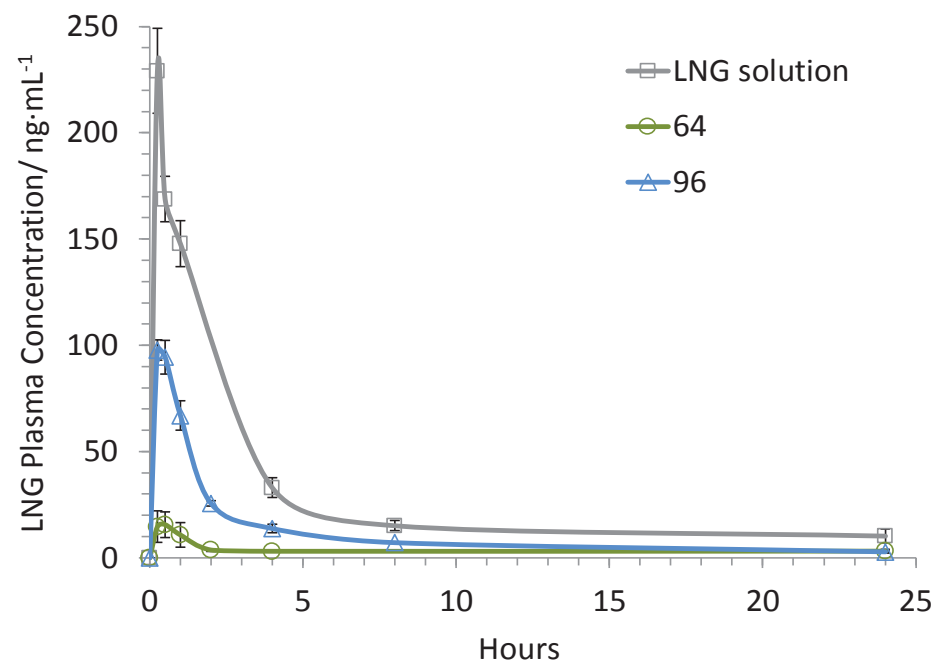

Figure 2-16. Plasma concentration of LNG (initial burst levels) in female SD rats as a function of time over $\mathbf{2 4} \mathrm{h}$ after SubQ injection of ISD formulations and control LNG.

Notes: LNG solution: $4 \mathrm{mg}$ LNG in $400 \mu \mathrm{L}$ NMP (square, $\mathrm{n}=6$ ); 64 formulation (circle, $\mathrm{n}=5$ ), and 96 (triangle, $\mathrm{n}=3$ ) formulations containing $10 \mathrm{mg} \mathrm{LNG}$. Each point represents the mean $\pm \mathrm{SE}$. Formulations showed a significant suppression of $C_{\max }$ and $A U C_{(0-24 h)}$ compared to the control LNG solution. 
Table 2-14. Pharmacokinetic parameters of LNG solution in NMP, 64 and 96 formulations in rat plasma after SubQ injection.

\begin{tabular}{|c|c|c|c|c|}
\hline $\begin{array}{l}\text { Formulation } \\
\#\end{array}$ & $\begin{array}{l}\text { Injection dose } \\
\mathrm{mg} \cdot \mathbf{K g}^{-1}\end{array}$ & $\begin{array}{c}C_{\max } \\
\mathbf{n g} \cdot \mathbf{m L}^{-1}\end{array}$ & $\begin{array}{c}T_{\max } \\
\mathbf{h}\end{array}$ & $\begin{array}{l}A U C_{(0-24 h)} \\
\mathrm{ng} \cdot \mathbf{h r} \cdot \mathbf{m L}^{-1}\end{array}$ \\
\hline LNG in NMP $(n=6)$ & 16 & $231.7 \pm 17.8$ & $0.29 \pm 0.04$ & $725.4 \pm 45.5$ \\
\hline $64(n=5)$ & 40 & $20.8 \pm 7.88$ & $0.35 \pm 0.06$ & $35.6 \pm 9.13$ \\
\hline $96(n=3)$ & 40 & $103.6 \pm 2.88$ & $0.33 \pm 0.08$ & $319.0 \pm 20.2$ \\
\hline
\end{tabular}


polymers in the formulation has significantly suppressed the amount of LNG release into the systemic circulation even though the dose $(10 \mathrm{mg} / \mathrm{rat})$ was 1.5 times higher compared to the LNG solution. 64 and 96 formulations resulted in a $C_{\max }$ of $20.8 \pm 7.9 \mathrm{ng} / \mathrm{mL}(\mathrm{n}=5)$, and $103.6 \pm 2.9 \mathrm{ng} \cdot \mathrm{mL}^{-1}(\mathrm{n}=3)$, respectively, meanwhile the $A U C_{(0-24 h)}$ were found to be $35.6 \pm 9.13$ and $319.0 \pm 20.2$, respectively. 64 formulation has shown significant lower burst ( 5 times lower $C_{\max }$ ) compared to the 96 formulation, and the reduced initial burst might be due to the altered phase inversion dynamics $[184,220]$ as a result of increased polymer molecular weight (102 $\mathrm{kDa}$ vs $63 \mathrm{kDa})$, drug loading (6\% vs 2.5\%), injection volume $(160 \mu \mathrm{L}$ vs $400 \mu \mathrm{L})$, and change in solvent (NMP/TEC vs NMP/BB), but the primary factor is assumed to be the polymer MW. Polymer MW is a critical parameter that can affect the drug release and it is reported in the literature that increased polymer molecular weight has shown a significant lower burst because of the formation of the denser structure of the depot $[184,235,236]$. On the other hand, the lower molecular weight PLA used in 96 formulation resulted in reduced viscosity $(0.67 \pm 0.05 \mathrm{~Pa} . \mathrm{s}$ vs. $1.71 \pm 0.01 \mathrm{~Pa} . \mathrm{s}$ for 64 ) causing more faster release of organic solvent and LNG, which might lead to a fast phase inversion type depot formation having greater porosity and fast release [237]. Other factors that may have involved for the initial burst in vivo for the formulations is the interstitial pressure/compressive forces exerted on the depot by the surrounding tissue environment which forces the solvent and drug to efflux out fast increasing the rate of depot formation in vivo [185, 238]. Although the polymer percentage $(\sim 24 \%)$ used in the prototype formulations is lesser than that employed in Eligard $^{\circledR}$ (33-44\%), still the formulations showed promising in vivo burst profile, suggesting that the use of polymer blend has a favorable effect. Additionally, the use of polymer blend and low polymer concentration (24\%) has enabled to incorporate high molecular weight polymer into the formulations to achieve low burst while maintaining the favorable injectability $(21 / 23 \mathrm{G})$.

Summary of the observations based on the in vitro and in vivo evaluation of the formulations 55, 64, and 96 from set-1 formulations is given in Figure 2-17. Formulation 64 showed at least 7 months' in vitro and in vivo LNG release with its in vitro depots maintaining integrity until the end of the study, and has shown low in vivo burst. The 55 formulation could last only for 3 months in vitro and in vivo. 96 formulation showed 5- 7 month LNG in vitro and in vivo release profile, with detectable LNG concentration in 3 rats at 7 months post injection, but has shown 5.5 times higher in vivo burst LNG levels than 64 formulation. This promising data indicated that a formulation in between 64 and 96 would probably achieve robust 6-7 month LNG release, with better injectability and low initial burst.

\section{Design of Formulation Set - 2}

The major difference in composition between the 64 and 96 formulations is PLA intrinsic viscosity ( 0.63 vs. 0.47$)$ or MW and amount (18.8\% vs. $12 \%)$, solvent type (NMP/TEC vs. NMP/BB) and LNG amount (6\% vs. 2.5\%). In order to improve the formulation release duration, injectability, and lower the burst following optimization strategies have been adopted: 


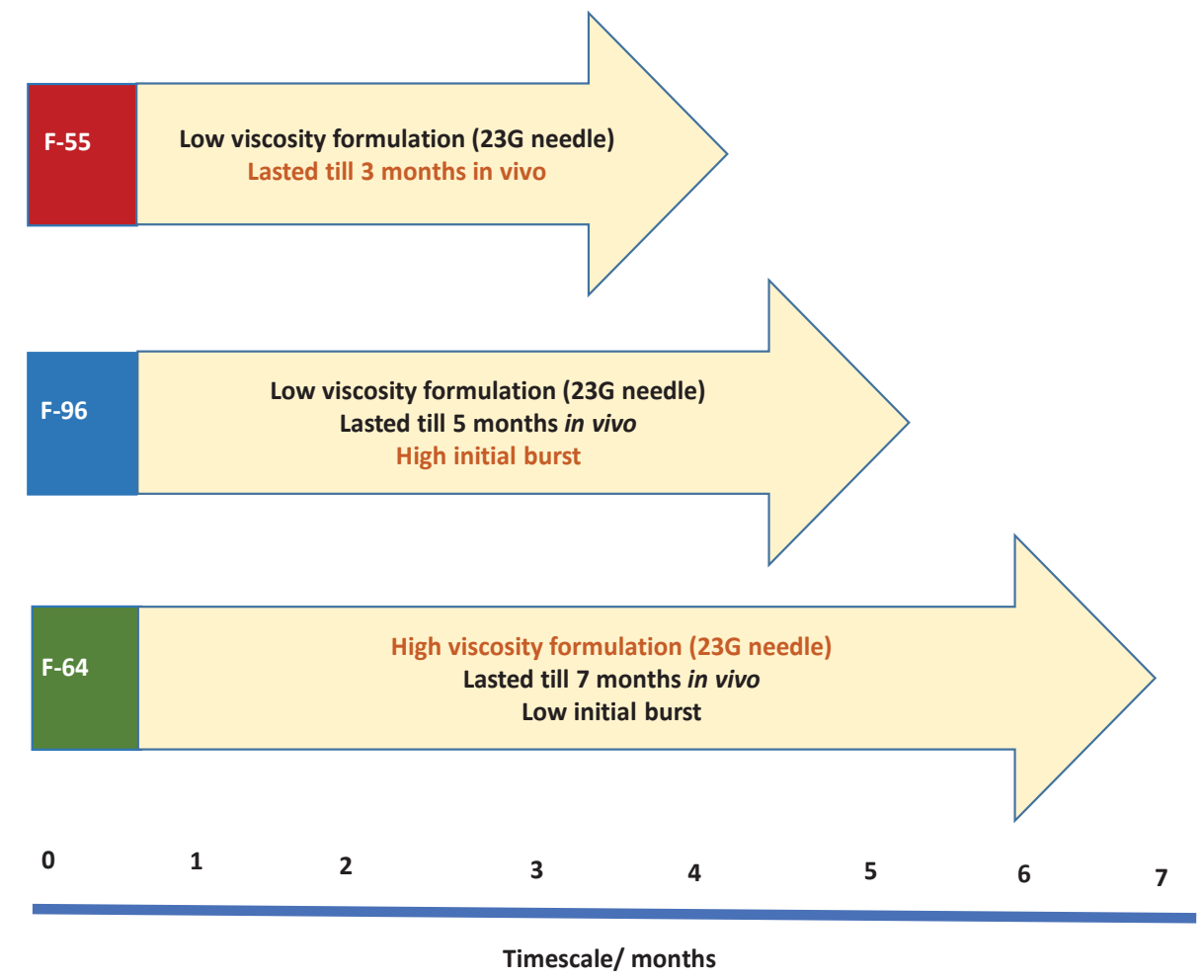

Figure 2-17. Key results summary of 55, 64 and 96 ISD formulations. 
- Started with the 96 formulation and replaced the PLA (iv 0.47) in the 96 formulation with PLA (iv 0.63, higher viscosity/MW, the polymer used in the 64 formulation) at 3 different weight amounts: $20 \%, 16 \%$, and $12 \%$.

- Replaced BB solvent in the 96 formulation with TEC (solvent used in the 64 formulation) or ethyl acetate (EA, studies showed that EA maintained good in vitro gel integrity and drug release profile [239])

- Increased the drug loading to 4\% (but maintained below 6\% to avoid any solubility issues).

- Kept the PLA polymer amount ( 20\%) as 64, but used a blend of PLA (iv 0.47 ) and PLA (iv 0.63).

\section{In Vitro Accelerated Release Study}

Conducting the real-time release under physiological conditions (PBS, pH 7.4) can take months to years depending on the required drug release duration (Figure 2-6), which is laborious and expensive. Therefore, a fast and reliable accelerated in vitro release method that can predict the real-time release in a short time is needed to save time and money [11,12]. Unfortunately, although several accelerated in vitro release methods have been developed for PLGA or PLA-based microspheres and preformed implants [12], but there is no accelerated in vitro release method for injectable ISD systems reported in the literature yet. Studies were conducted to develop an accelerated in vitro drug release method for LNG-loaded ISD systems for rapid screening/ differentiation/ optimization of long-acting contraceptives, and also provide some guidance that can be useful for the development of accelerated release methods for ISD systems in general.

The selection of appropriate stress conditions for accelerating the drug release is dependent on the nature and composition of the formulation, stability of the drug and release medium, release mechanisms, and requirements related to the sampling interval and duration of the study [14]. In this work, different combinations of four conditions of release media including: (a) temperature [11,14-27]; (b) presence of organic solvents such as alcohol, acetone, acetonitrile as co-solvents [12,15,28]; (c) presence of surfactants $[18,28-31]$, and (d) $\mathrm{pH}[12,15,16]$ were used to develop an accelerated in vitro drug release method for screening ISD systems for long-term release of LNG for contraception. The correlations between the release kinetics and mechanisms of LNG from ISD systems under real-time and accelerated conditions were established [240].

\section{Accelerated (short-term) in vitro release}

96 and 64 formulations were used as control formulations for developing the accelerated release condition. As the real-time in vitro LNG release took months (Figure 2-6) to be conducted, four different release conditions were explored as listed in Table 2-4 to develop an accelerated short-term ( 2 weeks) release method that would correlate well with the real time long term release so that it can be used for screening ISD systems for sustained release of LNG. The accelerated release profiles of the formulations 96 and 64 at four different conditions tested are shown in Figure 2-18I, II, respectively. 

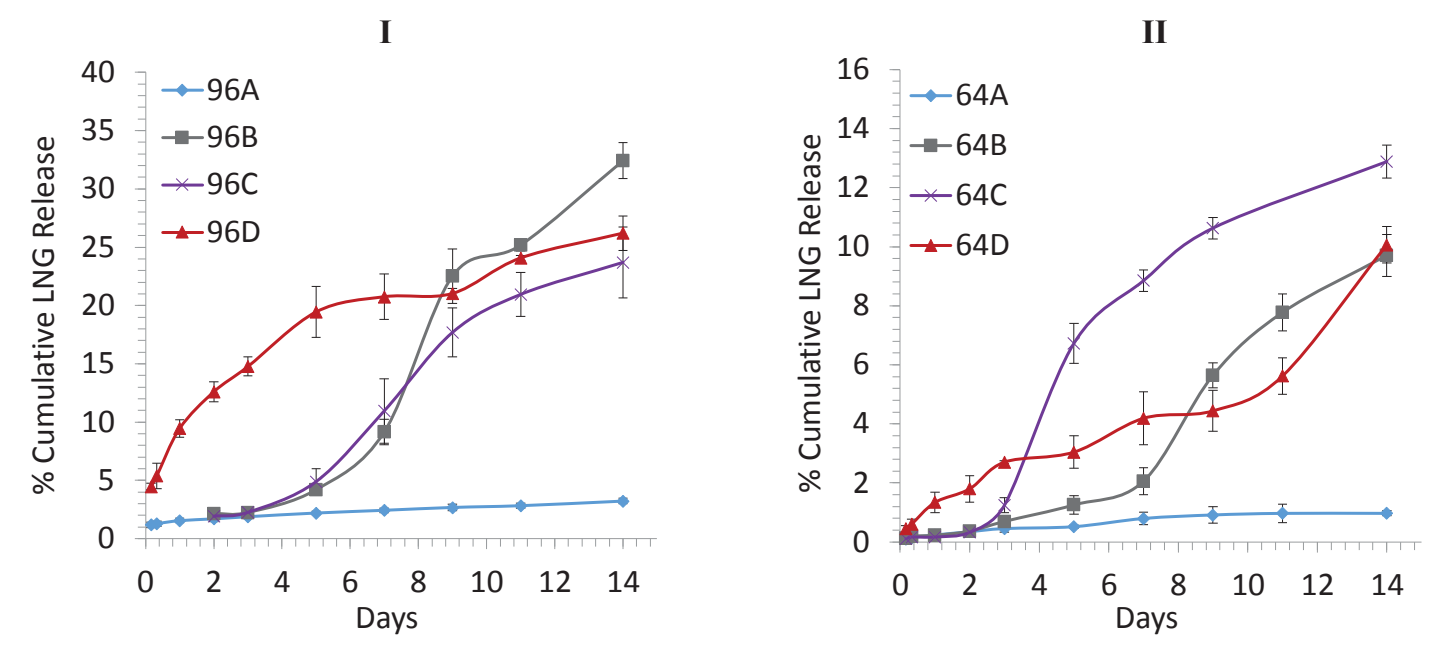

Figure 2-18. Short-term (accelerated) in vitro LNG release from formulations 96 (I) and 64 (II) at four accelerated conditions.

Notes: (A) PBS, $\mathrm{pH} 7.4,50{ }^{\circ} \mathrm{C}$ (filled diamond); (B) $25 \%$ ethanol in PBS, $\mathrm{pH} 7.4,50{ }^{\circ} \mathrm{C}$ (filled square); (C) $25 \%$ ethanol in PBS, $2 \%$ Tween $20, \mathrm{pH} 7.4,50{ }^{\circ} \mathrm{C}$ (cross mark); (D) $25 \%$ ethanol in PBS, $2 \%$ Tween $20, \mathrm{pH} 9,50{ }^{\circ} \mathrm{C}$ (filled triangle). Each point represents mean $\pm \mathrm{SD}, \mathrm{n}=3$ ). 
Under elevated temperature alone (condition A: PBS, pH 7.4, $50{ }^{\circ} \mathrm{C}$ ), Figure 2-18I, II show that only $3.2 \%$ and $0.97 \%$ of LNG was released from the formulations 96 and 64, respectively, during 14 days. These amounts were slightly higher than those corresponding amounts, $\sim 2.97 \%$ (96) and $\sim 0.47 \%$ (64), at $37^{\circ} \mathrm{C}$ (Figure 2-6). When the release was conducted at elevated temperature, the drug release rate would increase as the drug diffusion and polymer degradation rate increased with temperature [11,15-22,34]. However, at elevated temperature, the polymer's mobility also increased, which could cause a decrease in the drug release rate as the polymer movement could change the depot surface morphology and cause surface pore closure [11]. Possibly due to the competing effects mentioned above, the overall drug release rates from the two formulations did not increase too much when the temperature was increased from 37 to $50{ }^{\circ} \mathrm{C}$. Further investigation is needed to test this hypothesis.

To achieve a higher LNG release rate, the temperature could be further increased. However, to avoid any potential high temperature related stability concern of the drug, we set the release temperature at $50^{\circ} \mathrm{C}$ and explored the effects of additional parameters such as addition of organic solvent ethanol (condition B), and addition of ethanol and surfactant Tween 20 (condition $C$ ) in the release media on the drug release rate. Organic solvents such as alcohol, acetone, and acetonitrile added to the release medium as cosolvents were reported to accelerate drug release due to the swelling property of the hydro-alcoholic solvent which could cause the morphological changes such as surface pitting and pore formation of depots with time (hydro-alcoholic effect) [12,15,28]. As ethanol is more environmentally friendly than acetone and acetonitrile, we chose ethanol as a co-solvent for the accelerated release study. Surfactants such as Tween 20, Brij 35P, Triton X-100 could form micelles in the release medium to extract out the hydrophobic drug loaded in the depot so that the drug would be released faster from the depot $[28,35,36]$. In this accelerated release study, we chose ethanol as a co-solvent and Tween 20 as a surfactant. Figure 2-18I (96B),II (64B) showed that replacement of $25 \%$ of the release medium with ethanol did not cause much change in the LNG release rates from the two formulations 96 and 64 during the initial three days; the LNG release slowly increased during days 3 to 7 , and the LNG release increased significantly during days 7 to 14 and about 9-fold on day 14. These results suggested that the hydro-alcoholic effect did not work so well to accelerate LNG release during the first 3 days. To study if the further addition of surfactant would accelerate more LNG release, we added Tween 20 at $2 \mathrm{wt} \%$ in the release media and found that the addition of Tween 20 did not have much effect on the LNG release from formulations 96 and 64 during the first 7 and 5 days, respectively; decreased the LNG release from formulation 96 during 7-14 days, however, it increased the LNG release from formulation 64 dramatically after day 5 (Figure 2-18I(96C), II(64C)). The different effects of Tween 20 on LNG release from 96 and 64 formulations could be due to the differences in the porosities of the depots at condition $\mathrm{C}$, which led to variation in the amount of Tween 20 micelles formed in the polymer matrix. The depot formed from 96 formulation probably generated more and faster pores than that from 64 formulation (similar to the real-time conditions, Figure 2-7) as the corresponding inherent viscosities of the PLA used for the 96 and 64 depot preparation were 0.47 and $0.63 \mathrm{dL} / \mathrm{g}$, respectively. The more porous 96 depot may have allowed more surfactant to come inside the depot to form more micelles to hold more drug inside the depot. As a 
result, the addition of surfactant Tween 20 would have caused a significant amount of micelles formed inside the depot formed from 96 formulation so that LNG was released less from the depot under condition $\mathrm{C}$ than condition $\mathrm{B}$ after 7 days. The above interpretations of the results need further investigation.

As the three accelerated conditions $\mathrm{A}, \mathrm{B}$ and $\mathrm{C}$ were unable to cause fast release during the first three days, then the $\mathrm{pH}$ of the release medium was increased from 7 to 9 (condition D). Figure 2-18I, II show that the release of LNG from both the 96 and 64 formulations was significantly increased during the first three days under condition $\mathrm{D}$ in comparison to the other three conditions $\mathrm{A}, \mathrm{B}$, and $\mathrm{C}$. The reason was probably due to the faster degradation of PLGA/PLA in the depot caused by alkaline $\mathrm{pH}$ 9, which was already well documented in the literature [37,38]. After 3 days, LNG was continuously released under condition $\mathrm{D}$ with amount about 8-10 times on day 14 than under condition A ( $\mathrm{pH} 7.4)$; and higher and lower under condition D than condition $\mathrm{C}(\mathrm{pH} 7.4,25 \%$ ethanol, 2\% Tween 20) after three days for formulations 96 and 64, respectively. These results were probably due to the balance of the polymer degradation and micelle formation inside and outside the depots.

The long-term goal of this study was to develop injectable formulations for sustained release of contraceptives for five months to years. Due to the intended long term release, the real-time 4-6 months in vitro release in Figure 2-6 and the accelerated 2-week release in Figure 2-18 did not reach 80\% LNG release, but instead reached $<10 \%$ and $<35 \%$, respectively. According to FDA recommendation, an in vitro release should be conducted until the time point when $80 \%$ or higher or a plateau of drug release is attained [39]. However, the objective of the study was to identify an accelerated release condition that could be used to differentiate formulations during early formulation screening stage quickly rather than to develop a standard in vitro release protocol. The identified accelerated release condition $\mathrm{D}$ for a period of 2 -week release study was sufficient and effective to achieve this objective.

\section{Release kinetics and correlation between accelerated and real-time releases}

To understand the release mechanisms of LNG from formulations 96 and 64 in real-time and accelerated conditions, all the release data in Figure 2-18 were fitted using empirical power law (Korsmeyer-Peppas Model, Equation 2-5) [40]. Table 2-15 lists the parameters $k, n$ and coefficient of determination $\left(r^{2}\right)$ after fitting the release curves of 96 and 64 formulations in Figures 2-6 and 2-18 using Equation 2-5.

The release curves in Figures 2-6 and 2-18 could be fitted well by Equation 2-5 with $r^{2} \geq 0.94$ except for 64B and 64C which had slightly lower $r^{2}$ values of 0.86 and 0.88 , respectively. The $n$ values ranged from 0.22 to 1.59 suggesting different release mechanisms under the four accelerated conditions for the formulations 96 and 64 . The conditions A, B, and C either slightly increased (96A, 64A, 64B, and 64C) or decreased (96B and 96C) the release rate constant $k$. The condition D significantly increased the $k$ values by about $\sim 10.2$ and $\sim 5.8$ times for formulations 96 and 64, respectively, but well mimicked the release kinetics under real time condition for the two formulations with the 
Table 2-15. Fitting parameters determined by the linear regression of $\log \left(M_{t} / M_{\infty}\right)$ against $\log t$ in Equation $2-5$ for the LNG release from the depots made of formulations 96 and 64 under real time and four accelerated release conditions.

\begin{tabular}{lccc}
\hline $\begin{array}{l}\text { Formulation } \\
\text { (Release } \\
\text { condition) }\end{array}$ & \multicolumn{2}{c}{ Power law (Korsmeyer-Peppas model) } \\
\hline \cline { 2 - 4 } 96(A) & $\begin{array}{r}\boldsymbol{r}^{2} \text { (Coefficient of } \\
\text { determination) }\end{array}$ & $\boldsymbol{k}$ (Rate constant) & $\begin{array}{c}\boldsymbol{n} \text { (Release } \\
\text { exponent) }\end{array}$ \\
96(B) & 0.96 & 1.62 & 0.22 \\
96(C) & 0.94 & 0.50 & 1.59 \\
96(D) & 0.97 & 0.57 & 1.15 \\
96 (long-term) & 0.99 & 9.23 & 0.41 \\
64(A) & 0.99 & 0.90 & 0.46 \\
64(B) & 0.99 & 0.26 & 0.52 \\
64(C) & 0.86 & 0.41 & 0.97 \\
64(D) & 0.88 & 0.49 & 1.24 \\
64 (long-term) & 0.97 & 1.28 & 0.64 \\
\hline & 0.97 & 0.22 & 0.65 \\
\hline
\end{tabular}


similar fitting linearity ( $r^{2}=0.99$ for both $96 \mathrm{D}$ and $96 ; r^{2}=0.97$ for both 64D and 64) and release mechanisms ( $n=0.41$ and 0.46 for $96 \mathrm{D}$ and 96 , respectively; $n=0.64$ and 0.65 for 64D and 64, respectively). These results are visually illustrated in Figure 2-19 (I, II).

To further correlate the short- and long-term LNG releases, the times required to achieve the same amounts of LNG to be released from the formulations 96 and 64 under condition D (days) and real-time (months) are shown in Figure 2-20 [26,34]. Excellent correlations between the release times under condition $\mathrm{D}$ and real-time were obtained with $r^{2}=0.98$ and 0.99 for formulation 96 and 64, respectively. These results suggested that the condition $\mathrm{D}$ was able to reduce the release time significantly compared to the real-time, and allows for the prediction of LNG long-term release from ISD systems in a short period. Therefore, condition $\mathrm{D}$ was used to screen the set-2 formulations for initial in vitro release screening.

\section{Screening of Set-2 ISD Formulations}

Various formulation variables were investigated to reduce the undesired burst. In each experiment, the standard formulations 96 and 64 were repeated as a reference for comparing viscosity (injectability) and initial drug release, respectively. Initially the formulations (96j-96o) were screened for shear viscosity and the initial release in 24 hours at accelerated condition $\mathrm{D}$. The results showed that lowering the polymer concentration from $24 \%(96 \mathrm{j}, 96 \mathrm{~m})$ to $20 \%(96 \mathrm{k}, 96 \mathrm{n})$ to $16 \%(961,96 \mathrm{o})$ has resulted in a significant decrease (at least 2 fold) in the viscosity (Figure 2-21A) of the formulations. However, lowering the polymer percentage has led to significant increase in the initial release compared to the 64 formulation (Figure 2-21B). Thus, the polymer concentration played a significant role in the ISD formulations. A higher polymer concentration led to a more viscous solution, which might have delayed the polymer precipitation and resulted in a less porous polymer matrix with a slower drug release [241]. On the other hand, increasing the drug loading from $2.5 \%(96 \mathrm{j} / 96 \mathrm{k} / 961)$ to $4 \%(96 \mathrm{~m} / 96 \mathrm{n} / 96 \mathrm{o})$ lead to increase in the viscosity $(\sim 1.2$ to 2 fold $)$ and a decline of the injectability of the formulations, and the extent of increase is more prominent in case of the formulations that had high polymer percentage (96j vs. 96m). At low polymer percentages, the drug release rate was increased with increased drug loading which was consistent with the literature [194]. However, in the case of formulations with high polymer percentage $(24 \%)$, increased loading has resulted in decreased initial release rate. This opposite pattern could be attributed to significantly increased viscosity ( $\sim 2$ fold $)$ of the $96 \mathrm{~m}(4 \%$ LNG) formulation compared to the $96 \mathrm{j}(2.4 \% \mathrm{LNG})$, which might have decreased the rate of phase inversion dynamics and slowed the initial release [241].

It was observed that the formulations that showed promising release profile as 64 are $96 \mathrm{j}$ and $96 \mathrm{~m}$. This suggested that $24 \%$ of the polymer may be needed to get the desired slow release profile as 64 formulation in vivo. However, injectability at such high polymer percentage is a drawback. Therefore, keeping the polymer percentage $(24 \%)$ and PLA amount (20\%) constant, the higher inherent viscosity PLA (0.63 iv; $103 \mathrm{kDa} \mathrm{MW})$ was substituted with a lower inherent viscosity PLA $(0.47 i v ; 67 \mathrm{kDa} \mathrm{MW})$ at various proportions to design 96p, 96q, and 96r formulations. Higher drug loading is favored in 

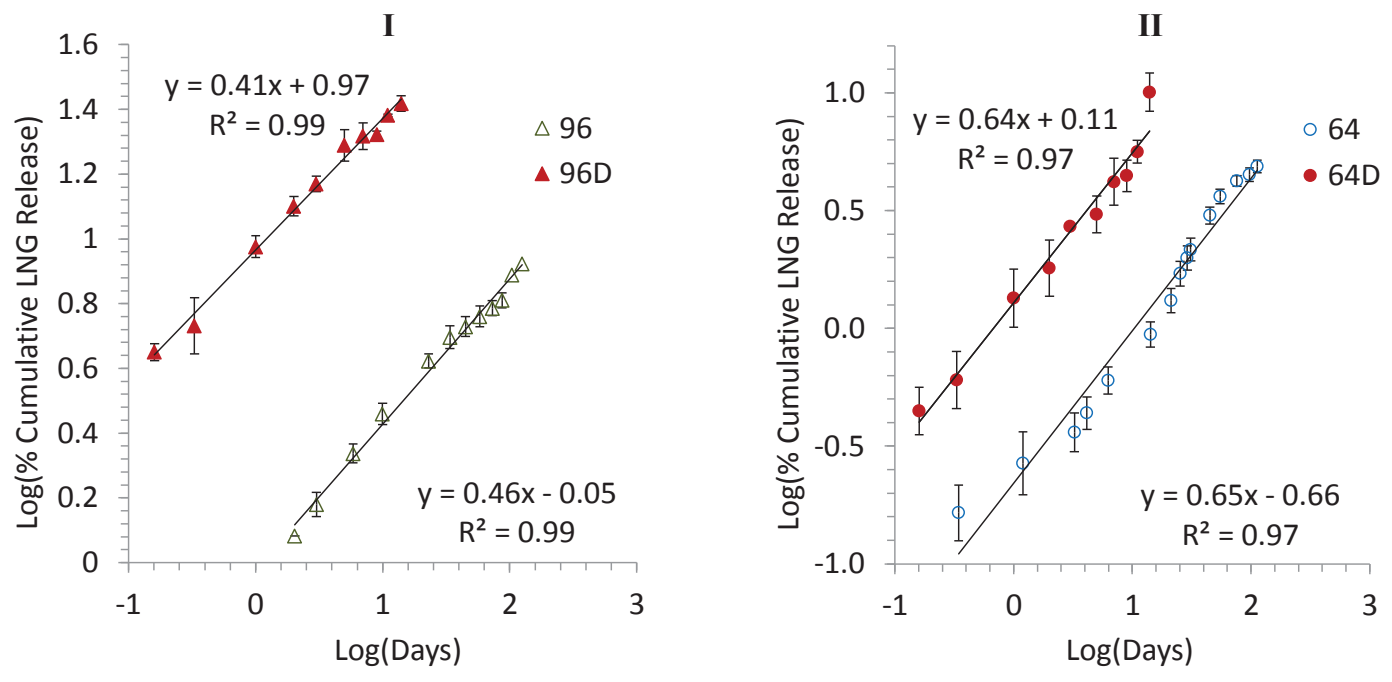

Figure 2-19. Power law kinetics (plot of log percent release vs. log of time in days) of formulations.

Notes: 96 (I; triangle) and 64 (II; circle) in real-time (open symbols) and accelerated (filled symbols) release condition $\mathrm{D}$. Each point represents mean $\pm \mathrm{SD}, \mathrm{n}=3$ 
I

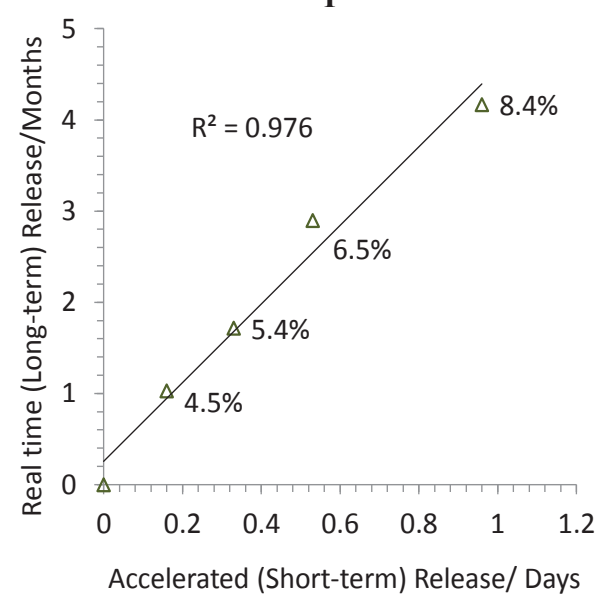

II

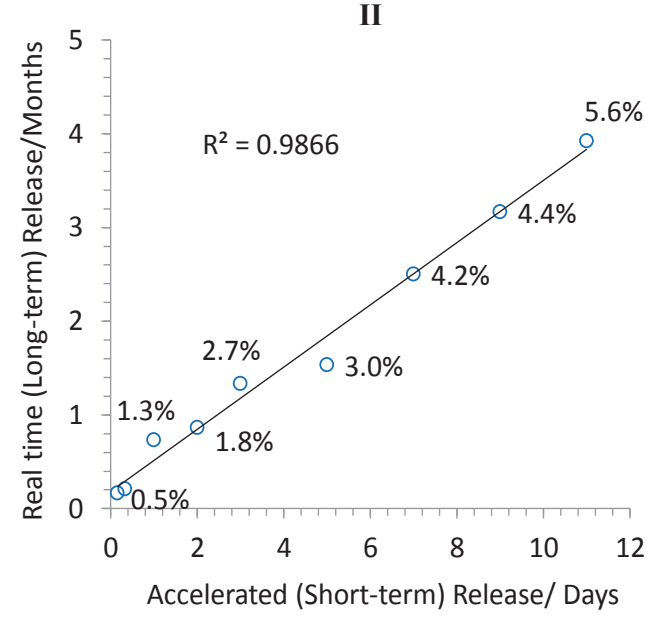

Figure 2-20. Correlation of accelerated (short-term) release at condition $D$ with real-time (long-term) release testing of formulations 96 (I; triangle) and 64(II; circle). 

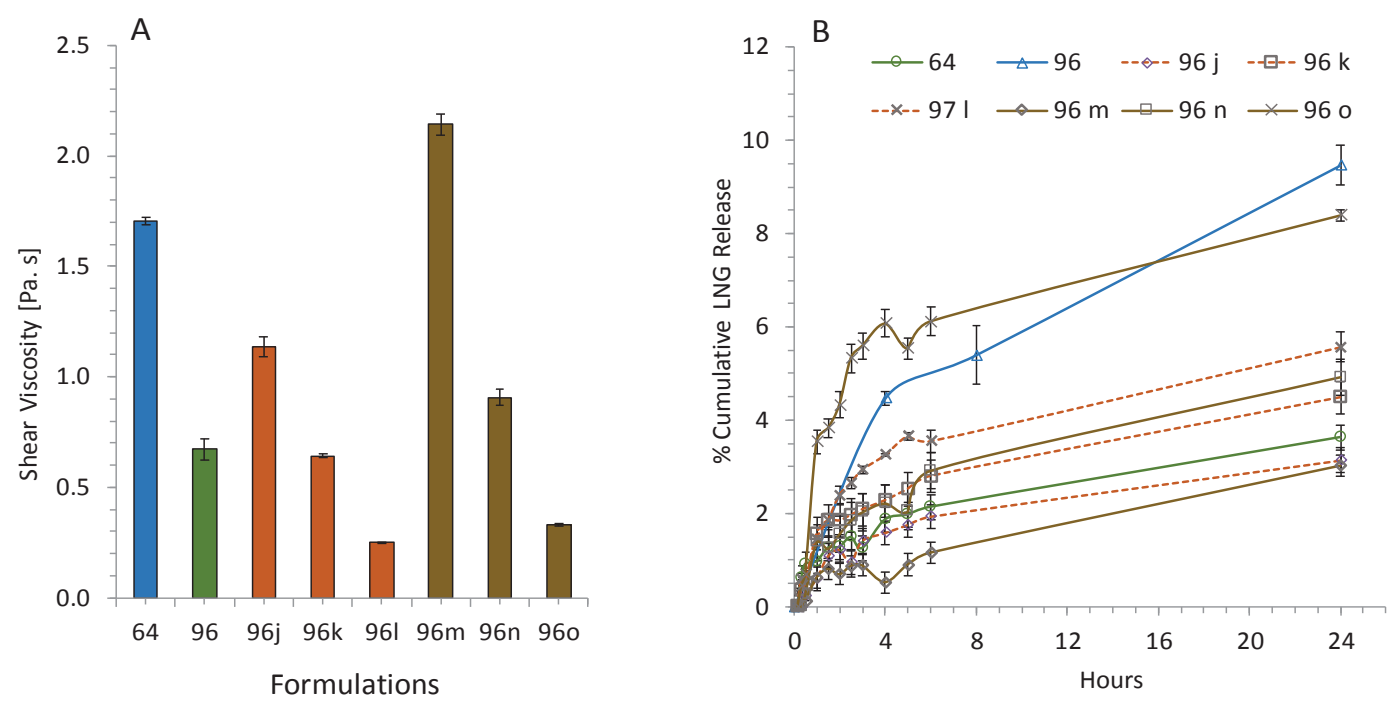

Figure 2-21. Shear viscosity (A) and LNG in vitro release in accelerated condition D (B) of different ISD formulations.

Notes: Polymer percentage: $24 \%$ in $(96 \mathrm{j}, 96 \mathrm{~m}) ; 20 \%$ in $(96 \mathrm{k}, 96 \mathrm{n}), 16 \%$ in $(961,960)$. Complete composition listed in Table 2-3. Each point represents the mean $\pm \mathrm{SD}, \mathrm{n}=3$. 
terms of injection volume, as it would result in a smaller injection volume at the same dose. Therefore, $4 \%$ LNG was loaded into the formulations instead of $2.5 \%$ and tested for the shear viscosity. Figure 2-22 shows the shear viscosities of the 96p, 96q, and 96r formulations. The $96 \mathrm{r}$ formulation has a viscosity of $(0.75 \pm 0.02 \mathrm{~Pa} . \mathrm{s})$, similar to that of 96 formulation $(0.65 \pm 0.05 \mathrm{~Pa} . \mathrm{s})$ and can be easily injected through 23 gauge needle.

Another formulation 96zz was prepared by replacing the TEC in 96r formulation with ethyl acetate. The rationale for designing $96 \mathrm{zz}$ is that EA could increase not only the hydrophobicity of the preparation but also decrease the viscosity. Further, earlier studies from our group showed that EA maintained good in vitro gel integrity and drug release profile [239]. Replacement of TEC with EA has resulted in a decrease in the mean viscosity of the formulation from 0.75 to $0.66 \mathrm{~Pa}$.s. Apart from the low viscosity, another desirable attribute of these 96r and $96 \mathrm{zz}$ formulations is that both formulations showed slower release rate of LNG than 64 formulation during the initial accelerated in vitro release study (Figure 2-23). The order of in vitro LNG release rate during the initial 4 hours was $96 \mathrm{zz}<96 \mathrm{r}<64$.

Both 96r and 96zz formulations which have shown injectability through the $23 \mathrm{G}$ needle and promising in vitro release profile were selected for in vivo burst study and injected SubQ into the rats at a dose of $10 \mathrm{mg}$ LNG. Figure 2-24 shows the comparison of plasma LNG concentration as a function of time in first $24 \mathrm{~h}$ after injecting the formulation $96 \mathrm{r}, 96 \mathrm{zz}$, and 64 . The $C_{\max }, t_{\max }$, and $A U C_{(0-24 h)}$ of $96 \mathrm{r}$ and $96 \mathrm{zz}$ were estimated and given in Table 2-16. The $C_{\max }$ and $A U C_{(0-24 h)}$ of LNG for the formulations were in the order of $64<96 \mathrm{r}<96 \mathrm{zz}$. The initial release of LNG from 96r, 96zz and $64 \mathrm{in}$ vivo was opposite to that observed in vitro. This difference could be attributed to the differences in the in vitro and in vivo conditions and the physiochemical characteristics of the formulations. During in vitro studies, as the formulation was injected into the mold and placed in the jar containing release medium, no external forces were acting on the depot. On the other hand, when the ISD formulation was injected in vivo, a high amount of shearing forces from the surround tissue environment will be acting on the depot. These surround forces could exert a squeezing on the depot expelling the solvent quickly in comparison to the in vitro depots which experience no external forces. Another factor could be related to the viscosity of the formulations. The $C_{\max }$ of the formulations was increased with a decrease in the viscosity of the formulation, which could be due to higher rate of phase inversion dynamics with decreased viscosity leading to faster initial release [241]. Although 96r and 96zz formulations have similar composition except for co-solvent (TEC vs EA), a significant difference ( 2.7 fold) in the $C_{\max }$ was observed between 96r and 96zz. EA has more 1.44 times higher water miscibility and 77.9 fold lower viscosity than TEC. Because of the high water miscibility (1.4 fold) and significant low viscosity (77.9 fold) of the co-solvent EA compared to TEC, the solvent mixture NMP:EA (96zz) might have left the system rapidly compared to the NMP:TEC (96r) mixture during in vivo conditions because of tissue compressive forces. This fast diffusion of the solvent along with dissolved drug might have resulted in the higher initial release of LNG in vivo for 96zz formulation compared to 96r. The 96r formulation has shown promising in vivo profile close to 64 formulation. To this end, the optimized formulation $96 \mathrm{r}$ has favorable attributes which include injectability through a $23 \mathrm{G}$ needle 


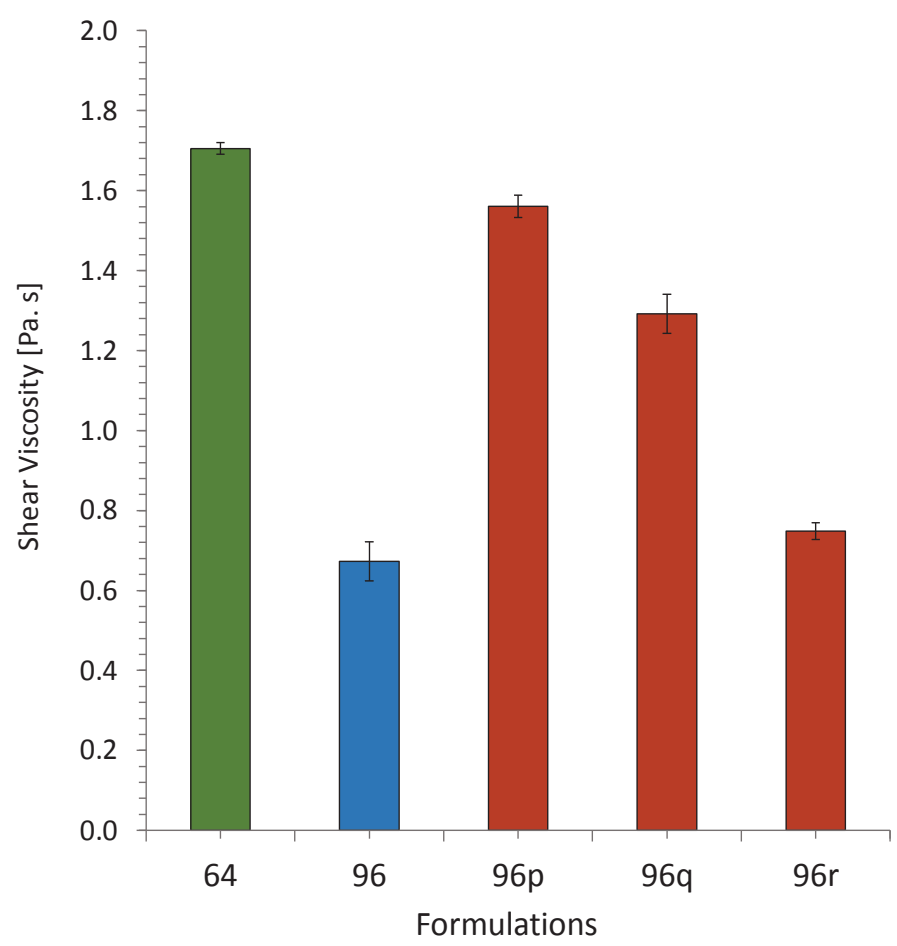

Figure 2-22. Shear viscosities of 64, 96, 96p, 96q, and 96r formulations.

Notes: LNG Loading and polymer percentage in formulations (96p, 96q, and 96r) is 4\% and $24 \%$, respectively. However, the formulations varied in the polymer composition. Complete composition listed in Table 2-3. Each point represents the mean $\pm S D, n=3$. 

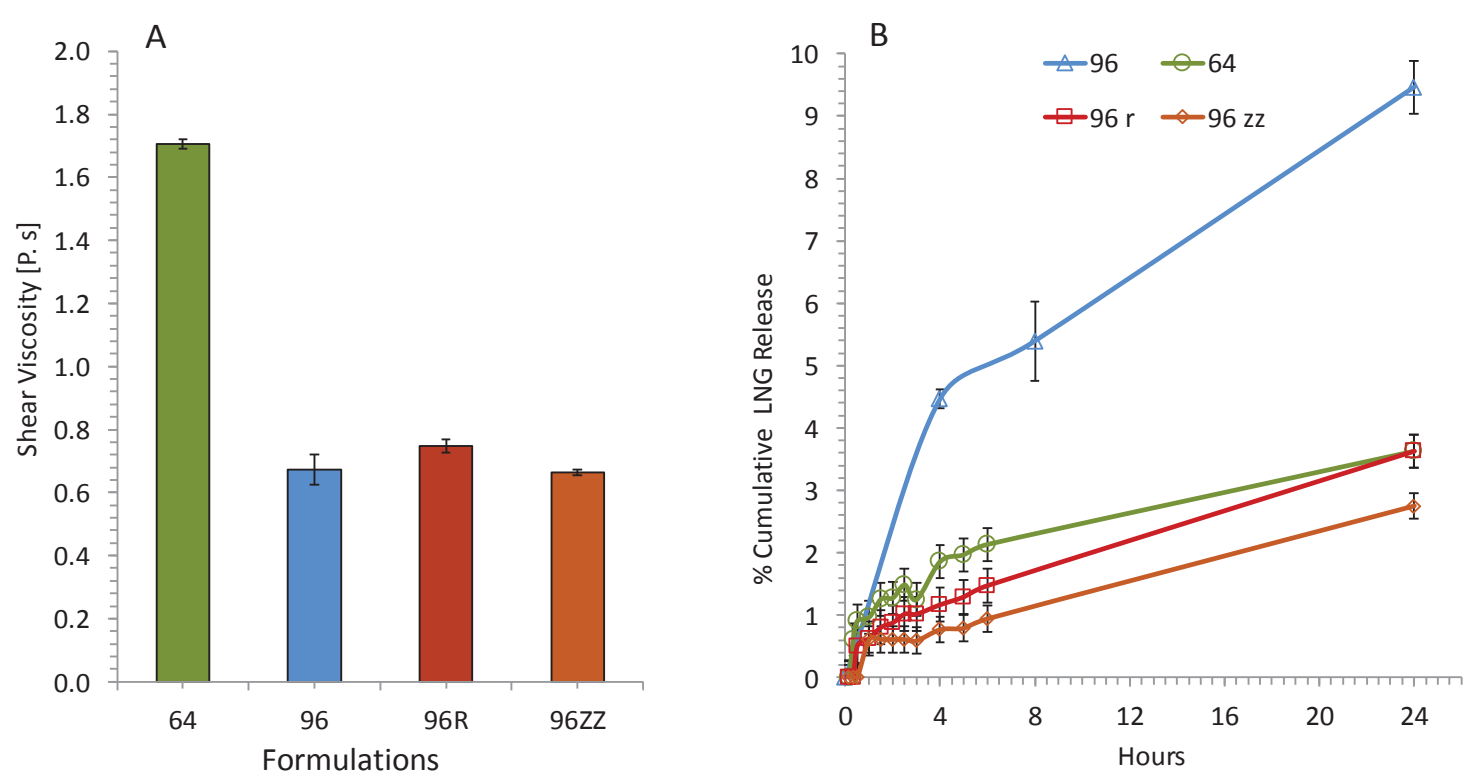

Figure 2-23. Shear viscosity (A) and LNG in vitro release in accelerated condition $D$ (B) of the formulations $64,96,96 \mathrm{r}$ and $96 \mathrm{j}$.

Notes: The difference between $96 \mathrm{r}$ and $96 \mathrm{zz}$ is the co-solvent (TEC vs EA). Complete composition listed in Table 2-3. Each point represents the mean $\pm \mathrm{SD}, \mathrm{n}=3$. 


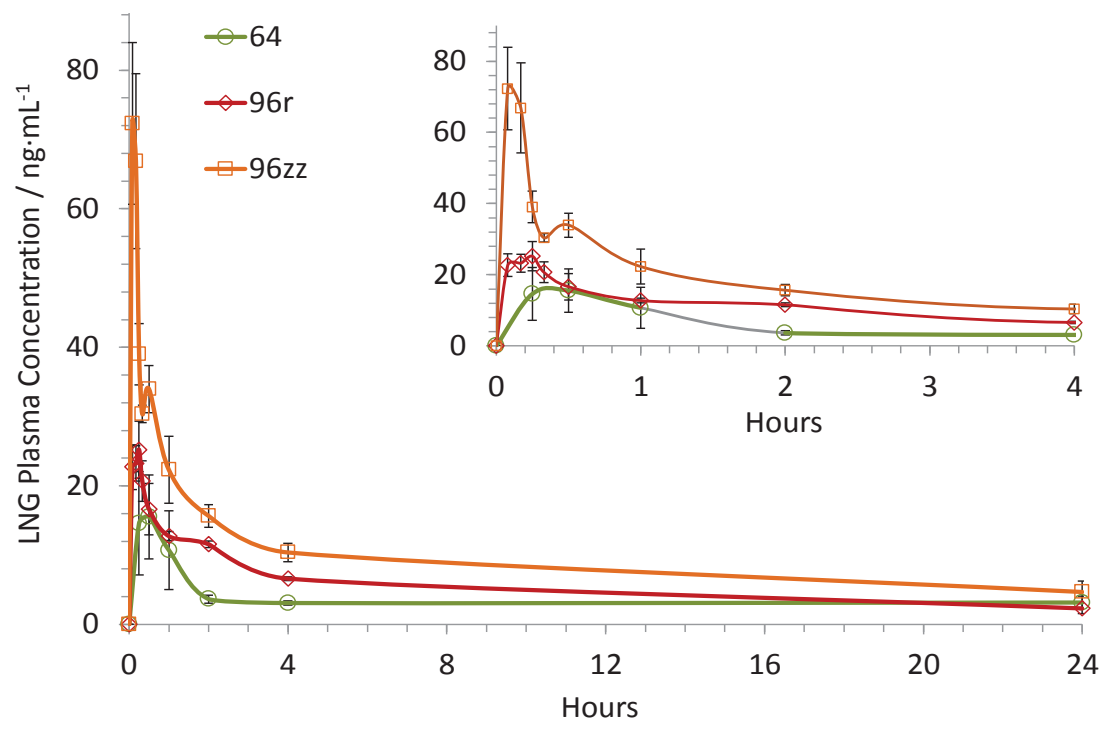

Figure 2-24. LNG Plasma concentration measured in rats as a function of time after SubQ injection of 64, 96r, 96zz formulations.

Notes: Dose: $40 \mathrm{mg} \cdot \mathrm{kg}^{-1}$. The difference between $96 \mathrm{r}$ and $96 \mathrm{zz}$ is the co-solvent (TEC vs EA). Each point represents the mean \pm SE, $n=3-5$. 
Table 2-16. Pharmacokinetic parameters of 96r and 96zz formulations in rat plasma after 24 hours post SubQ injection.

\begin{tabular}{|c|c|c|c|c|}
\hline $\begin{array}{c}\text { Formulation } \\
\#\end{array}$ & $\begin{array}{c}\text { Injection dose } \\
\mathrm{mg} \cdot \mathrm{Kg}^{-1}\end{array}$ & $\begin{array}{c}C_{\max } \\
\mathbf{n g} \cdot \mathbf{m L}^{-1}\end{array}$ & $\begin{array}{c}T_{\max } \\
\mathbf{h}\end{array}$ & $\begin{array}{c}A U C_{(0-24 h)} \\
\mathrm{ng} \cdot \mathbf{h r} \cdot \mathrm{mL}^{-1}\end{array}$ \\
\hline $96 r(n=3)$ & 40 & $29.0 \pm 6.39$ & $0.20 \pm 0.02$ & $149.14 \pm 12.19$ \\
\hline $96 z z(n=5)$ & 40 & $79.0 \pm 11.1$ & $0.25 \pm 0.08$ & $231.22 \pm 33.07$ \\
\hline
\end{tabular}


(close to 96 formulation), and slow in vitro release, and low initial in vivo burst (close to 64 formulations). The lead formulation $96 \mathrm{r}$ has great potential to show a long-term in vivo $\mathrm{LNG}$ release.

\section{Conclusions}

A series of LNG-containing ISD formulations were designed by employing unique strategies which include the use PLGA, PLA with different biodegradable properties, and blends of these polyesters, use solvent mixtures of NMP, TEC, BB, EA and with varying polymer/solvent ratios, and drug loadings. This study has investigated and effect of formulation variables on injectability, in vitro release and in vivo initial burst of LNG-containing ISD systems.

In the process, new methods have been developed and reported for detecting LNG in rat plasma, and for accelerated in vitro release of LNG from ISD formulations for rapid screening. The details and the finding from these two new methods are:

- A simple and sensitive LC/MS/MS method for quantitative bioanalysis of levonorgestrel in rat plasma was developed and validated and applied to pharmacokinetics study of LNG in rats. LNG was successfully extracted and quantified in rat plasma using the presently developed LC/MS/MS method, and the resultant mass spectra was satisfactory in terms of identification, least interference from matrix components and better quantification. The developed liquid-liquid extraction method using hexane/ethyl acetate $(80 / 20, \mathrm{v} / \mathrm{v})$ mixture was fast and efficient in terms of matrix effect and recovery efficiency. Moreover, this technique required a small amount of rat plasma $(100 \mu \mathrm{L})$ unlike any previously reported methods which used $0.5-1 \mathrm{~mL}$ of human plasma. Calibration curve was successfully constructed within a concentration range of $0.5-50 \mathrm{ng} \cdot \mathrm{mL}^{-}$ ${ }^{1}$. The developed method was successfully validated for specificity, accuracy, precision and stability. Furthermore, this validated method was successfully applied to study LNG pharmacokinetics after single SubQ injection of LNG alone and LNG-containing ISD formulations in rats.

- An accelerated release study to evaluate long-acting contraceptive LNGcontaining ISD systems was developed. In summary, elevated temperature, addition of alcohol and surfactant, and change of the $\mathrm{pH}$ of the releasing medium to basic could result in an increase in the drug release rate of ISD systems, due to higher degradation of the depot polymers, increase of the porosity of the depot matrices, and thus fast diffusion of the drug out of the depots. The developed accelerated release condition D (25\% ethanol in PBS, $2 \%$ Tween $\left.20, \mathrm{pH} 9,50{ }^{\circ} \mathrm{C}\right)$ shortened the 4-5 month real-term LNG release from formulations 96 and 64 in PBS (pH 7.4) at $37^{\circ} \mathrm{C}$ to days without changing the drug release mechanisms. The developed accelerated release method has significance not only in helping rapid screening of formulations for the development of ISD-based contraceptives containing LNG, but also for providing guidance for the development of accelerated in vitro release testing methods for ISD systems in general. 
The summary of the key findings during the screening and optimization of ISD formulations for contraception that were outlined in this chapter:

- During the initial screening, the formulations 64 and 96 suppressed the rat normal estrous cycles for 5 and $\geq 7$ months, respectively after one SubQ injection. No significant swelling or inflammation was observed at the site of injection. After the end of the treatment, a rapid return of fertility was observed in rats. The prototype injectable ISD formulations (64 and 96) studied for long-acting contraception exceeded the current commercial products Depo-Provera ${ }^{\circledR} /$ DeposubQ Provera $104^{\circledR}$ in terms of duration ( $\geq 5$ vs. 3 months), and Eligard ${ }^{\circledR}$ in terms of needle size (21-23 vs. 18-20 G). However, 96 formulation with promising injectability showed significantly higher burst compared to 64 formulation. Therefore, the optimization process was further continued to get a balance between injectability and initial burst.

- The shear viscosity and injectability of the formulations was found to be proportional the polymer concentration, polymer inherent viscosity (or MW), and drug loading. In vitro LNG release studies demonstrated that polymer concentration and polymer inherent viscosity were the critical variables controlling the in vitro release. Decrease in polymer concentration (from $24 \%$ to $14 \%$ ), or polymer inherent viscosity (from 0.63 to 0.47 ) in formulations with similar composition has resulted in a decrease in shear viscosity ( 3 to 7 fold), increased injectability ( $23 \mathrm{G}$ needle), but showed faster in vitro LNG release.

- Formulations with TEC as secondary solvent showed a low initial burst in vivo compared to the similar formulations EA as co-solvent.

- The initial in vivo burst of LNG release was found to vary as a function of polymer concentration, polymer inherent viscosity, and co-solvent used in the ISD formulation.

- The formulations with the optimum balance between the injectability (23 G needle) and the LNG release was obtained with a total polymer concentration of $24 \%$, a solvent combination of NMP/TEC and a shear viscosity between 0.7-1 Pa.s. The optimized lead formulation 96r designed by a unique blend of PLGA and PLA polymers with different molecular weights has shown favorable injectability (23G) and in vivo initial burst, and has a high chance that it can show a promising long-term in vivo LNG release. The optimized formulation will serve as better candidates in terms of injectability and burst release and can be further investigated for long-term release and stability studies.

In conclusion, the results have demonstrated that the injectable ISD formulations can serve as an effective means of administering LNG with minimal tissue toxicity for long-term contraception suggesting that the designed ISD formulations have great potential for developing future robust and affordable injectable LARC products that could improve access to family planning around the globe. 


\section{Future Work}

There is a high possibility that the optimized formulation (96r) could show a promising long-term in vivo LNG release. Therefore, the future work of this project include: i) conducting long-term in vivo PK studies with the $96 \mathrm{r}$ formulation in rats to evaluate the extended release profiles of the LNG for five months, vaginal cytology, fertility study and in vivo safety, ii) continuation of the formulation optimization iii) developing a protocol for the terminal sterilization of the formulations, iv) evaluation of the stability of lead formulations upon storage, v) scale-up of lead formulations 


\section{CHAPTER 3. POLYSACCHARIDE-BASED NANOPARTICLES FOR DRUG DELIVERY ACROSS OCULAR/BLOOD-BRAIN BARRIERS}

\section{Background}

Since past few decades, several new chemical entities have been discovered as potent therapeutics for treating various diseases. In spite of the potency, more often their efficacy is still hampered by the inability to reach the target site of action $[35,36]$. These hurdles for the effective therapy of drug molecules (small or biologicals) could be attributed to the physicochemical properties of the drug molecules and the various barriers imposed by the body [242]. The human body possess various external (e.g. skin, intestine) and internal (bloodstream, the cytoplasm of tissue) physiological barriers which constitute the interface between different physiological environments so that a selective compartmentation is made for the effective regulation of distinct physiological functions in the body [242]. These act as a boundary between inside and outside of units such as a cell (e.g. cell membrane) or organ (e.g., cornea of the eye, BBB of brain) and usually permit the entry of oxygen and some key solutes, maintain water homeostasis and restrict the entry of other components and foreign materials [243]. They control the impact of external environment on the human body [35, 242]

Although these barriers are body's natural defense mechanism against foreign invaders, unfortunately, they pose significant hurdles to the drug therapy. Drugs need to traverse several physiological barriers to reach the target site to achieve effective drug concentration levels [36]. For instance, skin is the biggest obstruction to the topical drug delivery, while an epithelial layer of the GIT blocks the entry of drugs given orally. Even when administered via the parenteral route, drugs still need to overcome many barriers, either in blood or at the site of action. For example, the BBB and blood-retinal barrier (BRB) are the tightest barriers which prevent the entry of drugs from the bloodstream into the central nervous system and internal ocular tissues, respectively [243, 244]. In addition to the physiological (physical) barriers, another category of biological barriers that impede the drug activity are the biochemical and chemical barriers such as enzymatic degradation and elimination, the presence of efflux transporters [245-247].

For a drug to show its therapeutic activity, it is essential to overcome the physiological barriers and achieve desired concentrations at the target site [36]. These barriers may comprise of a single (e.g., intestinal epithelium) or multiple (e.g., skin, cornea) layers of cells that regulate the nutrients, electrolytes, and water exchange while restricting the entry of microbes, toxins, and antigens. The epithelial or endothelial cells which constitute the barrier have unique elements that promote barrier function, including apical-basolateral polarity and intracellular tight junctions. Two common pathways by which drugs cross these cellular layers are transcellular (through the cells), paracellular (between the cells) pathways. Transcellular drug transport is referred to the transportation of the drug across the membrane through penetration through the cell layer. The main mechanisms by which drugs penetrate through the cell layer are: (a) Passive diffusion represents the primary mechanism where the drug molecules from outside of the cell 
surface diffuse into the cells through the cell membrane spontaneously because of the concentration gradient; (b) Carrier-mediated diffusion - is the movement of molecules across the cell membrane with the help of some special transmembrane carrier molecules (or transport proteins) that are embedded in the cell membrane. This process can take place with (facilitated diffusion) or without (active transport) the need for energy consumption; (c) Vesicular transport: is a transcellular transportation mechanism in which the cell membrane frames a small pit that gradually encloses extracellular material to form a vesicle and then internalize them into the cell. The process is called endocytosis when the material is taken into the cell, exocytosis (reverse of endocytosis) when the material is exited out of the cell, and transcytosis when the material is transported across the cell. Paracellular drug transport is referred to the transportation of the drug across the membrane/barrier through the junctions between the cells. This process can be initiated by concentration (passive diffusion) or hydrostatic pressure (filtration) gradient across the layer. Paracellular transport between epithelial/endothelial cells is regulated by structures at cell-cell contact sites known as tight junctions [248]. All these transport mechanisms are regulated, different substances move using various aspects of trans/paracellular routes. Each of these pathways can be targeted to alter barrier function and enhance the drug permeability [249].

Some of the important physiological barriers that pose hurdles to drug transport are the intestinal epithelium, skin, ocular barriers, and blood-brain barrier. The barriers of interest to this project (ocular and blood-brain barriers) are discussed in detail below:

\section{Ocular Barriers}

The structure of the eye and ocular barriers for drug delivery is illustrated in Figure 3-1. Clinically, the anterior segment diseases/disorders are more often treated by using eye drops, or ointments, while the posterior eye segment diseases are treated by eye drops, or intravitreal injection/implantation. Local therapy via topical, periocular or intravitreal administrations is more favorable over the therapy via systemic administration such as oral and intravenous administrations to manage ocular diseases. The first reason is that the eye has much less blood vessels and blood flow than the whole body blood circulatory system so that the amount and rate of drugs to be cleared through local eye administration is much less than through systemic administration. The second reason is due to the presence of blood-aqueous barrier (BAB), and BRB which limits the drug penetration from the systemic circulation into the anterior and posterior segment of the eye, respectively.

\section{Routes of Administration and Ocular Barriers}

Several routes of administration for drugs to treat eye diseases include topical, intracameral, periocular routes (such as subconjunctival, sub-Tenon's, retrobulbar), intravitreal and systemic routes. Depending on the routes of administration, one or more ocular barriers need to be circumvented for drugs to reach the disease/action sites in the eye. Table 3-1 lists the routes of administration and their associated advantages and 


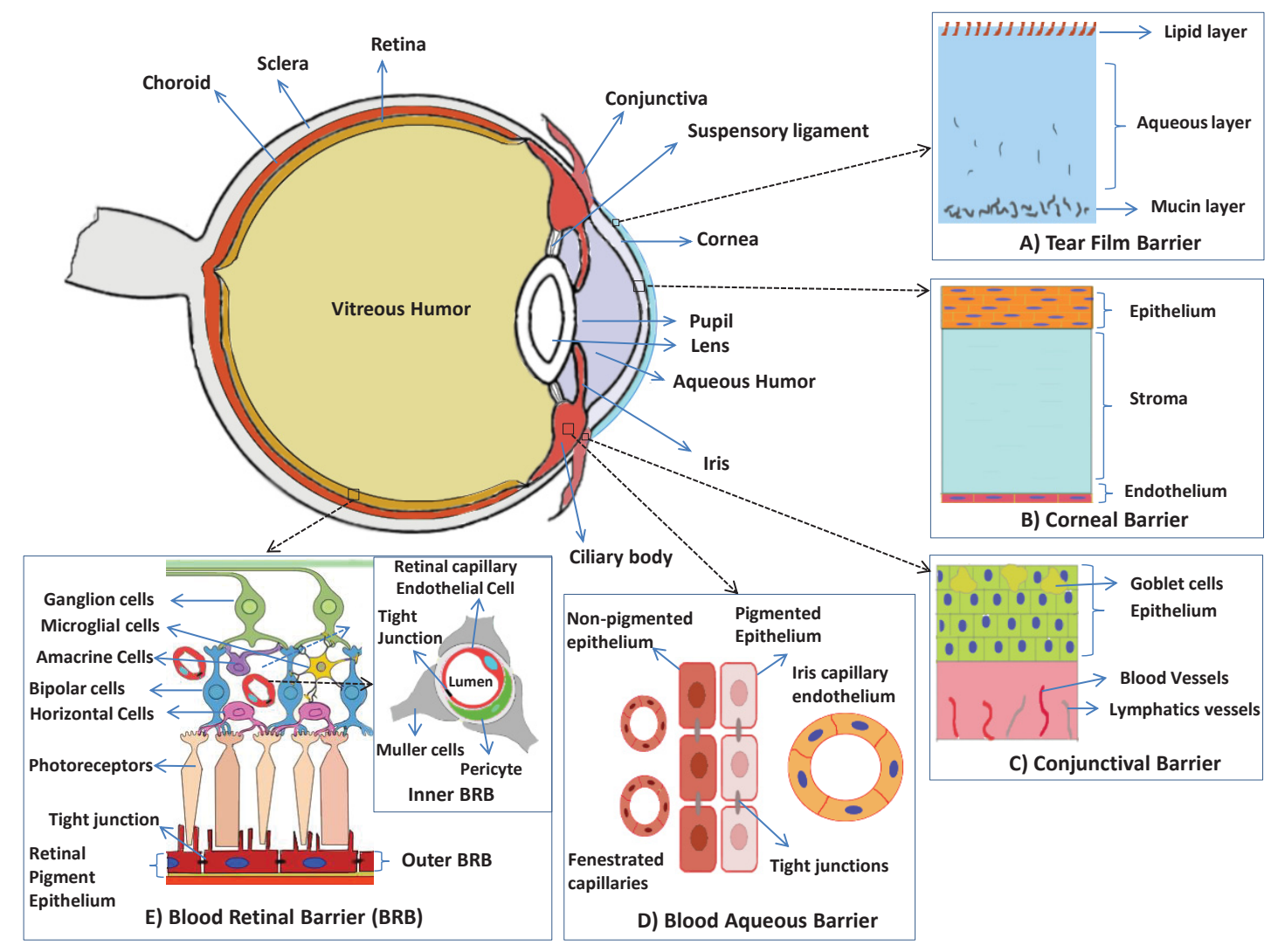

Figure 3-1. The structure of the anterior segment of the eye and ocular barriers for drug delivery.

Notes: A) Tear film barrier: Main components of the tear film include mucins, water, and lipid, and acts a defensive barrier to the foreign-object access to the cornea and conjunctiva. B) Corneal barrier: avascular and comprised of three major layers which are epithelium (multiple layers stacked on each other), stroma and endothelium (single layer). Acts as a barrier preventing the drug absorption from the lacrimal fluid into the anterior chamber after the topical administration. C) Conjunctival barrier: mucous membrane consisting of conjunctival epithelium (2-3 layers thick), and an underlying vascularized connective tissue. Acts a barrier to the topically administered drugs, and relatively inefficient compared to the corneal barrier. D) Blood-aqueous barrier: located in the anterior segment of the eye. Formed by the capillary endothelium in the iris, and the ciliary epithelium which both contain tight junctions. The barrier is relatively inefficient compared to the BRB, and small molecules can reach the aqueous humor by permeation through fenestrated capillaries in the ciliary processes. E) Blood-retinal barrier (BRB): located in the poster segment of the eye. Formed by the retinal pigment epithelium (outer BRB) and the endothelial membrane of the retinal blood vessels (inner $\mathrm{BRB}$ ), both contain tight junctions. The tight junctions restrict the entry of the drugs from the blood (systemic) into the retina/aqueous humor. 
Table 3-1. Administration routes for delivering drugs to the anterior segment of the eye.

\begin{tabular}{lll}
\hline Route of administration & Advantages & Limitation \\
\hline Topical & $\begin{array}{l}\text { simple, convenient, self-administrable } \\
\text { and noninvasive administration; } \\
\text { avoiding the blood-aqueous barrier; no } \\
\text { first-pass metabolism }\end{array}$ & $\begin{array}{l}\text { short contact time of drug on the } \\
\text { ocular surface; low efficiency and } \\
\text { low bioavailability due to corneal and } \\
\text { conjunctival barriers, tear clearance, } \\
\text { and nasolacrimal drainage }\end{array}$ \\
Intracameral & $\begin{array}{l}\text { avoiding the cornea, conjunctiva, and } \\
\text { blood-aqueous barrier; no first-pass } \\
\text { metabolism; high efficacy; high } \\
\text { bioavailability }\end{array}$ & $\begin{array}{l}\text { usually need reconstitution; correct } \\
\text { dosing and preparation are critical. }\end{array}$ \\
& $\begin{array}{l}\text { easy and minimally invasive } \\
\text { administration; avoiding the cornea and } \\
\text { blood-aqueous barrier; no first-pass } \\
\text { metabolism; good efficacy; good } \\
\text { bioavailability; sustained release }\end{array}$ & $\begin{array}{l}\text { conjunctival blood and lymphatic } \\
\text { clearance }\end{array}$ \\
& $\begin{array}{l}\text { direct delivery to the back of the eye; } \\
\text { surpass all the barrier; sustained release } \\
\text { (intravitreal implants) }\end{array}$ & $\begin{array}{l}\text { highly invasive; inherent potential } \\
\text { complications such as hemorrhage, } \\
\text { cataract, endophthalmitis, and retinal } \\
\text { detachment; rapid clearance }\end{array}$ \\
Intravitreal & $\begin{array}{l}\text { convenient to deliver a large dose of } \\
\text { the drug; noninvasive; avoiding the } \\
\text { cornea }\end{array}$ & $\begin{array}{l}\text { low bioavailability due to systemic } \\
\text { absorption and blood-aqueous } \\
\text { barrier; first-pass metabolism }\end{array}$ \\
\hline
\end{tabular}


limitations. In the following, we will discuss the details of the routes of administration and related ocular barriers.

\section{Topical Administration}

Topical administration is the simplest, convenient, self-administrable, noninvasive, and most dominant drug administration route for the management of eye diseases/disorders. It is a local drug delivery method, avoiding the blood-aqueous barrier, and the side effects and first-pass metabolism that may occur in some systemically administrated drugs. Drugs administrated through the topical route are usually formulated into eye drops. Depending on the formulation and drug's physiochemical characteristics, the drugs can reach various external (cornea, conjunctiva sclera) and internal (iris, ciliary body, aqueous humor, vitreous humor, retina) sites in the eye after topical instillation [250]. However, only $1-7 \%$ of the administered drugs can reach the intraocular tissues due to the tear film, cornea and conjunctiva barriers and another obstacle such as lachrymal drainage, tear drainage and tear turnover [246, 251, 252]. Tear film is the first obstacle faced for topically administered drugs. It consists of three layers: an outermost lipid layer, a thicker aqueous middle layer and an innermost mucin layer (Figure 3-1A). Due to the fast turnover rate and time of tear film, the topically administered eye drops are quickly washed away and drained into nasolacrimal duct after instillation [253]. Cornea is the second ocular barrier limiting the penetration of exogenous substances into the eye. It is composed of five layers: epithelium, Bowman's membrane, stroma, Descemet's membrane, and endothelium (Figure 3-1B). The layers which form substantial barriers to drug penetration are: epithelium, stroma and endothelium. The superficial corneal epithelium is composed of multiple layers of stratified squamous nonkeratinized epithelial cells. It limits the permeation of hydrophilic drugs through the cornea due to the hydrophobicity of the epithelium and the presence of tight junctional proteins between the corneal epithelial cells [250, 254]. The inner layer next to the corneal epithelium is stroma. The stroma is comprised of glycosaminoglycans and collagen fibrils in lamellar structures, and has hydrophilic environment. It restricts the penetration of lipophilic drugs through the cornea [250]. The innermost layer of the cornea is monolayer corneal endothelium composed of hexagonal-shaped endothelial cells. This corneal endothelium is leakier than the epithelium and allows the penetration of macromolecules into the aqueous humor on the other side [250]. Overall, cornea acts as a physical barrier to hydrophilic drugs due to the superficial corneal epithelial layers; and to lipophilic drugs due to the stroma. Besides the cornea route, topically administered drugs can be absorbed into the eye through non-cornea route: the conjunctiva/sclera pathway as the conjunctiva has a large surface area than the cornea $(17 \mathrm{vs} . \sim 1 \mathrm{~cm} 2)$ [251, $252,255,256]$. Conjunctiva is a rate-limiting barrier for permeation of water-soluble drugs $[257,258]$ due to rapid drug elimination by conjunctival blood and lymphatic flow (Figure 3-1C). After escaping from the conjunctival elimination, drugs penetrate through the sclera to inner ocular tissues (trans-scleral pathway). 


\section{Intracameral Administration}

Intracameral administration is a method for direct injection of drugs into the anterior chamber in the anterior segment of the eye [259]. It is a local drug delivery method, avoiding the side effects and the first-pass metabolism that may occur in some systemically administrated drugs. It also avoids the cornea, conjunctiva and bloodaqueous barriers. Therefore, intracameral injection can deliver drugs to the eye relatively easily with high efficiency and was expected to achieve 300 to 600 times aqueous humor drug level than topical application [260, 261]. After intracameral injection, the mechanism for drugs to reach the inner ocular tissues was via diffusion and the bulk flow of aqueous humor. However, intracameral antibiotics usually need reconstitution including dilution and other special preparations which require sterilization, nonpreservatives, and proper concentration and dose. If incorrect dosing and preparation occur, corneal endothelial toxicity and toxic anterior segment syndrome become significant concerns [261,262].

\section{Subconjunctival Administration}

Subconjunctival administration (periocular routes) places drugs in the subconjunctival space around the outside of the sclera, and then drugs penetrate through the sclera into the eye. This route is safer and less invasive and effective for delivering drugs to the eye, avoiding the cornea and blood-aqueous barriers, and the side effects and the first-pass metabolism that may occur in some systemically administrated drugs. It is a local drug delivery method, and can offer sustained drug delivery depending on formulations or devices. Through this route, the primary pathway to reach the retina is via sclera and underlying choroid-Bruch's layer and retinal-pigment epithelium (RPE). The sclera is the white outer elastic and microporous tissue layer comprised of proteoglycans and closely bundled collagen fibers, containing about $70 \%$ water. The pathway for drugs to diffuse across sclera is through the interfibrillar fluid media of the gellike proteoglycans. Sclera has large surface area and relatively high permeability than cornea, and the transscleral permeation mainly depends on the size of the drug molecules rather than their lipophilicity $[250,263,264]$. However, the subconjunctival route has limitation of possible loss of drugs to the systemic circulation due to the drainage via the conjunctival blood and lymphatic vessels [263, 265].

\section{Intravitreal Administration}

Intravitreal administration is a highly invasive route that provides the direct approach for delivering medications to the back of the eye with a greater probability of achieving effective drug concentrations in the posterior tissues while lowering the risk of systemic exposure. Several of the current therapeutics (eg. Avastin, Lucentis, Dexamethasone) relies on intravitreal administration for treating posterior eye diseases. Although intravitreal route is very advantageous as it overcomes all the ocular barriers and allows direct entry of drugs into the back of the eye, it suffers from various inherent potential complications such as hemorrhage, cataract, endophthalmitis, and retinal detachment. Because of its invasive nature, it lacks the patient compliance. Moreover, 
drugs administered directly into the vitreous humor undergo rapid elimination. To avoid multiple injections, intravitreal sustained release systems such as implants, microspheres, liposome can be used. However, intravitreal implants too suffer from similar complications as intravitreal injections.

\section{Systemic Administration}

Systemic administration can deliver drugs to the anterior segment and posterior segment of the eye, but with low bioavailability due to the presence of the BAB, and $\mathrm{BRB}$. The two layers that comprise the BAB are the endothelium of the iris/ciliary blood vessels and the nonpigmented epithelium of the ciliary body (Figure 3-1D) [266]. BRB is located in the poster segment of the eye. As the retina is in contact with the highly vascularized chordal tissue which has fenestrated capillaries, there are two BRBs. It is formed by the retinal pigment epithelium (outer BRB) and the endothelial membrane of the retinal blood vessels (inner BRB), both contain tight junctions (Figure 3-1E). The outer BRB is a monolayer of tightly joined RPE and separates the posterior side of the retina from the choroid. Both outer and inner BRBs have tight junctional complexes and limit the penetration of blood-borne components into the aqueous humor/retina. However, both the layers are expressed by wide range of selective transporters for the solutes. In order to achieve therapeutic levels of drugs in the internal ocular tissues, high doses are necessary. High doses can cause adverse systemic side effects so that systemic administration route is rarely used to treat eye disease/disorders.

\section{Blood Brain Barrier*}

$\mathrm{BBB}$ is the protective interface that separates the brain from the systemic circulation and this barrier has several common features as outer BRB (the retinal endothelium, Figure 3-1E). Anatomically, the BBB is composed of endothelial cells which are lining the cerebral microvessels and buttressed by astrocyte and pericyte cells (Figure 3-2) [267, 268]. The BBB functions as a physical barrier due to tight junctions between the adjacent endothelial cells, a selective 'transport barrier' due to specific transport systems on the luminal and abluminal membranes of the endothelial cells, and a 'metabolic barrier' due to the presence of intracellular and extracellular enzymes [269272]. The tight junctions are composed of three integral membrane proteins including claudin, occludin, and junction adhesion molecules, and a number of cytoplasmic accessory proteins including ZO-1, ZO-2, ZO-3, cingulin, etc. [267, 269]. Fully developed tight junctions serve as a gatekeeper to restrict the paracellular transport across the BBB. The transport systems are influx and efflux transporters. The influx transporters

\footnotetext{
* Adapted with permission from "Pharmaceutical research. Overcoming the blood-brain barrier in chemotherapy treatment of pediatric brain tumors. 31(3). 2013 . p. 531-540. Wu, L., Li, X., Janagam, D. R., Lowe, T.L. (C) Springer Science + Business Media New York 2013. With permission of Springer."
} 




Figure 3-2. Anatomy of the blood-brain barrier (BBB). 
include solute carrier superfamily facilitating brain uptake of glucose, amino acids, ions, and other nutrients; while the efflux transporters are ATP-binding cassette (ABC) efflux transporters such as P-glycoproteins and multidrug resistance-associated proteins [273], which efflux lipophilic toxins including many therapeutics away from the brain to reduce the penetration of these molecules into the brain. The intracellular and extracellular enzymes are monoamine oxidase and cytochrome P450 (CYP1B1), and ectoenzymes, respectively, responsible for inactivating many neuroactive and toxic compounds in the BBB [267, 272, 274]. While the three physical, transport and metabolic barriers allow nutrients in the blood stream and waste generated in the brain to cross the intact BBB, they prevent or limit non-selected substances to penetrate the intact BBB and get into the brain. It has been well-known that $98 \%$ of small molecule drugs with a molecular weight smaller than $400 \mathrm{Da}$ including many chemotherapeutics such as carboplatin, vincristine, cyclophosphamide, cisplatin, methotrexate and etoposide cannot cross the intact BBB to get into the brain. Almost $100 \%$ of large molecule drugs with a molecular weight greater than $400 \mathrm{Da}$ also cannot penetrate the intact BBB by themselves [270].

\section{Strategies for Circumventing the BBB}

The strategies to circumvent/overcome the limitation of the BBB could be categorized into i) by-passing the BBB by directly administrating therapeutics into the brain, ii) disrupting the $\mathrm{BBB}$ by temporarily opening the tight junctions in the $\mathrm{BBB}$, iii) active transport across the $\mathrm{BBB}$ by exploiting transport mechanisms associated with the $\mathrm{BBB}$, and iv) by-passing the $\mathrm{BBB}$ by directly delivering therapeutics to the brain through intranasal nose to brain route [38, 50, 275-277].

\section{By-passing the BBB}

Drugs could be directly administrated into the central nerve system (i.e. brain and spine), thus bypassing the BBB and blood-cerebrospinal fluid (CSF) which are formidable obstacles faced by systemic administrated (e.g. oral and intravenous) drugs. Intrathecal administration and convection-enhanced delivery are mainstays of such administration methods (Figure 3-3). For intrathecal administration, drugs are injected into the fluid-filled space in spine via a needle or under the scalp via an outlet catheter connected to the ventricles (Figure 3-3A) [278-280]. Compared to systemic administration in which the whole body acts as a sink for the administrated drugs, the intrathecal administration needs much lower drug dose to achieve higher drug concentrations in the brain due to the small volume of CSF (about $150 \mathrm{ml}$ ). Moreover, as the drugs are administrated behind the BBB and thus the BBB is not a concern or obstacle for administration through this method. Intrathecal drug administration has been used in the management of brain diseases such as brain tumors for several decades [279, 281-285]. One disadvantage for intrathecal administration is that intrathecal drug administration can result in non-targeted drug distribution, inhomogeneous dispersion, and ineffective volume of drug distribution due to drug molecular weights and infuscate diffusivities. 


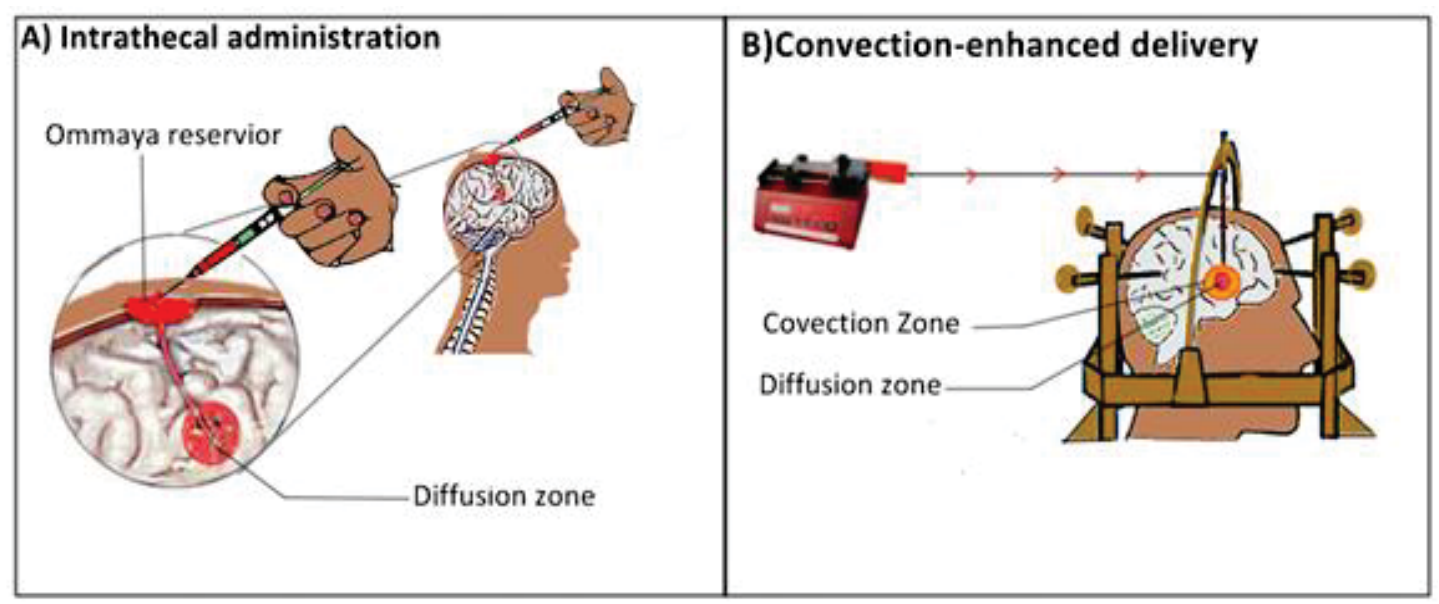

Figure 3-3. Intrathecal administration (A); Convection-enhanced delivery (B).

Notes: Intrathecal administration and convection-enhanced delivery are the methods that directly administer the drugs into the central nerve system (i.e. brain and spine), thus bypassing the BBB and blood-cerebrospinal fluid (CSF) [280, 286, 287]. 


\section{Convection-enhanced Delivery (CED)}

CED is another regional drug administration method introduced by Oldfield and his associates to overcome the limitations for intrathecal administration [286]. In CED, intracranial catheters are connected to target sites to deliver therapeutics under a continuous pressure gradient over periods of hours to days and thus enhance the distribution of drugs by convection rather than diffusion (Figure 3-3B) [288]. This method allows delivery of high concentration of drugs directly into diseased sites in the brain and the adjacent parenchyma, thus eluding the BBB and limiting systemic toxicity. Small molecule drugs, macromolecules, and even nanocarriers have been successfully method allows delivery of high concentration of drugs directly into diseased sites in the brain and the adjacent parenchyma, thus eluding the BBB and limiting systemic toxicity. Small molecule drugs, macromolecules, and even nanocarriers have been successfully delivered into the brain using CED [286-290]. CED has been employed in the clinic and clinical trials to manage brain diseases [288-293]. For instance, in the case of treating cancer, positive response and tumor regression have been observed in the clinical studies using CED to administer drugs [293, 294]. Even though CED, and intrathecal injection are effective in delivering drugs into the brain, these two administration methods are invasive in general.

\section{Disrupting the BBB}

The BBB can be disrupted by osmotic means, vasoactive substances, and focused ultrasound [295-304]. The disruption induced transiently opens the BBB and thus allows therapeutics, which are generally prevented from entering the brain, to pass this barrier and get into the brain tissues. In the osmotic BBB disruption approach, a hypertonic solution is infused into the arterial blood to cause brain microvascular endothelial cells in the BBB transiently and reversibly shrink so that the tight junctions in the BBB is transiently opened to allow the entrance of hydrophilic therapeutics into the brain [295]. Mannitol is the most widely used hyperosmotic agent for this osmotic BBB disruption. In the clinic, osmotic BBB disruption has been used to deliver chemotherapeutics into the brain for decades [298, 305-309]. However, this osmotic BBB disruption is global, nonselective disruption covering both diseased areas and normal brains region. This nonselective opening of the BBB may allow the entrance of other substances which might lead to adverse effects as seizures and chronic neurological changes [310]. A delayed recovery of the $\mathrm{BBB}$ also increases the risk of neurotoxicity [310]. These stimulated researchers and clinicians to seek methods to open the BBB at the diseased lesions selectively. Vasoactive substances were found to be capable of stimulating receptors preferentially expressed in the brain tumor vascular vessels over normal brain vessels and thus initiate second messenger systems that induce reversible opening of the tight junctions in the brain tumor vascular vessels [310]. This unique property was exploited by researchers and clinicians to open the BBB at the diseased sites selectively [297, 310-313]. 


\section{Active Transport across the BBB}

It has recently been recognized that therapeutics could be successfully delivered into the brain after intravenous injection by crossing the BBB without the need of disrupting the $\mathrm{BBB}$. The methods that have been developed are to utilize the transport mechanisms found in the BBB to rationally design drugs and/or drug delivery systems to enhance drug permeability across the BBB. The transport mechanisms exploited include the transcellular lipophilic pathway responsible for the uptake of some lipid-soluble agents, carrier-mediated transcytosis for the transport of glucose, amino acids, purine bases, nucleosides, choline and other substances, receptor-mediated transcytosis for certain proteins such as insulin and transferrin, and adsorptive-mediated transcytosis for native plasma proteins such as albumin [267]. Especially, in the recent 15 years nanocarriers such as polymeric micelles [314], dendrimers [315], polymer-drug conjugates [316, 317], polymeric nanoparticles [318], liposomes [319-321], and inorganic nanoparticles including gold [322], mesoporous silica [323-325] and superparamagnetic iron oxide (SPIO) [326, 327] were investigated. In some cases, cell penetrating peptides such as TAT (trans-activator of transcription) or receptor targeting molecules such as transferrin, OX26 (anti-transferrin receptor IgG2a antibody) have also been conjugated to the nanocarriers to enhance drug penetration through the $\mathrm{BBB}$ after intravenous injection as transferrin receptor is expressed on the BBB [328-330].

\section{Intranasal Nose to Brain Delivery}

In the recent years, several reports have been published on the intranasal nose to brain delivery where the olfactory region of the nasal mucosa located on the roof of the nasal cavity that provides a direct connection between nose and brain was exploited for delivering drugs to the brain [331-333]. Advantages of this route include noninvasiveness, circumvention of the BBB, escape hepatic first-pass metabolism, simple, and convenience of administration. Therapeutics have been shown to reach the central nervous system through the olfactory neuroepithelium located at the loft of the nasal cavity, by trigeminal nerve systems and olfactory nerve pathways [331-333].

\section{Nanoparticle-based Drug Delivery}

In an attempt to surpass the limitations associated with the aforementioned barriers, considerable investigation has been done for improving the efficacy of drugs in vivo and such attempts resulted in the design, study and use of nanoparticulate drug delivery systems, such as biodegradable polymeric nanoparticles [334], nanocapsules [335], liposomes [336], dendrimers [62-66], micelles [314], inorganic nanoparticles [78]. Various nanoparticles with their structures are illustrated in Figure 3-4. Currently, one of the most interesting fields of research in drug delivery is the design of nanoparticles capable of delivering the therapeutics to the right site predictably over time $[38,49,50$, 275-277, 315, 317, 321, 337-342]. These are submicron sized systems (1-1000 nm) containing the encapsulated drugs intended for administration [18]. Because 


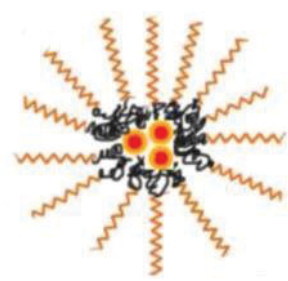

a. Polymeric Micelle

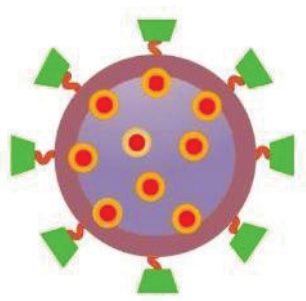

d. Polymeric Nanoparticle

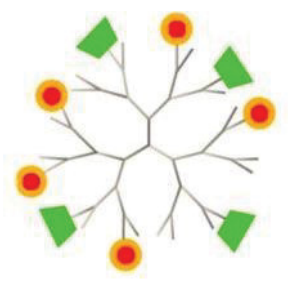

b. Dendrimer

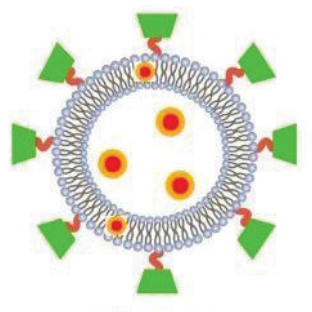

e. Liposome

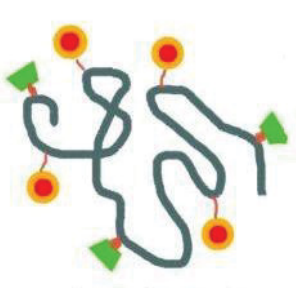

c. Polymer-drug Conjugate



f. Inorganic Nanoparticle

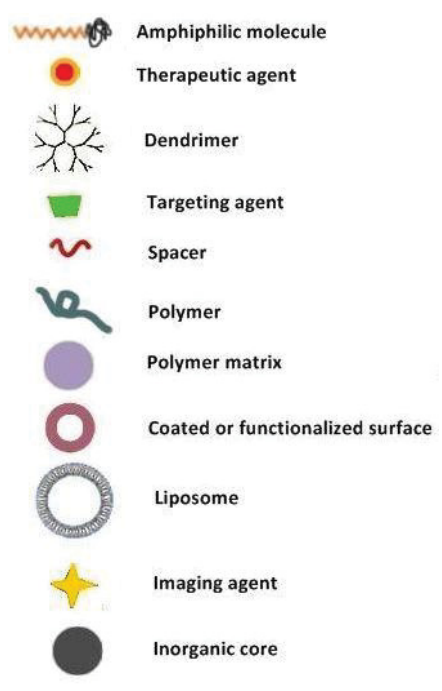

Figure 3-4. Nanocarriers for brain drug delivery.

Notes: Nanocarriers for brain delivery [343]: a. Polymeric micelles: core-shell nanosized structures formed by a spontaneous self-assembly of polymers as a result of ionic or hydrophobic interactions between polymer chain segments [314]; b. Dendrimers: macromolecules with highly branched 3D structure offering a high degree of surface functionality and versatility [315]; c. Polymer-Drug conjugates: nano-sized and multicomponent constructs with therapeutics and targeting ligands covalently attached to the polymer chains [316, 317]; d. Polymeric nanoparticles: submicron sized particles prepared from pre-synthesized polymers or through in-situ polymerization from monomers/macromers directly [318]; e. liposomes: spherical and self-closed lipoidal vesicles (unilamellar or multilamellar) of colloidal dimensions formed as result of selfassembly of phospholipids in an aqueous media into closed bilayered structures [319321]; and f. inorganic nanoparticles: nanosized inorganic core including gold [322], mesoporous silica [323-325] and SPIO [326, 327], coated with polymers and conjugated with targeting and imaging agents on the surface $[344,345]$. Reprinted with permission. Wu, L., et al., Overcoming the blood-brain barrier in chemotherapy treatment of pediatric brain tumors. Pharmaceutical research, 2014. 31(3): p. 531-540. 
nanoparticles are small-sized with large surface area and can be made of many types of safe materials (such as synthetic biodegradable polymers, natural biopolymers, lipids) with multi-functional surface groups, they possess outstanding advantages such as: i) can pass through the smallest capillary vessels; ii) potential of cellular internalization of drug; iii) hold significant advantages in transporting drugs across biological barriers; iv) reducing drug clearance in the path by protecting drugs from degradation and metabolism, and improving drug stability and bioavailability at the target site; v) sustained drug release which consequently leads to reduction in dosage frequency, resulting in better patient compliance; vi) reduced incidence of toxicity and side effects,;vii) maintaining long shelf-life; viii) biocompatible and biodegradable. [18, 37, 81-86].

Among various nanoparticles, polymeric nanoparticles are gaining increasing attention and have been extensively studied for imaging, diagnosis and drug delivery due to their superior properties over others [37-43]. Therapeutics are loaded into the nanoparticles by adsorption, entrapment, and covalent attachment. They are released from the nanoparticles by desorption, diffusion, drug dissolution and polymer erosion, or some combination of these mechanisms. They can be optimized to have higher permeability across the specific barriers and/or prolonged contact time with the tissue surfaces by adjusting their properties such as particle size, surface charge, and surface chemistry [346, 347]. Meanwhile, targeted delivery can also be achieved by conjugating specific targeting moieties to the nanoparticles [67, 348-351]. Since the polymeric nanoparticles can be made of various types of materials, have large surface areas, and multi-functional surface groups these systems hold a great promise and were widely explored for transporting therapeutic agents across various biological barriers such as the ocular/blood-brain barriers. Various biodegradable polymers that have been used for the preparation of these nanoparticles are polysaccharides (chitosan, dextran, and hyaluronic acid), polyesters (PLA, PLGA, PCL), PVA, polyacrylic acid family, proteins or polypeptides (such as gelatin), etc. Among them, polysaccharides are the most popular biopolymers used for the fabrication of the nanoparticles for drug delivery because of their wide availability in nature, low-cost processing, biocompatibility, biodegradability, non-toxicity, hydrophilicity and bioactivity $[18,352]$.

\section{Polysaccharide-based Nanoparticles for Drug Delivery to the Eye and Brain}

Polysaccharides are long chain carbohydrate molecules where the monosaccharide units are linked through a glycosidic linkage which undergoes enzymatic degradation. Most common sources of polysaccharides are plant (starch, cellulose, carboxyl methyl tamarind, pectin), animal (chitosan, chitin, hyaluronic acid), microbial (dextran, pullan, gellan gum, xanthan gum), algae (alginate, agar). Table 3-2 lists out some of the commonly used poly-, oligosaccharide polymers, and their structures. They have a large number of reactive functional groups varying in a chemical composition and having a broad range of molecular weight thus contributing to their diversity in structures and properties. Common hydrophilic groups present in the polysaccharides are hydroxyl, carboxyl, and amino groups. These functional groups in polysaccharides can form 
Table 3-2. Chemical structures of various polysaccharides that were used for drug delivery.



Sodium alginate

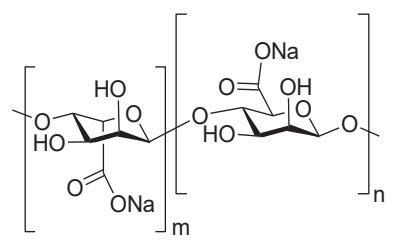

Carboxymethyl tamarind kernel polysaccharide

Pectin
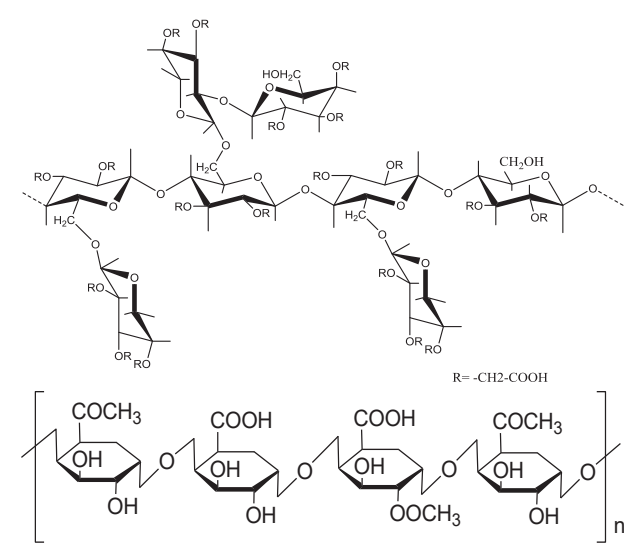
Table 3-2. (Continued.)

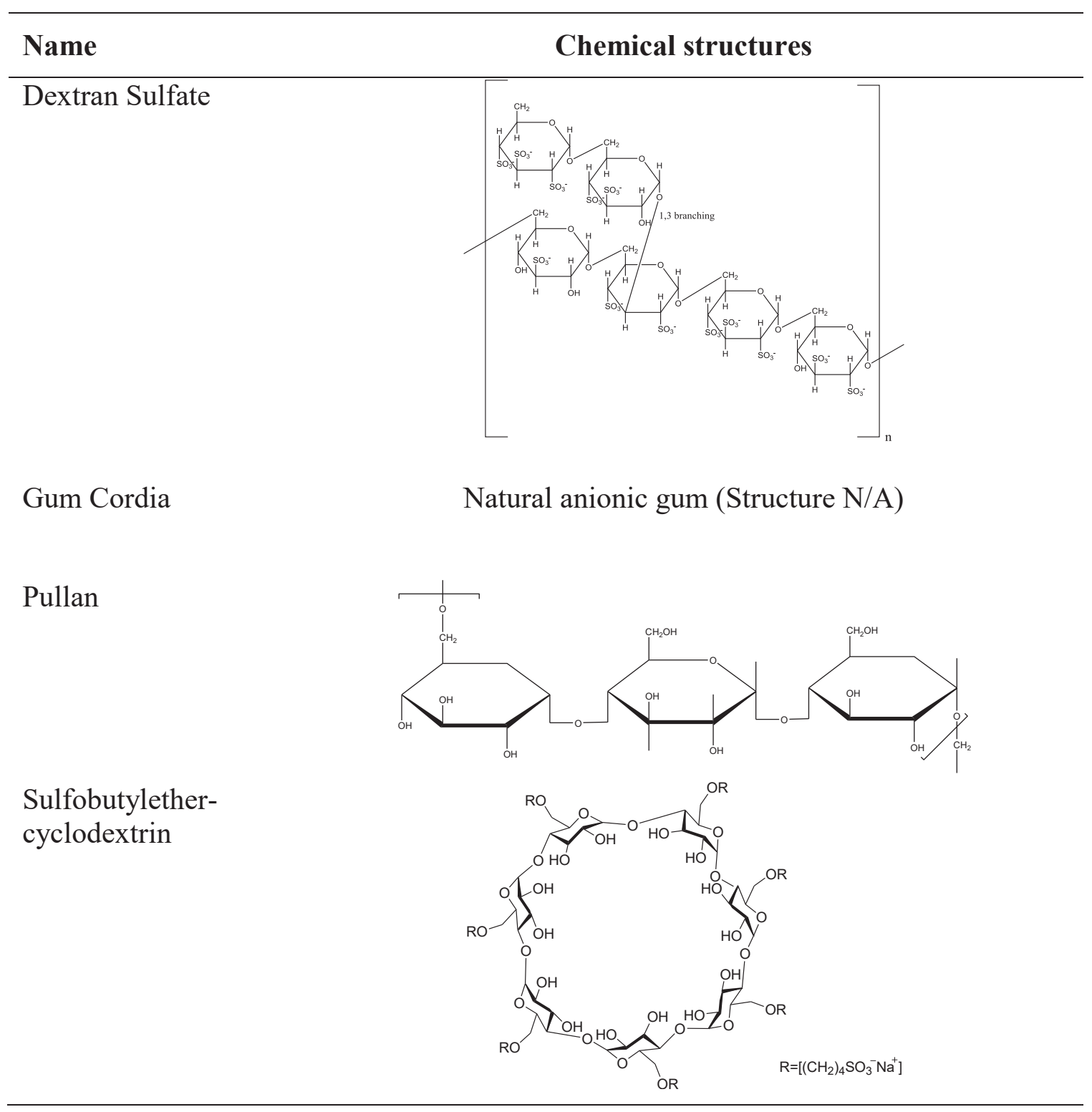


hydrogen bonds with mucosa. Thus, their mucoadhesive nature depends on the noncovalent bond forming ability [353]. These bioadhesive polymers are used as carriers as they could prolong the residence time of the drugs. Besides the mucoadhesive property, nature-originated polysaccharides such as chitosan and its derivatives, dextran, alginate, hyaluronic acid, gum cordia, and carboxymethyl tamarind kernel polysaccharide, cyclodextrins (Table 3-2) possess other appealing properties such as acceptable biocompatibility, excellent in vivo tolerance [354-356], biodegradability [357], and ability to enhance drug membrane permeability both in vitro [358] and in vivo [359]. Certain polysaccharides possess some special characteristic capability to recognize special cell types, enabling to design targeted drug delivery systems through receptormediated endocytosis, while others can be effectively modified with different polymers and utilized as a part of the drug delivery systems. The combination of these properties makes polysaccharides versatile biopolymers for drug delivery to the eye and brain [18, $67,68,349,360-373]$. Also, availability of new synthetic routes can be adopted to easily modify polysaccharides or synthesize oligosaccharide units, tunable biodegradability by structural modification, and ability to fabricate suitable structures such as nanoparticles, make them one of the most important and extensively studied natural biomaterials for nanoparticle-based drug delivery applications [352, 374-376]. Initial studies were mainly focused on evaluating chitosan-based nanoparticle carriers, and in the long run more number of polysaccharides (dextran, pectin, gum cordia, carboxymethyl tamarind kernel polysaccharide, hyaluronic acid, mannan, maltodextrins, and pullan) have emerged for the formulation of nanoparticles thereby increasing the versatility of nanoparticle carriers in terms of category and function [18, 346, 377-383]. A brief discussion is given below about the polysaccharides of interest to this project which includes Dextran and Cyclodextrins.

Dextran is one of the widely investigated natural polysaccharide that has been successfully used for drug delivery applications due to its excellent biocompatibility, biodegradability, hydrophilicity, and nonfouling properties [384-388]. Dextran is enzymatically degradable by dextranases present in various parts of the human body. For more than five decades dextran has proven its clinical safety as antithrombotic agents and as substitutes for plasma volume expansion [385]. It is produced industrially on a large scale, and there are many varieties of dextran polymers that are commercially available in wide range of MWs and with relatively narrow MW distribution. The degree of branching in this polysaccharide can be as small as $0.5 \%$, making them suitable for scientific studies [385]. Apart from being water soluble, dextran polymers are also stable under slightly acidic and basic conditions, and also possess a large number of hydroxyl functional groups for conjugation [385]. Recently, several reports have been published on altering the dextran properties to make various detrain graft polymers via various chemical modifications for specific applications, for example, polymeric drug delivery systems and hydrogels [388-391]. Although dextran has been widely investigated for various drug delivery applications, only limited number of papers have been published on dextran-based nanoparticles for drug delivery to the eye or brain [372, 392-396]. Chaiyasan et al. synthesized hybrid nanoparticles made of chitosan and dextran sulfate with around $400 \mathrm{~nm}$ particle size and around $40 \mathrm{mV}$ zeta potential, which were stable in the presence of lysozyme for at least $4 \mathrm{~h}$ and showed at least 60 min prolonged adherence 
to the ex vivo porcine corneal surface [372]. Phenylboronic acid surface functionalized poly(D,L-lactide)-b-dextran nanoparticles were investigated by Liu et al. for ocular delivery, and the results demonstrated that nanoparticles were nontoxic and can sustain the drug (cyclosporine A) release for 5 days in vitro [397]. Dextran polymer was also used as a stabilizer during the nanoparticle preparation [398]. Alonso et al. found that PBCA nanoparticles made with dextran $70 \mathrm{kDa}$ as the stabilizer has shown statically significantly increased drug concentrations in the cornea and aqueous humor compared to the other nanoparticle formulations made with other stabilizers (Synperonic F 68 and sodium lauryl sulfate) and the control amikacin sulfate drug solution [334]. Delgado et al. studied dextran and protamine-based solid lipid nanoparticles as potential non-viral vectors for ocular gene delivery and found that the vectors were able to transfect corneal cells after topical application. Here dextran was used to form polyplex with the protamine and then combined with SLN to obtain dextran-protamine-DNA-SLN hybrid non-viral vectors [393]. Another group studied the hybrid nanoparticle design based on cationized gelatin and the polyanions dextran sulfate for ocular gene delivery, and the results showed that nanoparticles were nontoxic to the corneal cells and were able to protect the DNA from degradation by DNase I [394]. Dextran was also successfully used in making nanoparticles with magnetite for imaging the BBB breakdown after experimental hyperosmotic disruption [392]. Further, following this BBB disruption, when the magnetite-dextran nanoparticles were injected into the carotid artery, they have penetrated into the brain tumor [396]. Although dextran has been studied for making nanoparticles for ocular delivery, to the best of author's knowledge no report has been published on dextran-based nanoparticles that can cross the ocular barriers and sustain the drug release at the back of the eye. Therefore, in this project, the dextran polysaccharide was modified to improve the properties and practicability of polymeric nanoparticles for ocular drug delivery applications with the potential to overcome the ocular barriers. The modifications or the procedure include i) hydrolytically degradable component was incorporated into the dextran polymer chain by grafting with PLA units; ii) polylactide grafted dextran was then copolymerized with NIPAAm and acrylic acid (AA) using emulsion photopolymerization to from poly(NIPAAM-co-DEXlactateHEMA) nanogels. The addition of NIPAAm and AA imparts thermoresponsive and anionic nature (carboxylic groups) to the nanogels. Because of the amphiphilic nature of the dextranlactide, the nanogels were synthesized in an entirely aqueous solution without the need of surfactants and organic solvent. The size, charge, and degradability of this dextran-based nanogels can be easily tuned by altering the composition.

Another polysaccharide-based material used in this work was derived from $\beta$-CD. Cyclodextrins are cyclic oligosaccharides consisting of $6(\alpha-C D), 7(\beta-C D)$ or $8(\gamma-C D)$ glucopyranose units, which form a hydrophobic inner cavity and hydrophilic outer surface. The size of this inner hydrophobic cavity is the significant determinant as to which cyclodextrin is utilized as a part of complexation. The interior cavity size of $\beta-C D$ was found to be the most appropriate size suitable to accommodate many of the drugs. $\beta$ $\mathrm{CD}$ and its derivatives have a unique property of forming inclusion complexes with hydrophobic drugs and increase the drugs' water solubility [399, 400]. The $\beta$-CDs have been extensively used in eye drops for improving the solubility of the drugs, and improve their bioavailability. $\beta-\mathrm{CD}$ also offer a number of hydroxyl surface functional groups for 
further conjugation or alteration via various chemical modifications for specific applications. $\beta-C D$ and its derivatives have been widely used in various drug formulations such as eye drops, nasal spray, and oral and parenteral preparations to increase the solubility and stability of hydrophobic drug molecules and reduce their irritancy/toxicity. Additionally, several studies have reported the use of synthetic $\beta$-CDbased polymers units for the drug delivery applications [87, 370, 401, 402]. Although drug/cyclodextrin complexes have been widely employed in the past, incorporating them into nanoparticles seems to bring significant advantages. In ocular application, sulfobutylether- $\beta$-cyclodextrin was used to make hybrid nanoparticles with chitosan to form inclusion complexes with econazole nitrate to increase the water solubility of econazole nitrate. The resulting econazole nitrate-containing complexes showed improved bioavailability and prolonged 8 hours' steady antifungal effect while econazole nitrate solution could provide antifungal effect for only 3 hours which also dramatically decreased with time [370]. Tao et. al. demonstrated that hydroxypropyl- $\beta$-CD during the fabrication of puerarin-PLGA nanoparticles has improved the solubility and entrapment efficiency of the puerarin, and also proposed that nano-carriers or cyclodextrin inclusion complex enhanced the penetration of drug across the BBB [403]. Gill et. al from our group has earlier modified $\beta-\mathrm{CD}$ to generate cationic quaternary ammonium - $\beta-\mathrm{CD}(\mathrm{QA}-$ $\beta$-CD) nanoparticles for potential drug delivery across BBB [87]. When doxorubicin was loaded into these nanoparticles, they enhanced the permeability of doxorubicin across the in vitro $\mathrm{BBB}$ model and masked its cytotoxicity to the endothelial cells of the BBB [87]. As biodegradable polymers can achieve sustained drug release and polyamine can enhance drug permeability across the BBB, another $\beta$-CD-based poly( $\beta$-amino ester) (CD-p-AE) nanoparticles were designed, which contains ester bond and amine, into $\beta$-CD [50]. These nanoparticles also showed sustained release of doxorubicin and showed enhanced permeability across in vitro BRB models. The $\beta$-CD based nanoparticles have demonstrated promising potential as drug carriers for brain delivery [50]. In this study, (CD-p-AE) nanoparticles was selected for conducting the in vivo studies to evaluate the potential for delivering $17-\mathrm{AAG}$ to the brain.

\section{Diabetic Retinopathy}

Diabetic retinopathy (DR) is a chronic eye disease and one of the most frequent complications of diabetes. DR is affecting $60-75 \%$ of persons with diabetes and is the leading cause of visual impairment in working-age Americans [404]. Retinal neuronal damage and increased vascular permeability occurs in early phases of diabetes and may lead to clinically noticeable vascular changes [404]. Insulin has been investigated as a potential therapeutic option to protect the retinal neurons from apoptosis and prevent the neuronal degeneration [405-407]. Studies showed that intensive systemic insulin therapy lowers the risk of retinal neural apoptosis in diabetic rats, and systemic glycemic control with systemic insulin treatment lowers the risk of the development and progression of DR in diabetic human subjects (Diabetes Control and Complications Trial, 1994). However, the degree of systemic insulin treatment is restricted by the risks of hypoglycemia, including seizures and unconsciousness [4]. Therefore, there is a need for long-term local delivery of insulin to the retina. Our group has attempted to address this need by 
developing insulin-loaded biodegradable chemically cross-linked poly(NIPAAm-coDEXlactateHEMA) hydrogels for local delivery of insulin in the eye after subconjunctival administration [408]. Previous work from our group demonstrated that Humalog ${ }^{\circledR}$ (insulin lispro) could be continuously released from subconjunctivally implanted hydrogels for one week and showed a reduction of DNA fragmentation in the retinas of diabetic rats [409]. Even though the hydrogels can continuously release insulin at the subconjunctival space, insulin still needs to cross the sclera and the BRB and to access the retina by itself and faces the issue of low bioavailability in the retina. Therefore, there is a need for an effective drug carrier that can enhance the permeability of insulin across various ocular barriers. Nanoparticles show great promise for transporting drugs across biological barriers, reducing drug clearance, and improving the bioavailability of drugs at targets [36]. However, no nanoparticle has yet been developed to deliver drugs across the BRB effectively.

\section{Alzheimer's Disease}

Alzheimer's disease (AD) is the most common form of dementia characterized by progressive memory loss and cognitive dysfunction [410]. According to Alzheimer's Association report 2016, more than 5 million people are living with AD in the United States, and a new case is expected to develop every 66 seconds. AD is the 6th leading cause of death in Americans [411]. The disease development mechanisms of AD are still not entirely clear [412]. However, AD is characterized by the potentially neurotoxic aggregation of $\beta$-amyloid $(A \beta)$ peptide and tau protein within the brain, and their deposition as amyloid plaques and neurofibrillary tangles (NFTs) [413, 414]. Common therapeutic approaches in case of AD treatment include lowering the brain concentrations of $A \beta$ and tau or inhibiting their aggregation [414]. The selective neuronal loss observed in $\mathrm{AD}$, coupled with the growing body of evidence that chaperone protein heat shock protein 90 (Hsp90) inhibitors can clear protein inclusions (e.g. alfa-synuclein and tau) and rescue degenerating neuronal cells, has supported a therapeutic role for Hsp90 inhibitors in treating AD [415-418]. 17-Allylamino-17-demethoxygeldanamycin (17AAG), an Hsp90 inhibitor, have poor water solubility and short half-lives, do not or hardly cross the BBB, and can cause toxicity and side effects at the high dose. Though local invasive (direct injection/infusion) is effective in delivering drugs to the brain but has been associated with risk of infection, high neurosurgical cost and a limited delivery area in brain [45]. There is an unmet clinical need to develop effective methods to enhance the BBB permeability of 17-AAG and sustain the release of 17-AAG in the brain to treat $\mathrm{AD}$. According to a review of drugs for AD states that "successful treatment of $\mathrm{AD}$ will require drugs that can negotiate the BBB"[419].

\section{Objective}

Although there are several nanoparticles made of various materials such as polyesters (poly(D,L-glycolide-co-lactide) [44], poly(lactic acid) [45-47], poly( $\varepsilon$ -

caprolactone)[48, 49]); poly(amino ester)[50], lipid [liposomes [51-54], niosome [55, 56], 
solid lipid [57, 58]); polyamines [59-61], polyethyleneimine [420, 421]; polylysine [422] and dendrimers [62-66]; polysaccharide (chitosan [67-70] and hyaluronic acid [70]); polyalkylcyanacrylate [71-74]; albumin [75-77], and inorganic metals [78], however there are certain limitations associated with each of these nanoparticles. For instance, the polyester nanoparticles have shown to achieve sustained drug release, but not reported to cross the biological barriers themselves without the need to be conjugated or coated with some specific functional moieties [44-49]. Liposomes, solid lipid nanoparticles, dendrimers, surfactant-coated PBCA nanoparticles were reported to be able to cross the barrier; however, they cannot achieve sustained release and also can have toxicity issues $[57,73,79,80]$. The other nanoparticles have not been reported to be able to either sustain drug release or cross the barrier without the need to be conjugated or coated with some specific functional moieties. Therefore, there is still an unmet need to develop nanoparticles that can carry drugs across the ocular/blood-brain barriers and sustain the release of drugs to treat chronic ocular and brain diseases such as DR and AD. The primary objective of this work was to develop biodegradable polymeric biomaterials for drug delivery across ocular and blood-brain barriers for treating in the eye and brain, respectively. For this purpose two types of new nano-sized, cross-linked, hydrolytically degradable, and charged polysaccharide-based particles were prepared for drug delivery to the eye and brain which include a) poly(N-isopropyl acrylamide-co-dextran-lactate-2hydroyethyl methacrylate) [also referred to as Poly(NIPAAm-co-DEXlactateHEMA)] nanogels, and $\beta$-cyclodextrin-poly( $\beta$-amino ester) (CD-p-AE) nanoparticles. These materials combine the merits of biodegradable nature, charge, and nano-sized particulate properties. The degradation property of the polymeric matrix can be used to modulate the release of encapsulated drugs for an extended period of time and helps to avoid the removal of the system after treatment. Meanwhile, the surface charge and nano-sized dimensions impart the advantages of nanoparticulate systems such as internalization into the cells, transporting drugs across biological barriers, improving drug stability and bioavailability at the targets $[18,37,81-88]$. Additionally, the surface functional groups of these nanoparticles can be used for further conjugation with a ligand. Hence, in an attempt to minimize the constraints and disadvantages of conventional drug delivery systems for eye and brain treatment, a contemporary poly(NIPAAm-coDEXlactateHEMA)/ (CD-p-AE) nanoparticles based sustained drug delivery systems could be very promising.

\section{Biodegradable Poly(NIPAAm-co-DEXlactateHEMA) Nanogels for Ocular Delivery for the Treatment of DR}

\section{Hypothesis and Specific Aims}

The long-range goal of this project in chapter 3 was to develop novel poly(NIPAAm-co-DEXlactateHEMA) nanogels for long-term release of insulin across the ocular barriers for treating retinal diseases. Another advantage of choosing poly(NIPAAm-co-DEXlactateHEMA) nanogels is that they are hydrophilic in nature and offer the flexibility of aqueous loading of insulin thereby improving the drug stability. 
The immediate objective was to develop subconjunctivally injectable biodegradable nanogels with similar composition to the earlier hydrogels that can have the potential to enhance the permeability of the drugs, such as insulin, across ocular barriers and also sustain the release of drugs to treat ocular diseases like DR.

The hypothesis of this project in chapter 3, which was the sub-hypothesis 2 of the central hypothesis, was that the subconjunctivally injectable nanogels composed of DexlactateHEMA and NIPAAm can cross the ocular barriers for the potential delivery of drugs such as insulin to diabetic retinopathy.

Specific Aim 1. Prepare and characterize poly(NIPAAm-co-DEXlactateHEMA) nanogels. A series of thermoresponsive and biodegradable nanogels with different hydrophobicity, hydrophilicity, crosslinker amount, surface charge and particle size were synthesized in water.

Specific Aim 2. Assess in vitro cytotoxicity, in vitro and ex vivo permeability of the poly(NIPAAm-co-DEXlactateHEMA) nanogels. The synthesized nanogels were tested for their cytotoxicity on human fetal retinal pigmented epithelial (hfRPE cells). Ex vivo permeability of the nanogels was evaluated using in vitro BRB model of adult retinal pigmented epithelial cells (ARPE-19) grown on polyester Transwell filter. Porcine sclera and cornea were used as ex vivo ocular barriers.

\section{Material and Methods}

\section{Chemicals and Reagents}

Dextran (MW -15,000 $\mathrm{g} \cdot \mathrm{mol}^{-1}$ ) was purchased from Polysciences, Inc., Warrington, PA. The following materials were obtained from Sigma-Aldrich, Inc (St. Louis, MO): NIPAAm, 2-hydroxyl methacrylate (HEMA), 4-(N,N-diethylamino) pyridine (DMAP), N,N'-carbonyl diimidazole (CDI), L-lactide, stannous octoate (SnOct2), tetrahydrofuran (THF), dimethyl sulfoxide (DMSO), fluorescein isothiocyanate (FITC), 5-(4,6-dichlorotriazinyl) aminofluorescein (5-DTAF), FITC-dextran (MW - 70 $\mathrm{kDa}$, and $4 \mathrm{kDa}$ ), 3-(4,5-dimethylthiazol-2-yl)-2,5-diphenyltetrazolium bromide (MTT). All the chemicals were used as received. Deionized distilled water was used in all the experiments.

\section{Synthesis and Characterization}

DEX-lactateHEMA macromer with different degree of polymerization (DP) and degree of substitution (DS) was synthesized according to the previously published procedure by adjusting the feeding ratios of the corresponding precursors [423]. The structure is given in Figure 3-5. The lactide chain length (DP) of the lactate unit controls the hydrolytic degradation, and the number of lactide chains on dextran (DS) in the DEXlacateHEMA macromer controls the degree of crosslinking (swelling) in the nanogel, respectively. The DP and DS of the macromer were calculated based on the $1^{\mathrm{H}} \mathrm{NMR}$ 


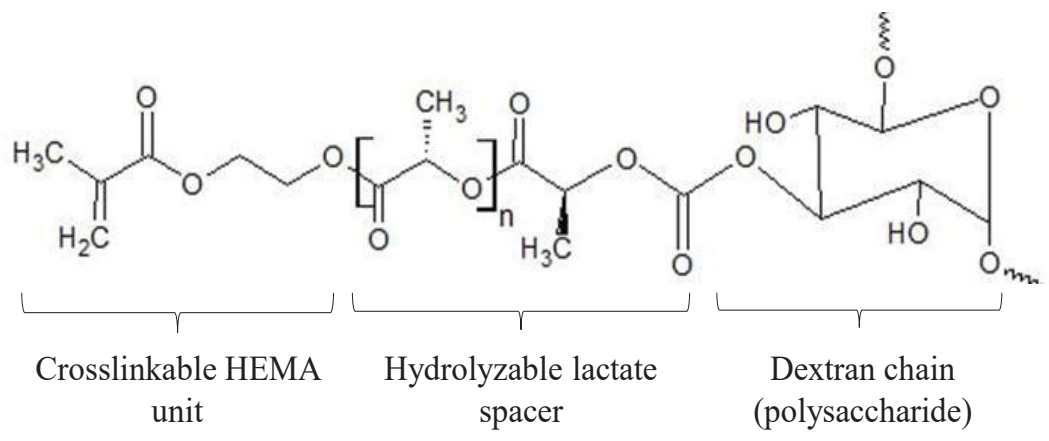

Figure 3-5. The structure of dextran macromer (DEX-LacateHEMA). 
spectra. In present work, the DEX-LactateHEMA macromer of DP/DS - 5/5.5 was synthesized and used in the preparation of nanogels.

Poly(NIPAAm-co-DEXlactateHEMA) nanogels were synthesized by UVinitiated free radical emulsion polymerization method. A prepolymer solution was prepared by dissolving NIPAAm/DEXlactateHEMA at different weight ratios: 7/2, and $6 / 3$ in DI water while stirring in $\mathrm{RBF}$ at $200 \mathrm{rpm}$ at $45^{\circ} \mathrm{C}$ with nitrogen bubbling. Later, $10 \mathrm{~mol} \%$ (with respect to NIPAAm) of anionic charge modifier acrylic acid (AA) was added to the prepolymer solution. The UV initiator $0.066 \% \mathrm{wt} \%$ Irgacure 2959 dissolved in DI water was added dropwise, and then the mixture was exposed to UV light at 1 $\mathrm{W} / \mathrm{cm}^{2}$ for $1 \mathrm{~h}$ under constant stirring at $200 \mathrm{rpm}$ (at $45^{\circ} \mathrm{C}$ and $\mathrm{N}_{2}$ purging). The scheme of synthesis is given in Figure 3-6. The obtained emulsion was cooled down to room temperature and dialyzed against the large (10-fold) volume of DI water for $4 \mathrm{~h}$ using 50 $\mathrm{kDa}$ molecular cutoff cellulose dialysis tubes. The reason to use short $4 \mathrm{~h}$ for the dialysis was to limit the nanogel degradation during the dialysis process. The purified nanogel product was then freeze dried, and FTIR confirmed the synthesis.

DTAF-labeled nanogels were prepared as follows. DTAF $(10 \mathrm{mg})$ and the nanogels $(250 \mathrm{mg})$ were dissolved in DMSO $(0.3 \mathrm{~mL})$ and sodium carbonate buffer $(0.1$ $\left.\mathrm{mol} \cdot \mathrm{L}^{-1}, 5 \mathrm{~mL}, \mathrm{pH} 9\right)$, respectively. The DTAF solution was added dropwise into the nanoparticle solutions with stirring. The reaction was carried out overnight at $4{ }^{\circ} \mathrm{C}$. The final solution was dialyzed with MWCO $50 \mathrm{kDa}$ membrane against deionized water while changing the outer water every $8 \mathrm{~h}$ for $24 \mathrm{~h}$ and freeze-dried.

The hydrodynamic size and zeta potential of the nanogles were measured at a concentration of $200 \mu \mathrm{g} \cdot \mathrm{mL}^{-1}$ in PBS at $37^{\circ} \mathrm{C}$ using dynamic light scattering (DLS) (Zetasizer NS, Malvern, United Kingdom). An average was taken from three measurements.

\section{Cell Culture}

Human-fetal retinal pigment epithelial cells (hfRPE) and adult retinal pigmented epithelium cells were seeded in T25 flasks at a density of 8000-12,000 cells cm2, and cultured in Dulbecco's Modified Eagle Medium (DMEM-F12) containing 10\% fetal bovine serum (FBS), vitamins, essential amino acids, and penicillin/streptomycin at $37 \mathrm{C}$ with $95 \%$ humidity and 5\% CO2. The medium was changed every other day. The cells were harvested with trypsin $(0.05 \%$ trypsin with $0.4 \mathrm{mM}$ EDTA) when they were $80 \%$ confluent.

\section{In Vitro Cytotoxicity of the Nanogels}

Cytotoxicity of the nanogels to hfRPE cells was examined by an MTT cell viability assay. The cells were seeded at a density of 7,500 cells per well in a 96-well plate containing $150 \mu \mathrm{L}$ media at $37 \mathrm{C}$ for $24 \mathrm{~h}$. The nanogels and the degradants after

one week of incubation of nanogels in the medium at $37^{\circ} \mathrm{C}$ were then incubated with the cells for $48 \mathrm{~h}$. Wells containing cells alone were used as a control. $20 \mu \mathrm{L}$ of MTT 


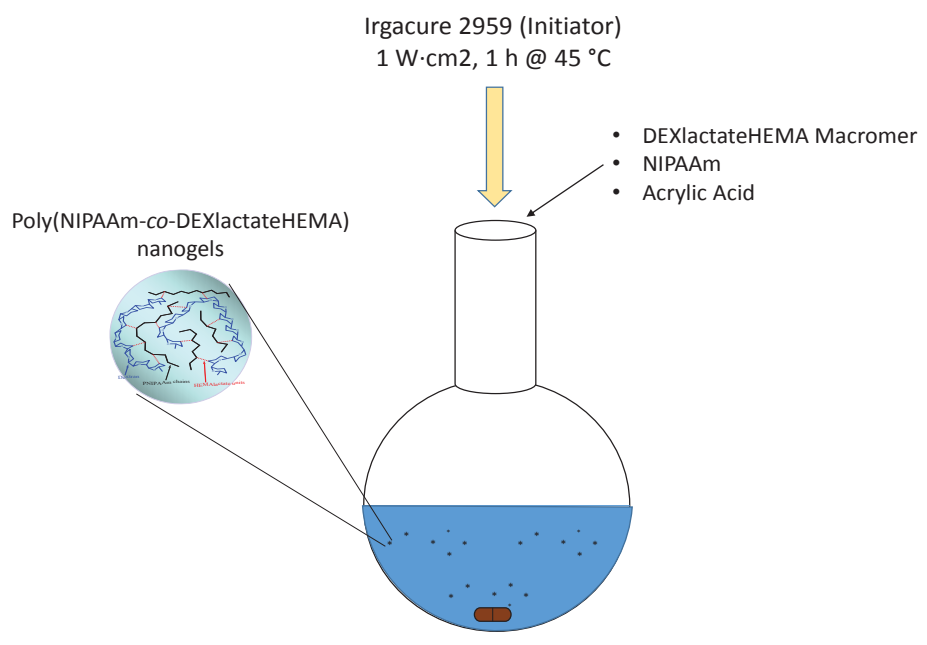

Figure 3-6. The synthesis scheme of poly(NIPAAm-co-DEXlactateHEMA) nanogels.

Notes: A prepolymer solution in DI water was exposed to UV light while stirring in RBF at $200 \mathrm{rpm}$ at $45^{\circ} \mathrm{C}$ under nitrogen bubbling. Poly(NIPAAm-co-DEXlactateHEMA) nanogel structure reprinted with permission. Huang, X., et al., Novel nanogels with both thermoresponsive and hydrolytically degradable properties. Macromolecules, 2008. 41(22): p. 8339-8345. (C) 2008 American Chemical Society. 
solution (5 mg MTT/mL PBS) was added to each well and incubated for $4 \mathrm{~h}$ at $37^{\circ} \mathrm{C}$ and $5 \% \mathrm{CO} 2$. The medium was then removed completely from the wells without disturbing the cells, and $150 \mu \mathrm{L}$ of DMSO was added to each well. The plate was covered with foil and placed on the rocking platform for 15 mins to dissolve the formazan crystals in dimethyl sulfoxide. The absorbance of the reduced form of the MTT was measured at $570 \mathrm{~nm}$ using a microplate reader ((BioTek Instruments, Inc., Winooski, VT, USA) with background subtraction. The sample and control samples were measured in quadruplicate, and the relative cell viability (\%) was calculated as percentage absorbance of the samples relative to the absorbance of the untreated control cells.

\section{In Vitro Permeability across ARPE-19 Monolayers}

Confluent ARPE-19 monolayers were constructed on coated polyester Transwell inserts (24 well-plate, $0.4 \mu \mathrm{m}$ pore size). Briefly, ARPE-19 cells were seeded on the insert at 50,000 cells $\cdot \mathrm{cm}-2$ and grown at $37^{\circ} \mathrm{C}$ with $95 \%$ humidity and $5 \% \mathrm{CO} 2$ in a cell culture incubator. The medium was changed as usual until the transepithelial electrical resistance (TEER) values reached the plateau to get the confluence ARPE-19 monolayer as the in-vitro RPE model (Figure 3-7).

DTAF-labeled nanogels or FITC-dextran control $(\mathrm{Mw}=70 \mathrm{kDa})$ was dispersed or dissolved in the culture medium and added to the apical chamber of each well at $1 \mathrm{mg} \cdot \mathrm{ml}^{-}$ 1. The transport experiments of nanogels from the apical to the basal direction at $37^{\circ} \mathrm{C}$ was conducted for four hours. At selected time points $(0,15,30,60,90,120,150,180$, $210,240 \mathrm{~min}), 30 \mu \mathrm{L}$ of the medium was sampled from the basal/receiver chamber and replaced with $30 \mu \mathrm{L}$ of fresh medium. In the end, $30 \mu \mathrm{L}$ media was sampled from both the apical and basolateral chambers. The fluorescence of the aliquots was quantified by using microplate reader (BioTek Instruments, Inc., Winooski, VT, USA) at $430 \mathrm{~nm}$ of excitation and $530 \mathrm{~nm}$ of emission wavelengths. The permeability (P0) of the components across the ARPE-19 monolayer was calculated as below [38, 424]:

The flux of the DTAF-nanogels or control FITC-dextran from the apical side of the tissue layer to the basolateral direction was measured. "Flux is defined as the amount of solute crossing a plane of the unit surface area, which is normal (perpendicular) to the direction of transport, per unit time" [425]. The apparent flux $(\mathrm{J})$ or permeation rate was calculated from the slope $\left(\Delta F_{r} / \Delta t\right)$ of the linear region of the plot of the cumulative fluorescence $\left(F_{r}\right)$ of the permeant FITC-4k dextran transported across the membrane into the receptor chamber versus time $(\mathrm{t})$ divided by the effective diffusion surface area $\left(A_{d}\right)$. The apparent permeability coefficient (P0) was calculated based on the flux of the system (J) divided by the concentration (fluorescence) difference between the donor and receiver chamber (Equation 3-1). Typically, the concentration (fluorescence) in the receiver chamber is significantly smaller than the donor chamber, the concentration of the receiver chamber is considered as zero, and the concertation in the donor chamber is obtained by dividing donor cell fluorescence $\left(F_{d}\right)$ with the volume of the donor chamber $\left(V_{d}\right)$.

$$
\text { Permability Coefficient }\left(P_{0}\right)=\frac{\text { Flux }(\mathrm{J})}{\text { Donor Concentration }}=\frac{\left(\Delta F_{r} / \Delta t\right) / A_{d}}{F_{d} / V_{d}}
$$




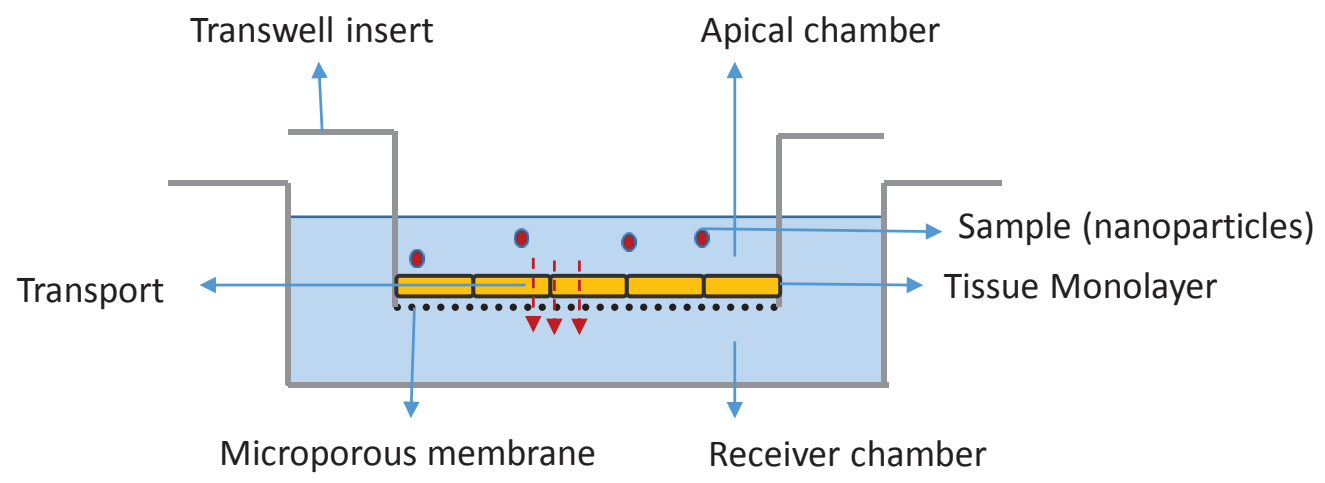

Figure 3-7. Experimental set-up for in vitro permeability.

Notes: After the formation of an ARPE-19 monolayer on the porous transwell inserts, the nanogels suspension was added to the apical chamber and the medium from the receiver chamber was sampled at selected time points and analyzed for the nanogels. The experiment was conducted at $37^{\circ} \mathrm{C}$. 


\section{AFM Measurements}

After the in vitro permeability experiment, the medium from the receiver chamber was collected and was dialyzed against DI water for $4 \mathrm{~h}$ and freeze-dried. To confirm the permeation of the nanoparticles, the freeze-dried contents from the receiving chamber was investigated for the presence of particles by using Atomic Force Microscopy.

\section{Ex Vivo Permeability across Porcine Ocular Tissues}

Porcine sclera and corneal tissues were separated from the freshly isolated porcine eyeballs within two hours are prepared for the ex vivo permeability experiments immediately. The tissues were carefully mounted in the side-by-side perfusion devices (Figure 3-8). Side-by-side diffusion cells are known as Valia-Chien Cell apparatuses (Permegear, Inc., Hellertown, PA, USA) were used to conduct the permeability study. The apparatus consisted of two glass chambers; each had an outlet for sampling and a port for heater circulator. The membranes isolated were placed in the spherical joints of Valia-Chien Cell apparatuses, and a clamp is placed on the spherical joints to hold the membrane in place. Immediately after the tissue was clamped, both chambers were filled with Glutathione Bicarbonate Ringer's Solution ( $\mathrm{pH}$ 7.2-7.4). The solution in each chamber was gently stirred using a small Teflon-coated magnetic stir bar for the homogenous distribution of the solutes. The FITC-dextran/DTAF nanogels was dissolved in the media for the ocular tissues and added to the donor chamber at $1 \mathrm{mg} \cdot \mathrm{mL}^{-1}$. The chambers were maintained at $34^{\circ} \mathrm{C}$ by circulating warm water through them. Passive transport of the FITC-dextran across the sclera and cornea tissues was carried out at $34^{\circ} \mathrm{C}$ over $4 \mathrm{~h}$ period of time. $200 \mu \mathrm{L}$ of the samples was taken out from the receptor chamber as a function of time and replaced with $200 \mu \mathrm{L}$ of fresh medium. The TEER values of the ocular tissues were monitored through the whole permeability studies to check the integrity of the membrane. The fluorescence intensities in the solutions sampled from the donor and receptor chambers were quantified using a fluorescence microplate reader (BioTek Instruments, Inc., Winooski, VT, USA) and the permeability (P0) of the samples across the sclera and cornea tissues were calculated as described earlier.

\section{Results and Discussion}

\section{Synthesis and Characterization of Nanogels}

Previously, Huang et. al from our group has published a report [426] on the synthesis of a series of poly(NIPAAm-co-DEXlactateHEMA) nanogels via thermoinitiated polymerization from DEXlactateHEMA macromer and NIPAAm monomers at various feeding ratios of the macromer and NIPAAm. In the earlier synthesis method, a high temperature $\left(70^{\circ} \mathrm{C}\right)$ and long reaction time were used for the synthesis of nanogels. However, use of such harsh conditions may not be suitable for loading of protein drugs as they may get denatured at high temperatures. To avoid such harsh conditions, in the current synthesis procedure a slightly modified technique was used by shifting from the thermo-initiated polymerization to a milder UV initiated photo emulsion polymerization using Irgacure 2959 as an initiator instead of KPS. This procedure offered the flexibility 




Figure 3-8. Experimental set-up for ex vivo permeability.

Notes: After mounting the freshly isolated porcine ocular tissue (sclera/cornea), the medium was added to both the chambers. The DTAF-nanogels suspension was added to donor chamber, and the medium from the receiver chamber was sampled at selected time points and analyzed for the fluorescence. 
of fabricating the poly(NIPAAm-co-DEXlactateHEMA) nanogels under mild conditions such as low temperature and short reaction, aiming at in situ aqueous loading of protein drugs (insulin) for ocular drug delivery. Also, the initiator Irgacure 2959 solution has been widely used for in situ polymerization in vivo and does not cause eye irritation according to the MSDS [408].

Table 3-3 shows the average hydrodynamic radii and zeta potential of the nanogels in PBS at $37^{\circ} \mathrm{C}$ for two types of nanogels synthesized (72A and 62A). The size of the nanogels was within 70-87 nm, and no significant difference was observed between two types of nanogels. Both the nanogels showed a zeta potential around -10 $\mathrm{mV}$ and the negative zeta potential observed on the nanogels could be attributed to the ionization of carboxylic acid groups of the acrylic acid introduced into the nanogels at $\mathrm{pH}$ 7.4 , as well as any carboxylic groups resulted due to partial degradation of ester bonds of lactide units. Similar to particle size, no significant difference was observed in zeta potential among the two nanogels. Prior studies in the literature have suggested that charged polymers (both cationic and anionic charge) could potentially interact with tissue surface proteins such as mucins through hydrogen bonding or other electrostatic interactions and render them mucoadhesive [427]. Also, it is reported that the presence of carboxylic functional groups in the nanoparticles helps in the permeability by various cellular internalization mechanisms [428, 429]. Because of these advantages, the charged component with a carboxylic functional group (acrylic acid) was incorporated into the nanogels. Another rationale for adding carboxylic acid groups is that functional moieties like targeting ligand and drugs could be easily conjugated to the nanogels.

As the nanogels are intended for ocular delivery, the cytotoxicity of these nanogels to hfRPE cells (part of the BRB) was tested using MTT assay. MTT assay is the most widely used colorimetric technique to check the cell viability in vitro [408]. The underlying principle of MTT assay is that mitochondrial reductase in viable cells with active metabolism convert the tetrazolium into a purple colored formazan product which shows absorbance near $570 \mathrm{~nm}$. Therefore, any metabolic dysfunction in the cells caused by toxic substances leads to reduced activity of the mitochondrial enzymes which in turn decreases the conversion of MTT to formazan. The cell viability is expressed as the percent relative absorbance of the treated cells to the untreated control cells. In vitro cytotoxicity studies of the synthesized nanogles showed that no matter whether the nanogels (72A and 63A) were incubated with the hfRPE cells directly for $48 \mathrm{~h}$ or they were degraded in the culture medium for one week and then the cells were exposed to the degraded solution (72A-D, and 63A-D) for $48 \mathrm{~h}$, the viability of the treated cells was close to $100 \%$ of control values at concentrations $0.1,0.5$, and $1 \mathrm{mg} \cdot \mathrm{ml}^{-1}$ (Figure 3-9). These results indicated that both the nanogels and their degradation products are nontoxic to cultured hfRPE for at least $48 \mathrm{~h}$, and the nanogels have the potential for further in vivo evaluation.

\section{In Vitro Permeability of Nanogels}

In order to test the nanogels for potential ocular delivery applications, an in vitro ARPE-19 monolayer was constructed on Transwell interest as the in vitro blood-ocular 
Table 3-3. Composition, particle size and zeta potential of the nanogels.

\begin{tabular}{lcccc}
\hline Notation & $\begin{array}{c}\text { NIPAAm/ } \\
\text { DEXlactateHEMA } \\
\text { (w/w ratio) }\end{array}$ & $\begin{array}{c}\text { Charge modifier } \\
\text { (acrylic acid/AA) } \\
\mathbf{1 0 ~ m o l \% ~ w r t ~} \\
\text { NIPAAm }\end{array}$ & $\begin{array}{c}\text { Size } \\
(\mathbf{n m})\end{array}$ & $\begin{array}{c}\text { Zeta } \\
\text { potential } \\
(\mathbf{m v})\end{array}$ \\
\hline $72 \mathrm{~A}$ & $7 / 2$ & $\mathrm{x}$ & $73.65 \pm 4.55$ & $-10.53 \pm 0.57$ \\
$63 \mathrm{~A}$ & $6 / 3$ & $\mathrm{x}$ & $83.99 \pm 3.25$ & $-10.36 \pm 0.76$ \\
\hline
\end{tabular}



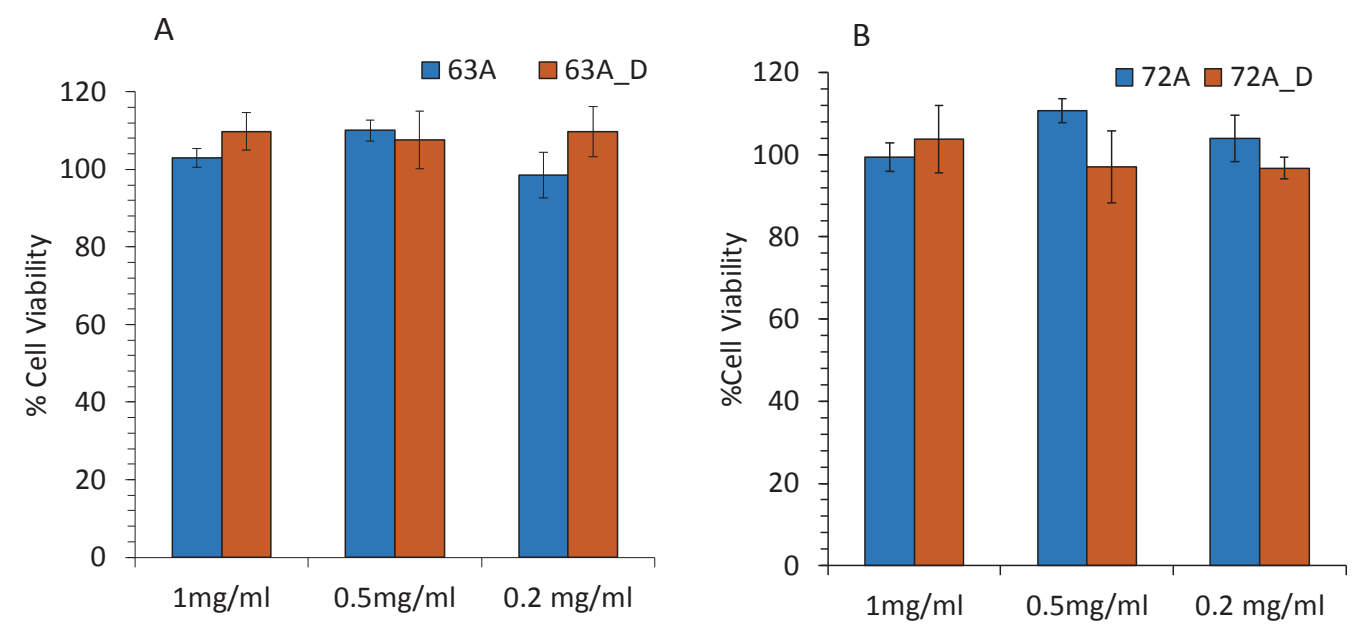

Figure 3-9. Cell viability of the nanogels to hfRPE cells, to nanogels and degradants.

Notes: The cell viability was determined by MTT assay after $48 \mathrm{~h}$ exposure to either nanogels (63A, 72A) /degradants of the nanogels (63_D, 72_D). 63A/63A_D (A) and 72A/72A_D (B). (Mean \pm SD, $n=4)$ 
barrier model. The plateau TEER value obtained for the confluent monolayer was $160 \pm 10 \mathrm{ohm} \cdot \mathrm{cm} 2$. The permeability was measured by examining the transport of DTAFlabeled nanogels from the apical to the basolateral side of the in vitro RPE monolayers. RPE monolayer is part of BRB that separates the outer surface of the neural retina from the choroid. Because of the presence of tight junctions the RPE forms a strong barrier; however, it is capable of a number of specialized transport processes. Figure 3-10A, B shows the transport and permeability across the in-vitro ARPE-19 monolayer for the two types of nanogels and dextran-70k control at $1 \mathrm{mg} \cdot \mathrm{mL}^{-1}$. Compared with the control dextran-70k, the nanogels have a significantly higher permeability ( $\geq 35$ fold) across the in vitro BRB model. Meanwhile, no statistically significant difference was observed between the two types of nanogels in terms of in vitro permeability. TEER values of the ARPE-19 monolayers before and after the permeability studies remained similar confirming the integrity of the membrane during the study. To further confirm if the detected fluorescence in the receiver chamber is from the nanogels or any leaked DTAF dye, the medium from the receptor chambers was collected to analyze for the presence of nanoparticles by AFM and the representative AFM images from the 63A receptor chamber (Figure 3-10C) confirm the presence of anionic nanogels. As the pore of the paracellular pathway between the cells is very small, because of the larger hydrodynamic size of the nanogels $(>75 \mathrm{~nm})$ in PBS ( $\mathrm{pH} 7.4)$, it is very unlikely that the nanogels can cross the in vitro BRB model by a paracellular pathway. Further, the presence of tight junction restricts the paracellular entry of solutes. Therefore, the negative zeta potential, carboxylic functional groups, nano-sized particle nature might have helped the nanogels to cross the RPE cell monolayer through transcellular route by various mechanisms such as endocytosis/exocytosis mechanisms [346, 430-432].

\section{Ex Vivo Permeability of Nanogels across Porcine Ocular Tissues}

The nanogels were further evaluated for their penetrating capacity across porcine ocular tissues (i.e. sclera, and cornea) mounted in side-by-side perfusion devices.

Figure 3-11 showed the permeability of both prototype nanogels and dextran-70k control across porcine sclera and cornea. Compared with the control dextran-70k, both 63A and $72 \mathrm{~A}$ nanogels have a significantly higher permeability (at least four-fold) across the sclera and cornea tissues. Similar to the in the in-vitro permeability study, no statistically significant difference was observed between the two types of nanogels. Because of the similar particle size and zeta potential, both $63 \mathrm{~A}$ and $72 \mathrm{~A}$ nanogels might have behaved in a similar way in terms of permeability across in vitro and ex vivo ocular barriers. Further studies needed to be conducted to understand the transport mechanisms of these nanoparticles.

\section{Permeability of Porcine Ocular Tissues at Different Stages of Development}

The prevalence of DR is more common in elderly population, and as aging causes a multitude of changes in biological membrane properties, it is important to investigate the permeability of ocular tissues at different stages of development for understanding physiological conditions of the eye and developing strategies (such as nanocarriers) to enhance drug permeability across these tissues for treating many ocular diseases. 

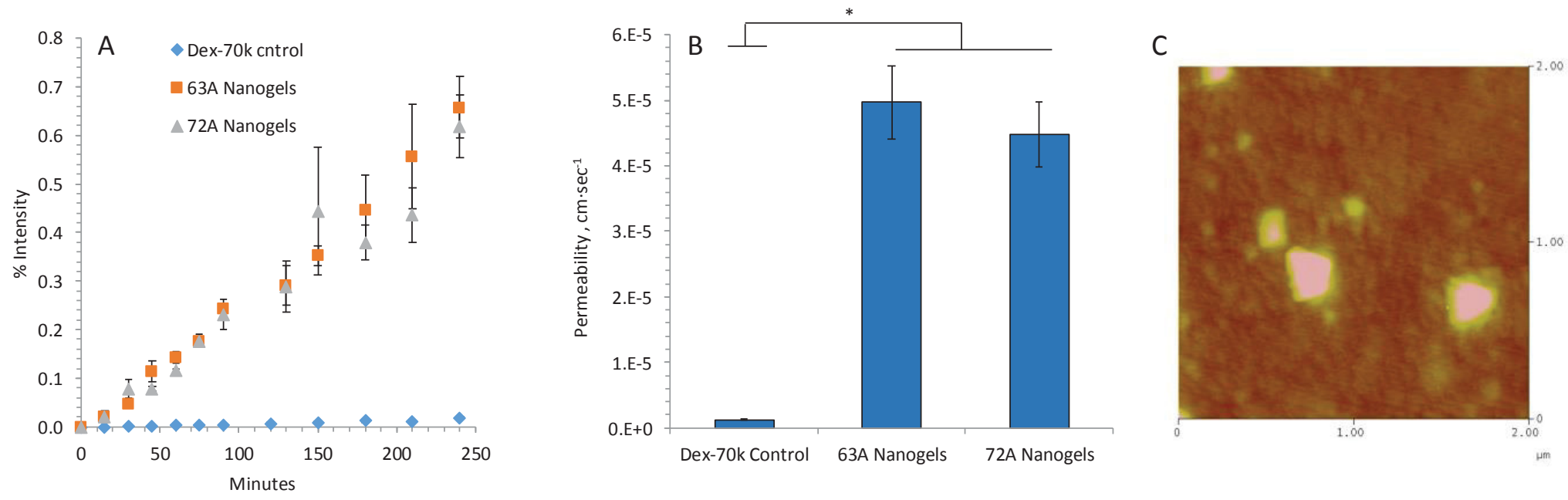

Figure 3-10. In vitro transport (A) and permeability (B) of nanogels (63A, 72A) and 70kDa-dextran across ARPE-19 cell monolayer; AFM image of the nanoparticle from the receptor chamber (C).

Notes: Nanogels showed higher (at least 35 -fold) permeability than control dextran across in vitro ARPE-19 monolayer, and the AFM results confirmed the presence of nanogels in the receiver chamber. (Mean $\pm \mathrm{SE}, \mathrm{n}=4, \mathrm{p}<0.05$ ). 



Figure 3-11. Comparison of nanogels permeability across ex vivo piglet sclera (A) and cornea (B).

Notes: Nanogels showed higher permeability (at least $\sim 4$-fold) across ex vivo porcine sclera and cornea. $(\mathrm{n}=3, \mathrm{p}<0.05)$. 
Therefore to understand the ocular permeability with aging a short study was conducted where the permeability differences of the porcine sclera and the cornea at different stages (preterm, normal term, adult) of development was examined ex vivo using a fluorescent probe FITC-dextran-4k. Figure 3-12 and Figure 3-13 illustrate the thickness and permeability of the ocular tissues, respectively. The sclera became thicker with the growth of pigs from preterm to normal-term to the adult stage, while the transscleral permeability was decreased with age. In case of the cornea, it was observed that the normal-term cornea has a similar thickness and permeability as the pre-term porcine cornea, but at the adult stage, the thickness increased and the permeability was significantly lowered. The permeability of the porcine cornea and sclera showed to be dependent on the developmental stage of the pig.

\section{Conclusions}

Poly(NIPAAm-co-DEXlactateHEMA) nanogels were successfully synthesized using photopolymerization under mild temperature. In vitro cytotoxicity evaluation of these nanogels showed that high cell viability could be retained after incubating the nanogels with hfRPE cells at up to $1 \mathrm{mg} \cdot \mathrm{ml}^{-1}$ for $48 \mathrm{~h}$, indicating that the nanogels were not toxic to the cells tested. The nanogels were highly permeable across the ARPE-19 monolayers as compared with the controls dextran-70k. Further, ex vivo permeability of the nanogels showed they were at least four-fold more permeable than the control dextran-70k. In addition, it was found that the development stages of pigs affect the permeability of ocular tissues depending on the tissue types. These results could provide insight into designing drug carriers to enhance drug permeability across ocular tissues at different development stages.

\section{Future Work}

The future work of Poly(NIPAAm-co-DEXlactateHEMA) nanogels include - i) optimization of the nanogels for loading and sustained release of insulin; ii) evaluation of in vitro and ex vivo permeability of the nanogels evaluation of insulin-loaded nanogels; iii) investigation of in vivo safety, biodistribution and efficacy of the insulin-loaded nanogels for DR. The permeability differences with age can be understood by further continuing the work to evaluate morphological properties of the membranes across different stages of development. 



Figure 3-12. Comparison of the thickness of porcine sclera (A) and cornea (B) at different stages of development.

Notes: Preterm ( 28 week preterm piglets), the normal-term (one-week old normal-term piglets), Adult Pigs (3-3.5 month old adult pigs). (Mean \pm SE, $n=4$-sclera, 3-cornea), $\mathrm{p}<$ $0.05)$. Thickens of porcine sclera was observed to be increased with increase in the age. However, in case of the cornea, no increase was observed between the preterm and normal, but the thickness of adult cornea increased significantly. 

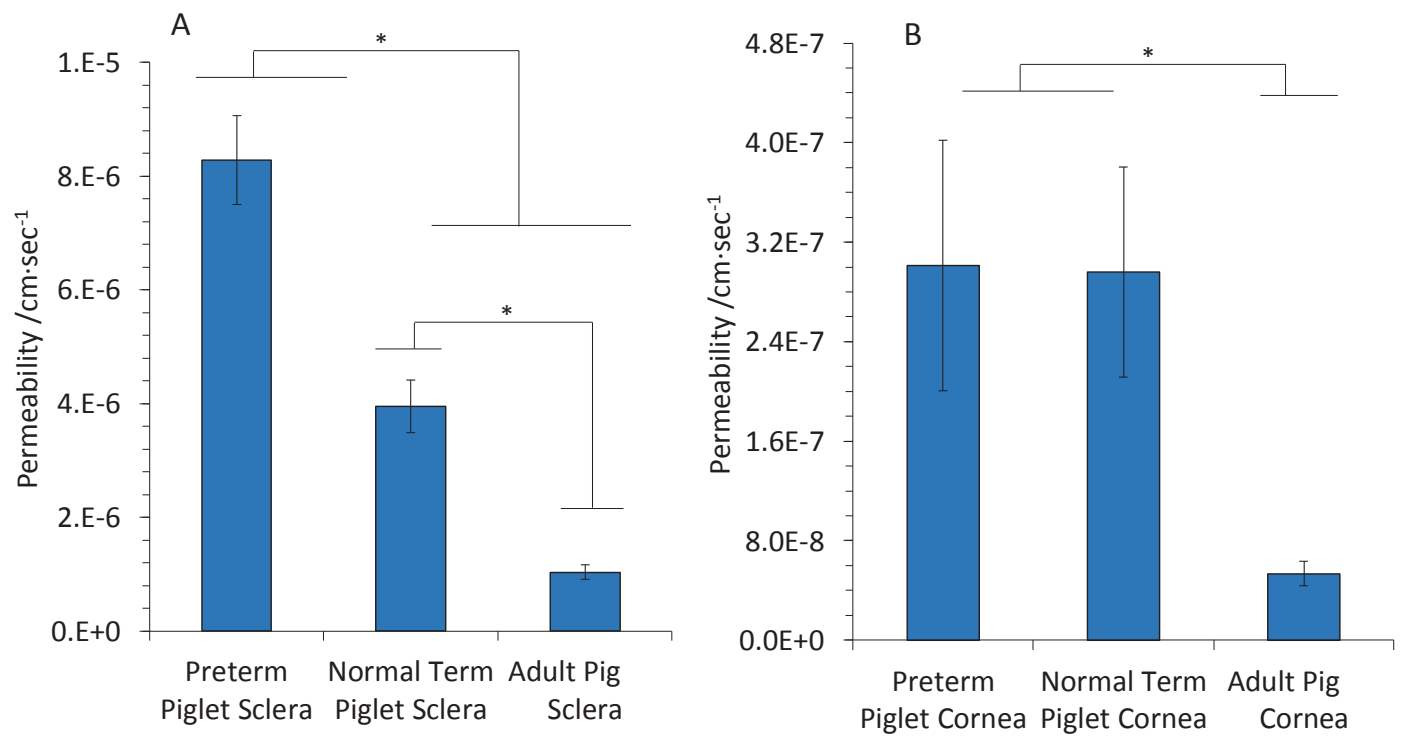

Figure 3-13. Comparison of ex vivo permeability of porcine sclera (A) and cornea (B) at different stages of development.

Notes: Preterm (28-week preterm piglets), the normal-term (one-week old normal-term piglets), Adult Pigs (3-3.5 month old adult pigs). (Mean \pm SE, $n=4$-sclera, 3-cornea; $\mathrm{p}<$ $0.05)$. The permeability of porcine sclera was observed to be decreased with increase in the age. However, in case of the cornea, the preterm and normal showed similar permeability, but the permeability of adult cornea decreased significantly. 


\section{Beta-cyclodextrin-poly( $\beta$-amino ester) (CD-p-AE) Nanoparticles as Drug Carriers to Enhance BBB Permeability of 17-AAG to Treat AD}

\section{Hypothesis and Specific Aims}

Our group has developed and characterized CD-p-AE nanoparticles for the potential application of drug delivery across BBB [50]. Incorporation of $\beta$-cyclodextrin $(\beta-C D)$ into the nanoparticle structure facilitates the loading of hydrophobic drugs and thereby expected to improve the solubility of the hydrophobic drugs. Earlier reported studies showed that CD-p-AE nanoparticles were nontoxic, hydrolytically degradable, very permeable to the in vitro $\mathrm{BBB}$, and sustained the release of a hydrophobic drug doxorubicin. The long-range goal of this project in chapter 3 was to develop 17-AAG loaded CD-p-AE nanoparticles for AD treatment. The immediate objective was to investigate the feasibility of biodegradable $\beta$-CD-based nanoparticles containing tertiary amine groups for enhancing the BBB permeability and efficacy of 17-AAG in AD treatment.

The hypothesis of this project in chapter 3, which was the sub-hypothesis 3 of the overall hypothesis of the dissertation, was that the nanoparticles composed of $\beta$ cyclodextrin and poly( $\beta$-amino ester) segments can enhance the solubility and BBB permeability of Hsp90 inhibitors such as 17-AAG to treat Alzheimer's disease.

Specific Aim 1: Prepare and evaluate in vitro drug release of 7-AAG-loaded $\beta$ cyclodextrin-poly( $\beta$-amino ester) nanoparticles. CD-p-AE nanoparticles were synthesized through Michael addition of acrylated $\beta$-CD macromer and 1,4-butanediol diacrylate with an amine molecule such as N, N-dimethylethyldiamine. 17-AAG was loaded into the nanoparticles, and the release studies were carried out in PBS (pH 7.4) at $37^{\circ} \mathrm{C}$.

Specific Aim 2: Evaluate in vivo efficacy of 17-AAG-loaded $\beta$-cyclodextrinpoly( $\beta$-amino ester) nanoparticles. The 17-AAG-loaded CD-p-AE nanoparticles were injected into mice through iv route, and the drug levels in the brain and activation of Hsp70 was studied and compared to the drug alone.

\section{Material and Methods}

\section{Chemicals and Reagents}

The following materials were obtained from Sigma-Aldrich, Inc., St. Louis, MO: $\beta$ - CD, acryloyl chloride (96\%), anhydrous 1-methyl-2-pyrrolidinone (NMP), dimethyl sulfoxide-d ${ }_{6}\left(\right.$ DMSO-d $\left._{6}, 99.9 \%\right), 1,4$-butanediol diacrylate, N,N-dimethyl ethylenediamine, chloroform, ethyl acetate, phosphate buffered saline (PBS, pH 7.4), N,N-dimethylformamide (DMF, HPLC grade), DTAF. 17-AAG was obtained from LC Laboratories. All these chemicals were used as received without further purification 
unless otherwise noted. Dialysis membrane (molecular weight cutoff (MWCO)

12000-14000 Da) was bought from Spectrum Laboratories. Polyester Transwell inserts (24 well-plate, $0.4 \mu \mathrm{m}$ pore size) were obtained from Costar (Cambridge, MA).

\section{Preparation of 17-AAG-loaded CD-p-AE Nanoparticles}

CD-p-AE nanoparticles were synthesized according to the Michael addition of Acrylated CD Macromer and 1,4-butanediol diacrylate with an amine N, Ndimethylethyldiamine by following previously published procedure [50]. The synthesized CD-p-AE nanoparticles were confirmed by using 1H-NMR. Five parts of CD-p-AE nanoparticles was dissolved with 1 part of 17-AAG in ethanol and incubated overnight at room temperature. The resulting solution was diluted with water and freeze dried to obtain the 17-AAG loaded nanoparticle. The size and zeta potential of the nanoparticles was measured by using Malvern Zetasizer.

\section{7-AAG In Vitro Release}

The release of 17-AAG from CD-p-AE nanoparticles was conducted in PBS ( $\mathrm{pH}$ 7.4) at $37^{\circ} \mathrm{C}$ using the dialysis method. Briefly, 17-AAG loaded CD-p-AE nanoparticles were dispersed in PBS ( $\mathrm{pH} 7.4$ ) at $1 \mathrm{mg} \cdot \mathrm{ml}^{-1}$, and then loaded into dialysis membrane tubing (MWCO $12-14 \mathrm{kDa})$. The sealed membrane tubing was immersed into PBS (pH 7.4) release medium $(400 \mathrm{~mL})$ in a tube at $37^{\circ} \mathrm{C}$. At each scheduled time point, entire release medium was replaced with the fresh medium to maintain the sink conditions. The sampled release medium was analyzed for 17-AAG by using LC-MS/MS.

\section{In Vivo Studies of 17-AAG-loaded CD-p-AE Nanoparticles}

To investigate the efficiency of nanoparticles in enhancing the BBB permeability of 17-AAG, a study was conducted where a group of wild-type C57BL/6 was injected with 17-AAG alone and 17-AAG loaded CD-p-AE nanoparticles at a dose equivalent to 5 $\mathrm{mg}$ drug. After 24 hours of injection, the mice were sacrificed, and the brain samples were isolated to study the concentration of 17-AAG in the brain. The brain tissue was placed in cryopreservation vials and preserved by snap freezing using liquid nitrogen, and stored at $80^{\circ} \mathrm{C}$ until analysis. Brain tissues were homogenized in water $(1: 2$, tissue: buffer) prior to analysis using LCMS [15]. To evaluate the efficiency of drug loaded nanoparticles in improving the efficacy of 17-AAG, another group of wild-type C57BL/6 mice were selected and injected with 17-AAG alone and 17-AAG loaded CD-p-AE nanoparticles at a dose equivalent to 2.5 and $5 \mathrm{mg}$ drug. The mice were sacrificed post 24 $\mathrm{h}$ injection, and a western blot analysis was performed according to the previously described procedure to study the expression of Hsp70 in the brain [433]. The drug was extracted using protein precipitation by $\mathrm{ACN}$, and the reconstituted samples were analyzed by LC-MS/MS. LC was performed on a UPLC system (Nexera XR, Shimadzu Corporation) consisting of a binary pump, column oven, degasser units, and autosampler units. $10 \mu \mathrm{L}$ of sample extracts were injected into the reversed-phase Luna C18 (2) column $(50 \times 2.0 \mathrm{~mm}$ i.d., $3 \mu$; Phenomenex, Torrance, CA, USA). Acetonitrile/water mixture $(70 / 30, \mathrm{v} / \mathrm{v})$ containing $0.01 \%$ formic acid was used as a mobile phase and was 
pumped at a flow rate of $0.3 \mathrm{~mL} \cdot \mathrm{min}-1$. Total run time was $4.5 \mathrm{~min}$ for each sample injection. The column temperature was kept at $25^{\circ} \mathrm{C}$. The LC elute was introduced into the Applied Biosystem Triple QuadTM 5500, a triple-quadrupole tandem mass spectrometer equipped with turbo spray ionization source, for quantification of the analytes in negative ion mode (ESI + ). Detection of the analyte ions was performed in a multiple reaction monitoring modes and transitions of $\mathrm{m} / \mathrm{z} 584.4 \rightarrow 305.2$, and $615.3 \rightarrow$ 474.4 were used for LNG and 17-DMAG (IS), respectively. Analyst ${ }^{\circledR} 1.6 .2$ software was $^{-1}$ used for processing the data. The detailed experimental conditions are: ion spray voltage $4500 \mathrm{v}$, collision gas $7 \mathrm{psi}$, temperature $550 \circ \mathrm{C}$, collision energy $-38 \mathrm{v}$, entrance potential $-10 \mathrm{v}$, collision exit potential $-17 \mathrm{v}$, declustering potential $-15 \mathrm{v}$, curtain gas $20 \mathrm{psi}$, ion spray gas I 40 psi and ion spray gas II 40 psi.

\section{Results and Discussion}

\section{7-AAG Loading and Release from CD-p-AE Nanoparticles}

Our collaborators work on 17-AAG showed that it was effective in attenuating the synaptic toxicity induced by soluble $A \beta$ and memory impairment in mice [433]. However, 17AAG has poor water-solubility and blood-brain barrier permeability, which are potential problems for clinical construction and hurdles for its clinical application [434]. Preliminary results from earlier studies by Gil ES et. al from our group demonstrated that CD-p-AE nanoparticles do not affect the integrity of the in vitro $\mathrm{BBB}$ models, and the nanoparticles have much higher permeability than dextran control across the in vitro $\mathrm{BBB}$ models [50]. Therefore, CD-p-AE nanoparticles were selected to investigate the feasibility of using this system as a drug carrier for improving the solubility and permeability of the 17-AAG for AD treatment. The CD-p-AE nanoparticles were synthesized according to the previously published procedure [50], and 17-AAG was loaded at $16.67 \%$. The average hydrodynamic size and zeta of the CD-p-AE nanoparticles were $74.01 \pm 1.7 \mathrm{~nm}$ and $+14.5 \pm 1.04 \mathrm{mv}$, respectively. Solubility testing studies demonstrated that loading of 17-AAG into the CD-p-AE has enhanced the solubility of the drug by at least 20 times. Beta-cyclodextrin is known to increase the solubility of the hydrophobic drugs by their ability to form reversible inclusion complexes with the drugs by taking the drug moieties into the central cavity of the cyclodextrin by displacing water [435]. This complexation effectively encapsulates the drugs within the cyclodextrin, rendering them water-soluble [435].

Figure 3-14 shows that release of 17-AAG from the CD-p-AE nanoparticles. The initial burst release during first $24 \mathrm{~h}$ could be attributed to any excess or free surface drug that was not loaded into nanoparticles. After the initial burst, a slow drug release was observed for at least 8 days. Earlier reported studies showed that the CD-p-AE nanoparticles showed a gradual degradation for more than one month, and this slow degradation rate might contribute to the long release time for 17-AAG. Additionally, the favorable hydrophobic interaction between 17-AAG and CD-p-AE nanoparticles might be strong enough to limit the dissociation of 17-AAG from the nanoparticles leading to the sustained 17-AAG release [50]. 


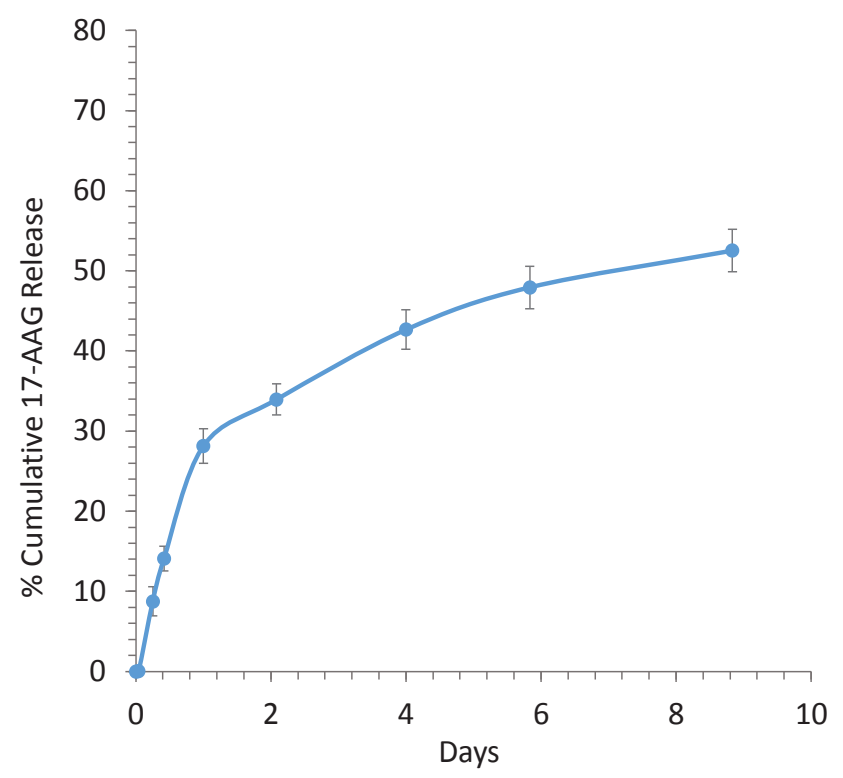

Figure 3-14. In vitro release of 17-AAG from CD-p-AE nanoparticles.

Notes: CD-p-AE nanoparticles released about $30 \%$ of the drug in the initial 24 hours, followed by a sustained release for at least 9 days. Data represent the mean $\pm \operatorname{SD}(n=3)$. 


\section{In Vivo Studies of 17-AAG-loaded CD-p-AE Nanoparticles}

Earlier studies on in vitro permeability of CD-p-AE nanoparticles showed that the nanoparticles were very permeable ( $\geq 1.6$ fold) to the in vitro BBB models (BBMVEC and HBMVEC monolayers) compared to the dextran control, and the results suggested that the nanoparticles have a high chance of delivering drugs across BBB. Therefore, to demonstrate the ability of CD-p-AE nanoparticles in improving the permeability of 17AAG, a preliminary in vivo study was conducted where the 17-AAG-loaded nanoparticles and 17-AAG alone in 5\% DMA were injected intravenously into the mice (dose $5 \mathrm{mg} \cdot \mathrm{kg}^{-1}$ ) and the brain tissues concentrations of the drug were estimated after 24 hr. The results showed that CD-p-AE nanoparticles were able to achieve 2-fold higher brain concentration of 17-AAG compared to the drug alone (Figure 3-15). Also, an efficacy study was conducted where induction of heat shock response by 17-AAG alone/ 17-AAG-loaded nanoparticles $\left(5 \& 2.5 \mathrm{mg} \cdot \mathrm{kg}^{-1}\right.$ ) was measured by estimating the levels of Hsp70 in the cortex. Results demonstrated that 17-AAG conjugated with nanoparticles induced more heat shock response compared with 17-AAG alone, as evidenced by increased Hsp70 protein level (Figure 3-16). This enhanced activity could be attributed to the increased drug levels in the brain. The data suggested that CD-p-AE nanoparticles did increase the accumulation and efficacy of 17-AAG in the brain, which might result from the fact that the nanoparticles enhanced the permeability of 17-AAG through the $\mathrm{BBB}$. The presence of cationic polyamine group and $\beta-\mathrm{CD}$ might have helped the nanoparticles to cross the $\mathrm{BB}$ through carrier-mediated transport [87]. About the transport mechanism of CD-p-AE nanoparticles across the BBB, the assumption is that adsorptive-mediated transcytosis (AMT) may be involved in the process because of favorable electrostatic interactions between the positive surface charge of CD-p-AE nanoparticles and negative charges on the endothelial surface of the BBB $[88,436]$. However, further studies are needed to confirm these assumptions.

\section{Conclusions}

CD-p-AE nanoparticles were synthesized and loaded with 17-AAG. Incorporation of 17-AAG into the nanoparticle structure has allowed improving the water solubility of the drug. CD-p-AE nanoparticles sustained the release of 17-AAG for at least one week in vitro. Crosslinking of $\beta-\mathrm{CD}$ with poly-AE enabled the fabrication of promising $\mathrm{CD}-\mathrm{p}$ AE nanoparticles that showed increased permeability of the loaded drug 17-AAG across BBB by 2-fold, and resulted in enhanced expression of the Hsp70 protein in the brain. These results suggested that the developed CD-p-AE nanoparticles can be used as potential drug carriers for transporting the drug across the $\mathrm{BBB}$ to treat chronic diseases such as AD in the brain.

\section{Future Work}

The future work of CD-p-AE nanoparticles include, a) continue optimization of the CD-p-AE nanoparticles for enhanced water solubility and sustained release of 


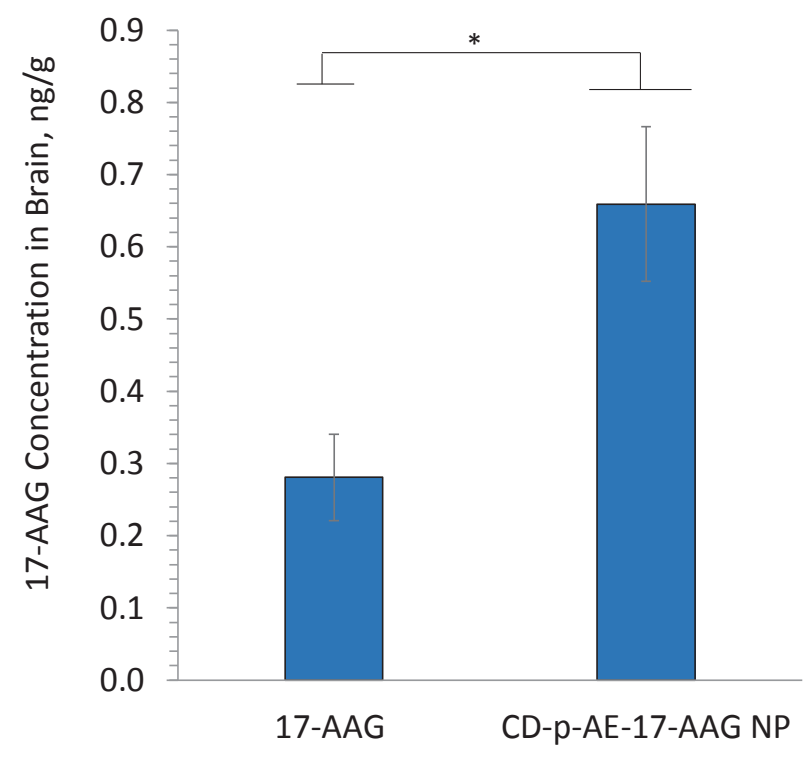

Figure 3-15. 17-AAG levels in the brain at $24 \mathrm{~h}$ following intravenous injection of 17-AAG alone/17-AAG loaded CD-p-AE nanoparticles.

Notes: Control: 17-AAG alone in 5\% DMA (filled); Sample: 17-AAG-loaded CD-p-AE nanoparticles in 5\% DMA (pattern); Injection dose: $5 \mathrm{mg} \cdot \mathrm{mL}^{-1}$ equivalent to $17-\mathrm{AAG}$. Nanoparticles resulted in $\sim 2$-fold higher 17-AAG levels in brain compared to the 17$\mathrm{AAG}$ alone. Data represent the mean $\pm \mathrm{SE}(\mathrm{n}=3 \mathrm{p}<0.05)$. 


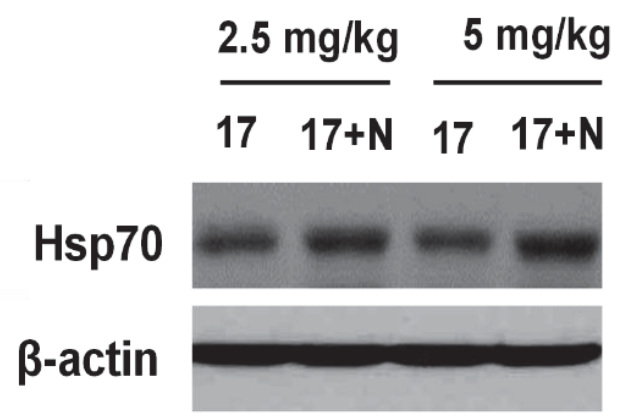

Figure 3-16. Hsp70 levels in the brain at $24 \mathrm{~h}$ following intravenous injection of 17AAG alone/17-AAG-loaded CD-p-AE nanoparticles.

Notes: 17: 17-AAG in 5\% DMA; 17+N: 17-AAG-loaded CD-p-AE nanoparticles in 5\% DMA. Injection doses: 2.5 and $5 \mathrm{mg} \cdot \mathrm{mL}^{-1}$ equivalent to 17-AAG. Nanoparticles resulted in higher expression of Hsp 70 levels in cortex compared to the 17-AAG alone. 
17-AAG; c) Modification by active targeting to direct more socially to the target site and minimizing the exposure to other tissues; b) Assessment of in vitro bioeffects, in vivo safety, biodistribution, pharmacokinetics and bioeffects of systemically injected 17AAG-loaded CD-p-AE nanoparticles. 


\section{CHAPTER 4. CONCLUSIONS}

Polymer-based drug delivery systems can significantly influence the overall performance of a drug by altering various parameters such as concentration, location, and duration in vivo. In this way, it gives a promising approach to minimize the side effects, increase the overall therapeutic efficacy of the drug, and improve patient compliance and adherence. Despite the advances in using different drug delivery systems for various medical applications, still, there are certain conditions which lack effective and compliant delivery systems. One of such unmet medical needs was identified to be the affordable injectable LARCs that can provide contraception for five months to one year, and the other requirement was the need for drug carriers that can enhance the permeability of the drugs across the biological barriers and sustain the release as well.

The main objective of this research has been to develop biodegradable polymeric biomaterials in different forms for long-acting contraception and drug delivery to the eye and brain. This has been achieved by fabrication of polyester-based ISD systems for sustained release of LNG for long-acting contraception (chapter 2), and polysaccharidebased nanoparticles for sustained drug delivery across the biological barriers (chapter 3 ).

The following is the summary of the project of LNG-containing ISD systems for long-acting contraception (chapter 2):

- A simple, fast and sensitive LC/MS/MS method for quantifying levonorgestrel (LNG) in rat plasma was developed, fully validated and successfully applied to PK study of LNG injected SubQ in rats. Rat plasma of $100 \mu \mathrm{L}$, liquid-liquid extraction and LLOQ $0.5 \mathrm{ng} \cdot \mathrm{mL}^{-1}$ were used for the method development.

- An accelerated release method for the evaluation of ISD formulations that can effectively correlate the months of long-term release in a short time such as days or weeks was developed.

- The prototype formulations (64 and 96) have suppressed the rat normal estrous cycles for at least 5 months after one SubQ injection, and a rapid return of fertility was observed after the end of the treatment.

- The optimized lead formulation 96r [4\% LNG, 4\% PLGA (0.63 iv), 16\% PLA (0.47 iv), 4\% PLA (0.63 iv), 72\% NMP/TEC (9:1)] designed by unique strategy has shown favorable injectability $(23 \mathrm{G})$ and a low in vivo initial burst, and has a high chance that it can show a promising long-term in vivo LNG release.

- Overall, the results demonstrated the injectable ISD formulations can serve as an effective means of administering LNG for long-term reversible contraception suggesting that the designed ISD formulations have great potential for developing future robust and affordable injectable LARC products that could improve access to family planning around the globe.

The summary from the project of polysaccharide-based nanoparticles for sustained drug delivery across the biological barriers (chapter 3 ) are: 
- The developed dextran-based poly(NIPAAm-co-DEXlactateHEMA) nanogels were nontoxic, and were highly permeable than dextran-70 $\mathrm{kDa}$ control across the in vitro ARPE-19 monolayers ( $\sim 35$-fold), ex vivo porcine sclera and cornea (at least 4-fold).

- The developed $\beta$-CD-based CD-p-AE nanoparticles sustained the release of 17AAG in vitro for at least one week, and showed increased permeability of the loaded drug 17-AAG across BBB by 2-fold, and resulted in enhanced expression of the Hsp70 protein in the brain cortex.

- Overall, the developed nanoparticles have shown great potential for delivering the drugs to the eye and brain.

In conclusion, the hypothesis of the dissertation that different biodegradable polymeric biomaterials based on polyesters or polysaccharides can act as effective systems for long-acting contraception for five months or longer, and drug delivery to the eye and brain was accepted. While the initial objectives of this work were met, still extensive further in vitro and in vivo investigations need to be conducted with the developed biomaterials for optimization, and demonstration of the long-term therapeutic safety, efficacy; and scale-up potentials. Besides, the developed biodegradable biomaterials can be optimized to be a versatile drug delivery platform and could be employed for encapsulating other therapeutics for sustained systemic or local drug delivery. 


\section{LIST OF REFERENCES}

1. Avorn, J., The $\$ 2.6$ billion pill-methodologic and policy considerations. New England Journal of Medicine, 2015. 372(20): p. 1877-1879.

2. Estimates, M.R. and D. Cost, MSF Response to Tufts CSDD Study on Cost to Develop a New Drug. 2014.

3. Tiwari, G., et al., Drug delivery systems: An updated review. International journal of pharmaceutical investigation, 2012. 2(1): p. 2.

4. Speers, M. and C. Bonnano, Economic aspects of controlled drug delivery. Encyclopedia of Controlled Drug Delivery. New York, NY: Wiley, 1999: p. 341347.

5. Glass, G., Pharmaceutical patent challenges-time for reassessment? Nature Reviews Drug Discovery, 2004. 3(12): p. 1057-1062.

6. Panyam, J. and V. Labhasetwar, Biodegradable nanoparticles for drug and gene delivery to cells and tissue. Advanced drug delivery reviews, 2003. 55(3): p. 329347.

7. Pillai, O. and R. Panchagnula, Polymers in drug delivery. Current opinion in chemical biology, 2001. 5(4): p. 447-451.

8. Langer, R., New methods of drug delivery. Science, 1990. 249(4976): p. 15271533.

9. $\quad$ Allen, T.M. and P.R. Cullis, Drug delivery systems: entering the mainstream. Science, 2004. 303(5665): p. 1818-1822.

10. Nair, L.S. and C.T. Laurencin, Biodegradable polymers as biomaterials. Progress in polymer science, 2007. 32(8): p. 762-798.

11. Katti, D., et al., Toxicity, biodegradation and elimination of polyanhydrides. Advanced Drug Delivery Reviews, 2002. 54(7): p. 933-961.

12. Makadia, H.K. and S.J. Siegel, Poly lactic-co-glycolic acid (PLGA) as biodegradable controlled drug delivery carrier. Polymers, 2011. 3(3): p. 13771397.

13. Bala, I., S. Hariharan, and M.R. Kumar, PLGA nanoparticles in drug delivery: the state of the art. Critical Reviews ${ }^{\mathrm{TM}}$ in Therapeutic Drug Carrier Systems, 2004. 21(5).

14. Lassalle, V. and M.L. Ferreira, PLA nano-and microparticles for drug delivery: an overview of the methods of preparation. Macromolecular bioscience, 2007. 7(6): p. 767-783.

15. Anderson, J.M. and M.S. Shive, Biodegradation and biocompatibility of PLA and PLGA microspheres. Advanced drug delivery reviews, 2012. 64: p. 72-82.

16. Saini, P., M. Arora, and M.R. Kumar, Poly (lactic acid) blends in biomedical applications. Advanced Drug Delivery Reviews, 2016.

17. Lucas, N., et al., Polymer biodegradation: Mechanisms and estimation techniques-A review. Chemosphere, 2008. 73(4): p. 429-442.

18. Liu, Z., et al., Polysaccharides-based nanoparticles as drug delivery systems. Advanced drug delivery reviews, 2008. 60(15): p. 1650-1662. 
19. Janes, K., P. Calvo, and M. Alonso, Polysaccharide colloidal particles as delivery systems for macromolecules. Advanced drug delivery reviews, 2001. 47(1): p. 8397.

20. García-González, C., M. Alnaief, and I. Smirnova, Polysaccharide-based aerogels-Promising biodegradable carriers for drug delivery systems. Carbohydrate Polymers, 2011. 86(4): p. 1425-1438.

21. Bhattarai, N., J. Gunn, and M. Zhang, Chitosan-based hydrogels for controlled, localized drug delivery. Advanced drug delivery reviews, 2010. 62(1): p. 83-99.

22. Felt, O., P. Buri, and R. Gurny, Chitosan: a unique polysaccharide for drug delivery. Drug development and industrial pharmacy, 1998. 24(11): p. 979-993.

23. Jeong, B., et al., Biodegradable block copolymers as injectable drug-delivery systems. Nature, 1997. 388(6645): p. 860-862.

24. Kolybaba, M., et al. Biodegradable polymers: past, present, and future. in ASABE/CSBE North Central Intersectional Meeting. 2006. American Society of Agricultural and Biological Engineers.

25. Farokhzad, O.C. and R. Langer, Impact of nanotechnology on drug delivery. ACS nano, 2009. 3(1): p. 16-20.

26. Rosen, H. and T. Abribat, The rise and rise of drug delivery. Nature Reviews Drug Discovery, 2005. 4(5): p. 381-385.

27. Moses, M.A., H. Brem, and R. Langer, Advancing the field of drug delivery: taking aim at cancer. Cancer cell, 2003. 4(5): p. 337-341.

28. Frank, R.G., The ongoing regulation of generic drugs. New England Journal of Medicine, 2007. 357(20): p. 1993-1996.

29. Angelova, N. and D. Hunkeler, Rationalizing the design of polymeric biomaterials. Trends in biotechnology, 1999. 17(10): p. 409-421.

30. Valencia, P.M., et al., Effects of ligands with different water solubilities on selfassembly and properties of targeted nanoparticles. Biomaterials, 2011. 32(26): p. 6226-6233.

31. Wu, L., et al., Long-acting injectable hormonal dosage forms for contraception. Pharmaceutical research, 2015. 32(7): p. 2180-2191.

32. Sitruk-Ware, R., A. Nath, and D.R. Mishell, Contraception technology: past, present and future. Contraception, 2013. 87(3): p. 319-330.

33. Darroch, J.E., G. Sedgh, and H. Ball, Contraceptive technologies: Responding to women's needs. New York: Guttmacher Institute, 2011. 201(1).

34. Halpern, V., et al., Towards the development of a longer-acting injectable contraceptive: past research and current trends. Contraception, 2015. 92(1): p. 39.

35. Schneider, M., et al., Crossing biological barriers for advanced drug delivery. European Journal of Pharmaceutics and Biopharmaceutics, 2013. 84(2): p. 239241.

36. Blanco, E., H. Shen, and M. Ferrari, Principles of nanoparticle design for overcoming biological barriers to drug delivery. Nature biotechnology, 2015. 33(9): p. 941-951.

37. Lockman, P.R., et al., Nanoparticle technology for drug delivery across the blood-brain barrier. Drug Development and Industrial Pharmacy, 2002. 28(1): p. 1-13. 
38. Gil, E.S., et al., Quaternary ammonium $\beta$-cyclodextrin nanoparticles for enhancing doxorubicin permeability across the in vitro blood-brain barrier. Biomacromolecules, 2009. 10(3): p. 505-516.

39. Bhaskar, S., et al., Multifunctional Nanocarriers for diagnostics, drug delivery and targeted treatment across blood-brain barrier: Perspectives on tracking and neuroimaging. Particle and Fibre Toxicology, 2010. 7.

40. Tian, B., et al., Novel surface-modified nanostructured lipid carriers with partially deacetylated water-soluble chitosan for efficient ocular delivery. Journal of Pharmaceutical Sciences, 2012. 101(3): p. 1040-1049.

41. Sultana, Y., et al., Nanotechnology in ocular delivery: Current and future directions. Drugs of Today, 2011. 47(6): p. 441-455.

42. Sharma, A., et al., Polyethylenimine-conjugated gold nanoparticles: Gene transfer potential and low toxicity in the cornea. Nanomedicine: Nanotechnology, Biology, and Medicine, 2011. 7(4): p. 505-513.

43. Luo, Q., et al., Nanostructured lipid carrier (NLC) coated with Chitosan Oligosaccharides and its potential use in ocular drug delivery system. Int J Pharm, 2011. 403(1-2): p. 185-191.

44. $\mathrm{Xu}, \mathrm{F}$., et al., Brain delivery and systemic effect of cationic albumin conjugated PLGA nanoparticles. Journal of drug targeting, 2009. 17(6): p. 423-434.

45. $\mathrm{Hu}, \mathrm{K}$., et al., Lactoferrin-conjugated PEG-PLA nanoparticles with improved brain delivery: in vitro and in vivo evaluations. Journal of controlled release, 2009. 134(1): p. 55-61.

46. Lu, W., et al., Cationic albumin conjugated pegylated nanoparticle with its transcytosis ability and little toxicity against blood-brain barrier. International journal of pharmaceutics, 2005. 295(1): p. 247-260.

47. Gao, X., et al., Lectin-conjugated PEG-PLA nanoparticles: preparation and brain delivery after intranasal administration. Biomaterials, 2006. 27(18): p. 3482-3490.

48. Xin, H., et al., Angiopep-conjugated poly (ethylene glycol)-co-poly ( $\varepsilon$ caprolactone) nanoparticles as dual-targeting drug delivery system for brain glioma. Biomaterials, 2011. 32(18): p. 4293-4305.

49. Xin, H., et al., Anti-glioblastoma efficacy and safety of paclitaxel-loading Angiopep-conjugated dual targeting PEG-PCL nanoparticles. Biomaterials, 2012. 33(32): p. 8167-8176.

50. Gil, E.S., et al., $\beta$-Cyclodextrin-poly ( $\beta$-amino ester) nanoparticles for sustained drug delivery across the blood-brain barrier. Biomacromolecules, 2012. 13(11): p. 3533-3541.

51. Qin, Y., et al., Liposome formulated with TAT-modified cholesterol for improving brain delivery and therapeutic efficacy on brain glioma in animals. International journal of pharmaceutics, 2011. 420(2): p. 304-312.

52. Krauze, M.T., et al., Real-time imaging and quantification of brain delivery of liposomes. Pharmaceutical research, 2006. 23(11): p. 2493-2504.

53. Gaillard, P.J., et al., Enhanced brain delivery of liposomal methylprednisolone improved therapeutic efficacy in a model of neuroinflammation. Journal of controlled release, 2012. 164(3): p. 364-369. 
54. Chen, H., et al., Lactoferrin-modified procationic liposomes as a novel drug carrier for brain delivery. European Journal of Pharmaceutical Sciences, 2010. 40(2): p. 94-102.

55. Puras, G., et al., Protamine/DNA/Niosome Ternary Nonviral Vectors for Gene Delivery to the Retina: The Role of Protamine. Molecular Pharmaceutics, 2015. 12(10): p. 3658-3671.

56. Puras, G., et al., A novel cationic niosome formulation for gene delivery to the retina. Journal of Controlled Release, 2014. 174: p. 27-36.

57. Blasi, P., et al., Solid lipid nanoparticles for targeted brain drug delivery. Advanced drug delivery reviews, 2007. 59(6): p. 454-477.

58. Yang, S.C., et al., Body distribution in mice of intravenously injected camptothecin solid lipid nanoparticles and targeting effect on brain. Journal of controlled release, 1999. 59(3): p. 299-307.

59. Huang, R., et al., The use of lactoferrin as a ligand for targeting the polyamidoamine-based gene delivery system to the brain. Biomaterials, 2008. 29(2): p. 238-246.

60. Oliveira, J.M., et al., Dendrimers and derivatives as a potential therapeutic tool in regenerative medicine strategies - a review. Progress in Polymer Science, 2010. 35(9): p. 1163-1194.

61. He, H., et al., PEGylated Poly (amidoamine) dendrimer-based dual-targeting carrier for treating brain tumors. Biomaterials, 2011. 32(2): p. 478-487.

62. Huang, S., et al., Dual targeting effect of Angiopep-2-modified, DNA-loaded nanoparticles for glioma. Biomaterials, 2011. 32(28): p. 6832-6838.

63. Yavuz, B., et al., In Vitro/In Vivo Evaluation of DexamethasonePAMAM Dendrimer Complexes for Retinal Drug Delivery. Journal of Pharmaceutical Sciences, 2015. 104(11): p. 3814-3823.

64. Kambhampati, S.P., et al., Systemic and Intravitreal Delivery of Dendrimers to Activated Microglia/Macrophage in Ischemia/Reperfusion Mouse Retina. Investigative Ophthalmology \& Visual Science, 2015. 56(8): p. 4413-4424.

65. Iezzi, R., et al., Dendrimer-based targeted intravitreal therapy for sustained attenuation of neuroinflammation in retinal degeneration. Biomaterials, 2012. 33(3): p. 979-988.

66. Marano, R.J., et al., Dendrimer delivery of an anti-VEGF oligonucleotide into the eye: a long-term study into inhibition of laser-induced CNV, distribution, uptake and toxicity. Gene Therapy, 2005. 12(21): p. 1544-1550.

67. Aktas, Y., et al., Development and brain delivery of chitosan-PEG nanoparticles functionalized with the monoclonal antibody OX26. Bioconjugate chemistry, 2005. 16(6): p. 1503-1511.

68. Trapani, A., et al., Characterization and evaluation of chitosan nanoparticles for dopamine brain delivery. International journal of pharmaceutics, 2011. 419(1): p. 296-307.

69. Md, S., et al., Bromocriptine loaded chitosan nanoparticles intended for direct nose to brain delivery: pharmacodynamic, pharmacokinetic and scintigraphy study in mice model. European Journal of Pharmaceutical Sciences, 2013. 48(3): p. 393-405. 
70. Raviña, M., et al., Hyaluronic acid/chitosan-g-poly (ethylene glycol) nanoparticles for gene therapy: an application for $p D N A$ and siRNA delivery. Pharmaceutical research, 2010. 27(12): p. 2544-2555.

71. Kreuter, J., et al., Direct evidence that polysorbate-80-coated poly (butylcyanoacrylate) nanoparticles deliver drugs to the CNS via specific mechanisms requiring prior binding of drug to the nanoparticles. Pharmaceutical research, 2003. 20(3): p. 409-416.

72. Kulkarni, P.V., et al., Quinoline-n-butylcyanoacrylate-based nanoparticles for brain targeting for the diagnosis of Alzheimer's disease. Wiley Interdisciplinary Reviews: Nanomedicine and Nanobiotechnology, 2010. 2(1): p. 35-47.

73. Alyautdin, R.N., et al., Delivery of loperamide across the blood-brain barrier with polysorbate 80-coated polybutylcyanoacrylate nanoparticles. Pharmaceutical research, 1997. 14(3): p. 325-328.

74. Gulyaev, A.E., et al., Significant transport of doxorubicin into the brain with polysorbate 80-coated nanoparticles. Pharmaceutical research, 1999. 16(10): $\mathrm{p}$. 1564-1569.

75. Ulbrich, K., et al., Transferrin-and transferrin-receptor-antibody-modified nanoparticles enable drug delivery across the blood-brain barrier (BBB). European Journal of Pharmaceutics and Biopharmaceutics, 2009. 71(2): p. 251256.

76. Dadparvar, M., et al., HI 6 human serum albumin nanoparticles-Development and transport over an in vitro blood-brain barrier model. Toxicology letters, 2011. 206(1): p. 60-66.

77. Zensi, A., et al., Albumin nanoparticles targeted with Apo E enter the CNS by transcytosis and are delivered to neurones. Journal of Controlled Release, 2009. 137(1): p. 78-86.

78. Prades, R., et al., Delivery of gold nanoparticles to the brain by conjugation with a peptide that recognizes the transferrin receptor. Biomaterials, 2012. 33(29): $\mathrm{p}$. 7194-7205.

79. Samad, A., Y. Sultana, and M. Aqil, Liposomal drug delivery systems: an update review. Current drug delivery, 2007. 4(4): p. 297-305.

80. Janaszewska, A., et al., The biodistribution of maltotriose modified poly (propylene imine)(PPI) dendrimers conjugated with fluorescein-proofs of crossing blood-brain-barrier. New Journal of Chemistry, 2012. 36(2): p. 350353.

81. De, S. and D. Robinson, Polymer relationships during preparation of chitosanalginate and poly-l-lysine-alginate nanospheres. Journal of Controlled Release, 2003. 89(1): p. 101-112.

82. Gref, R., et al., Biodegradable long-circulating polymeric nanospheres. Science, 1994. 263(5153): p. 1600-1603.

83. José Alonso, M., Nanomedicines for overcoming biological barriers. Biomedicine \&amp; Pharmacotherapy, 2004. 58(3): p. 168-172.

84. Jung, T., et al., Biodegradable nanoparticles for oral delivery of peptides: is there a role for polymers to affect mucosal uptake? European Journal of Pharmaceutics and Biopharmaceutics, 2000. 50(1): p. 147-160. 
85. Peer, D., et al., Nanocarriers as an emerging platform for cancer therapy. Nature nanotechnology, 2007. 2(12): p. 751-760.

86. Soppimath, K.S., et al., Biodegradable polymeric nanoparticles as drug delivery devices. Journal of Controlled Release, 2001. 70(1-2): p. 1-20.

87. Gil, E.S., et al., Quaternary ammonium $\beta$-cyclodextrin nanoparticles for enhancing doxorubicin permeability across the in vitro blood-brain barrier. Biomacromolecules, 2009. 10(3): p. 505-516.

88. Hervé, F., N. Ghinea, and J.-M. Scherrmann, CNS delivery via adsorptive transcytosis. The AAPS journal, 2008. 10(3): p. 455-472.

89. Petitti, D.B., et al., Stroke in users of low-dose oral contraceptives. New England journal of medicine, 1996. 335(1): p. 8-15.

90. Sagraves, R., Estrogen therapy for postmenopausal symptoms and prevention of osteoporosis. The Journal of Clinical Pharmacology, 1995. 35(9S): p. 2S-10S.

91. Dupont, A., et al., Comparative endocrinological and clinical effects of percutaneous estradiol and oral conjugated estrogens as replacement therapy in menopausal women. Maturitas, 1991. 13(4): p. 297-311.

92. Trussell, J., Contraceptive failure in the United States. Contraception, 2011. 83(5): p. 397-404.

93. Medicine, P.C.o.t.A.S.f.R., Hormonal contraception: recent advances and controversies. Fertility and sterility, 2004. 82(2): p. 520-526.

94. d'Arcangues, C. and R.C. Snow, Injectable contraceptives, in Fertility ControlUpdate and Trends. 1999, Springer. p. 121-149.

95. Chue, P., Risperidone long-acting injection. Expert Review of Neurotherapeutics, 2003. 3(4): p. 435-446.

96. Hatefi, A. and B. Amsden, Biodegradable injectable in situ forming drug delivery systems. Journal of Controlled Release, 2002. 80(1-3): p. 9-28.

97. Packhaeuser, C.B., et al., In situ forming parenteral drug delivery systems: An overview. European Journal of Pharmaceutics and Biopharmaceutics, 2004. 58(2): p. 445-455.

98. Garza-Flores, J., P.E. Hall, and G. Perez-Palacios, Long-acting hormonal contraceptives for women. Journal of Steroid Biochemistry and Molecular Biology, 1991. 40(4-6): p. 697-704.

99. Beck, L.R., et al., Long-acting injectable microsphere formulation for the parenteral administration of levonorgestrel. Advances in Contraception, 1985. 1(2): p. 119-129.

100. Wang, S.H., et al., Controlled release of levonorgestrel from biodegradable poly(D,L-lactide-co-glycolide) microspheres: In vitro and in vivo studies. International Journal of Pharmaceutics, 2005. 301(1-2): p. 217-225.

101. Dhanaraju, M.D., et al., Biodegradation and biocompatibility of contraceptivesteroid-loaded poly (dl-lactide-co-glycolide) injectable microspheres: in vitro and in vivo study. Contraception, 2006. 74(2): p. 148-156.

102. Sun, Y., et al., Synchronic release of two hormonal contraceptives for about one month from the PLGA microspheres: in vitro and in vivo studies. J Control Release, 2008. 129(3): p. 192-9. 
103. Machado, S.R., et al., Preparation and characterization of D, L-PLA loaded 17beta-Estradiol valerate by emulsion/evaporation methods. J Microencapsul, 2009. 26(3): p. 202-13.

104. Puthli, S. and P. Vavia, Formulation and performance characterization of radiosterilized "progestin-only" microparticles intended for contraception. AAPS PharmSciTech, 2009. 10(2): p. 443-52.

105. Trantolo, D., et al., Biodegradable systems for long-acting nesterone, in Handbook of pharmaceutical controlled release technology, D. Wise, Editor. 2000, Marcel Dekker, Inc.: New York.

106. Beck, L.R., V.Z. Pope, and C.E. Flowers Jr, Poly(DL-lactide-coglycolide)/norethisteron microcapsules: An injectable biodegradable contraceptive. Biology of Reproduction, 1983. 28(1): p. 186-195.

107. Rivera, R., et al., Norethisterone contraceptive microspheres. Journal of Steroid Biochemistry, 1987. 27(4-6): p. 1003-1007.

108. Grubb, G.S., et al., A comparative evaluation of the safety and contraceptive effectiveness of $65 \mathrm{mg}$ and $100 \mathrm{mg}$ of 90-day norethindrone (NET) injectable microspheres: A multicenter study. Fertility and Sterility, 1989. 51(5): p. 803-810.

109. Singh, M., et al., Contraceptive efficacy of norethindrone encapsulated in injectable biodegradable polydl-lactide-co-glycolide microspheres (NET-90): Phase III clinical study. Advances in Contraception, 1997. 13(1): p. 1-11.

110. Kempe, S. and K. Mäder, In situ forming implants - An attractive formulation principle for parenteral depot formulations. Journal of Controlled Release, 2012. 161(2): p. 668-679.

111. Junkmann, K., Long-acting steroids in reproduction. Recent Progress in Hormone Research, 1957. 13: p. 389-419.

112. Shearman, R.P., The development of depot contraceptives. Journal of Steroid Biochemistry, 1975. 6(6): p. 899-902.

113. Peralta, O., Injectable hormonal contraceptives: an overview. Gynaecology Forum, 2000. 5(1): p. http://www.medforum.nl/gynfo/leading_article1.asp.

114. Eunice Kennedy Shriver National Institute of Child Health and Human Development (NIHCD), A Phase I Study to Evaluate the Pharmacokinetic and Pharmacodynamic Profile of a Single Injection of Levonorgestel Butanoate for Female Contraception. In: ClinicalTrials.gov [Internet]. Bethesda (MD): National Library of Medicine (US), 2014 May 12 [cited 2014 Sep 7].(Available from: http://clinicaltrials.gov/show/NCT02173808 NLM Identifier: NCT02173808.).

115. High-priority leads for development of new methods of fertility regulation. https://apps.who.int/hrp/progress/44/02.html 1997.

116. Conrad. http://www.conrad.org/contraception-grid.html. 2012.

117. Kumari, A., S.K. Yadav, and S.C. Yadav, Biodegradable polymeric nanoparticles based drug delivery systems. Colloids and Surfaces B: Biointerfaces, 2010. 75(1): p. 1-18.

118. Tracy, M., et al., Factors affecting the degradation rate of poly (lactide-coglycolide) microspheres in vivo and in vitro. Biomaterials, 1999. 20(11): p. 10571062. 
119. Yang, L. and P. Alexandridis, Physicochemical aspects of drug delivery and release from polymer-based colloids. Current opinion in colloid \& interface science, 2000. 5(1): p. 132-143.

120. Gander, B., et al., Thermodynamic approach to protein microencapsulation into poly (D, L-lactide) by spray drying. International Journal of Pharmaceutics, 1996. 129(1): p. 51-61.

121. Blanco-Príeto, M.a.J., et al., Importance of single or blended polymer types for controlled in vitro release and plasma levels of a somatostatin analogue entrapped in PLA/PLGA microspheres. Journal of controlled release, 2004. 96(3): p. 437-448.

122. Göpferich, A., Polymer bulk erosion. Macromolecules, 1997. 30(9): p. 25982604.

123. Vert, M., Aliphatic polyesters: great degradable polymers that cannot do everything. Biomacromolecules, 2005. 6(2): p. 538-546.

124. Doi, Y. and A. Steinbüchel, Biopolymers, polyesters III-applications and commercial products. Vol. 4. 2002: Wiley-Blackwell.

125. Edlund, U., et al., Degradable aliphatic polyesters. Vol. 157. 2003: Springer.

126. Göpferich, A., Mechanisms of polymer degradation and erosion. Biomaterials, 1996. 17(2): p. 103-114.

127. Albertsson, A.-C. and I.K. Varma, Aliphatic polyesters: synthesis, properties and applications, in Degradable Aliphatic Polyesters. 2002, Springer. p. 1-40.

128. Chen, S., et al., In vivo absorption of steroidal hormones from smart polymer based delivery systems. Journal of pharmaceutical sciences, 2010. 99(8): p. 33813388.

129. Okada, H., One- and three-month release injectable microspheres of the LH-RH superagonist leuprorelin acetate. Advanced Drug Delivery Reviews, 1997. 28(1): p. 43-70.

130. Sharifi, R., et al., Therapeutic effects of leuprorelin microspheres in prostate cancer. Advanced Drug Delivery Reviews, 1997. 28(1): p. 121-138.

131. Putney, S.D. and P.A. Burke, Improving protein therapeutics with sustainedrelease formulations. Nature Biotechnology, 1998. 16(2): p. 153.

132. Freiberg, S. and X.X. Zhu, Polymer microspheres for controlled drug release. International Journal of Pharmaceutics, 2004. 282(1-2): p. 1-18.

133. Okada, H., et al., Preparation of three-month depot injectable microspheres of leuprorelin acetate using biodegradable polymers. Pharmaceutical Research, 1994. 11(8): p. 1143-1147.

134. Clarck, S.L., et al., Long-term delivery of ivermectin by use of poly(D,L-lactic-coglycolic)acid microparticles in dogs. American Journal of Veterinary Research, 2004. 65(6): p. 752-757.

135. Beck, L.R., D.R. Cowsar, and D.H. Lewis, New long-acting injectable microcapsule contraceptive system. American Journal of Obstetrics and Gynecology, 1979. 135(3): p. 419-426.

136. Tice, T.R. and R.M. Gilley, Preparation of injectable controlled-release microcapsules by a solvent-evaporation process. Journal of Controlled Release, 1985. 2: p. 343-352. 
137. Cowsar, D.R., et al., Poly(lactide-co-glycolide) microcapsules for controlled release of steroids. Methods in Enzymology, 1985. 112: p. 101-116.

138. Dhanaraju, M.D., R. Jayakumar, and C. Vamsadhara, Influence of manufacturing parameters on development of contraceptive steroid loaded injectable microspheres. Chemical and pharmaceutical bulletin, 2004. 52(8): p. 976-979.

139. Song, C.X., H.F. Sun, and X.D. Feng, Microspheres of biodegradable block copolymer for long-acting controlled delivery of contraceptives. Polymer Journal, 1987. 19(5): p. 485-491.

140. Gu, Z.W., et al., Biodegradable block copolymer matrices for long-acting contraceptives with constant release. Journal of Controlled Release, 1992. 22(1): p. 3-14.

141. Buntner, B., et al., The application of microspheres from the copolymers of lactide and $\varepsilon$-caprolactone to the controlled release of steroids. Journal of Controlled Release, 1998. 56(1-3): p. 159-167.

142. Dhanaraju, M.D., et al., Preparation and characterization of injectable microspheres of contraceptive hormones. International journal of pharmaceutics, 2003. 268(1): p. 23-29.

143. Dhanaraju, M.D., et al., Characterization of polymeric poly( $\varepsilon$-caprolactone) injectable implant delivery system for the controlled delivery of contraceptive steroids. Journal of Biomedical Materials Research - Part A, 2006. 76(1): p. 6372.

144. Berkland, C., K. Kim, and D.W. Pack, PLG microsphere size controls drug release rate through several competing factors. Pharmaceutical Research, 2003. 20(7): p. 1055-1062.

145. Duncanson, W.J., et al., Microfluidic synthesis of advanced microparticles for encapsulation and controlled release. Lab on a Chip - Miniaturisation for Chemistry and Biology, 2012. 12(12): p. 2135-2145.

146. Berkland, C., et al., Precise control of PLG microsphere size provides enhanced control of drug release rate. Journal of Controlled Release, 2002. 82(1): p. 137147.

147. Zhong-wei, G., et al., Biodegradable block copolymer matrices for long-acting contraceptives with constant release. Journal of controlled release, 1992. 22(1): p. 3-14.

148. Schindler, A., et al., Biodegradable polymers for sustained drug delivery, in Contemporary topics in polymer science. 1977, Springer. p. 251-289.

149. Pitt, C.G., et al., Sustained drug delivery systems. I. The permeability of poly ( $\epsilon-$ caprolactone), poly (DL-lactic acid), and their copolymers. Journal of biomedical materials research, 1979. 13(3): p. 497-507.

150. Li, Y.X. and X.D. Feng. Biodegradable polymeric matrix for long-acting and zero-order release drug delivery systems. in Makromolekulare Chemie. Macromolecular Symposia. 1990. Wiley Online Library.

151. Beck, L.R. and D. Cowsar, Biodegradable microsphere contraceptive system. Acta Europaea Fertilitatis, 1980. 11(2): p. 139-150.

152. Beck, L.R. and V.Z. Pope, Long-acting injectable norethisterone contraceptive system: review of clinical studies. Research frontiers in fertility regulation : RFFR / PARFR, 1984. 3(2): p. 1-10. 
153. Huang, X. and C.S. Brazel, On the importance and mechanisms of burst release in matrix-controlled drug delivery systems. Journal of Controlled Release, 2001. 73(2-3): p. 121-136.

154. Huang, X., B.L. Chestang, and C.S. Brazel, Minimization of initial burst in poly(vinyl alcohol) hydrogels by surface extraction and surface-preferential crosslinking. International Journal of Pharmaceutics, 2002. 248(1-2): p. 183-192.

155. Wu, L. and C.S. Brazel, Modifying the release of proxyphylline from PVA hydrogels using surface crosslinking. International Journal of Pharmaceutics, 2008. 349(1-2): p. 144-151.

156. Lee, S.H., et al., Core-shell poly(D,L-lactide-co-glycolide)/poly(ethyl 2cyanoacrylate) microparticles with doxorubicin to reduce Initial burst release. Macromolecular Research, 2009. 17(12): p. 1010-1014.

157. $\mathrm{Wu}$, J., et al., Fabrication and characterization of monodisperse PLGA-alginate core-shell microspheres with monodisperse size and homogeneous shells for controlled drug release. Acta Biomaterialia, 2013. 9(7): p. 7410-7419.

158. Cong, H. and L.R. Beck, Preparation and pharmacokinetic evaluation of a modified long-acting injectable norethisterone microsphere. Advances in Contraception, 1991. 7(2-3): p. 251-256.

159. Jameela, S.R., et al., Progesterone-loaded chitosan microspheres: A long acting biodegradable controlled delivery system. Journal of Controlled Release, 1998. 52(1-2): p. 17-24.

160. Latha, M.S., et al., Progesterone release from glutaraldehyde cross-linked casein microspheres: In vitro studies and in vivo response in rabbits. Contraception, 2000. 61(5): p. 329-334.

161. Lee, T.K., T.D. Sokoloski, and G.P. Royer, Serum albumin beads: an injectable, biodegradable system for the sustained release of drugs. Science, 1981. 213(4504): p. 233-235.

162. Lei, Y. and B. Lu, Studies on preparation technology of microspheres of contraceptives. Acta Pharmaceutica Sinica, 1993. 28(4): p. 296-301.

163. Lu, B., R.L. Guo, and C. Liu, Studies on an injection of microencapsulated levonorgestrel, in Microencapsulation of Drugs, T.L. Whateley, Editor. 1992, Harwood academic publishers: UK. p. 103-121.

164. Lu, B., Z. Wang, and H. Yang, Long-acting delivery microspheres of levonorgestrolpoly (3-hydroxybutyrate): their preparation, characterization and contraceptive tests on mice. Journal of microencapsulation, 2001. 18(1): p. 55-64.

165. Beck, L.R., R.A. Ramos, and C.E. Flowers Jr, Clinical evaluation of injectable biodegradable contraceptive system. American Journal of Obstetrics and Gynecology, 1981. 140(7): p. 799-806.

166. Berkland, C., et al., Three-month, zero-order piroxicam release from monodispersed double-walled microspheres of controlled shell thickness. Journal of Biomedical Materials Research - Part A, 2004. 70(4): p. 576-584.

167. Pollauf, E.J., K.K. Kim, and D.W. Pack, Small-molecule release from poly(D,Llactide)/poly(D,L-lactide-co-glycolide) composite microparticles. Journal of Pharmaceutical Sciences, 2005. 94(9): p. 2013-2022. 
168. Dunn, R., et al., Sustained release of cisplatin in dogs from an injectable implant delivery system. Journal of bioactive and compatible polymers, 1996. 11(4): p. 286-300.

169. Eliaz, R.E., D. Wallach, and J. Kost, Delivery of soluble tumor necrosis factor receptor from in-situ forming PLGA implants: in-vivo. Pharmaceutical research, 2000. 17(12): p. 1546-1550.

170. Kempe, S. and K. Mäder, In situ forming implants - an attractive formulation principle for parenteral depot formulations. Journal of controlled release, 2012. 161(2): p. 668-679.

171. Hatefi, A. and B. Amsden, Biodegradable injectable in situ forming drug delivery systems. Journal of Controlled Release, 2002. 80(1): p. 9-28.

172. Bedi, S., A novel shear thinning and thixotropic PLGA micro particulate suspension system for controlled drug delivery. 2011: THE UNIVERSITY OF TENNESSEE HEALTH SCIENCE CENTER.

173. Wu, H., et al., Design and evaluation of baicalin-containing in situ pH-triggered gelling system for sustained ophthalmic drug delivery. International Journal of Pharmaceutics, 2011.410(1-2): p. 31-40.

174. Başaran, B. and A. Bozkir, Thermosensitive and pH induced in situ ophthalmic gelling system for ciprofloxacin hydrochloride: Hydroxypropyl- $\beta$-cyclodextrin complex. Acta Poloniae Pharmaceutica - Drug Research, 2012. 69(6): p. 11371147.

175. Gupta, S.K. and I.J. Singhvi, Sustained ophthalmic delivery of moxifloxacin hydrochloride from an $\mathrm{pH}$ triggered in situ gelling system. Research Journal of Pharmacy and Technology, 2012. 5(12): p. 1538-1542.

176. Royals, M.A., et al., Biocompatibility of a biodegradable in situ forming implant system in rhesus monkeys. Journal of Biomedical Materials Research, 1999. 45(3): p. 231-239.

177. Astaneh, R., et al., Effects of ethyl benzoate on performance, morphology, and erosion of PLGA implants formed in situ. Advances in Polymer Technology, 2008. 27(1): p. 17-26.

178. Nair, L.S., et al., Development of injectable thermogelling chitosan-inorganic phosphate solutions for biomedical applications. Biomacromolecules, 2007. 8(12): p. 3779-3785.

179. Pandya, T.P., M.K. Modasiya, and V.M. Patel, Sustained ophthalmic delivery of ciprofloxacin hydrochloride from an ion-activated in situ gelling system. Der Pharmacia Lettre, 2011. 3(3): p. 404-410.

180. Couffin-Hoarau, A.-C., et al., In situ-forming pharmaceutical organogels based on the self-assembly of L-alanine derivatives. Pharmaceutical research, 2004. 21(3): p. 454-457.

181. Thakur, R.R.S., H.L. McMillan, and D.S. Jones, Solvent induced phase inversionbased in situ forming controlled release drug delivery implants. Journal of Controlled Release, 2014. 176: p. 8-23.

182. Dunn, R.L., et al., Biodegradable in-situ forming implants and methods of producing the same. 1990, Google Patents.

183. Dunn, R.L., et al., Biodegradable in-situ forming implants and methods of producing the same. 1998, Google Patents. 
184. Graham, P., K. Brodbeck, and A. McHugh, Phase inversion dynamics of PLGA solutions related to drug delivery. Journal of Controlled Release, 1999. 58(2): p. 233-245.

185. Patel, R.B., et al., Effect of injection site on in situ implant formation and drug release in vivo. Journal of Controlled Release, 2010. 147(3): p. 350-358.

186. Körber, M. and R. Bodmeier, Development of an in situ forming PLGA drug delivery system: I. Characterization of a non-aqueous protein precipitation. european journal of pharmaceutical sciences, 2008. 35(4): p. 283-292.

187. Packhaeuser, C., et al., In situ forming parenteral drug delivery systems: an overview. European Journal of Pharmaceutics and Biopharmaceutics, 2004. 58(2): p. 445-455.

188. Ruel-Gariepy, E. and J.-C. Leroux, In situ-forming hydrogels-review of temperature-sensitive systems. European Journal of Pharmaceutics and Biopharmaceutics, 2004. 58(2): p. 409-426.

189. Kranz, H. and R. Bodmeier, Structure formation and characterization of injectable drug loaded biodegradable devices: in situ implants versus in situ microparticles. European journal of pharmaceutical sciences, 2008. 34(2): p. 164172.

190. Chitkara, D., et al., Biodegradable injectable in situ depot-forming drug delivery systems. Macromolecular bioscience, 2006. 6(12): p. 977-990.

191. Wang, L., S. Venkatraman, and L. Kleiner, Drug release from injectable depots: two different in vitro mechanisms. Journal of controlled release, 2004. 99(2): p. 207-216.

192. Brodbeck, K.J., S. Pushpala, and A.J. McHugh, Sustained release of human growth hormone from PLGA solution depots. Pharmaceutical research, 1999. 16(12): p. 1825-1829.

193. Patel, R.B., et al., Characterization of formulation parameters affecting low molecular weight drug release from in situ forming drug delivery systems. Journal of Biomedical Materials Research Part A, 2010. 94(2): p. 476-484.

194. Parent, M., et al., PLGA in situ implants formed by phase inversion: Critical physicochemical parameters to modulate drug release. Journal of Controlled Release, 2013. 172(1): p. 292-304.

195. Gao, Z.-h., et al., Controlled release of contraceptive steroids from biodegradable and injectable gel formulations: in vivo evaluation. Pharmaceutical research, 1995. 12(6): p. 864-868.

196. Gao, Z.-H., et al., Controlled release of a contraceptive steroid from biodegradable and injectable gel formulations: in vitro evaluation. Pharmaceutical research, 1995. 12(6): p. 857-863.

197. Wheaton, C., et al., The use of long acting subcutaneous levonorgestrel (LNG) gel depot as an effective contraceptive option for cotton-top tamarins (Saguinus oedipus). Zoo biology, 2011. 30(5): p. 498-522.

198. Gao, Z., et al., Controlled release of a contraceptive steroid from biodegradable and injectable gel formulations: In vitro evaluation. Pharmaceutical Research, 1995. 12(6): p. 857-863. 
199. Thosar, S.S., Controlled release of a contraceptive steroid from biodegradable and injectable formulations: In vitro and in vivo evaluations. The University of Tennessee Health Science Center, Thesis, 1997.

200. Thosar, S.S., et al., Evaluation of the Effects of Varying Formulation Factors on the In Vitro Release of Levonorgestrel from a Biodegradable Injectable Drug Delivery System. . Pharmaceutical Research, 1996. 13 p. S-298.

201. Camargo, J., et al., Injectable PLA-based in situ forming implants for controlled release of Ivermectin a BCS Class II drug: solvent selection based on physicochemical characterization. Drug development and industrial pharmacy, 2013. 39(1): p. 146-155.

202. Rowe, R.C., P.J. Sheskey, and M. Quinn, Handbook of pharmaceutical excipients-7th edition. Pharm. Dev. Technol., 2013. 18: p. 544.

203. Riddick, J., W. Bunger, and T. Sakano, Techniques of Chemistry 4th ed., Volume II. Organic Solvents. 1985, New York, NY: John Wiley and Sons.

204. Jacko, M. and S. Lee, Kirk-Othmer encyclopedia of chemical technology. 1992.

205. Altshuller, A. and H. Everson, The solubility of ethyl acetate in water. Journal of the American Chemical Society, 1953. 75(7): p. 1727-1727.

206. Tell, L., et al., A comparison of the effects of slow release, injectable levonorgestrel and depot medroxyprogesterone acetate on egg production in Japanese quail (Coturnix coturnix japonica). Journal of Avian Medicine and Surgery, 1999. 13(1): p. 23-31.

207. Looper, S., et al., Efficacy of levonorgestrel when administered as an irradiated, slow-release injectable matrix for feline contraception. Zoo Biology, 2001. 20(5): p. 407-421.

208. Wheaton, C.J., et al., The use of long acting subcutaneous levonorgestrel (LNG) gel depot as an effective contraceptive option for -top tamarins (Saguinus oedipus). Zoo Biology, 2011. 30(5): p. 498-522.

209. Yewey, G.L.K., Nancy L.; Dunn, Richard L.; Radomsky, Michael L.; Brouwer, Gerbrand; Tipton, Arthur J., Liquid delivery compositions, in US Patent 5780044 , U. Patent, Editor. 1998: USA.

210. Chen, S. and J. Singh, In vitro release of levonorgestrel from phase sensitive and thermosensitive smart polymer delivery systems. Pharmaceutical Development and Technology, 2005. 10(2): p. 319-325.

211. Brodbeck, K.J.G.-D., Ann T.; Shen, Theodore Tao-Ian, Gel composition and methods, in US Patent 6130200, U. patent, Editor. 2000: USA.

212. Lalitkumar, P.G., C. Berger, and K. Gemzell-Danielsson, Emergency contraception. Best Pract Res Clin Endocrinol Metab, 2013. 27(1): p. 91-101.

213. Raymond, E.G. and K. Cleland, Clinical practice. Emergency contraception. N Engl J Med, 2015. 372(14): p. 1342-8.

214. Koyama, A., L. Hagopian, and J. Linden, Emerging options for emergency contraception. Clin Med Insights Reprod Health, 2013. 7: p. 23-35.

215. Beck, L.R., et al., Clinical evaluation of injectable biodegradable contraceptive system. American journal of obstetrics and gynecology, 1981. 140(7): p. 799-806.

216. Buntner, B., et al., The application of microspheres from the copolymers of lactide and $\epsilon$-caprolactone to the controlled release of steroids. Journal of controlled release, 1998. 56(1): p. 159-167. 
217. Wu, L., et al., Long-Acting Injectable Hormonal Dosage Forms for Contraception. Pharmaceutical Research, 2015.

218. Goldman, J.M., A.S. Murr, and R.L. Cooper, The rodent estrous cycle: characterization of vaginal cytology and its utility in toxicological studies. Birth Defects Research Part B: Developmental and Reproductive Toxicology, 2007. 80(2): p. 84-97.

219. Freiberg, S. and X. Zhu, Polymer microspheres for controlled drug release. International journal of pharmaceutics, 2004. 282(1): p. 1-18.

220. Brodbeck, K., J. DesNoyer, and A. McHugh, Phase inversion dynamics of PLGA solutions related to drug delivery: Part II. The role of solution thermodynamics and bath-side mass transfer. Journal of Controlled Release, 1999. 62(3): p. 333344.

221. Messaritaki, A., et al., NMR and confocal microscopy studies of the mechanisms of burst drug release from PLGA microspheres. Journal of controlled release, 2005. 108(2): p. 271-281.

222. Licea-Perez, H., et al., A semi-automated 96-well plate method for the simultaneous determination of oral contraceptives concentrations in human plasma using ultra performance liquid chromatography coupled with tandem mass spectrometry. J Chromatogr B Analyt Technol Biomed Life Sci, 2007. 852(1-2): p. 69-76.

223. Moser, C., et al., Simultaneous online SPE-LC-MS/MS quantification of six widely used synthetic progestins in human plasma. Anal Bioanal Chem, 2012. 403(4): p. 961-72.

224. Theron, H.B., et al., Selective and sensitive liquid chromatography-tandem mass spectrometry method for the determination of levonorgestrel in human plasma. J Chromatogr B Analyt Technol Biomed Life Sci, 2004. 813(1-2): p. 331-6.

225. Zhao, L.Z., et al., Determination of levonorgestrel in human plasma by liquid chromatography-tandem mass spectrometry method: application to a bioequivalence study of two formulations in healthy volunteers. Biomed Chromatogr, 2008. 22(5): p. 519-26.

226. Al-Odaini, N.A., et al., Multi-residue analytical method for human pharmaceuticals and synthetic hormones in river water and sewage effluents by solid-phase extraction and liquid chromatography-tandem mass spectrometry. $\mathrm{J}$ Chromatogr A, 2010. 1217(44): p. 6791-806.

227. Viglino, L., et al., Analysis of natural and synthetic estrogenic endocrine disruptors in environmental waters using online preconcentration coupled with LC-APPI-MS/MS. Talanta, 2008. 76(5): p. 1088-96.

228. Liu, Z.H., et al., Occurrence, fate and removal of synthetic oral contraceptives (SOCs) in the natural environment: a review. Sci Total Environ, 2011. 409(24): p. 5149-61.

229. Lopez de Alda, M.J. and D. Barcelo, Determination of steroid sex hormones and related synthetic compounds considered as endocrine disrupters in water by liquid chromatography-diode array detection-mass spectrometry. J Chromatogr A, 2000. 892(1-2): p. 391-406. 
230. Ananthula, S., et al., Development and validation of sensitive LC/MS/MS method for quantitative bioanalysis of levonorgestrel in rat plasma and application to pharmacokinetics study. Journal of Chromatography B, 2015. 1003: p. 47-53.

231. Naqvi, R.H., et al., Pharmacokinetics of levonorgestrel in the rat. Contraception, 1984. 30(1): p. 81-88.

232. Ma, G., et al., A biodegradable levonorgestrel-releasing implant made of PCL/F68 compound as tested in rats and dogs. Contraception, 2006. 74(2): p. 141-147.

233. Vickery, Z., et al., Weight change at 12 months in users of three progestin-only contraceptive methods. Contraception, 2013. 88(4): p. 503-508.

234. Monies, G. and E. Luque, Effects of ovarian steroids on vaginal smears in the rat. Cells Tissues Organs, 1988. 133(3): p. 192-199.

235. McHugh, A., The role of polymer membrane formation in sustained release drug delivery systems. Journal of controlled release, 2005. 109(1): p. 211-221.

236. Astaneh, R., et al., Changes in morphology of in situ forming PLGA implant prepared by different polymer molecular weight and its effect on release behavior. Journal of pharmaceutical sciences, 2009. 98(1): p. 135-145.

237. Liu, Q., et al., In vitro and in vivo study of thymosin alphal biodegradable in situ forming poly (lactide-co-glycolide) implants. International journal of pharmaceutics, 2010. 397(1): p. 122-129.

238. Solorio, L., et al., Noninvasive characterization of in situ forming implants using diagnostic ultrasound. Journal of Controlled Release, 2010. 143(2): p. 183-190.

239. Zhang, Q., Development of a Novel Vaccine Adjuvant System Utilizing an in Situ Implant System to Modified Release. 2014, The University of Tennessee.

240. Janagam, D.R., et al., An accelerated release study to evaluate long-acting contraceptive levonorgestrel-containing in situ forming depot systems. Pharmaceutics, 2016. 8(3): p. 28.

241. Luan, X. and R. Bodmeier, In situ forming microparticle system for controlled delivery of leuprolide acetate: influence of the formulation and processing parameters. European journal of pharmaceutical sciences, 2006. 27(2): p. 143149.

242. Moens, E. and M. Veldhoen, Epithelial barrier biology: good fences make good neighbours. Immunology, 2012. 135(1): p. 1-8.

243. Barar, J., A.R. Javadzadeh, and Y. Omidi, Ocular novel drug delivery: impacts of membranes and barriers. Expert opinion on drug delivery, 2008. 5(5): p. 567-581.

244. Rubin, L. and J. Staddon, The cell biology of the blood-brain barrier. Annual review of neuroscience, 1999. 22(1): p. 11-28.

245. Misra, A., et al., Drug delivery to the central nervous system: a review. J Pharm Pharm Sci, 2003. 6(2): p. 252-273.

246. Urtti, A., Challenges and obstacles of ocular pharmacokinetics and drug delivery. Advanced drug delivery reviews, 2006. 58(11): p. 1131-1135.

247. Hamman, J.H., G.M. Enslin, and A.F. Kotzé, Oral delivery of peptide drugs. BioDrugs, 2005. 19(3): p. 165-177.

248. Anderson, J.M. and C.M. Van Itallie, Physiology and function of the tight junction. Cold Spring Harbor perspectives in biology, 2009. 1(2): p. a002584. 
249. Kievit, F.M. and M. Zhang, Cancer nanotheranostics: improving imaging and therapy by targeted delivery across biological barriers. Advanced materials, 2011. 23(36).

250. Gaudana, R., et al., Ocular drug delivery. The AAPS journal, 2010. 12(3): p. 348360.

251. Ghate, D. and H.F. Edelhauser, Ocular drug delivery. Expert Opinion on Drug Delivery, 2006. 3(2): p. 275-287.

252. Lee, V.H.L. and J.R. Robinson, Review: Topical ocular drug delivery: Recent developments and future challenges. Journal of Ocular Pharmacology, 1986. 2(1): p. 67-108.

253. Worakul, N. and J.R. Robinson, Ocular pharmacokinetics/pharmacodynamics. European journal of pharmaceutics and biopharmaceutics, 1997. 44(1): p. 71-83.

254. Klyce, S.D. and C.E. Crosson, Transport processes across the rabbit corneal epithelium: a review. Current eye research, 1985. 4(4): p. 323-331.

255. Ahmed, I. and T.F. Patton, Importance of the noncorneal absorption route in topical ophthalmic drug delivery. Investigative Ophthalmology and Visual Science, 1985. 26(4): p. 584-587.

256. Watsky, M.A., M.M. Jablonski, and H.F. Edelhauser, Comparison of conjunctival and corneal surface areas in rabbit and human. Current Eye Research, 1988. 7(5): p. 483-486.

257. Sasaki, H., et al., Different effects of absorption promoters on corneal and conjunctival penetration of ophthalmic beta-blockers. Pharmaceutical Research, 1995. 12(8): p. 1146-1150.

258. Ahmed, I., et al., Physicochemical determinants of drug diffusion across the conjunctiva, sclera, and cornea. Journal of Pharmaceutical Sciences, 1987. 76(8): p. 583-586.

259. She, S.C., L.P. Steahly, and E.J. Moticka, Intracameral injection of allogeneic lymphocytes enhances corneal graft survival. Investigative Ophthalmology and Visual Science, 1990. 31(10): p. 1950-1956.

260. Lane, S.S., et al., Evaluation of the safety of prophylactic intracameral moxifloxacin in cataract surgery. Journal of Cataract and Refractive Surgery, 2008. 34(9): p. 1451-1459.

261. Braga-Mele, R., et al., Intracameral antibiotics: Safety, efficacy, and preparation. Journal of Cataract and Refractive Surgery, 2014. 40(12): p. 2134-2142.

262. Vazirani, J. and S. Basu, Role of topical, subconjunctival, intracameral, and irrigative antibiotics in cataract surgery. Current Opinion in Ophthalmology, 2013. 24(1): p. 60-65.

263. Ahmed, I., et al., Physicochemical determinants of drug diffusion across the conjunctiva, sclera, and cornea. Journal of pharmaceutical sciences, 1987. 76(8): p. 583-586.

264. Prausnitz, M.R. and J.S. Noonan, Permeability of cornea, sclera, and conjunctiva: a literature analysis for drug delivery to the eye. Journal of pharmaceutical sciences, 1998. 87(12): p. 1479-1488.

265. Shsrma, A. and B. Kuppermann, New and in-development treatments for diabetic macular edema. Retinal physician, 2008. 5(6): p. 20-30. 
266. Cunha-Vaz, J.G., The blood-ocular barriers: Past, present, and future.

Documenta Ophthalmologica, 1997. 93(1-2): p. 149-157.

267. Abbott, N.J., L. Ronnback, and E. Hansson, Astrocyte-endothelial interactions at the blood-brain barrier. Nature Reviews Neuroscience, 2006. 7(1): p. 41-53.

268. Wu, L., et al., Overcoming the blood-brain barrier in chemotherapy treatment of pediatric brain tumors. Pharmaceutical research, 2014. 31(3): p. 531-540.

269. Ballabh, P., A. Braun, and M. Nedergaard, The blood-brain barrier: An overview: Structure, regulation, and clinical implications. Neurobiology of Disease, 2004. 16(1): p. 1-13.

270. Pardridge, W.M., Blood-brain barrier delivery. Drug Discovery Today, 2007. 12(1-2): p. 54-61.

271. Blakeley, J., Drug delivery to brain tumors. Current Neurology and Neuroscience Reports, 2008. 8(3): p. 235-241.

272. Eyal, S., P. Hsiao, and J.D. Unadkat, Drug interactions at the blood-brain barrier: Fact or fantasy? Pharmacology and Therapeutics, 2009. 123(1): p. 80104.

273. Kalvass, J.C., et al., Why clinical modulation of efflux transport at the human blood-brain barrier is unlikely: The ITC evidence-based position. Clinical Pharmacology and Therapeutics, 2013. 94(1): p. 80-94.

274. El-Bacha, R.S. and A. Minn, Drug metabolizing enzymes in cerebrovascular endothelial cells afford a metabolic protection to the brain. Cellular and Molecular Biology, 1999. 45(1): p. 15-23.

275. Kreuter, J., et al., Direct evidence that polysorbate-80-coated poly( butylcyanoacrylate) nanoparticles deliver drugs to the CNS via specific mechanisms requiring prior binding of drug to the nanoparticles. Pharmaceutical Research, 2003. 20(3): p. 409-416.

276. Weiss, C.K., et al., The first step into the brain: Uptake of NIO-PBCA nanoparticles by endothelial cells in vitro and in vivo, and direct evidence for their blood-brain barrier permeation. Chemmedchem, 2008. 3(9): p. 1395-1403.

277. Vergoni, A.V., et al., Nanoparticles as drug delivery agents specific for CNS: in vivo biodistribution. Nanomedicine: Nanotechnology, Biology, and Medicine, 2009. 5(4): p. 369-377.

278. Mogami, H., et al., Selection of cytostatic agents for intrathecal chemotherapy of brain tumor. Medical Journal of Osaka University, 1967. 17(3): p. 333-340.

279. Wilson, C.B. and H.A. Norrell Jr, Brain tumor chemotherapy with intrathecal methotrexate. Cancer, 1969. 23(5): p. 1038-1045.

280. Kerr, J.Z., S. Berg, and S.M. Blaney, Intrathecal chemotherapy. Critical Reviews in Oncology/Hematology, 2001. 37(3): p. 227-236.

281. Lassaletta, A., et al., Intrathecal liposomal cytarabine in children under 4 years with malignant brain tumors. Journal of Neuro-Oncology, 2009. 95(1): p. 65-69.

282. Gururangan, S. and H.S. Friedman, Innovations in design and delivery of chemotherapy for brain tumors. Neuroimaging Clinics of North America, 2002. 12(4): p. 583-597.

283. Bomgaars, L., et al., Phase I trial of intrathecal liposomal cytarabine in children with neoplastic meningitis. Journal of Clinical Oncology, 2004. 22(19): p. 39163921. 
284. Parasole, R., et al., Efficacy and safety of intrathecal liposomal cytarabine for the treatment of meningeal relapse in acute lymphoblastic leukemia: Experience of two pediatric institutions. Leukemia and Lymphoma, 2008. 49(8): p. 1553-1559.

285. Partap, S., et al., Liposomal cytarabine for central nervous system embryonal tumors in children and young adults. Journal of Neuro-Oncology, 2011. 103(3): p. 561-566.

286. Hunt Bobo, R., et al., Convection-enhanced delivery of macromolecules in the brain. Proceedings of the National Academy of Sciences of the United States of America, 1994. 91(6): p. 2076-2080.

287. Allard, E., C. Passirani, and J.-P. Benoit, Convection-enhanced delivery of nanocarriers for the treatment of brain tumors. Biomaterials, 2009. 30(12): p. 2302-2318.

288. Ferguson, S. and M.S. Lesniak, Convection enhanced drug delivery of novel therapeutic agents to malignant brain tumors. Current Drug Delivery, 2007. 4(2): p. 169-180.

289. Lidar, Z., et al., Convection-enhanced delivery of paclitaxel for the treatment of recurrent malignant glioma: A Phase I/II clinical study. Journal of Neurosurgery, 2004. 100(3): p. 472-479.

290. Kunwar, S., et al., Direct intracerebral delivery of cintredekin besudotox (IL13PE38QQR) in recurrent malignant glioma: A report by the cintredekin besudotox intraparenchymal study group. Journal of Clinical Oncology, 2007. 25(7): p. 837844.

291. Kunwar, S., Convection enhanced delivery of IL13-PE38QQR for treatment of recurrent malignant glioma: presentation of interim findings from ongoing phase 1 studies. Acta neurochirurgica. Supplement, 2003. 88: p. 105-111.

292. Debinski, W. and S.B. Tatter, Convection-enhanced delivery for the treatment of brain tumors. Expert Review of Neurotherapeutics, 2009. 9(10): p. 1519-1527.

293. Bruce, J.N., et al., Regression of recurrent malignant gliomas with convectionenhanced delivery of topotecan. Neurosurgery, 2011. 69(6): p. 1272-1279.

294. Saito, R., et al., Regression of recurrent glioblastoma infiltrating the brainstem after convection-enhanced delivery of nimustine hydrochloride: Case report. Journal of Neurosurgery: Pediatrics, 2011. 7(5): p. 522-526.

295. Neuwelt, E.A., et al., Effects of adrenal cortical steroids and osmotic blood-brain barrier opening on methotrexate delivery to gliomas in the rodent: The factor of the blood-brain barrier. Proceedings of the National Academy of Sciences of the United States of America, 1982. 79(14 I): p. 4420-4423.

296. Patrick, J.T., et al., Ultrasound and the blood-brain barrier. Advances in Experimental Medicine and Biology, 1990. 267: p. 369-381.

297. Elliott, P.J., et al., Unlocking the blood-brain barrier: A role for RMP-7 in brain tumor therapy. Experimental Neurology, 1996. 141(2): p. 214-224.

298. Dahlborg, S.A., et al., The potential for complete and durable response in nonglial primary brain tumors in children and young adults with enhanced chemotherapy delivery. Cancer Journal from Scientific American, 1998. 4(2): p. 110-124. 
299. Warren, K.E., et al., Phase I trial of lobradimil (RMP-7) and carboplatin in children with brain tumors. Cancer Chemotherapy and Pharmacology, 2001. 48(4): p. 275-282.

300. Haluska, M. and M.L. Anthony, Osmotic blood-brain barrier modification for the treatment of malignant brain tumors. Clinical journal of oncology nursing, 2004. 8(3): p. 263-267.

301. McDannold, N., N. Vykhodtseva, and K. Hynynen, Blood-Brain Barrier Disruption Induced by Focused Ultrasound and Circulating Preformed Microbubbles Appears to Be Characterized by the Mechanical Index. Ultrasound in Medicine and Biology, 2008. 34(5): p. 834-840.

302. Guillaume, D.J., et al., Intra-arterial chemotherapy with osmotic blood-brain barrier disruption for aggressive oligodendroglial tumors: Results of a phase $i$ study. Neurosurgery, 2010. 66(1): p. 48-58.

303. Liu, H.L., et al., Blood-brain barrier disruption with focused ultrasound enhances delivery of chemotherapeutic drugs for glioblastoma treatment. Radiology, 2010. 255(2): p. 415-425.

304. Shin, B.J., et al., Superselective Intra-Arterial Cerebral Infusion of Novel Agents After Blood-Brain Disruption for the Treatment of Recurrent Glioblastoma Multiforme: A Technical Case Series. Neurosurgery Clinics of North America, 2012. 23(2): p. 323-329.

305. Neuwelt, E.A., J.T. Diehl, and L.H. Vu, Monitoring of methotrexate delivery in patients with malignant brain tumors after osmotic blood-brain barrier disruption. Annals of Internal Medicine, 1981. 94(4 I): p. 449-454.

306. Miyagami, M., et al., Intra-arterial ACNU chemotherapy employing $20 \%$ mannitol osmotic blood-brain barrier disruption for malignant brain tumors. Neurologia Medico-Chirurgica, 1990. 30(8): p. 582-590.

307. Jahnke, K., et al., Intraarterial chemotherapy and osmotic blood-brain barrier disruption for patients with embryonal and germ cell tumors of the central nervous system. Cancer, 2008. 112(3): p. 581-588.

308. Angelov, L., et al., Blood-brain barrier disruption and intra-arterial methotrexate-based therapy for newly diagnosed primary CNS lymphoma: A multi-institutional experience. Journal of Clinical Oncology, 2009. 27(21): p. 3503-3509.

309. Dahlborg, S.A., et al., Non-AIDS primary CNS lymphoma: First example of a durable response in a primary brain tumor using enhanced chemotherapy delivery without cognitive loss and without radiotherapy. Cancer Journal from Scientific American, 1996. 2(3): p. 166-174.

310. Kemper, E.M., et al., Modulation of the blood-brain barrier in oncology: Therapeutic opportunities for the treatment of brain tumours? Cancer Treatment Reviews, 2004. 30(5): p. 415-423.

311. Ford, J., et al., A phase I study of intravenous RMP-7 with carboplatin in patients with progression of malignant glioma. European Journal of Cancer, 1998. 34(11): p. 1807-1811.

312. Emerich, D.F., et al., The development of the bradykinin agonist labradimil as a means to increase the permeability of the blood-brain barrier: From concept to clinical evaluation. Clinical Pharmacokinetics, 2001. 40(2): p. 105-123. 
313. Warren, K., et al., Pharmacokinetics of carboplatin administered with lobradimil to pediatric patients with brain tumors. Cancer Chemotherapy and Pharmacology, 2004. 54(3): p. 206-212.

314. Liu, L., et al., Biologically active core/shell nanoparticles self-assembled from cholesterol-terminated PEG-TAT for drug delivery across the blood-brain barrier. Biomaterials, 2008. 29(10): p. 1509-1517.

315. Dhanikula, R.S., et al., Methotrexate loaded polyether-copolyester dendrimers for the treatment of gliomas: Enhanced efficacy and intratumoral transport capability. Molecular Pharmaceutics, 2008. 5(1): p. 105-116.

316. Li, C. and S. Wallace, Polymer-drug conjugates: Recent development in clinical oncology. Advanced Drug Delivery Reviews, 2008. 60(8): p. 886-898.

317. Li, C., et al., Complete regression of well-established tumors using a novel watersoluble poly(L-glutamic acid)-paclitaxel conjugate. Cancer Research, 1998. 58(11): p. 2404-2409.

318. Costantino, L. and D. Boraschi, Is there a clinical future for polymeric nanoparticles as brain-targeting drug delivery agents? Drug Discovery Today, 2012. 17(7-8): p. 367-378.

319. Malam, Y., M. Loizidou, and A.M. Seifalian, Liposomes and nanoparticles: nanosized vehicles for drug delivery in cancer. Trends in pharmacological sciences, 2009. 30(11): p. 592-599.

320. Webb, M., et al., Sphingomyelin-cholesterol liposomes significantly enhance the pharmacokinetic and therapeutic properties of vincristine in murine and human tumour models. British Journal of Cancer, 1995. 72(4): p. 896-904.

321. Tokes, Z.A., A.K. Stpeteri, and J.A. Todd, Availability of liposome content to the nervous system. Liposomes and the blood-brain barrier. Brain Research, 1980. 188(1): p. 282-286.

322. Jain, P.K., I.H. El-Sayed, and M.A. El-Sayed, Au nanoparticles target cancer. nano today, 2007. 2(1): p. 18-29.

323. Mamaeva, V., et al., Mesoporous silica nanoparticles as drug delivery systems for targeted inhibition of Notch signaling in cancer. Molecular Therapy, 2011. 19(8): p. 1538-1546.

324. Wang, L., W. Zhao, and W. Tan, Bioconjugated silica nanoparticles: development and applications. Nano Research, 2008. 1(2): p. 99-115.

325. Lu, J., et al., Biocompatibility, Biodistribution, and Drug-Delivery Efficiency of Mesoporous Silica Nanoparticles for Cancer Therapy in Animals. Small, 2010. 6(16): p. 1794-1805.

326. Sun, C., J.S. Lee, and M. Zhang, Magnetic nanoparticles in MR imaging and drug delivery. Advanced Drug Delivery Reviews, 2008. 60(11): p. 1252-1265.

327. Fan, C.-H., et al., SPIO-conjugated, doxorubicin-loaded microbubbles for concurrent MRI and focused-ultrasound enhanced brain-tumor drug delivery. Biomaterials, 2013.

328. Koo, Y.E.L., et al., Brain cancer diagnosis and therapy with nanoplatforms. Advanced Drug Delivery Reviews, 2006. 58(14): p. 1556-1577.

329. Jones, A.R. and E.V. Shusta, Blood-brain barrier transport of therapeutics via receptor-mediation. Pharmaceutical Research, 2007. 24(9): p. 1759-1771. 
330. Herve, F., N. Ghinea, and J.M. Scherrmann, CNS Delivery Via Adsorptive Transcytosis. Aaps Journal, 2008. 10(3): p. 455-472.

331. Illum, L., Nasal drug delivery: new developments and strategies. Drug discovery today, 2002. 7(23): p. 1184-1189.

332. Haque, S., et al., Development and evaluation of brain targeted intranasal alginate nanoparticles for treatment of depression. Journal of psychiatric research, 2014. 48(1): p. 1-12.

333. Illum, L., Nanoparticulate systems for nasal delivery of drugs: a real improvement over simple systems? Journal of pharmaceutical sciences, 2007. 96(3): p. 473-483.

334. Losa, C., et al., Improvement of ocular penetration of amikacin sulphate by association to poly(butylcyanoacrylate) nanoparticles. Journal of Pharmacy and Pharmacology, 1991. 43(8): p. 548-552.

335. Losa, C., et al., Design of New Formulations for Topical Ocular Administration: Polymeric Nanocapsules Containing Metipranolol. Pharm Res, 1993. 10(1): p. 80-87.

336. Smolin G, O.M., Feiler S, Condon D., Idoxuridine-liposome therapy for herpes simplex keratitis. Am J Ophthalmol., 1981. 91(2): p. 200-5.

337. Lu, W., et al., Cationic albumin-conjugated pegylated nanoparticles allow gene delivery into brain tumors via intravenous administration. Cancer Research, 2006. 66(24): p. 11878-11887.

338. Régina, A., et al., Antitumour activity of ANG1005, a conjugate between paclitaxel and the new brain delivery vector Angiopep-2. British Journal of Pharmacology, 2008. 155(2): p. 185-197.

339. Thomas, F.C., et al., Uptake of ANG1005, a novel paclitaxel derivative, through the blood-brain barrier into brain and experimental brain metastases of breast cancer. Pharmaceutical Research, 2009. 26(11): p. 2486-2494.

340. Huang, R., et al., Targeted delivery of chlorotoxin-modified DNA-loaded nanoparticles to glioma via intravenous administration. Biomaterials, 2011. 32(9): p. 2399-2406.

341. Nair, B.G., et al., Nanotechnology platforms; an innovative approach to brain tumor therapy. Medicinal Chemistry, 2011. 7(5): p. 488-503.

342. Gaillard, P., et al., Development of glutathione pegylated liposomal doxorubicin (2B3-101) for the treatment of brain cancer. the 4th European Conference for Clinical Nanomedicine, 2011. Basel, Switzerland.

343. Peer, D., et al., Nanocarriers as an emerging platform for cancer therapy. Nat Nano, 2007. 2(12): p. 751-760.

344. Yu, M.K., J. Park, and S. Jon, Targeting strategies for multifunctional nanoparticles in cancer imaging and therapy. Theranostics, 2012. 2(1): p. 3.

345. Huang, H.-C., et al., Inorganic nanoparticles for cancer imaging and therapy. Journal of Controlled Release, 2011. 155(3): p. 344-357.

346. Jallouli, Y., et al., Influence of surface charge and inner composition of porous nanoparticles to cross blood-brain barrier in vitro. International journal of pharmaceutics, 2007. 344(1): p. 103-109. 
347. Fenart, L., et al., Evaluation of effect of charge and lipid coating on ability of 60nm nanoparticles to cross an in vitro model of the blood-brain barrier. Journal of Pharmacology and Experimental Therapeutics, 1999. 291(3): p. 1017-1022.

348. Bose, R.J., L. Soo-Hong, and P. Hansoo, Biofunctionalized nanoparticles: an emerging drug delivery platform for various disease treatments. Drug Discovery Today, 2016.

349. Karatas, H., et al., A nanomedicine transports a peptide caspase-3 inhibitor across the blood-brain barrier and provides neuroprotection. The Journal of Neuroscience, 2009. 29(44): p. 13761-13769.

350. Pardridge, W.M., J.L. Buciak, and P.M. Friden, Selective transport of an antitransferrin receptor antibody through the blood-brain barrier in vivo. Journal of Pharmacology and Experimental Therapeutics, 1991. 259(1): p. 66-70.

351. Lee, H.J., et al., Targeting rat anti-mouse transferrin receptor monoclonal antibodies through blood-brain barrier in mouse. Journal of Pharmacology and Experimental Therapeutics, 2000. 292(3): p. 1048-1052.

352. Debele, T.A., S.L. Mekuria, and H.-C. Tsai, Polysaccharide based nanogels in the drug delivery system: Application as the carrier of pharmaceutical agents. Materials Science and Engineering: C, 2016.

353. Lee, J.W., J.H. Park, and J.R. Robinson, Bioadhesive-based dosage forms: The next generation. Journal of Pharmaceutical Sciences, 2000. 89(7): p. 850-866.

354. Felt, O., et al., Topical use of chitosan in ophthalmology: tolerance assessment and evaluation of precorneal retention. Int J Pharm, 1999. 180(2): p. 185-193.

355. de Salamanca, A.E., et al., Chitosan Nanoparticles as a Potential Drug Delivery System for the Ocular Surface: Toxicity, Uptake Mechanism and In Vivo Tolerance. Invest Ophthalmol Vis Sci, 2006. 47(4): p. 1416-1425.

356. Nagarwal, R.C., et al., Chitosan Nanoparticles of 5-Fluorouracil for Ophthalmic Delivery: Characterization, characterization, in-vitro and in-vivo study. Chemical and Pharmaceutical Bulletin, 2011. 59(2): p. 272-278.

357. Hirano S, S.H., Akiyama I, Nonaka I., Chitosan: a biocompatible material for oral and intravenous administration. Progress in Biomedical Polymers, ed. D.R.L. Gebelein C G. 1990, New York: Plenum Press.

358. Artursson, P., et al., Effect of chitosan on the permeability of monolayers of intestinal epithelial cells (Caco-2). Pharm Res, 1994. 11(9): p. 1358-1361.

359. Takeuchi, H., et al., Enteral absorption of insulin in rats from mucoadhesive Chitosan-Coated liposomes. Pharm Res, 1996. 13(6): p. 896-901.

360. Lemarchand, C., R. Gref, and P. Couvreur, Polysaccharide-decorated nanoparticles. European Journal of Pharmaceutics and Biopharmaceutics, 2004. 58(2): p. 327-341.

361. Alonso, M.J. and A. Sánchez, The potential of chitosan in ocular drug delivery. Journal of Pharmacy and Pharmacology, 2003. 55(11): p. 1451-1463.

362. Agnihotri, S.A., N.N. Mallikarjuna, and T.M. Aminabhavi, Recent advances on chitosan-based micro- and nanoparticles in drug delivery. Journal of Controlled Release, 2004. 100(1): p. 5-28.

363. Nagpal, K., S.K. Singh, and D.N. Mishra, Chitosan Nanoparticles: A Promising System in Novel Drug Delivery. Chemical and Pharmaceutical Bulletin, 2010. 58(11): p. 1423-1430. 
364. Stefania Racovita, S.V., Marcel Popa, Cornelia Luca, Polysaccharides based on micro- and nanoparticles obtained by ionic gelation and their applications as drug delivery systems. Revue Roumaine de Chimie, 2009. 59(9): p. 709-718.

365. Costa, J., et al., Potential chitosan-coated alginate nanoparticles for ocular delivery of daptomycin. European Journal of Clinical Microbiology \& Infectious Diseases, 2015. 34(6): p. 1255-1262.

366. Molnár, E.v., et al., Toward drug delivery into the brain: synthesis, characterization, and preliminary in vitro assessment of alkylglycerylfunctionalized chitosan nanoparticles. Biomacromolecules, 2010. 11(11): p. 2880-2889.

367. Lalatsa, A., et al., Delivery of peptides to the blood and brain after oral uptake of quaternary ammonium palmitoyl glycol chitosan nanoparticles. Molecular pharmaceutics, 2012. 9(6): p. 1764-1774.

368. Motwani, S.K., et al., Chitosan-sodium alginate nanoparticles as submicroscopic reservoirs for ocular delivery: Formulation, optimisation and in vitro characterisation. European Journal of Pharmaceutics and Biopharmaceutics, 2008. 68(3): p. 513-525.

369. Wadhwa, S., et al., Hyaluronic acid modified chitosan nanoparticles for effective management of glaucoma: development, characterization, and evaluation. Journal of Drug Targeting, 2010. 18(4): p. 292-302.

370. Mahmoud, A.A., et al., Chitosan/sulfobutylether-beta-cyclodextrin nanoparticles as a potential approach for ocular drug delivery. Int J Pharm, 2011. 413(1-2): p. 229-236.

371. Kao, H.J., et al., Characterization of pilocarpine-loaded chitosan/Carbopol nanoparticles. Journal of Pharmacy and Pharmacology, 2006. 58(2): p. 179-186.

372. Chaiyasan, W., S.P. Srinivas, and W. Tiyaboonchai, Mucoadhesive ChitosanDextran Sulfate Nanoparticles for Sustained Drug Delivery to the Ocular Surface. Journal of Ocular Pharmacology and Therapeutics, 2013. 29(2): p. 200-207.

373. Chhonker, Y.S., et al., Amphotericin-B entrapped lecithin/chitosan nanoparticles for prolonged ocular application. International journal of biological macromolecules, 2014.

374. Sinha, V.R. and R. Kumria, Polysaccharides in colon-specific drug delivery. International Journal of Pharmaceutics, 2001. 224(1-2): p. 19-38.

375. Rubinstein, A., Natural polysaccharides as targeting tools of drugs to the human colon. Drug Development Research, 2000. 50(3-4): p. 435-439.

376. Vandamme, T.F., et al., The use of polysaccharides to target drugs to the colon. Carbohydrate Polymers, 2002. 48(3): p. 219-231.

377. Kaur, H., et al., Carboxymethyl tamarind kernel polysaccharide nanoparticles for ophthalmic drug delivery. Int J Biol Macromol, 2012. 50(3): p. 833-9.

378. Yadav, M. and M. Ahuja, Preparation and evaluation of nanoparticles of gum cordia, an anionic polysaccharide for ophthalmic delivery. Carbohydrate Polymers, 2010. 81(4): p. 871-877.

379. Sharma, R., M. Ahuja, and H. Kaur, Thiolated pectin nanoparticles: Preparation, characterization and ex vivo corneal permeation study. Carbohydrate Polymers, 2012. 87(2): p. 1606-1610. 
380. Horvát, G., et al., Comparative study of nanosized cross-linked sodium-, linear sodium-and zinc-hyaluronate as potential ocular mucoadhesive drug delivery systems. International journal of pharmaceutics, 2015. 494(1): p. 321-328.

381. Boridy, S., et al., The binding of pullulan modified cholesteryl nanogels to $A \beta$ oligomers and their suppression of cytotoxicity. Biomaterials, 2009. 30(29): p. 5583-5591.

382. Morimoto, N., et al., Design of hybrid hydrogels with self-assembled nanogels as cross-linkers: interaction with proteins and chaperone-like activity.

Biomacromolecules, 2005. 6(4): p. 1829-1834.

383. Kaur, A., S. Jain, and A. Tiwary, Mannan-coated gelatin nanoparticles for sustained and targeted delivery of didanosine: in vitro and in vivo evaluation. Acta pharmaceutica, 2008. 58(1): p. 61-74.

384. Lv, W., et al., Temperature-and Redox-Directed Multiple Self Assembly of Poly (N-Isopropylacrylamide) Grafted Dextran Nanogels. Macromolecular rapid communications, 2011. 32(14): p. 1101-1107.

385. Wang, L.-Q., et al., Synthesis and characterization of temperature responsive graft copolymers of dextran with poly ( $N$-isopropylacrylamide). Reactive and Functional Polymers, 2002. 53(1): p. 19-27.

386. Boddohi, S. and M.J. Kipper, Engineering nanoassemblies of polysaccharides. Advanced Materials, 2010. 22(28): p. 2998-3016.

387. Zhang, X., D. Wu, and C.-C. Chu, Synthesis and characterization of partially biodegradable, temperature and pH sensitive Dex-MA/PNIPAAm hydrogels. Biomaterials, 2004. 25(19): p. 4719-4730.

388. Hovgaard, L. and H. Brøndsted, Dextran hydrogels for colon-specific drug delivery. Journal of Controlled Release, 1995. 36(1): p. 159-166.

389. Mitra, S., et al., Tumour targeted delivery of encapsulated dextran-doxorubicin conjugate using chitosan nanoparticles as carrier. Journal of Controlled Release, 2001. 74(1): p. 317-323.

390. Chiu, H.-C., et al., Synthesis and characterization of pH-sensitive dextran hydrogels as a potential colon-specific drug delivery system. Journal of Biomaterials Science, Polymer Edition, 1999. 10(5): p. 591-608.

391. Zhang, R., Synthesis, characterization and reversible transport of thermosensitive carboxyl methyl dextran/poly ( $N$-isopropylacrylamide) hydrogel. Polymer, 2005. 46(8): p. 2443-2451.

392. Rousseau, V., et al., Investigation of blood-brain barrier permeability to magnetite-dextran nanoparticles (MD3) after osmotic disruption in rats. Magnetic Resonance Materials in Physics, Biology and Medicine, 1997. 5(3): p. 213-222.

393. Delgado, D., et al., Dextran and protamine-based solid lipid nanoparticles as potential vectors for the treatment of $X$-linked juvenile retinoschisis. Human gene therapy, 2012. 23(4): p. 345-355.

394. Zorzi, G.K., et al., Hybrid nanoparticle design based on cationized gelatin and the polyanions dextran sulfate and chondroitin sulfate for ocular gene therapy. Macromolecular bioscience, 2011. 11(7): p. 905-913. 
395. Liu, S., L. Jones, and F.X. Gu, Development of Mucoadhesive Drug Delivery System Using Phenylboronic Acid Functionalized Poly (D, L-lactide)-b-Dextran Nanoparticles. Macromolecular bioscience, 2012. 12(12): p. 1622-1626.

396. Mykhaylyk, O., et al., Glial brain tumor targeting of magnetite nanoparticles in rats. Journal of magnetism and magnetic materials, 2001. 225(1): p. 241-247.

397. Liu, S., et al., Phenylboronic acid modified mucoadhesive nanoparticle drug carriers facilitate weekly treatment of experimentallyinduced dry eye syndrome. Nano Research, 2015. 8(2): p. 621-635.

398. Schroeder, U., P. Sommerfeld, and B.A. Sabel, Efficacy of oral dalargin-loaded nanoparticle delivery across the blood-brain barrier. Peptides, 1998. 19(4): p. 777-780.

399. Szejtli, J., Introduction and general overview of cyclodextrin chemistry. Chemical Reviews, 1998. 98(5): p. 1743-1753.

400. Hirayama, F. and K. Uekama, Cyclodextrin-based controlled drug release system. Adv. Drug. Deliv. Rev., 1999. 36(1): p. 125-141.

401. Challa, R., et al., Cyclodextrins in drug delivery: an updated review. Aaps Pharmscitech, 2005. 6(2): p. E329-E357.

402. Rajewski, R.A. and V.J. Stella, Pharmaceutical applications of cyclodextrins. 2. In vivo drug delivery. Journal of pharmaceutical sciences, 1996. 85(11): p. 11421169.

403. Tao, H.-q., et al., HP- $\beta-C D-P L G A$ nanoparticles improve the penetration and bioavailability of puerarin and enhance the therapeutic effects on brain ischemiareperfusion injury in rats. Naunyn-Schmiedeberg's archives of pharmacology, 2013. 386(1): p. 61-70.

404. Barber, A.J., A new view of diabetic retinopathy: a neurodegenerative disease of the eye. Progress in Neuro-Psychopharmacology and Biological Psychiatry, 2003. 27(2): p. 283-290.

405. Barber, A.J., et al., Insulin rescues retinal neurons from apoptosis by a phosphatidylinositol 3-kinase/Akt-mediated mechanism that reduces the activation of caspase-3. Journal of Biological Chemistry, 2001. 276(35): p. 32814-32821.

406. Wu, X., et al., Insulin promotes rat retinal neuronal cell survival in a p70S6Kdependent manner. Journal of Biological Chemistry, 2004. 279(10): p. 91679175.

407. Reiter, C.E., et al., Characterization of insulin signaling in rat retina in vivo and ex vivo. American Journal of Physiology-Endocrinology and Metabolism, 2003. 285(4): p. E763-E774.

408. Misra, G.P., et al., Subconjunctivally implantable hydrogels with degradable and thermoresponsive properties for sustained release of insulin to the retina. Biomaterials, 2009. 30(33): p. 6541-6547.

409. Imai, H., et al., Subconjunctivally Implanted Hydrogels for Sustained Insulin Release to Reduce Retinal Cell Apoptosis in Diabetic RatsHydrogels for Sustained Retinal Delivery of Insulin. Investigative ophthalmology \& visual science, 2015. 56(13): p. 7839-7846.

410. Henry, W., H. Querfurth, and F. LaFerla, Mechanisms of disease Alzheimer's disease. New Engl J Med, 2010. 362: p. 329-44. 
411. Association, A.s., 2016 Alzheimer's disease facts and figures. Alzheimer's \& Dementia, 2016. 12(4): p. 459-509.

412. Kim, J.-Y., et al., Tip-loaded dissolving microneedles for transdermal delivery of donepezil hydrochloride for treatment of Alzheimer's disease. European Journal of Pharmaceutics and Biopharmaceutics, 2016.

413. O'Brien, R.J. and P.C. Wong, Amyloid precursor protein processing and Alzheimer's disease. Annual review of neuroscience, 2011. 34: p. 185.

414. Ho, S.W., et al., Effects of 17-allylamino-17-demethoxygeldanamycin (17-AAG) in transgenic mouse models of frontotemporal lobar degeneration and Alzheimer's disease. Translational neurodegeneration, 2013. 2(1): p. 1.

415. Waza, M., et al., Modulation of Hsp90 function in neurodegenerative disorders: a molecular-targeted therapy against disease-causing protein. Journal of molecular medicine, 2006. 84(8): p. 635-646.

416. Muchowski, P.J. and J.L. Wacker, Modulation of neurodegeneration by molecular chaperones. Nature Reviews Neuroscience, 2005. 6(1): p. 11-22.

417. Klettner, A., The induction of heat shock proteins as a potential strategy to treat neurodegenerative disorders. Drug News Perspect, 2004. 17(5): p. 299-306.

418. Luo, W., et al., Heat shock protein 90 in neurodegenerative diseases. Molecular neurodegeneration, 2010. 5(1): p. 1.

419. Banks, W.A., Drug delivery to the brain in Alzheimer's disease: Consideration of the blood-brain barrier. Advanced drug delivery reviews, 2012. 64(7): p. 629639.

420. Abdallah, B., et al., A powerful nonviral vector for in vivo gene transfer into the adult mammalian brain: polyethylenimine. Human gene therapy, 1996. 7(16): p. 1947-1954.

421. dos Santos, A.L.G., et al., Sustained release of nanosized complexes of polyethylenimine and anti-TGF- $\beta 2$ oligonucleotide improves the outcome of glaucoma surgery. Journal of controlled release, 2006. 112(3): p. 369-381.

422. Liu, Y., et al., A leptin derived 30-amino-acid peptide modified pegylated poly-Llysine dendrigraft for brain targeted gene delivery. Biomaterials, 2010. 31(19): p. 5246-5257.

423. Huang, X. and T.L. Lowe, Biodegradable thermoresponsive hydrogels for aqueous encapsulation and controlled release of hydrophilic model drugs. Biomacromolecules, 2005. 6(4): p. 2131-2139.

424. Gil, E.S., et al., $\beta$-Cyclodextrin-poly( $\beta$-amino ester) Nanoparticles for Drug Delivery across the Blood-Brain Barrier. Biomacromolecules, 2012. In press.

425. Anderson, O.A., et al., Human transscleral albumin permeability and the effect of topographical location and donor age. Investigative ophthalmology \& visual science, 2008. 49(9): p. 4041-4045.

426. Huang, X., et al., Novel nanogels with both thermoresponsive and hydrolytically degradable properties. Macromolecules, 2008. 41(22): p. 8339-8345.

427. Xu, Q., S.P. Kambhampati, and R.M. Kannan, Nanotechnology approaches for ocular drug delivery. Middle East African journal of ophthalmology, 2013. 20(1): p. 26.

428. Kelf, T., et al., Non-specific cellular uptake of surface-functionalized quantum dots. Nanotechnology, 2010. 21(28): p. 285105. 
429. Perumal, O.P., et al., The effect of surface functionality on cellular trafficking of dendrimers. Biomaterials, 2008. 29(24): p. 3469-3476.

430. Zhang, S., et al., Size-Dependent Endocytosis of Nanoparticles. Advanced Materials, 2009. 21(4): p. 419-424.

431. Vácha, R., F.J. Martinez-Veracoechea, and D. Frenkel, Receptor-mediated endocytosis of nanoparticles of various shapes. Nano letters, 2011. 11(12): $\mathrm{p}$. 5391-5395.

432. Harush-Frenkel, O., et al., Surface charge of nanoparticles determines their endocytic and transcytotic pathway in polarized MDCK cells.

Biomacromolecules, 2008. 9(2): p. 435-443.

433. Chen, Y., et al., Hsp90 chaperone inhibitor 17-AAG attenuates A $\beta$-induced synaptic toxicity and memory impairment. The Journal of Neuroscience, 2014. 34(7): p. 2464-2470.

434. Guo, W., D. Siegel, and D. Ross, Stability of the Hsp90 inhibitor $17 A A G$ hydroquinone and prevention of metal-catalyzed oxidation. Journal of pharmaceutical sciences, 2008. 97(12): p. 5147-5157.

435. Chaudhary, V. and J. Patel, Cyclodextrin inclusion complex to enhance solubility of poorly water soluble drugs: A review. International Journal of Pharmaceutical Sciences and Research, 2013. 4(1): p. 68.

436. Béduneau, A., P. Saulnier, and J.-P. Benoit, Active targeting of brain tumors using nanocarriers. Biomaterials, 2007. 28(33): p. 4947-4967.

437. Hoffman, A.S., Hydrogels for biomedical applications. Advanced drug delivery reviews, 2012. 64: p. 18-23.

438. Peppas, N., et al., Physicochemical foundations and structural design of hydrogels in medicine and biology. Annual review of biomedical engineering, 2000. 2(1): p. 9-29.

439. Qiu, Y. and K. Park, Environment-sensitive hydrogels for drug delivery. Advanced drug delivery reviews, 2001. 53(3): p. 321-339.

440. Vermonden, T., R. Censi, and W.E. Hennink, Hydrogels for protein delivery. Chemical Reviews, 2012. 112(5): p. 2853-2888.

441. Paradiso, P., et al., Comparison of two hydrogel formulations for drug release in ophthalmic lenses. Journal of Biomedical Materials Research Part B: Applied Biomaterials, 2014.

442. Radhakrishnan, J., U.M. Krishnan, and S. Sethuraman, Hydrogel based injectable scaffolds for cardiac tissue regeneration. Biotechnology advances, 2014.

443. Rezaei, S. and Z. Ishak, Grafting of collagen onto interpenetrating polymer networks of poly (2-hydroxyethyl methacrylate) and poly (dimethyl siloxane) polymer films for biomedical applications. Express Polymer Letters, 2014. 8(1).

444. Yom-Tov, O., et al., A novel method for hydrogel nanostructuring. European Polymer Journal, 2014.

445. Wilson, A.N. and A. Guiseppi-Elie, Targeting homeostasis in drug delivery using bioresponsive hydrogel microforms. International journal of pharmaceutics, 2014. 461(1): p. 214-222.

446. Kotanen, C.N., et al., Biomimetic hydrogels gate transport of calcium ions across cell culture inserts. Biomedical microdevices, 2012. 14(3): p. 549-558. 
447. Lewis, C.L. and M. Anthamatten, Synthesis, swelling behavior, and viscoelastic properties of functional poly(hydroxyethyl methacrylate) with ureidopyrimidinone side-groups. Soft Matter, 2013.9(15): p. 4058-4066.

448. Morita, S., Hydrogen-bonds structure in poly(2-hydroxyethyl methacrylate) studied by temperature-dependent infrared spectroscopy. Frontiers in Chemistry, 2014. 2: p. 10.

449. Lord, M.S., et al., The effect of charged groups on protein interactions with poly(HEMA) hydrogels. Biomaterials, 2006. 27(4): p. 567-575.

450. Wilson, A., M. Blenner, and A. Guiseppi-Elie, Polyplex Formation Influences Release Mechanism of Mono- and Di-Valent Ions from Phosphorylcholine Group Bearing Hydrogels. Polymers, 2014. 6(9): p. 2451-2472.

451. Schillemans, J.P., et al., Anionic and cationic dextran hydrogels for post-loading and release of proteins. Journal of controlled release, 2011. 150(3): p. 266-271.

452. Lin, C.-C. and A.T. Metters, Hydrogels in controlled release formulations: network design and mathematical modeling. Advanced drug delivery reviews, 2006. 58(12): p. 1379-1408.

453. Cadée, J.A., et al., Oxidation of recombinant human interleukin-2 by potassium peroxodisulfate. Pharmaceutical research, 2001. 18(10): p. 1461-1467.

454. Kim, S.W., Y.H. Bae, and T. Okano, Hydrogels: swelling, drug loading, and release. Pharmaceutical Research, 1992. 9(3): p. 283-290.

455. Zhang, Y., et al., A novel microgel and associated post-fabrication encapsulation technique of proteins. Journal of controlled release, 2005. 105(3): p. 260-268.

456. Gehrke, S.H., L.H. Uhden, and J.F. McBride, Enhanced loading and activity retention of bioactive proteins in hydrogel delivery systems. Journal of Controlled Release, 1998. 55(1): p. 21-33.

457. Gehrke, S.H., N.R. Vaid, and J.F. McBride, Protein sorption and recovery by hydrogels using principles of aqueous two-phase extraction. Biotechnology and bioengineering, 1998. 58(4): p. 416-427.

458. Pan, G., et al., Dynamic introduction of cell adhesive factor via reversible multicovalent phenylboronic acid/cis-diol polymeric complexes. Journal of the American Chemical Society, 2014. 136(17): p. 6203-6206.

459. Bayramoğlu, G., et al., Heparin-coated poly (hydroxyethyl methacrylate/albumin) hydrogel networks: In vitro hemocompatibility evaluation for vascular biomaterials. Journal of applied polymer science, 2008. 109(2): p. 749-757.

460. Liu, J., et al., Aptamer-incorporated hydrogels for visual detection, controlled drug release, and targeted cancer therapy. Analytical and bioanalytical chemistry, 2012. 402(1): p. 187-194.

461. Perçin, I., et al., Poly (hydroxyethyl methacrylate) based affinity cryogel for plasmid DNA purification. International journal of biological macromolecules, 2011. 48(4): p. 577-582.

462. Lai, R., et al., An anionic antimicrobial peptide from toad Bombina maxima. Biochemical and biophysical research communications, 2002. 295(4): p. 796-799.

463. Kotanen, C.N., et al., Partitioning of coomassie brilliant blue into DMAEMA containing poly (HEMA)-based hydrogels. European Polymer Journal, 2015. 72: p. 438-450. 
464. Abdurrahmanoglu, S., V. Can, and O. Okay, Design of high-toughness polyacrylamide hydrogels by hydrophobic modification. Polymer, 2009. 50(23): p. 5449-5455.

465. Gabbott, P., Principles and applications of thermal analysis. 2008: John Wiley \& Sons.

466. Immergut, E.H. and H.F. Mark, Principles of plasticization. 1965.

467. Krumova, M., et al., Effect of crosslinking on the mechanical and thermal properties of poly (vinyl alcohol). Polymer, 2000. 41(26): p. 9265-9272.

468. Peppas, N.A., Kinetics of Smart Hydrogels, in Reflexive Polymers and Hydrogels: Understanding and Designing Fast Responsive Polymeric Systems, N. Yui, R.J. Mrsny, and K. Park, Editors. 2004, CRC Press Taylor and Francis Division: New York. p. 472.

469. Li, W., F. Xue, and R. Cheng, States of water in partially swollen poly (vinyl alcohol) hydrogels. Polymer, 2005. 46(25): p. 12026-12031.

470. Ostrowska-Czubenko, J., M. Pieróg, and M. Gierszewska-Drużyńska, State of water in noncrosslinked and crosslinked hydrogel chitosan membranes-DSC studies. Progr. Chem. Appl. Chitin Deriv., 2011. 16: p. 147-156.

471. Ping, Z.H., et al., States of water in different hydrophilic polymers - DSC and FTIR studies. Polymer, 2001. 42(20): p. 8461-8467.

472. Yudianti, R., et al., DSC analysis on water state of salvia hydrogels. Macromolecular Research, 2009. 17(12): p. 1015-1020.

473. Tanaka, M. and A. Mochizuki, Effect of water structure on blood compatibilitythermal analysis of water in poly(meth)acrylate. Journal of Biomedical Materials Research Part A, 2004. 68A(4): p. 684-695.

474. Topgaard, D. and O. Söderman, Diffusion of Water Absorbed in Cellulose Fibers Studied with 1H-NMR. Langmuir, 2001. 17(9): p. 2694-2702.

475. Topgaard, D. and O. Söderman, Self-Diffusion of Nonfreezing Water in Porous Carbohydrate Polymer Systems Studied with Nuclear Magnetic Resonance. Biophysical Journal, 2002. 83(6): p. 3596-3606.

476. Çaykara, T., et al., Influence of gel composition on the solubility parameter of poly (2-hydroxyethyl methacrylate-itaconic acid) hydrogels. Journal of Polymer Science Part B: Polymer Physics, 2002. 40(18): p. 1995-2003.

477. Kwok, A.Y., G.G. Qiao, and D.H. Solomon, Synthetic hydrogels 3. Solvent effects on poly (2-hydroxyethyl methacrylate) networks. Polymer, 2004. 45(12): p. 40174027.

478. Mandal, S. and V.G. Pangarkar, Pervaporative dehydration of 1-methoxy propanol with acrylonitrile based co-polymer membranes prepared through emulsion polymerization: a solubility parameter approach and study of structural impact. Journal of membrane science, 2002. 209(1): p. 53-66.

479. Shang, K., G. Shan, and P. Pan, Unique multiple soluble-insoluble phase transitions in aqueous two-phase copolymerization of acrylamide and a weakly charged comonomer. Soft matter, 2014. 10(44): p. 8913-8922.

480. Hennink, W. and C.F. Van Nostrum, Novel crosslinking methods to design hydrogels. Advanced drug delivery reviews, 2012. 64: p. 223-236. 
481. Song, G., et al., Facile fabrication of tough hydrogels physically cross-linked by strong cooperative hydrogen bonding. Macromolecules, 2013. 46(18): p. 74237435. 


\section{APPENDIX. MECHANICAL, THERMAL AND HYDRATION CHARACTERIZATION OF POLY (HEMA)-BASED HYDROGELS*}

\section{Introduction}

Hydrogels are polymeric materials comprised of hydrophilic and hydrophobic components that assist in closely controlling physicochemical properties [437, 438]. They have made a significant contribution to the field of biomedical sciences through various applications such as delivery systems of therapeutics or as scaffolds for tissue engineering or to fill a defect $[437,439,440]$. Poly(2-hydroxyethyl methacrylate) [poly(HEMA)] based hydrogels have gained extensive attention because of their inherent biocompatibility, hydrophilicity, and synthetic flexibility. Synthesis of poly(HEMA) based hydrogels readily permits incorporation of various other components (such as monomers/prepolymers) containing unique functional groups for their use in novel industrial formulations and biomedical applications [441-444]. They have been used as constructs for the effective hosting of biomolecules, whole cell encapsulation, soft contact lenses and membranes that confer biocompatibility to in vivo biosensors.

The physicochemical properties of the hydrogels such as mechanical, thermal and hydration characteristics are typically controlled through bifunctional crosslinking agents such as oligo- and poly- ethylene glycol diacrylates e.g. tetra(ethylene glycol) diacrylate (TEGDA), which, when added at varying concentrations, influence the modulus and the void volume through the cross-link density of the hydrogel $[445,446]$. However, such crosslinks may, in addition, be virtual and thus arise from hydrogen bonding [447, 448], electrostatic interactions[449], polyplex formation [450] and molecular physical entanglements. The physicochemical properties of the hydrogel systems such as mechanical, thermal and hydration characteristics play a significant role in deciding the fate and overall functionally of the hydrogels. As these systems are implanted at various physiological locations in the body, it is important to mimic the local tissue mechanical environment and should be able to withstand the shear and compression forces excreted by the surrounded tissues. Inappropriate mechanical properties may lead to failure of the system. Thermal properties of the system such as glass transition temperature $\left(T_{g}\right)$ are also critical for biomedical applications especially in terms of considerations of processing, handling quality and storage.

Hydrogels have shown their potential as drug delivery systems for controlled release of biological therapeutics such as peptides and proteins, primarily due to their high hydrophilicity, tissue similarity and chance to tailor the drug release [439, 440, 451].

\footnotetext{
* Adapted with permission from (ii) "Partitioning of coomassie brilliant blue into DMAEMA containing poly(HEMA)-based hydrogels 72. Kotanen C.N., Janagam, D.R., Idziak, R., Rhym, L., Sullivan, R., Wilson, A.M., Lowe, T.L., GuiseppiElie, A. European Polymer Journal. p. 438-450. C 2015 Elsevier Ltd., with permission from Elsevier."
} 
Therapeutics can be loaded into the hydrogels either during the hydrogel synthesis (preloading) or after the synthesis of hydrogels (post-loading). Pre-loading of biologics may expose them to various harsh conditions and crosslinking agents which may either damage the drugs or covalently graft them to the polymeric network [451-453]. Therefore post loading can be more favorable for loading of therapeutics specifically into the hydrogels to preserve their activity. Some of the commonly used post loading techniques are solvent sorption where the hydrogel is soaked in drug or drug/polymer, and drug gets into the system by driving forces such as swelling or partitioning in salt-rich phase, but these techniques has shown poor loading and fast washing out of the loaded drug [452, 454-457]. Another alternative way of achieving post loading is by exploiting the reversible electrostatic interactions between the oppositely charged units such as immobilization of charged proteins in oppositely charged hydrogels at low ionic strength and released at physiological ionic strength. This post-loading technique has shown to be very promising in attaining high efficiency and controlled release of biologics [451].

Poly(HEMA)-hydrogels can be readily molecularly engineered to contain a wide variety of pendant and crosslinking bioactive moieties that render them biologically responsive wherein the response of the hydrogel is triggered by recognition of a biological agent conferred by an immobilized biorecognition species. These hydrogels can be easily modified to possess a positive charge, and there are several negatively charged biomolecules that can have potential applications in bioactive poly(HEMA) hydrogels such as cell adhesive factors [458], heparin [459], DNA aptamers [460], plasmids [461], and antimicrobial peptides [462]. The cationic charge can be introduced into the poly(HEMA)-hydrogels by copolymerization with carcinogenic monomers such as dimethyl amino ethyl methacrylate (DMAEMA) or aminoethyl methacrylate (AEMA) and the hydrogels can be employed for encapsulating biomolecules by post-loading technique. However, incorporation of these additional components into the poly(HEMA) structure may affect the physicochemical properties of the hydrogels. Therefore, the objective of this study was to evaluate the impact of ionizable modifiers DMAEMA on mechanical, thermal and hydration properties of the poly(HEMA)-based hydrogels.

\section{Materials and Methods}

\section{Chemicals and Reagents}

Octadecyltrichlorosilane (OTS), 2-hydroxyethyl methacrylate (HEMA), tetra(ethylene glycol) diacrylate (TEGDA, technical grade), oligo(ethylene glycol)(400)methacrylate (OEG(400)MA), N [Tris(hydroxymethyl)methyl]acrylamide (HMMA, 93\%), polyvinylpyrrolidone (pNVP, Mw 1,300,000), 2-(Dimethylamino)ethyl methacrylate (DMAEMA, 98\%), the photo-initiator 2,2-Dimethoxy-2phenylacetophenone (DMPA, 99+\%), and all other common solvents and buffers were purchased from Sigma-Aldrich Co. (St. Louis, MO, USA). HEPES buffer was prepared at $0.025 \mathrm{M}$ and $\mathrm{pH}=7.35$. The HEMA, methacrylate, and diacrylate reagents were passed over an inhibitor removal column (Sigma-Aldrich) for removal of the 
polymerization inhibitors hydroquinone and monomethyl ether hydroquinone before using them in the preparation of the hydrogel cocktail. Solutions were prepared in deionized water prepared by purifying distilled water through a Milli-Q ${ }^{\circledR}$ plus (Millipore Inc.) ultrapure water system.

\section{Synthesis of Poly(HEMA)-based Hydrogels}

The composition of the hydrogels is given in Table A-1. 5 mol\% of charged modifiers either DMAEMA or AMEA were incorporated into the hydrogels. Our collaborators synthesized the hydrogels with and without the charge modifiers according to the procedure published [463]. The resulting structures are schematically illustrated in Figure A-1.

\section{Mechanical Characterization of Hydrogels}

Rheological and compression measurements were performed on poly(HEMA)based hydrogel formulated with and without $5 \mathrm{~mol} \%$ DMAEMA. Samples were run using the simple parallel plate geometry on an AR-G2 Rheometer (TA Instruments). Following equilibration in deionized water, hydrogel samples $(\phi=4.5 \mathrm{~mm}$ and $\mathrm{t}=1.0 \mathrm{~mm})$ were first subjected to an oscillating strain sweep survey test to define the linear viscoelastic (LVE) region wherein the storage modulus $\left(\mathrm{G}^{\prime}\right)$ and loss modulus $\left(\mathrm{G}^{\prime \prime}\right)$ were independent of the applied strain. The hydrogels were then subjected to a frequency ramp from 0.1 to $30 \mathrm{~Hz}$ at a constant strain (25\%) from the LVE region while maintaining the temperature at $37^{\circ} \mathrm{C}$ and the sampling rate of 10 points per decade. The resulting graph of $\mathrm{G}^{\prime}$ and $\mathrm{G}^{\prime \prime}$ over the range of frequency was created at the end of the procedure.

The compression profiles of the equilibrated and swollen hydrogels were evaluated with the compression clamp using a Dynamic Mechanical Analyzer (DMA) Q800 (TA Instruments). The DMA was calibrated before each set of experiments, and the dimensions of the hydrogels were measured and recorded in the accompanying DMA software before testing. Starting at a normal force of $0.001 \mathrm{~N}$, the hydrogel was heated to $37^{\circ} \mathrm{C}$ and put under a force ramp of $0.25 \mathrm{~N} / \mathrm{min}$ until the machine reached $8 \mathrm{~N}$. After the force ramp was complete, a graph of the resulting stress/strain curve was produced. To determine Young's modulus of the compressed hydrogel, the slope of the stress/strain curve was analyzed at a point where the profile was linear and most consistent. Compression profiles of the hydrogels under a compression ramp that went up to $18 \mathrm{~N}$ (maximum force) was used to determine the rupture force the hydrogels.

\section{Glass Transition Temperature $\left(T_{g}\right)$ of the Hydrogels}

$T_{g}$ of the hydrogels with and without 5 mol\% DMAEMA was determined by thermal analysis using a DSC Q2000 (TA Instruments). Around 7 to $10 \mathrm{mg}$ of dried

hydrogel was cut, placed into individual hermetically sealed aluminum pans (Tzero 
Table A-1. Monomer cocktail formulations for the preparation of varying mol\% TEGDA crosslinked poly(HEMA)-based hydrogels prepared in triplicate.

\begin{tabular}{lccc}
\hline Crosslink density (mol\%) & Control & $\begin{array}{c}\text { DMAEMA } \\
\text { modified }\end{array}$ & $\begin{array}{c}\text { AEMA } \\
\text { modified }\end{array}$ \\
\hline HEMA & 86 & 79 & 79 \\
TEGDA & 1 & 3 & 3 \\
OEG(400)MA (n=10) & 5 & 5 & 5 \\
HMMA & 5 & 5 & 5 \\
pNVP & 2 & 2 & 2 \\
DMAEMA/AEMA & 0 & 5 & 5 \\
DMPA & 1 & 1 & 1 \\
\hline
\end{tabular}

Notes: HEMA, 2-hydroxyethyl methacrylate; TEGDA, tetra(ethylene glycol) diacrylate; OEG, oligo(ethylene glycol) methacrylate; pNVP, polyvinylpyrrolidone; HMMA, N[Tris(hydroxymethyl)methyl]acrylamide; DMAEMA, 2-(dimethylamino)ethyl methacrylate; AEMA, 2-aminoethyl methacrylate; DMPA, 2,2-Dimethoxy-2phenylacetophenone. 

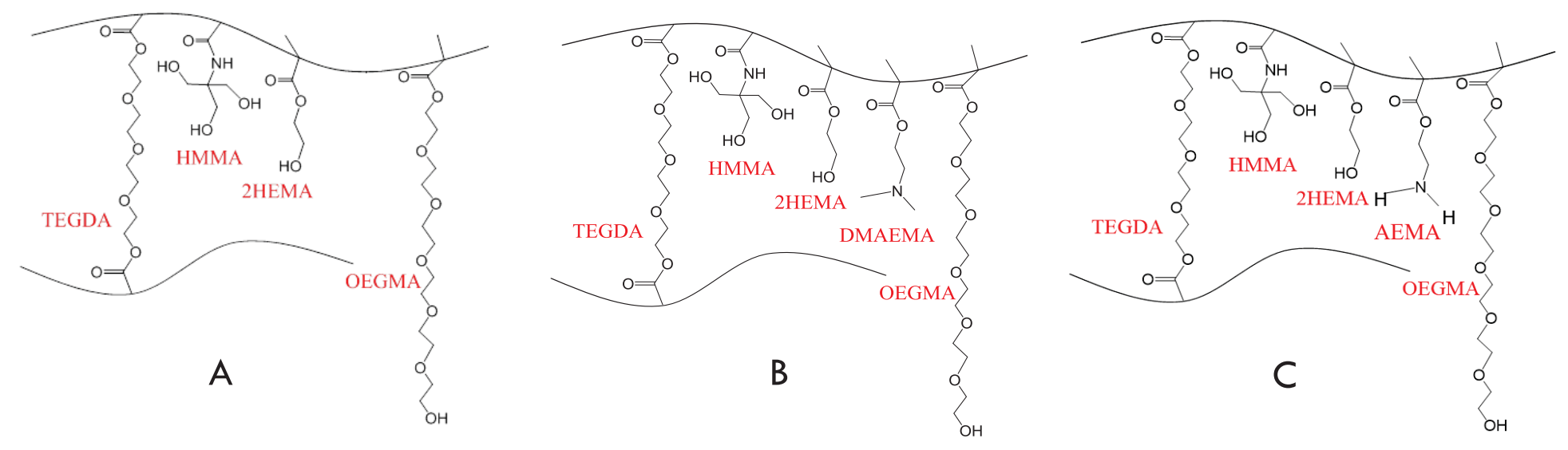

Figure A-1. Schematic illustration of the polymerized hydrogels.

Notes: Control hydrogel (A), DMAEMA modified hydrogel (B), AMEA modified hydrogel (C). 
hermetic lid, 901684.901; Tzero pan, 901683.901). Each sample was equilibrated at -20 ${ }^{\circ} \mathrm{C}$ and run for two consecutive cycles at a heating rate of $10^{\circ} \mathrm{C} / \mathrm{min}$ from $-20^{\circ} \mathrm{C}$ to 200 ${ }^{\circ} \mathrm{C}$. The first heat cycle will assess the hydrogels' properties in the as-molded condition and will erase the thermal history of the hydrogels. The second heat cycle will evaluate the inherent properties of the hydrogels. Three different drying methods were employed: Hydrogel samples were vacuum ( $23 \mathrm{inHg}$ ) dried at room temperature, vacuum dried (23 inHg) at $80^{\circ} \mathrm{C}$, and freeze dried for overnight before the $T_{g}$ measurements.

\section{Hydration and Water States Characterization}

The degree of hydration $(\mathrm{DoH})$ of the hydrogels with and without $5 \mathrm{~mol} \%$ charged modifier DMAEMA /AEMA in DI water at RT $\left(22^{\circ} \mathrm{C}\right)$ was determined by gravimetric analysis. Firstly, following overnight equilibration and the removal of excess surface water with lint-free paper (Kim wipes ${ }^{\mathbb{B}}$ ), the weight of the fully hydrated hydrogels (HG) equilibrated in DI water was measured using an analytical balance $( \pm 0.1$ $\mathrm{mg}$ ). Hydrogel samples were then dried to constant weight using Freeze Dry System (Freezone 4.5, Labconco). The samples were further kept in the $-80^{\circ} \mathrm{C}$ freezer for $12 \mathrm{~h}$ then allowed to dry in the lyophilizer for 2 days at a pressure of $100 \mathrm{mtorr}$ and $-80^{\circ} \mathrm{C}$ and the weight repeatedly measured to constant weight. Equation A-1 was used to calculate the percentage hydration is given below

$$
D O H(\mathrm{wt} \%)=\left(\frac{M_{H G}-M_{D G}}{M_{H G}}\right) 100 \%
$$

Where $M_{H G}$ is the weight of the hydrated hydrogel at equilibration, $M_{D G}$ is the weight of the dry hydrogel, $M_{H G}-M_{D G}$ is the equilibrium water content $(E W C)$ of the hydrogel

The water states in the hydrated hydrogels containing $3 \mathrm{~mol} \%$ TEGDA crosslinker with and without $5 \mathrm{~mol} \%$ DMAEMA were measured and calculated using a differential scanning calorimeter (DSC) (Q2000, TA Instruments) equipped with a lowtemperature cooling module and using, the method previously reported with slight modification [19-21]. Briefly, the hydrated hydrogels in DI water with known DoH were surface wiped, weighed and sealed in individual hermetically sealed aluminum pans (Tzero hermetic lid, 901684.901; Tzero pan, 901683.901). Samples were equilibrated at $40{ }^{\circ} \mathrm{C}$ and then heated up to $30{ }^{\circ} \mathrm{C}$ at a rate of $10{ }^{\circ} \mathrm{C} / \mathrm{min}$ under constant purging of nitrogen at $50 \mathrm{~mL} / \mathrm{min}$. For each measurement, five samples were analyzed. The enthalpy of the endotherm, $\Delta H_{m}$, at $0{ }^{\circ} \mathrm{C}$ was obtained by integration of the endothermic peak using TA Universal Analysis software and normalized to the weight of the hydrated gel with a unit of $\mathrm{J} / \mathrm{g}$ of hydrated gel. The deionized water was used as a reference and the melting enthalpies for freezing free water and freezing bound water were assumed to be the same as that of bulk water $\left(\Delta H_{0}=334 \mathrm{~J} / \mathrm{g}\right)$. Small differences in the enthalpies of the melting ice of different crystals were neglected. Ratio $\Delta H_{\mathrm{m}} / \Delta H_{0}$ represents grams of freezable water per gram of the swollen hydrogel as shown in Equation A-2. 


$$
\frac{\mathrm{W}_{\mathrm{f}}}{\mathrm{M}_{\mathrm{HG}}}(\mathrm{wt} \%)=\frac{\Delta \mathrm{H}_{\mathrm{m}}}{\Delta \mathrm{H}_{0}}
$$

The amount of non-freezing bound water per gram of hydrated gel was taken as the difference between the DoH and the amount of freezing water per gram of hydrated gel using Equation A-3.

$$
\frac{W_{\mathrm{nfb}}}{M_{\mathrm{HG}}}(\mathrm{wt} \%)=D o H-\frac{W_{\mathrm{f}}}{M_{\mathrm{HG}}}
$$

\section{Results and Discussion}

The presence of the hydrophobic but potentially ionizable $3^{\circ}$ amine, DMAEMA, as a co-monomer at $5 \mathrm{~mol} \%$ is expected to alter the mechanical and thermal properties of the poly(HEMA)-based hydrogel. Such change, reflected in the physicochemical properties of the hydrogels, may arise because of the altered structure, potential crosslinking and hydration characteristics of the hydrogel that is co-incident to the chemical modification of the backbone.

\section{Mechanical Properties of Hydrogels}

Figure A-2A. illustrates the LVE region of the hydrogels under oscillatory strain sweep. Figure A-2B shows the storage $G^{\prime}$ and loss $G^{\prime \prime}$ moduli of the hydrogels with and without $5 \mathrm{~mol} \%$ DMAEMA as a function of frequency. There was no difference between both the $\mathrm{G}^{\prime}$ and $\mathrm{G}^{\prime \prime}$ of the two types of hydrogels over the entire frequency range from 0.1 to $100 \mathrm{~Hz}$. The $\mathrm{G}^{\prime}$ was always higher than $\mathrm{G}^{\prime \prime}$ suggesting that the elastic behavior dominates the viscous behavior in these hydrogels, which is the typical characteristic of a hydrogel. Figure A-2B also shows that both the $\mathrm{G}^{\prime}$ and $\mathrm{G}^{\prime \prime}$ increased linearly with increasing frequency, suggesting that the both types of hydrogels were in the rubbery to glassy transition stage and became stiffer with increasing frequency. Figure A-3 shows the stress-strain profiles resulted in the compression measurements of the hydrogels with and without $5 \mathrm{~mol} \%$ DMAEMA. The addition of $5 \mathrm{~mol} \%$ DMAEMA increased the onset stress and onset strain from $5 \pm 1 \mathrm{kPa}$ and $4.3 \pm 0.6 \%$ to $13 \pm \mathrm{kPa}$ and $7.1 \pm 1.1 \%$, respectively (Figure A-3A). More significantly, the addition of $5 \mathrm{~mol} \%$ DMAEMA increased the compression modulus three-fold, from $1.5 \pm$ to $4.8 \pm \mathrm{MPa}$ (Figure A-3B). Incorporation of hydrophobic modifiers may increase the toughness of the hydrogels [464]. The results could be explained by the higher hydrophobicity of the DMAE moiety than the HE moiety acting to reduce the total free water content and hence compressibility. Also, the additional crosslinking caused by the hydrogen bond to DMAE and other components of the hydrogels such as HEMA or/and HMMA may also be a factor in increasing the compressibility (Figure A-3C). In combination, the results shown in Figures A-2A, B and A-3A, B confirm that the addition of $5 \mathrm{~mol} \%$ DMAEMA did not change the viscoelastic shear properties of the hydrogels, but significantly increased the hydrogels' resistance to compressive deformation. 

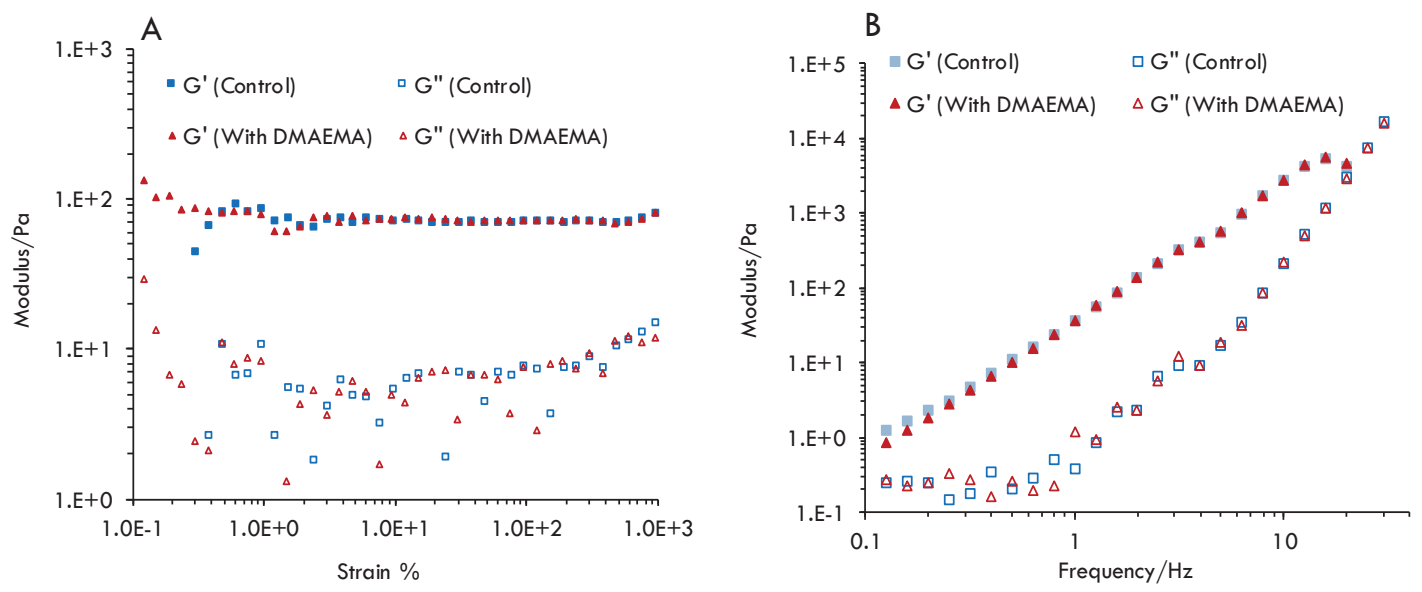

Figure A-2. Shear storage modulus $\left(G^{\prime}\right)$ and shear loss modulus $\left(G^{\prime \prime}\right)$ as a function of strain \% (A) and frequency (B) for the hydrogels.

Notes: Control hydrogels - no DMAEMA; Modified Hydrogels - 5 mol\% DMAEMA. 

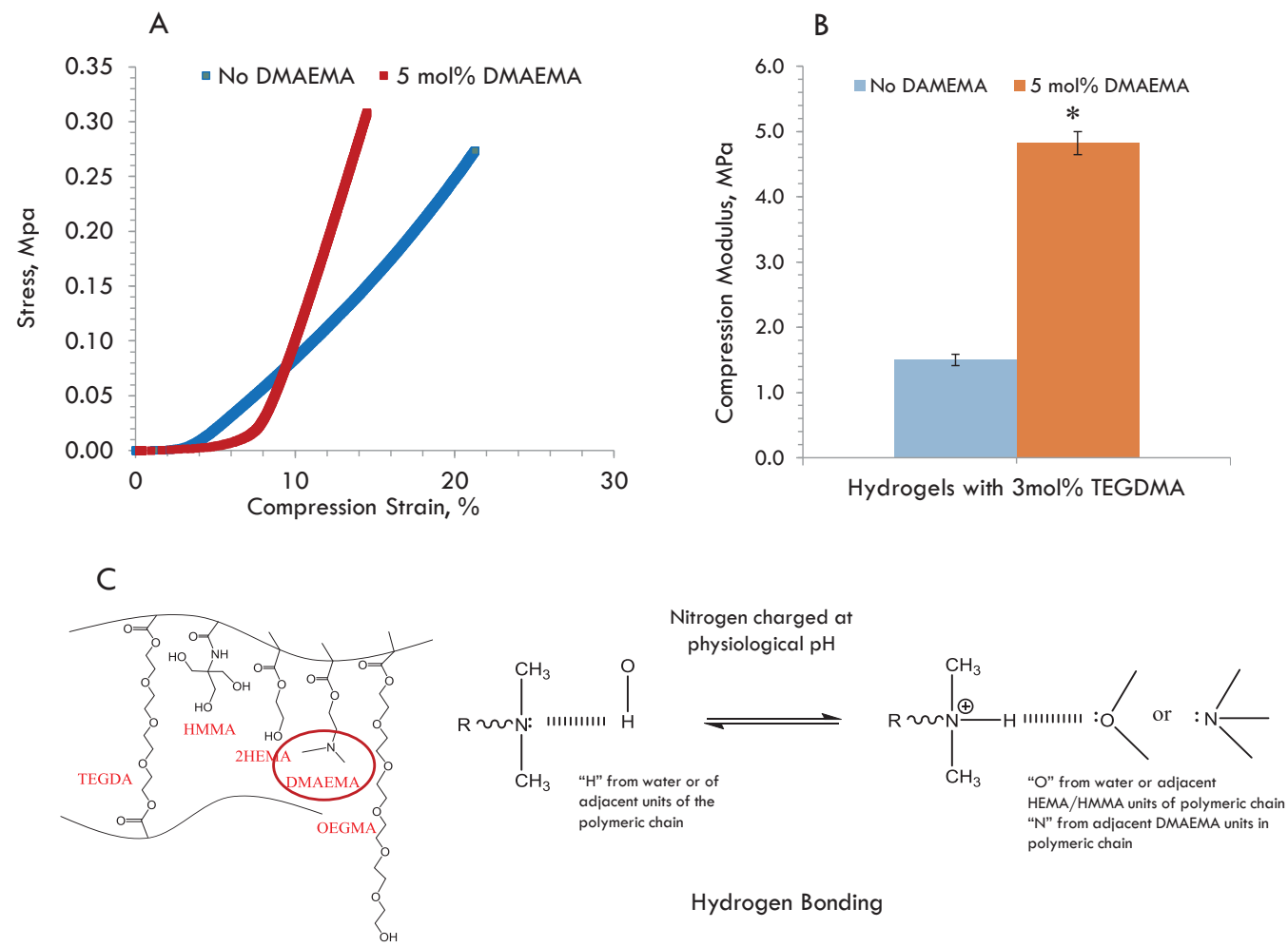

Figure A-3. Stress-strain curve for hydrogels under compression (A); Compression modulus of hydrogels (B); Scheme representing the hydrogen bonding ability of DMAEMA (C).

Notes: Control hydrogels - no DMAEMA; Modified Hydrogels - 5 mol\% DMAEMA Compression modulus data represents mean $\pm \mathrm{SD}, \mathrm{n}=3$. Hydrogel structure reprinted with permission. 


\section{Glass Transition Temperature $\left(T_{g}\right)$ of Hydrogels}

DSC was used to determine the glass transition temperatures $\left(T_{g}\right)$ of the hydrogels with and without $5 \mathrm{~mol} \%$ DMAEMA. The $T_{g}$ values were obtained from the second heat cycle of the hydrogels dried through three different processes: vacuum ( $23 \mathrm{in} \mathrm{Hg}$ ) at room temperature, vacuum (23 in $\mathrm{Hg}$ ) at $80^{\circ} \mathrm{C}$, and freeze drying are shown in Table A-2. The drying condition had a considerable influence on the measured $T_{g}$. For the hydrogels without $5 \mathrm{~mol} \%$ DMAEMA, vacuum drying at $80{ }^{\circ} \mathrm{C}$ caused the $T_{g}$ to shift to a higher temperature than the vacuum drying at room temperature, and the freeze drying shifted the $T_{g}$ to an even higher temperature than the vacuum drying at $80{ }^{\circ} \mathrm{C}$. For the hydrogels with $5 \mathrm{~mol} \%$ DMAEMA, the vacuum drying at room temperature and the freeze drying produced a similar $T_{g}$, while the vacuum drying at $80{ }^{\circ} \mathrm{C}$ caused the $T_{g}$ to shift to a higher temperature than the other two drying processes. The reason might be that the different drying processes resulted in the hydrogels having different amounts of residual water which acted as a plasticizer to decrease the $T_{g}$. The vacuum drying at $80{ }^{\circ} \mathrm{C}$ and freeze drying made the hydrogels drier (containing less water) than the vacuum drying at room temperature. Depending on the chemical structures of the hydrogels (with or without DMAEMA), the vacuum drying at $80{ }^{\circ} \mathrm{C}$ could cause the hydrogels to contain more or less water than freeze drying. However, irrespective of the drying condition, in all three drying processes it was observed the addition of $5 \mathrm{~mol} \%$ of DMAEMA increased the $T_{g}$ of the polymer.

It is well known that the value of $T_{g}$ depends on the mobility of the polymer chain, the more immobile the chain, the higher the value of $T_{g}$ [465]. In this case, the primary factors that may have played a role in increasing the $T_{g}$ of the polymer after incorporation of DMAEMA are: water content and cross-linking. It is reported in the literature that water acts as a plasticizer by reducing the interaction forces between the polymer chains-i.e., the hydrogen bonds and improve flexibility thereby lower $T_{g}$ [466]. As DMAEMA is hydrophobic, replacement of 5 mol\% HEMA with DMAEMA might have imparted hydrophobicity to the hydrogels and may have lowered the water content of the hydrogels and has increased $T_{g}$. Further, as increased crosslinking between the chains restricts the polymer mobility [467], the physical crosslinks formed by the hydrogen bonding between the DMAEMA and poly(HEMA) polymeric network might be another factor that caused the shift to higher $T_{g}$. To confirm these assumptions hydration studies and swelling studies were conducted with both modified and control hydrogels.

\section{Hydration and Water States within Hydrogels}

The physicochemical, transport and sorption properties of hydrogels are re expected to be strongly influenced by the amount of water and the distribution of this water among the many possible states within the hydrogel [468]. Therefore to study the

effect of the modification on hydrogel properties, hydration studies were conducted. The DoH of the control and DMAEMA modified hydrogels obtained in water is presented Table A-3. The presence of $5 \mathrm{~mol} \%$ DMAEMA dramatically alters the hydration 
Table A-2. The glass transition temperatures $\left(T_{g}\right)$ from the second heat cycle for the hydrogels with and without 5 mol\% DMAEMA under different drying processes.

\begin{tabular}{|c|c|c|c|c|c|c|}
\hline \multirow{2}{*}{$\begin{array}{l}\text { Hydrogel/ } \\
T_{g}\left({ }^{\circ} \mathrm{C}\right)\end{array}$} & \multicolumn{2}{|c|}{$\begin{array}{c}\text { Vacuum dry at room } \\
\text { temperature }\end{array}$} & \multicolumn{2}{|c|}{ Vacuum dry at $80^{\circ} \mathrm{C}$} & \multicolumn{2}{|c|}{ Freeze dry } \\
\hline & Onset & Midpoint & Onset & Midpoint & Onset & Midpoint \\
\hline Control & 47.14 & 67.78 & 69.57 & 75.60 & 80.02 & 89.73 \\
\hline DMAEMA modified & 79.47 & 99.99 & 102.80 & 111.06 & 85.87 & 98.19 \\
\hline
\end{tabular}


Table A-3. The gravimetrically determined degree of hydration ( $\mathrm{DoH} \%)$ of the p(HEMA)-based hydrogels with and without DMAEMA.

\begin{tabular}{lccc}
\hline Hydrogels & $\begin{array}{c}\text { Dry gel, } \\
\text { mg }\end{array}$ & $\begin{array}{c}\text { Hydrated gel, } \\
\text { mg }\end{array}$ & $\begin{array}{c}\text { Degree of hydration, } \\
\mathbf{w t} \%\end{array}$ \\
\hline Control & $22.9 \pm 0.6$ & $41.6 \pm 0.6$ & $43.7 \pm 1.3$ \\
With 5 mol\% DMAEMA & $22.5 \pm 0.8$ & $34.4 \pm 0.5$ & $31.7 \pm 1.2$ \\
\hline
\end{tabular}


characteristics of the $\mathrm{p}$ (HEMA)-based hydrogel, reducing its $\mathrm{DoH}$ from $43.7 \pm 1.28$ to $31.7 \pm 1.17$. The hydrophobicity of DMAEMA significantly reduced the water intake into the hydrogels, and the modified hydrogels were less swollen compared to the control. To more deeply investigate the distribution of water states within these hydrogels, the 3 mol\% TEGDA crosslinker formulation possessing 0 mol\% or 5 mol\% DMAEMA was studied by DSC following equilibration in DI water or HEPES buffer. Water present in hydrated hydrogels is known to be present in three conditions, (i) freezable free water $\left(W_{f f}\right)$, (ii) freezable bound water $\left(W_{f b}\right)$ (freezing point depressed), and (iii) and nonfreezable bound water $\left(W_{n f b}\right)$. The freezable free water $W_{f f}$ does not participate in any interaction with the polymer chains while the freezable bound $W_{f b}$ water slightly interacts with the polymer chains. The sum of these two states of water is collectively named freezable water $\left(W_{f}\right)$ which exhibits a phase transition at a temperature of $0{ }^{\circ} \mathrm{C}$. On the other hand, the non-freezable bound water $W_{n f b}$ interacts strongly with the polymer chains through hydrogen bonding and does not show any phase transition at $0{ }^{\circ} \mathrm{C}$. The sum of the freezable water $\left(W_{f}\right)$ and non-freezable bound water $W_{n f b}$ normalized by the weight of the hydrated hydrogel at equilibration $(M H G)$ equals to the degree of hydration $\mathrm{DoH}$ as shown below in Equation A-4. Thus, the DoH determined by DSC may be directly compared with the $\mathrm{DoH}$ obtained by gravimetry and is expected to be in close agreement.

$$
D o H(\mathrm{wt} \%)=\frac{W_{\mathrm{f}}+W_{\mathrm{nfb}}}{M_{\mathrm{HG}}}=\frac{\left[W_{\mathrm{ff}}+W_{\mathrm{fb}}\right]+W_{\mathrm{nfb}}}{M_{\mathrm{HG}}}
$$

Figure A-4 shows that warming the frozen hydrated hydrogels containing hydrogels with and without $5 \mathrm{~mol} \%$ DMAEMA resulted in a broad ice-melting endodermic peak at around $0{ }^{\circ} \mathrm{C}$ correlated to the freezable free water in each hydrogel. The endothermic peak is smaller for hydrogels containing 5 mol\% DMAEMA suggesting less overall hydration. The broad peak at around $0{ }^{\circ} \mathrm{C}$, representing freezable free water within the hydrogels, was split into two not so clearly resolved peaks which might be due to different ice structures of the freezable free water within the hydrogels [469]. Figure A-4 also shows that the $0{ }^{\circ} \mathrm{C}$ peak areas of the hydrogels with DMAEMA were significantly lower than those of the hydrogels without DMAEMA. Besides the peak at around $0{ }^{\circ} \mathrm{C}$, the hydrogels without DMAEMA showed another relatively small broad peak at around $-6{ }^{\circ} \mathrm{C}$. This little broad peak could be due to the water that was loosely bound to the hydrogel polymer chains and could be frozen but at a temperature lower than $0{ }^{\circ} \mathrm{C}$ (depression of freezing point) [469-471]. Further observation showed that the small broad peak at around $-6{ }^{\circ} \mathrm{C}$ was stronger in the hydrogels without DMAMEA that were equilibrated in HEPES buffer than the same hydrogel that was equilibrated in DI water, indicating that the buffer salts enhanced water to be bound to the polymer chains, consistent with the literature report [472]. As the areas of the endothermic peaks at around $0{ }^{\circ} \mathrm{C}$ and around $-6{ }^{\circ} \mathrm{C}$ correlate to the freezable free water content and freezable bound water content, respectively, we further measured the peak areas and calculated the freezable water content which equals the sum of freezable free water content and freezable bound water content. By using Equation A-4, the non-freezable bound water content was calculated from the $\mathrm{DoH}$ obtained from the gravimetric measurements at 25 ${ }^{\circ} \mathrm{C}$ and the freezable water content obtained from the DSC results of the hydrogels and listed the results in Table A-4. 




Figure A-4. Endothermic curves of swollen hydrogels synthesized with and without 5 mol\% DMAEMA. 
Table A-4. Distribution of water content in the hydrogels possessing 0 or 5 mol \% DMAEMA and equilibrated in DI water.

\begin{tabular}{|c|c|c|c|c|c|c|}
\hline \multirow[t]{2}{*}{ Hydrogels } & \multicolumn{2}{|c|}{$\begin{array}{c}\text { Degree of hydration } \mathrm{DoH} \\
(\mathrm{wt} \%)\end{array}$} & \multicolumn{2}{|c|}{$\begin{array}{c}\text { Freezable water } \frac{W_{\mathrm{f}}}{M_{\mathrm{HG}}} \\
(\mathrm{wt} \%)\end{array}$} & \multicolumn{2}{|c|}{$\begin{array}{c}\text { Non-freezable bound water } \frac{W_{\text {nfb }}}{M_{\mathrm{HG}}} \\
(\mathrm{wt} \%)\end{array}$} \\
\hline & $\mathrm{DI} \mathrm{H}_{2} \mathrm{O}$ & HEPES & $\mathrm{DI} \mathrm{H}_{2} \mathrm{O}$ & HEPES & $\mathrm{DI} \mathrm{H}_{2} \mathrm{O}$ & HEPES \\
\hline Control & $43.7 \pm 1.3$ & $43.4 \pm 0.5$ & $18.7 \pm 1.4$ & $18.6 \pm 0.3$ & $25.0 \pm 0.9$ & $24.8 \pm 0.6$ \\
\hline DMAEMA modified & $31.7 \pm 1.2$ & $29.7 \pm 1.1$ & $5.5 \pm 0.6$ & $3.8 \pm 0.5$ & $26.2 \pm 0.9$ & $25.9 \pm 0.9$ \\
\hline
\end{tabular}


Table A-4 shows that a small amount ( $5 \mathrm{~mol} \%$ ) of the hydrophobic DMAEMA can dramatically alter the hydration characteristics of cross-linked poly(HEMA). From the results it can be observed that the major part of the equilibrium water content in the hydrogels with and without 5 mol\% DMAEMA is in non-freezable bound water state and the addition of $5 \mathrm{~mol} \%$ DMAEMA did not have much influence on the non-freezable bound water amount. However, the addition of $5 \mathrm{~mol} \%$ DMAEMA significantly decreased the amount of freezable water three-fold (18.7 \pm 1.4 vs. $5.5 \pm 0.6$ in DI water; $18.6 \pm 0.3$ vs. $3.8 \pm 0.5$ in HEPES buffer) and thus the total hydration (43.7 \pm 1.3 vs. 31.7 \pm 1.2 in DI water; $43.4 \pm 0.5$ vs. $29.7 \pm 1.1$ buffer), and also disturbed the loose binding between the water and the polymer chains causing the disappearance of the small peak at around $-6{ }^{\circ} \mathrm{C}$ in Figure A-4. The reason might be because the non-freezable bound water is derived from water that is strongly bound to the polar hydroxyl groups of the poly(HEMA) repeat unit via hydrogen bonds [473] and since poly(HEMA) was the majority component with a similar amount in both types of hydrogels one may expect the non-freezable water content to be about the same. The freezable water is derived from the free or slightly bound water that resides within the nano-dimensioned pores of the hydrogels [474, 475]. The addition of $5 \mathrm{~mol} \%$ DMAEMA could make the pores effectively smaller because of the physical crosslinking by hydrogen bonding with the poly(HEMA) and also the free water in the pores might have been repelled as the DMAEMA moiety $\left[\delta_{\text {DMAEMA }}=27.92(\mathrm{cal} \cdot \mathrm{cm}-3) 1 / 2\right]$ is more hydrophobic than the HEMA moiety $\left[\delta_{\text {HEMA }}=31.2(\mathrm{cal} \cdot \mathrm{cm}-3) 1 / 2\right]$ [476-478], as reflected in their respective solubility parameters, $d$, calculated from group contributions. The presence of the HEPES buffer did not affect the non-freezable bound water content in both the hydrogels with and without $5 \mathrm{~mol} \%$ DMAEMA, and as well as the $\mathrm{DoH}$ and the freezable water contents in the hydrogels without DMAEMA. However, the presence of the HEPES buffer slightly decreased the $\mathrm{DoH}$ and the freezable water contents in the hydrogels with $5 \mathrm{~mol} \%$ DMAEMA, probably due to the salting out effect as the DMAEMA is slightly positively charged at $\mathrm{pH}$ 7.4. The hydrogels without DMAEMA in both water and HEPES buffer had similar freezable water content but higher freezable bound water content in HEPES buffer (Figure A-4), suggested that the salts in the buffer redistributed the freezable water within the hydrogels. Further investigation is needed to better understand the exact mechanisms for the observed redistribution of water content within the DMAEMA hydrogels. However, there is an indication that this may arise from the unique mesostructure adopted by the amphiphilic nature of the hydrogel [479]. It is noteworthy that the $\mathrm{DoH}$ values at $25^{\circ} \mathrm{C}$ in Table A-4 are similar to the corresponding gravimetrically determined $\mathrm{DoH}$ values at $37^{\circ} \mathrm{C}(43.7 \pm 1.3$ and $31.7 \pm 1.2 \%$ vs. $42 \pm 1.4$ and $34 \pm 1.2 \%$ in DI water; and $43.4 \pm 0.5$ and $29.7 \pm 1.1 \%$ vs. $41.3 \pm 0.8$ and $33.1 \pm 1.0$ in HEPES), suggesting that changing temperature from $37{ }^{\circ} \mathrm{C}$ to $25^{\circ} \mathrm{C}$ did not appreciably affect the degree of hydration of the hydrogels. Reduced freezable water of gels containing DMAEMA are due to hydrophobicity and physical cross-linking [480, 481] within the hydrogel via hydrogen bonding resulting in a tight network. The results were in agreement with the earlier assumption that the modification of hydrogels with hydrophobic cationic modifier DMAEMA altered the degree of hydration and water states of the hydrogels thereby affected the physicochemical properties of the hydrogels. 
To understand the effect of a hydrophilic cationic modifier, 5 mol\% AEMA was added instead of DMAEMA, and the hydration studies were repeated with AEMA modified hydrogels. Comparison of hydration characteristics of all the three hydrogels in DI water is given in Table A-5. The addition of $5 \mathrm{~mol} \%$ AEMA to the control hydrogels did not resulted in any change in the equilibrium water content of the hydrogels but significantly decreased the bound water content in the hydrogels from $25.0 \pm 0.93$ to 16.5 \pm 0.27 , and increased the freezable water content from $18.7 \pm 1.43$ to $27.8 \pm 0.95$. Increased hydrophilicity of the AEMA hydrogels may have led to improved $\mathrm{DoH}$ of the gels compared to the DMAEMA hydrogels (44.3 vs. 31.7), however, the reason for lower bound water could be attributed to the hydrogen bonding of primary hydrogen atoms attached to the amine of the AMEA moiety with HEMA/HMMA units, thereby limiting the hydrogen bonding chances of water with HEMA/HMMA units in the polymer chain. This interference might have resulted in lowering the bound water content of the hydrogels after addition of AEMA (Figure A-5). This interpretation of the results needs further investigation.

\section{Conclusions}

The addition of cationogenic DMAEMA at low mol\% (5 mol\%) has significantly altered the mechanical, thermal and hydration properties of the hydrogels. The incorporation of $5 \mathrm{~mol} \%$ DMAEMA into HEMA-based hydrogels did not alter the shearing modulus. However, this changed has resulted in an increase in the compression modulus and $T_{g}$ of the hydrogels, owing to the hydrophobicity and physical crosslinking of the DMAEMA. The degree of hydration and the distribution of water within the hydrogels varied significantly depending on the hydrophobicity/hydrophilicity \& hydrogen bond forming ability of the cationic modifier used in the gels thereby affecting the hydrogel properties. Freeze drying produced the most consistent drying condition.

\section{Future Work}

Some of the near future work on this project include - i) continuing evaluation of the AEMA modified hydrogels for the physicochemical properties for further understanding the influence of cationogenic modifiers with hydrophilic and hydrophobic characteristics; ii) evaluating the effect of inclusion of both AEMA and DMAEMA as cationic modifiers in the hydrogels; and iii) optimizing the hydrogels for loading and release of biomolecules for drug delivery applications. 
Table A-5. Distribution of water content of hydrogel possessing 0 or $5 \mathrm{~mol} \%$ DMAEMA or AEMA and equilibrated in DI water.

\begin{tabular}{lccc}
\hline Hydrogels & $\boldsymbol{D o H}, \mathbf{w t} \%$ & $\boldsymbol{W}_{\boldsymbol{f}, \mathbf{w t} \%}$ & $\boldsymbol{W}_{\boldsymbol{n} \boldsymbol{b} \boldsymbol{f}, \mathbf{w t} \%}$ \\
\hline Control Hydrogel & $43.7 \pm 1.28$ & $18.7 \pm 1.43$ & $25.0 \pm 0.93$ \\
5 mol\% DMAEMA & $31.7 \pm 1.17$ & $5.5 \pm 0.64$ & $26.2 \pm 0.86$ \\
5 mol\% AEMA & $44.3 \pm 1.15$ & $27.8 \pm 0.95$ & $16.5 \pm 0.27$ \\
\hline
\end{tabular}






Hydrogel with AEMA



Hydrogen Bonding

Figure A-5. Scheme representing the possible hydrogen bonding between AEMA units with adjacent HMMA or HEMA units.

Notes: The hydrogens on AEMA might have involved in H-bonding with HEMA or HMMA units on the hydrogel network and thereby limiting the bonding of water to gels; thereby minimizing the bound water content. Though the primary hydrogens of amine (AEMA) could also involve in hydrogen bonding with water molecules, but the bonding of these hydrogen's with HEMA/HMMA might be more predominant than bonding to water. Hydrogel structure modified with permission. 


\section{VITA}

Mr. Dileep Reddy Janagam was born in Yedapally village, Nizamabad District, Telangana, India in 1986. He received his Bachelor of Pharmacy degree from University College of Pharmaceutical Sciences, Kakatiya University in 2008. After receiving his BS he worked as a Junior Analyst at Thomson Reuters, Hyderabad, India. In the fall of 2009, he enrolled in Eastern New Mexico University, Portales, NM as a master student and received his Master of Science Degree in Chemistry in July 2011. Dileep enrolled in the Ph.D. program in Department of Pharmaceutical Sciences at The University of Tennessee Health Science Center (UTHSC), Memphis, TN in August 2011 and became a member of Dr. Tao Lowe's research group. During his tenure at UTHSC, his research work has been directed to the development of biodegradable polymeric biomaterials for drug delivery. During his time at UTHSC, he has presented his work in various national and regional conferences including AAPS, SFB, BMES, and ACS.

He is a member of Rho Chi, The American Association of Pharmaceutical Sciences (AAPS) and Society for Biomaterials (SFB). He actively served the AAPS organization as a student representative for the Physical Pharmacy and Biopharmaceutics Section, and Nanotechnology Focus Group. He also served as a Chair-elect and Chair for the AAPS-UT Student Chapter for the academic years 2014-15, and 2015-16, respectively. 